\title{
Mass Spectrometry-based Analysis to Investigate the Physiochemical and Proteomic Properties of Viral Sensitizers \\ by
}

Andrew Mackendrick Macklin

A thesis submitted to the Faculty of Graduate and Postdoctoral Affairs in partial fulfillment of the requirements for the degree of

Master of Science

in

Chemistry

Carleton University

Ottawa, Ontario

C Copyright 2015, Andrew Mackendrick Macklin 


\begin{abstract}
Attenuated viruses hold great potential in viral-based therapies including vaccine production and oncolytic virotherapy $(\mathrm{OVt})$. Both applications are hindered by poor infection propagation as a result of genetic heterogeneity amongst target cells in regard to antiviral response functionality. Our collaborative research group has developed a novel solution to address this problem: first-in-class small molecules termed viral sensitizers (VSes) that potentiate viral infection in resistant cells. Liquid chromatography mass spectrometry (LC-MS) methods that made use of various scanning modes were developed to characterize the poor physiochemical properties of the first lead compound (VSe1). Furthermore, the LC-MS methods were applied in the screening of a large VSe library and performing quality control (QC) experiments. Subsequently, new lead compounds were identified with improved plasma stability and retained activity. In vivo pharmacokinetic (PK) and metabolomic studies using the new lead VSes revealed that glutathione stability and tumour penetrance are important considerations for future screening of VSes. MS-based and gel-based proteomic experiments made use of target identification techniques to elucidate the VSe mechanism of action (MOA). GSTp1 was studied as a potential target but multiple evidences supported that VSes act as noncovalent inhibitors. Future studies will further address the unknown MOA and in vivo behavior to further narrow the list of lead VSes to application-specific compounds.
\end{abstract}




\section{Acknowledgements}

I would like to whole-heartedly thank my supervisor, and mentor, Dr. Jeff Smith for his guidance and support over the last two years. His unexpected email in December 2012 asking me to start a Masters a few weeks later has turned out to be life-altering, for the better. His confidence in taking me under his wing has translated to my own self-belief as a scientist. Dr. Jean-Simon Diallo and Dr. Chris Boddy have been great supervisors throughout the VSe collaboration as demonstrated by their hungry work ethic. Furthermore, it has been a pleasure to work closely with Ramya Krishnan and Mark Dornan. I can proudly state we made a great team working on a really cool and promising project. I would also like to thank Dr. Bill Willmore for his expertise and generously allowing me to use his cell culture and proteomics equipment.

I would like to appreciatively thank all past and present Smith lab members for making it an enjoyable workplace. In particular, I am grateful for Karl "Kerrigan Jones" Wasslen initially showing me the ropes of MS and his ability to make every day filled with unexpected and interesting conversation. Secondly, thank you to Carlos Canez for being my sing-along partnerin-crime and my crutch for whenever I needed to rant.

I would like to thank all my friends for making my seven years at Carleton an absolutely unforgettable experience. To my girlfriend Maria, I look forward to returning the favours you have given me throughout this degree: understanding, encouragement and sleep to make up for all the late-night practice presentations over Skype. Couples who science together, stay together! Lastly, I'd like to thank my family: Steve, Heidi and my sister Jillian. They have been my number one fans since Day 1 and I cannot express how much your support has shaped who I am today. Most importantly, they have constantly driven me to do the best I can in all of my efforts, including this degree. Thank you for your unconditional love and it is to them that I dedicate this work. 


\section{List of Abbreviations}

\begin{tabular}{|c|c|}
\hline $\mathrm{ABC}$ & Ammonium bicarbonate \\
\hline ABPP & Activity-based protein profiling \\
\hline $\mathrm{AC}$ & Alternating current \\
\hline $\mathrm{ACN}$ & Acetonitrile \\
\hline AUP & Area-under-the-peak \\
\hline $\mathrm{BCA}$ & Bicinchoninic acid \\
\hline CEM & Channel electron multiplier \\
\hline CID & Collision induced dissociation \\
\hline $\mathrm{DC}$ & Direct current \\
\hline DIPCDI & N-N-Diisopropylcarbodiimide \\
\hline DIPEA & Diisopropylethylamine \\
\hline DMEM & Dulbecco's minimum essential medium \\
\hline DMF & Dimethylformamide \\
\hline DMSO & Dimethylsulfoxide \\
\hline DTT & Dithiothreitol \\
\hline ESI & Electrospray ionization \\
\hline FAw & Formic acid in water \\
\hline FBS & Fetal bovine serum \\
\hline GSTp1 & Glutathione s-transferase ( $\pi$ isozyme) \\
\hline HOBT & 1-Hydroxybenzotriazole \\
\hline HPLC & High performance liquid chromatography \\
\hline ID & Inner diameter, identity \\
\hline IDA & Independent data acquisition \\
\hline IEF & Isoelectric focussing \\
\hline IFN & Interferon \\
\hline IP & Intraperitoneal \\
\hline IPG & Immobilized $\mathrm{pH}$ gradient \\
\hline ISG15 & IFN-stimulated protein of $15 \mathrm{kDa}$ \\
\hline IT & Intratumoural \\
\hline LC-MS & Liquid chromatography mass spectrometry \\
\hline LOB & Limit of blank \\
\hline LOD & Limit of detection \\
\hline LOQ & Limit of quantitation \\
\hline $\mathrm{m} / \mathrm{z}$ & Mass to charge \\
\hline MALDI & Matrix assisted laser desorption ionization \\
\hline MASCOT & Protein identification database \\
\hline $\mathrm{MeOH}$ & methanol \\
\hline MOA & Mechanism of action \\
\hline MRM & Multiple reaction monitoring \\
\hline $\mathrm{MS} / \mathrm{MS}$ & Tandem mass spectrometry \\
\hline Mx1 & Myxovirus resistance-1 \\
\hline NK & Natural killer \\
\hline
\end{tabular}




$\begin{array}{ll}\text { NL } & \text { Neutral loss } \\ \text { OHRI } & \text { Ottawa Hospital Research Institute } \\ \text { OVs } & \text { Oncolytic viruses } \\ \text { OTt } & \text { Oncolytic virotherapy } \\ \text { PBS } & \text { Phosphate-buffered saline } \\ \text { pI } & \text { Isoelectric point } \\ \text { PK } & \text { Pharmacokinetics } \\ \text { PKR } & \text { Protein kinase R } \\ \text { PR } & \text { Peroxiredoxin } \\ \text { QC } & \text { Quality control } \\ \text { Q-TOF } & \text { Hybrid quadrupole time-of-flight } \\ \text { R\&D } & \text { Research and development } \\ \text { RF } & \text { Radio frequency } \\ \text { RFP } & \text { Red fluorescent protein } \\ \text { RNaseL } & \text { RibonucleaseL } \\ \text { RP } & \text { Reversed-phase } \\ \text { RT } & \text { Retention time } \\ \text { SAX } & \text { Strong anion exchange } \\ \text { SCX } & \text { Strong cation exchange } \\ \text { SDS-PAGE } & \text { Sodium dodecyl sulfate polyarcrylamide gel electrophoresis } \\ \text { Th } & \text { Thomson } \\ \text { TIC } & \text { Total ion chromatogram } \\ \text { TNBS } & \text { Trinitrobenzene sulfonic acid } \\ \text { TOF } & \text { Time-of-flight } \\ \text { UPLC } & \text { Ultra performance liquid chromatography } \\ \text { VSe } & \text { Viral sensitizer } \\ \text { VSV } & \text { Vesicular stomatitis virus } \\ \text { XIC } & \text { Extracted ion chromatogram } \\ & \end{array}$




\section{Table of Contents}

Abstract................................................................................................................................... 1

Acknowledgements ........................................................................................................................ 2

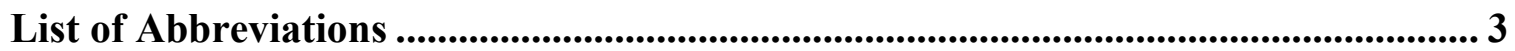

Table of Contents .......................................................................................................................... 5

List of Tables ................................................................................................................................. 7

List of Figures.................................................................................................................................. 8

1 Chapter: Introductory Topics ...................................................................................... 15

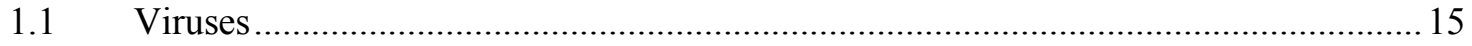

$1.2 \quad$ Vaccine Production ......................................................................................... 19

$1.3 \quad$ Cancer and Oncolytic Virotherapy …………………..........................................2.

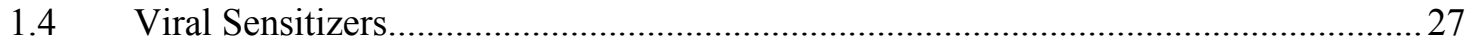

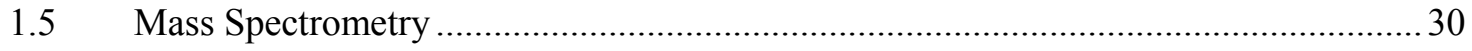

2 Chapter: Sample Preparation and MS Methods ....................................................... 43

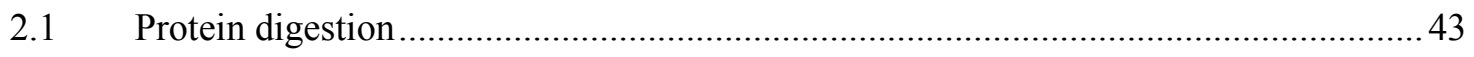

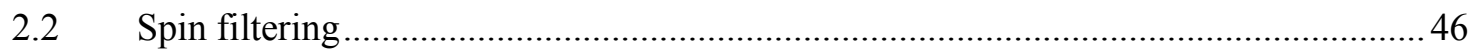

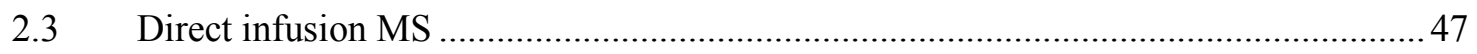

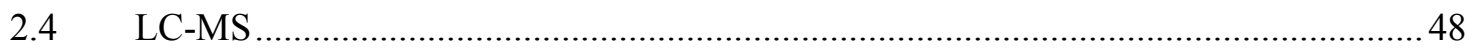

3 Chapter: VSe Quality Control Analysis ..................................................................... 55

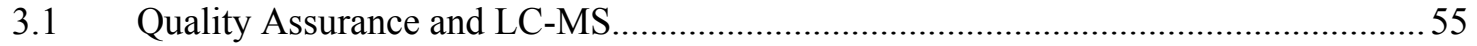

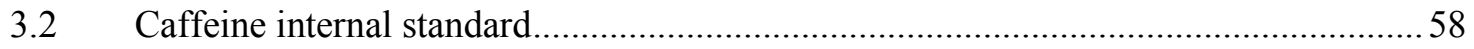

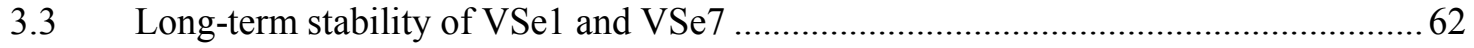

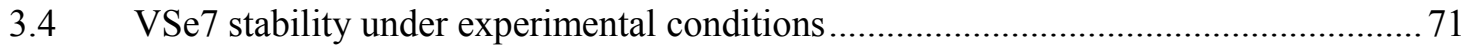

3.5 Absolute quantitation of VSe7 drug preparation and method validation .....................72 


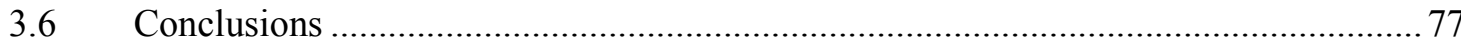

4 Chapter: In vitro and in vivo VSe stability............................................................. 79

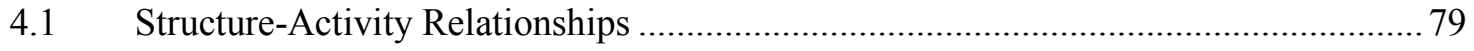

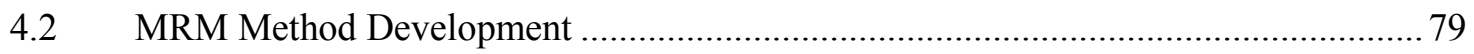

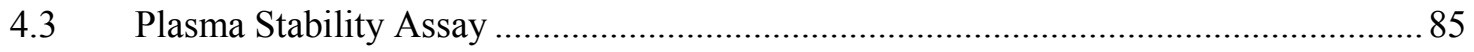

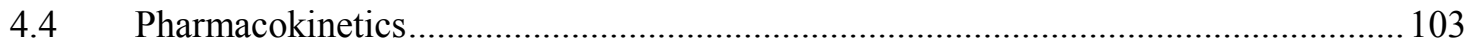

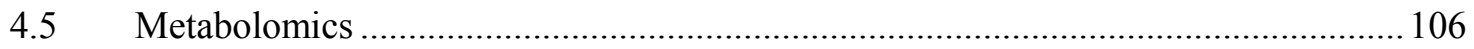

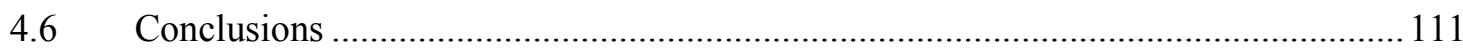

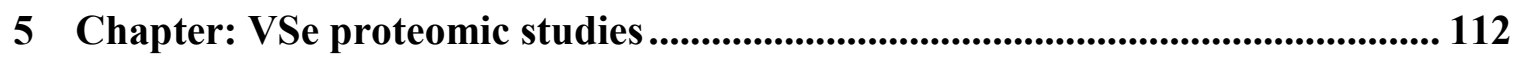

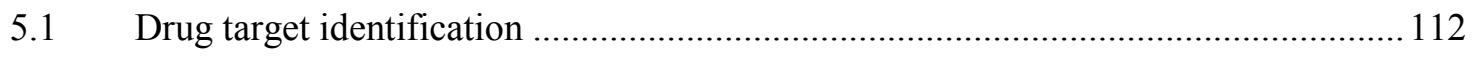

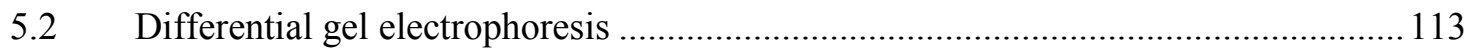

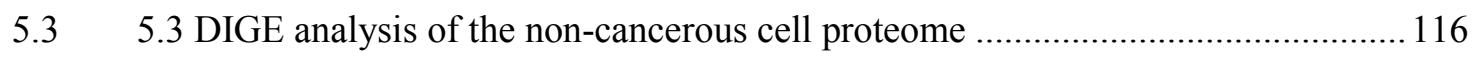

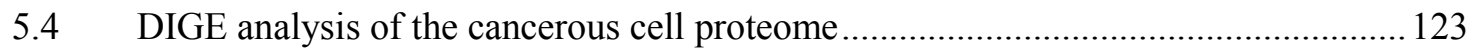

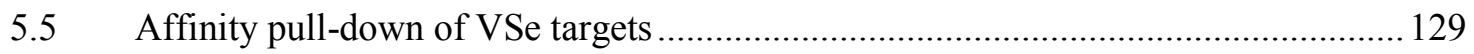

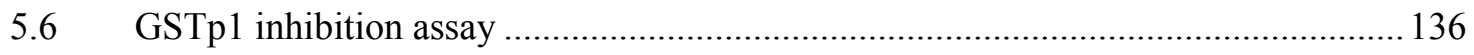

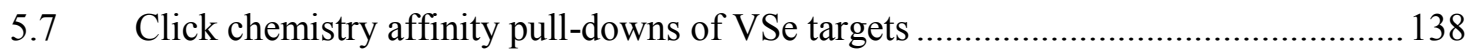

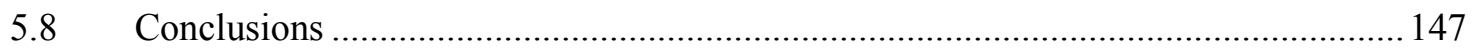

6 Chapter: Concluding Remarks................................................................................. 148

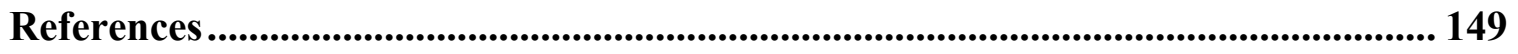




\section{List of Tables}

Table 1.1: Proton affinities of applicable compounds and solvents [54] ....................... 42

Table 3.1: The absolute quantitation of various VSe7 preparations ............................ 73

Table 4.1: VSe MRM parameters used to monitor drug stability ................................. 84

Table 4.2: Diversifying the VSe1 ring scaffold in search of improved stability ............ 89

Table 4.3: Exploring the importance of each MD01145 functional group ................... 91

Table 4.4: Diversifying the R-group extending from the MD01145 amide .................. 93

Table 4.5: Ranking the VSe analogues in order of highest (green) to lowest (red) plasma

stability. 96

Table 4.6: Altering the proximity and electronegativity of EW R-groups affects stability 101

Table 4.7: Plasma stability is dependent on steric hindrance surrounding the hydrolysable amide 101

Table 4.8: R-group unsaturation leads to decreased plasma stability 102

Table 5.1: 786-0 cell counts of negative control and MD01145-treated cultures.......... 124

Table 5.2: Possible peptide ions modified by VSe1 and GSH that went undetected .... 137 


\section{List of Figures}

Figure 1.1: IFN response in neighbouring cells prepares them for viral infection. Adapted from [6] 18

Figure 1.2: Wild-type VSV M-protein blocks the transport of IFN to the cytoplasm (A). VSV $\Delta 51$ selectively infects cancer cells based on this principle (B \& C). 27

Figure 1.3: VSe1 was the most active compound by enhancing VSV $\Delta 51-R F P$ replication in OV-resistant 4T1 cells 34-fold [34] 29

Figure 1.4: VSe1 selectively enhances OV replication in multiple resistant cancerous cell lines [34] 29

Figure 1.5: The essential components of a mass spectrometer 31

Figure 1.6: General schematic depicting electrospray ionization (ESI) [43].................. 32

Figure 1.7: Layout of the QStar XL front end [44]........................................................ 33

Figure 1.8: The alternating RF electric field on the quadrupole electrodes control ion motion and transmission 34

Figure 1.9: The various scanning modes enabled by tandem MS [46] ........................... 35

Figure 1.10: Schematic diagram of a QStar XL QqTOF mass spectrometer [44]........... 36

Figure 1.11: An ion mirror in a TOF mass analyzing corrects ion trajectories [48]........ 37

Figure 1.12: The magnetic fields imposed upon an ion in a quadrupole LIT [44] .......... 37

Figure 1.13: Ion impingement at an $\mathrm{MCP}$ detector leads electron emission and electrical

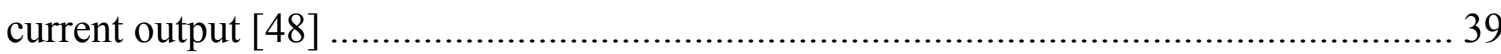

Figure 1.14: The CID fragmentation patterns of peptides. Adapted from [53] ............... 41 Figure 2.1: The reduction and alkylation of disulfide bonds to prepare proteins for tryptic digestion 44 
Figure 2.2: General procedure for the in-gel tryptic digestion of proteins 46

Figure 2.3: Spin filtering to isolate VSes from complex biological samples. Adapted from $[58]$

Figure 2.4: Schematic diagram of the LC-MS switching valve set to the load (A) and inject (B) positions....... 50

Figure 2.5: 30 min proteomics gradient for the analysis of peptides on the QStar XL ... 52 Figure 2.6: 30 min sensitizer gradient for the analysis of VSe stocks and metabolites on the QStar XL 53

Figure 2.7: VSe Gradient A for the analysis of VSe stocks and metabolites on the 4000 Qtrap MS 53

Figure 2.8: VSe Gradient B for the analysis of VSe stocks and metabolites on the 4000 Qtrap MS 54

Figure 3.1: Direct infusion accurate mass scan of caffeine. 59

Figure 3.2: LC-MS of a $250 \mathrm{ng}$ spike of caffeine. 60

Figure 3.3: LC-MS of a 5 ng spike of caffeine. 60

Figure 3.4: Caffeine AUP (blue) and RT (orange) reproducibility as an internal standard.

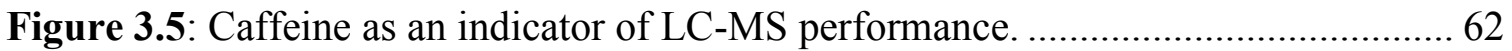

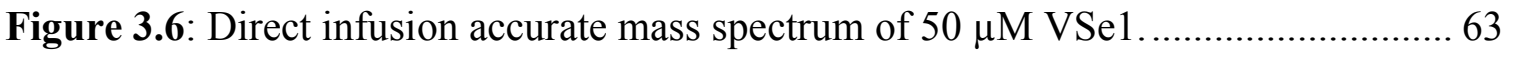

Figure 3.7: Direct infusion accurate mass spectrum of $50 \mu \mathrm{M}$ VSe7 $7 . \ldots \ldots \ldots \ldots \ldots \ldots \ldots \ldots \ldots . . . \ldots 3$

Figure 3.8: The proposed mechanism through which VSes lose the $\mathrm{HCl}$ neutral fragment. 66

Figure 3.9: Product ion scan of the 193 Th VSe1 Fragment $(50 \mu \mathrm{M})$. 67 
Figure 3.10: LC-MS of a $500 \mu \mathrm{M}$ VSe1 solution beyond the dynamic range of the detector. 68

Figure 3.11: LC-MS analysis of a $12 \mu \mathrm{M}$ VSe1 solution that is suitable for QC experiments 68

Figure 3.12: LC-MS analysis of a $4.8 \mu \mathrm{M}$ VSe7 solution that is suitable for QC experiments. 69

Figure 3.13: VSe1 frozen long-term stability. 70

Figure 3.14: VSe7 frozen long-term stability. 71

Figure 3.15: VSe7 stability under experimental egg conditions. 72

Figure 3.16: VSe7:Caffeine AUP Ratio standard curve. 73

Figure 3.17: VSe7 absolute quantitation method validation of the internal standard and diluents. 75

Figure 3.18: Validation of VSe7 resolubilization time in aqueous diluent.................... 76

Figure 3.19: VSe7 stability in DMSO solution at room temperature. 77

Figure 4.1: Product ion scan of MD01145 (compound 10) with a collision energy of 25

$\mathrm{eV}$ 81

Figure 4.2: Validation of the MD01145 MRM transitions with LC-MS........................ 82

Figure 4.3: MD03182 Standard curve correlating concentration with MRM AUP ........ 82 Figure 4.4: Improving the elution profile of MD03182 by switching to a sharper gradient B. 83

Figure 4.5: Quenching VSes with 5\% FAw was effective in limiting further degradation in plasma 86 
Figure 4.6: Observing the plasma-induced degradation of three VSes simultaneously

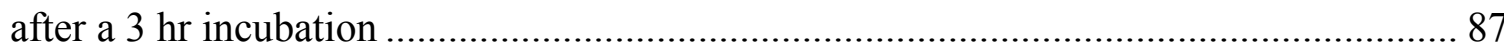

Figure 4.7: Time-course degradation of VSe1 and MD01145 in plasma...................... 90

Figure 4.8: Summarizing the significance of each MD01145 functional group ............ 91

Figure 4.9: Relative quantitation of VSes from blood samples obtained from IP injection 105

Figure 4.10: Relative quantitation of VSes from tumour samples obtained from IT injection 105

Figure 4.11: The detection of VSe-specific metabolites using EMS and NL scans 107

Figure 4.12: Possible metabolites conjugating to VSes based from accurate mass scans 108

Figure 4.13: The VSe-GSH adduct forms quickly but is short-lived in blood 110

Figure 4.14: The unidentified metabolite accumulates in tumour tissues over time but degrades in blood 110

Figure 5.1: The migration of proteins to their pI along an IEF gradient. Adapted from [3]

Figure 5.2: DIGE shows proteome expression changes upon drug treatment. Adapted from [4] 115

Figure 5.3: Qualitative assessment of protein contents in MD01145-treated and untreated HEK293 fractionated proteomes. 117

Figure 5.4: DIGE showing changes to the HEK293 F1 and F3 proteomes upon MD01145 treatment 118 
Figure 5.5: DIGE showing changes to the HEK293 F2 and F4 proteomes upon MD01145 treatment

Figure 5.6: MASCOT results identifying $\mathrm{PK}$ as the upregulated $\mathrm{F} 1$ protein spot 120

Figure 5.7: MASCOT results showing almost complete sequencing of the 33-43 Nterminus PK peptide

Figure 5.8: MASCOT results showing annexin A2 as the downregulated F3 protein spot

122

Figure 5.9: MASCOT results showing partial sequence coverage of the 105-115 annexin A2 peptide 123

Figure 5.10: Light microscope images (10X) of 786-0 cells treated with $50 \mu \mathrm{M}$ MD01145 (left) and $50 \mu \mathrm{L} \mathrm{MeOH} \mathrm{(right)} \mathrm{prior} \mathrm{to} \mathrm{harvesting}$ 124

Figure 5.11: Albumin standard curve using the BCA assay. 1:10 diluted cell lysate absorbances are indicated 125

Figure 5.12: DIGE comparing 786-0 proteomes upon VSe treatment. Horizontal streaking is indicative of poor protein IEF. 126

Figure 5.13: Qualitative assessment shows negligible protein loss during spin filtration 126

Figure 5.14: DIGE comparing 786-0 proteomes upon VSe treatment. Poor rehydration of the proteins and uptake into the IEF strip may account for the low number of spots .... 127 Figure 5.15: MD01145 quantitation in cell culture media shows potential saturation of the VSe target 128

Figure 5.16: The active and inactive MD01145 probes used for sepharose bead conjugation. 129 
Figure 5.17: The coupling of MD01145-based probes to free amine sepharose beads through carboxylic acid activation 130

Figure 5.18: VSe targets pulled down using active, inactive and unconjugated beads after a $12 \mathrm{hr}$ incubation with lysate uninfected and VSV $\Delta 51$ incubation 132

Figure 5.19: VSe targets pulled down using an active probe after $1 \mathrm{hr}$ incubation with uninfected and VSVD51 infected lysates....... 132

Figure 5.20: Final washes contained no observable proteins while the lysates proved the presence of numerous proteins available for VSe targeting 133

Figure 5.21: MASCOT results for GSTp1 represented by two class-specific peptides 134 Figure 5.22: Fragmentation of the first GSTp1 peptide to produce $90 \%$ of the $y$-ion series with consistent mass accuracy 134

Figure 5.23: MASCOT results for PR1 represented by three unique peptides. 135

Figure 5.24: MASCOT results for PR2 represented by two unique peptides. 135

Figure 5.25: GSTp1-VSe1 reaction kinetics analyzed by LC-MS shows no signs of covalent inhibition 137

Figure 5.26: MASCOT results of a GSTp1 digest showing detection of the active site Cys47 138

Figure 5.27: Chemical structures of the probes used for click chemistry-based ABPP 139 Figure 5.28: Copper catalyzed click chemistry cyclo-addition reaction 139

Figure 5.29: General design of a click chemistry-based ABPP target pull-down ..... 139

Figure 5.30: Proteins pulled down from 786-0 lysate using the MD03182-based probe for $60 \mathrm{mins}$ 141 
Figure 5.31: Qualitative assessment of various bead washing conditions all show

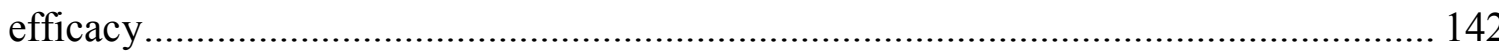

Figure 5.32: VSe1-based probe target pull-down from 786-0 cell lysate using streptavidin-linked sepharose beads 143

Figure 5.33: MD03182-based probe target pull-down from 786-0 cell lysate using streptavidin-linked sepharose beads 144

Figure 5.34: General design of a click chemistry-based pull-down using rhodamine fluorescent tagging 144

Figure 5.35: ABPP VSe target identification from 786-0 cell lysate using rhodamine fluorescent tag. 


\section{Chapter: Introductory Topics}

\subsection{Viruses}

Viruses are non-living infectious agents that are dependent on host cell machinery for their replication and propagation. In general, viruses consist of three main components: a genome, a protein capsid, and in some cases a lipid envelope. The genetic material is composed of either DNA or RNA that is observable in double-stranded and single stranded formats. For added diversity, the genome can be circular, linear or segmented with each section encoding for a unique viral protein. Capsid proteins encapsulate and protect the genetic material against the harsh environments within the host including extreme $\mathrm{pH}$, free radicals and degradative enzymes. Additionally, the capsid is capable of signalling for genome insertion, uncoating and facilitating host cell adhesion. The outer viral envelope includes lipids and carbohydrates derived from the host membrane and viral glycoproteins responsible for improving viral penetration and immune evasion [1].

Infection is initiated when a virus particle binds to a receptor on the host cell surface, or enters the cell by fusion of the viral envelope with the host lipid bilayer. The genetic material penetrates the plasma membrane and enters the cytoplasm where it is unsheathed and available for replication. DNA viruses transcribe into mRNA while RNA viruses use reverse-transcriptases to synthesize complementary DNA templates that can be used in a similar manner. During the lytic life cycle, the viral proteins hijack the cellular protein translation machinery to assemble new virus particles that can bud from the host and cause cell lysis [1]. Viruses can enter the lysogenic cycle which involves incorporating the viral genome into the host genome to ensure persistent transcription of 
viral proteins. Viruses can also selectively down-regulate gene expression to enter latency for the purposes of immune system evasion.

Throughout history, viral infections have lead to countless disease outbreaks and fatalities. Since its isolation in 1983 [2], clinical virus research has heavily focussed on human immunodeficiency virus (HIV) which annually kills an estimated 1.5 million people worldwide [3]. The most recent outbreak with significant media coverage was the Ebola virus in Western Africa that caused more than 6,000 cases and 3,000 deaths by September 2014 [4]. Despite these alarming statistics, host cells are efficient in combating viral infection through diverse mechanisms. The adaptive immune response produces $\operatorname{IgM}$, followed by $\operatorname{IgG}$ antibodies against surface antigens to block the entry of circulating virus particles. The innate immune response includes natural killer (NK) cells that are attracted by chemokines to the infection site and induce apoptosis of cells presenting viral antigens. There are multiple levels of the antiviral immune response that have been extensively reviewed [5].

At the intracellular level, one of the most effective antiviral responses is the interferon (IFN) pathway; signaling proteins that are secreted to boost the antiviral responses of neighbouring cells to prevent further spread of the infection. Initially, double-stranded viral RNA is detected and leads to the phosphorylation and subsequent

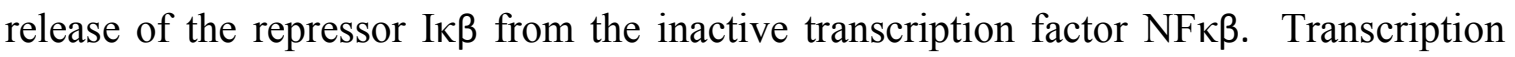
factor activation results in its translocation to the nucleus where it initates IFN gene transcription [6]. When IFN binds to a neighbouring cell receptor, JAK/STAT signaling cascades upregulate more than 300 IFN-stimulated genes that are normally minimally expressed or quiescent (Figure 1.1). Many of these gene products create positive 
feedback loops that further increase the expression of IFN and pattern-recognition receptors, however few products have been directly linked to antiviral activity. Antiviral gene products that have been more extensively characterized are: IFN-stimulated protein of 15kDa (ISG15), GTPase myxovirus resistance-1 (Mx1), ribonuclease L (RNaseL) and protein kinase R (PKR). ISG15 is an ubiquitin homologue that conjugates to host immune response proteins such as STAT1 and IRF3 to prevent their viral-induced ubiquitination and subsequent degradation. Additionally, ISG15 can be conjugated to viral proteins such as HIV-1 proteins Gag and Tsg101 to prevent their ubiquitination and stop virion release from the host [7]. Mx1 associates with viral polymerases to limit viral gene transcription while also binding to the endoplasmic reticulum membrane to survey for budding capsid proteins and prevent their trafficking. RNaseL is similar to the endogenous dicer complex since it cleaves double stranded viral RNA genomes to inhibit their translation. PKR is quiescent under normal conditions, but the binding of double-stranded viral RNA leads to enzyme activation. PKR phosphorylates EIF2 $\alpha$ which sequesters GDP and prevents the use of GTP in viral protein translation [8]. Overall, IFNs are an effective defense mechanism against viral infection by restricting infected cell growth, limiting viral propagation and increasing the immunogenicity of infected cells [6]. 


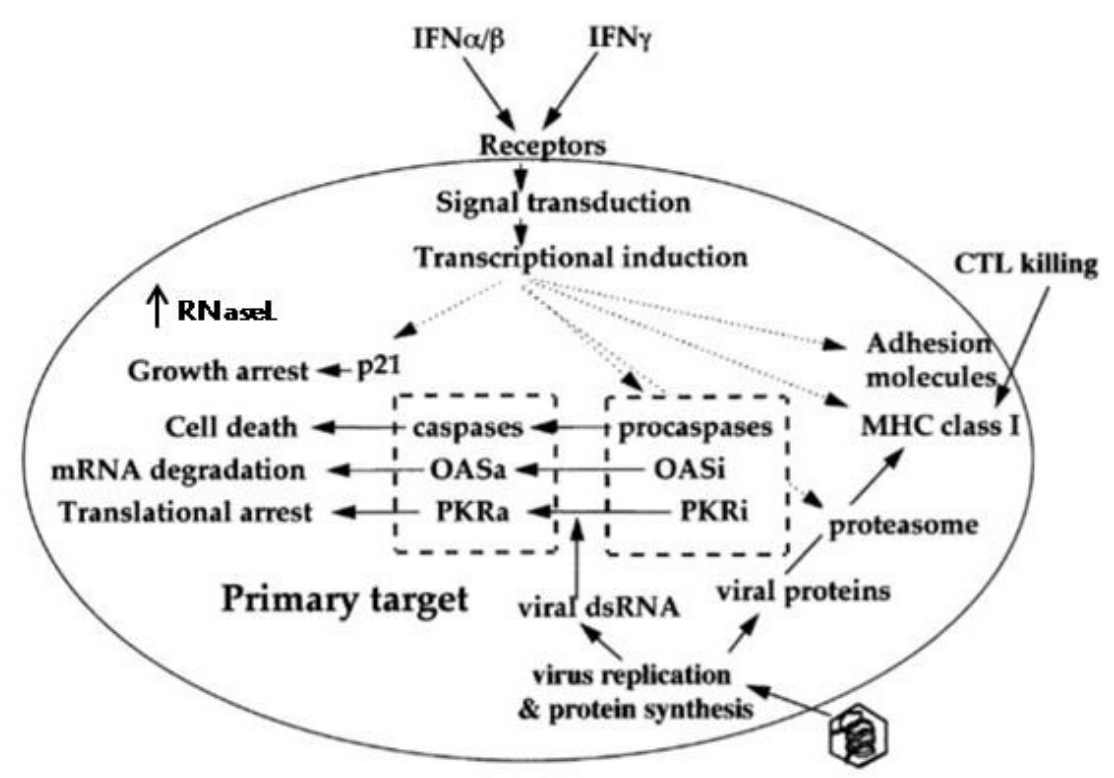

Figure 1.1: IFN response in neighbouring cells prepares them for viral infection. Adapted from [6]

Viruses are frequently undergoing silent mutations due to the rapid rate of genome replication and poor proof-reading functions of viral polymerases; ultimately amounting phenotypes with increased virulence. As a result, viruses have developed fascinating measures to counteract the cellular antiviral response. For example, the Nterminus of an influenza-B virus non-structural protein has developed the ability to sequester IGN15 and prevent it from signaling for viral degradation [8]. The Epstein-Barr virus EBNA-1 protein is capable of decreasing proteasomal degradation of other viral proteins to prevent their presentation by MHC molecules [9]. Meanwhile the EBNA-3A protein is degraded to supply peptides for MHC presentation that are recognized by NK cells as native [10]. Improvements in immune response evasion arise from the high rate of point mutations that generate countless epitopes of immunogenic peptides. These "escape mutants" can overwhelm and divert the immune response from focussing on a specific antigen. Improved immune evasion is also achieved by the Epstein-Barr virus 
which secretes viral proteins that are homologous to the immunosuppressive cytokine IL$10[11]$.

Regardless of the negative reputation viruses have gained, they can also be used for beneficial applications. Mutations to virulence factors can lead to a restricted host cell range or attenuation; meaning the virus is still viable but is far less virulent and has a lower capacity to replicate. Attenuated viruses form the basis of several virus-based therapeutic platforms because their virulence power has been harnessed in recent decades for applications such as vaccines, oncolytic virotherapy $(\mathrm{OVt})$ and gene therapy vectors.

\subsection{Vaccine Production}

Vaccination is a form of lasting protective immunity and is arguably one of the most significant technological developments of the past century. Smallpox and poliovirus, once deadly diseases, have been all but eradicated from immunized human populations [12]. An attenuated pathogen, or part thereof, is administered to the patient and the adaptive immune response generates antibodies that reduce the risk of future infection. Different vaccine types are available that vary by downstream processing, administration and regulations. Live attenuated vaccine efficacy is dependent on decoupling the virulence from the desired protective immunity [13]. Once this has been achieved, the live vaccine type is considered to be the most effective because it includes three important components: an adaptive response-stimulating antigen, adjuvant effects and efficient delivery due to the virus' natural invasiveness. These advantages come at the risk of reversion to full virulence bringing the vaccine's safety into jeopardy. Inactivated vaccines contain virus particles that have been killed by chemical treatment 
(such as formaldehyde), thus relieving the risk of live vaccine virulence coming at a cost of reduced potency. When a specific viral immunogen is known and well-characterized, such as the tetanus toxin, purified protein vaccines are used for their ease of production and improved safety [13].

Vaccine viruses are typically produced in fertilized chicken eggs and the production strategy has remained largely unchanged since its inception more than 60 years ago [14]. Few modifications have been made to the downstream processing of vaccines including: the use of detergents for improved antigen purification and the use of high growth reassortant strains to improve the yield of newly identified influenza strains. As a result of their great success, the demand for vaccines is steadily growing to raise concerns of exceeding the stagnant annual supply. Manufacturing companies are experiencing difficulties keeping up with the growth in demand because of increasingly stringent safety guidelines in the face of reduced profit. The egg-based method is disadvantageous because it is time-consuming, and requires large amounts of space and introduces the risk of egg protein contamination to which $3 \%$ of children are allergic [14].

Accordingly, alternative strategies have been developed by manufacturing companies, with cell culture-based production garnering the most attention. The propagation of vaccinia virus in cell cultures was first demonstrated in 1949 by Enders \& Weller [15]. Primary cell lines from primate embryos were used in the production of inactivated poliovirus but it was not feasible over the long-term due to the short cell lifespan and risks of simian virus contamination. The development of continuous cell lines was necessary for sustained vaccine production, but they were initially neglected 
due to their tumorigenicity. A World Health Organization study in 1986 [16] deemed the use of continuous cell lines with theoretical infinite lifespans as acceptable for the production of vaccines and other biologics. As per regulations, it must be validated that finished vaccine products do not contain tumorigenic live cells and host cell DNA is limited to $10 \mathrm{ng}$ per dose and degraded to no larger than $200 \mathrm{bp}$ in size. Vero cells (African green monkey kidney cell line) are used most frequently based on their preexisting and long-withstanding protocols; although Madin-Darby canine kidney (MDCK) and PER-C6 cell lines are gaining recognition as possessing more potential [15].

Continuous cell cultures provide numerous advantages in comparison to eggbased production strategies. The latter lacks flexibility since it can't be scaled up on sudden notice to meet changes in demand or virus serotype. Cell cultures require shorter lead-up times and are not bound by limited egg supplies that must be ordered up to a year in advance. From a regulatory perspective, cell cultures are more favourable because of regular DNA-sequencing and maintenance of cell types in addition to previously developed virus purification techniques from research laboratories. Furthermore, the cell culture-based method requires less space, is more robust, eliminates the possibilities of egg protein contaminants and are capable of growing avian influenza strains that are not feasible via chicken embryos [14]. Recently, method development has increased the yield of cell culture-based influenza vaccines to be on par with egg-based methods [17]. To demonstrate the growing confidence in this technique, Novartis opened the first American influenza vaccine manufacturing plant that was dependent on Vero cell cultures in 2006 [17]. 
New vaccine technologies are emerging to improve upon the shortfalls of modern vaccines: ease of development, potency and safety. Various formats are now available for cell culture-based production including roller bottles, stacked-array flasks and continuous-use bioreactors that differ in regulation, efficiency and scalability. Considerable effort has gone into developing serum-free media platforms to limit the use of animal products as well as introduce growth supplements that will boost cell defenses against virus-induced apoptosis for improved yields [17]. Gene-based vaccines are under development that involves the insertion of antigen-encoding DNA into cells for prolonged antigen expression while avoiding the risks of using live viruses. Viral-based gene delivery is also possible but these two approaches require improvements in immunogenicity and genetic stability [18]. Also in the developmental phase are virus-like particles that are safe with enhanced immunogenicity. These are composed of denselypacked self-assembling arrays of capsid proteins that do not contain genetic material, but require more extensive preparation. Plant-based vaccine production is appealing because the expressed recombinant proteins are edible but are restricted to protein-based vaccines. This enables self-administration and antigens can be grown at pre-existing agricultural centres. Oral induction of the immune response mimics the induction caused by the majority of pathogens at mucosal surfaces. Further studies will need to address plant glycosylation patterns that may affect antigen stability and immunogenicity. Finally, protein vaccines require priming the immune response for vaccine potency because they lack additional immunogens included in the full virus structure. Adjuvants and innate immune potentiators are constantly being evaluated and added to vaccines to trigger inflammation [13]. 


\subsection{Cancer and Oncolytic Virotherapy}

Tumours arise from the unregulated proliferation of cells with cancer causing an estimated daily average of 210 deaths in Canada, which accounts for $29.9 \%$ of all Canadian mortalities [19]. Cumulative DNA damage caused by oxidation, chemical mutagens or viral infections is not corrected by underperforming sequence editing. Cancer arises from mutations that enhance cell proliferation and the mutational clone replicates prior to programmed cell death, known as apoptosis [20]. Cancers become pathogenic when they are not destroyed by the innate immune system that is responsible for surveying the body for abnormally replicating cells. There is a wide range of mutations that are associated with cancer that can be generalized by five mechanisms: independence from growth stimulatory signals, unresponsiveness to growth inhibitory signals, resistance to apoptosis, overcoming cellular senescence and the development of angiogenic potential [20].

Cancerous cells develop an independence from external growth factors through several oncogenic mechanisms. Chronic myelogenous leukemia is associated with the BCR-ABL fusion protein caused by a chromosomal translocation event. Individually, the $\mathrm{BCR}$ and $\mathrm{c}-\mathrm{ABL}$ gene products are serine and tyrosine kinases (respectively) but the tyrosine kinase is constitutively activated in the fusion product leading to upregulation of endogenous growth factors [21]. Other oncogenes such as Ras [22], cyclin D [23] and cmyc [24] are implicated in similar mechanisms that can upregulate expression of proangiogenic factors such as vascular endothelial growth factor (VEGF) and fibroblast growth factor (FGF-1). In the opposite sense, deactivating mutations to tumor suppressor 
genes such as p53 can also result in cancerous phenotypes. p53 acts as a genome guardian by surveying for abnormal DNA synthesis, telomere length (an indication of nearing senescence) and the inappropriate activation of oncogenes. The protein then initiates malignant cells apoptosis, however mutations to $\mathrm{p} 53$ eliminate critical contacts with DNA to reduce its binding activity [20]. The differences between cancerous and non-cancerous gene expression profiles allow for exploitative targeted cancer treatments.

Non-selective, traditional cancer therapies such as chemotherapy and radiation will be overshadowed in the coming decades by the development of new, selective treatments. Oncolytic virotherapy $(\mathrm{OVt})$ has emerged as a promising approach to tumour desturction. Oncolytic viruses (OVs) possess engineered mutations that allow for preferential tumour binding and infection. Tumour destruction is a product of either the virus lytic cycle or the expression of virus apoptosis-inducing genes (a form of gene therapy). Furthermore, OVs are designed to induce the expression of cellular antigens and cytokines that amplify the anti-tumoural immune response [25].

Multiple virus families have been attenuated through diverse strategies and evaluated for their potential as OVs including, but not limited to: herpes simplex virus, vaccinia virus and adenovirus. Infection selectivity is the principal characteristic of OVs that needs to be optimized for clinical safety and is being tested from different angles. For example, the replication of the adenovirus E1 gene has been engineered to be dependent on the increased expression of human telomerase reverse transcriptase in cancer [25]. Adenovirus target cell binding is enhanced by modifying the hypervariable loop of the hexon protein but it does not bind to normal cell surfaces [26]. Ras, an upregulated GTPase cancer biomarker is exploited by the herpes simplex OV. In addition to Ras, its 
transcription factor Elk is upregulated in and preferentially binds the OV gene encoding for a protein required for viral replication [27]. Moreover, oncolytic adenoviruses have been developed with micro-RNA binding sites that limit liver toxicity without compromising anti-cancer activity [28].

Obstacles surrounding the delivery of OVs to the target cells must be overcome if OVt is going to fully meet its efficacy potential. Neutralizing components of serum, such as antibodies, significantly reduce the half-lives of OVs. The delivery of viruses via engrafted stem cells or surface coating them with polyethylene glycol polymer have been investigated to improve blood half-life. Intravenous administration of OVs leads to rapid clearance by the liver and spleen and poor tumour penetration [25]. Transferring OVt technology from the pre-clinical laboratory to clinical applications has been accompanied with a loss in efficacy. Nonetheless, the recent emergence of approved virus-based cancer therapeutics have shown great promise in the field of OVt research. In 2005, the oncolytic adenovirus H101 OV was approved in China for the treatment of head and neck carcinomas [29]. Notably, the T-Vec strain of herpes simplex virus-1 that expresses a macrophage stimulating factor in melanoma is expected to be approved in North America based on favourable phase III clinical trial results [30]. A combined Maraba virus and adenovirus treatment is currently in clinical trials at the Ottawa Hospital [31].

Similar to viruses, cancer cells frequently undergo mutations that often result in the loss of the IFN antiviral pathway functionality, which can be exploited by oncolytic strains of vesicular stomatitis virus (VSV). The rhabdovirus VSV genome consists of five consecutive genes that encode for the nucleoprotein (N-protein), phosphoprotein ( $\mathrm{P}-$ protein), glycoprotein (G-protein), matrix protein (M-protein) and large polymerase (L- 
protein) [32]. VSV has an RNA genome that replicates solely in the cytoplasm; an added advantage over DNA-based viruses that replicate in the nucleus and could lead to mutations once integrated into the host genome. The genome is enveloped by the Mprotein capsid while the G-protein facilitates binding to phosphatidylserine (causing a universal animal tropism) and proceeds to endocytosis. The acidic endosome environment leads to fusion of the viral membrane with the endosome membrane and insertion of the genome. Once translated, the N-protein, P-protein and L-protein form a RNA polymerase complex to propagate genome transcription [33]. The M-protein is versatile in that it helps the assembly of new viruses, induces apoptosis and regulates the transcription of viral genes at different phases of infection. For example, the N-terminal domain interacts with Nedd4 enzyme that causes assembled viral budding in the late stages of infection. In the early stages, the M-protein plays a vital role in dampening cellular antiviral responses. It inhibits cellular transcription once the viral polymerase complex has been established and importantly, it blocks the transport of IFN mRNA from the nucleus by interacting with nucleoporin protein Nup98 (Figure 1.2A) [33].

Within this dissertation, VSV $\Delta 51$ was regularly studied in vitro for its potential as an OVs. VSV 551 M-protein suffers from a Met-51 deletion mutation leading to severely attenuated growth in normal tissues without compromising tumoural infection. The mutated M-protein is unable to tightly interact with Nup98 and block the transport of IFN transcripts which ultimately eliminates the infection in normal cells with functional antiviral pathways (Figure 1.2B). The mutant M-protein attempts to block the transcript transport in tumour cells but the IFN gene product is not functional, thus permitting selective infection in tumours (Figure 1.2C) [32]. The genetic heterogeneity amongst 
tumour cells with functional IFN responses limits the intratumoural spread of VSV $\Delta 51$. Combination therapies have been investigated to overcome this constraint, such as adding an oncolytic poxvirus that secretes an IFN antagonist to complement VSV $\Delta 51$ treatment [25]. Another combinational therapy route is using small molecules to propagate infection. Inhibitors of histone deacetylases or rapamycin complex-1 dampen the innate antiviral response by decreasing the production of IFN [25].
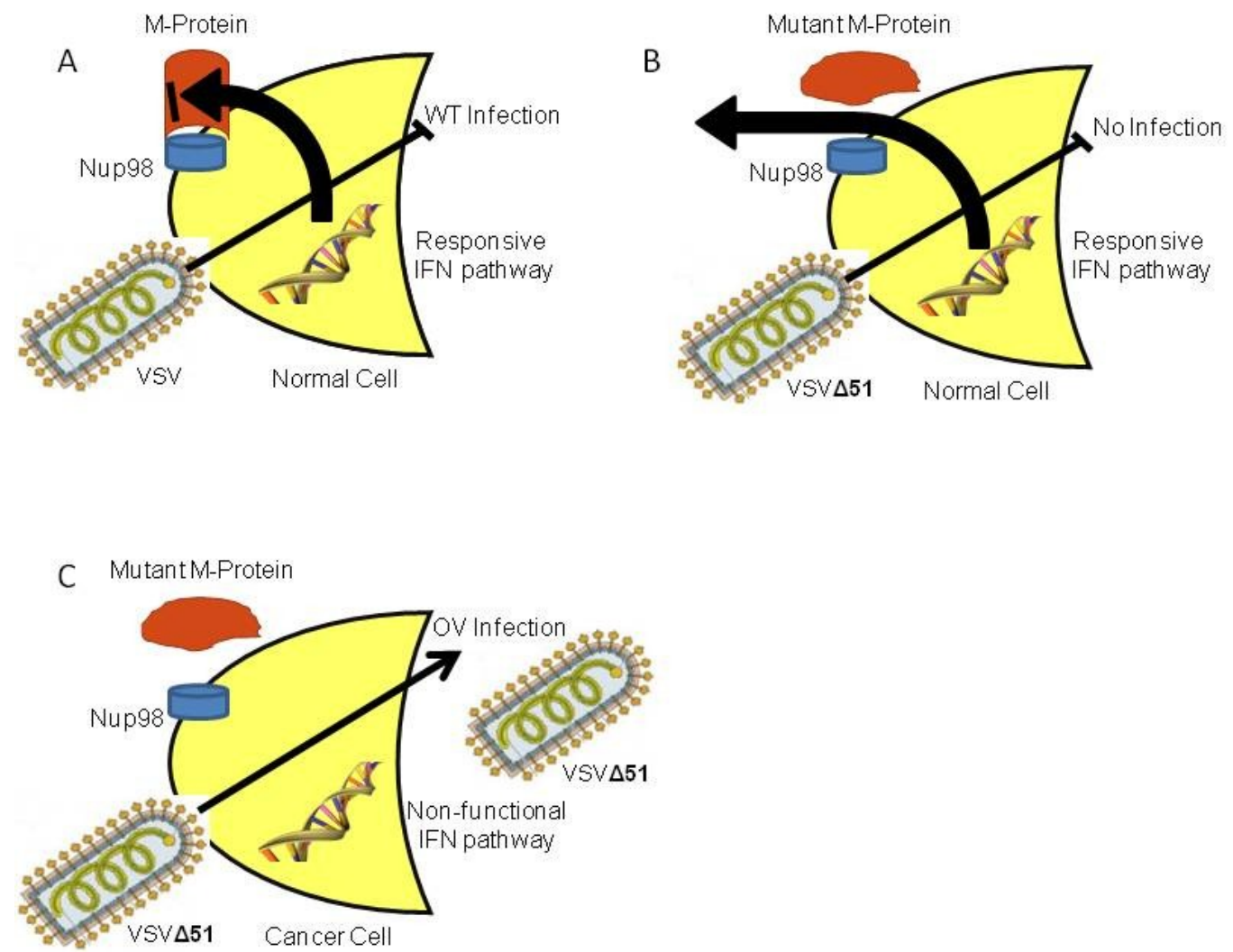

Figure 1.2: Wild-type VSV M-protein blocks the transport of IFN to the cytoplasm (A). VSVA51 selectively infects cancer cells based on this principle (B \& C).

\subsection{Viral Sensitizers}

The aforementioned attenuated viral applications are either unable keep up with growing demand or are limited in their efficacy by one root problem: the propagation of viral infection. Cells within an embryonated chicken egg are killed by viral infection, 
therefore the amount of vaccine produced from an egg is finite. Cancer cells are frequently mutating and as a result lose antiviral defense functionality, particularly the IFN pathway. The propagation of viral infection is limited from intratumoural genetic heterogeneity since an estimated $30 \%$ of host cells are OV-resistant [29]. A research group at the Ottawa Hospital Research Institute (OHRI) decided to investigate the use of small molecules to selectively enhance OV replication in tumours while preventing infection in non-cancerous tissues. Unlike the histone deacetylase or rapamycin complex1 inhibitors, a high-throughput in vitro screen of 12,280 compounds was performed in 2010 to identify compounds that enhance the growth of OV VSV $\Delta 51$ expressing red fluorescent protein (RFP) in 4T1 OV-resistant breast cancer cells [34]. Fifteen of the most active hits were termed viral sensitizers (VSes) (Figure 1.3). The most active VSe (VSe1, structure shown) amplified the viral titer 34-fold as demonstrated by fluorescence microscopy. To further validate this finding, VSe1 enhanced VSV $\Delta 51-R F P$ replication in other highly OV-resistant cancerous cell lines (Figure 1.4) such as colon CT26 cells, kidney 786-0 cells and U251 glioblastoma cells relative to virus. Most importantly, the safety of VSe treatment was supported because the normal fibroblast GM38 cell line remained OV-resistant in the presence of VSe1. Mechanistic studies revealed that IFNinducible antiviral gene transcripts were reduced by $96 \%$ upon exposure to VSe1 although the intracellular interaction partner remained unknown. VSe1 also selectively enhanced OV growth in ex vivo mouse and human cancerous tissues [34]. 

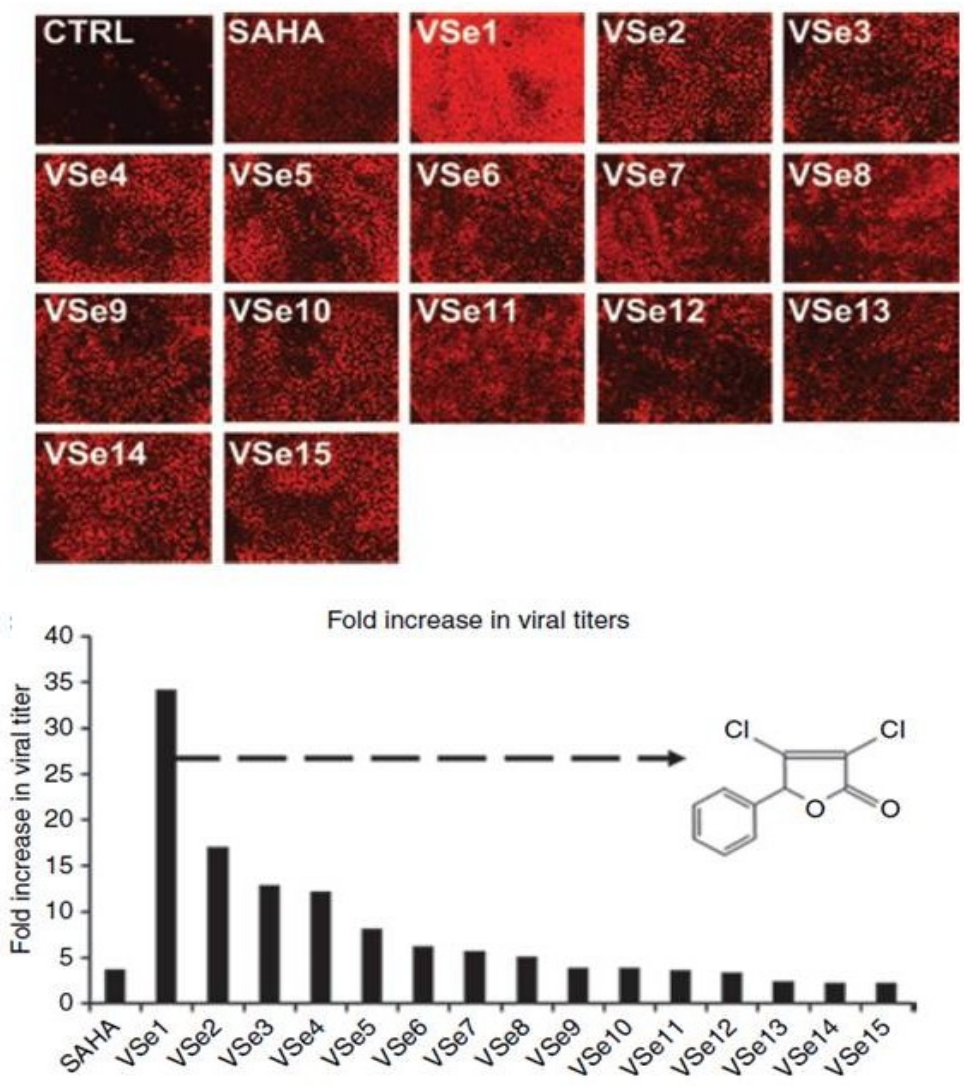

Figure 1.3: VSe1 was the most active compound by enhancing VSVA51-RFP replication in OVresistant $4 \mathrm{~T} 1$ cells $34-$ fold [34]

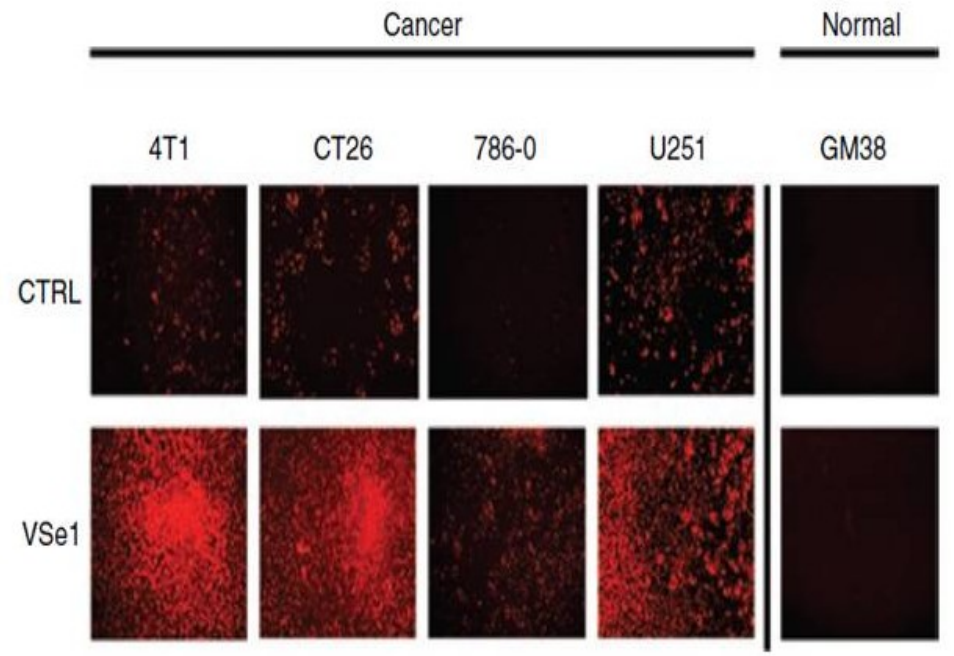

Figure 1.4: VSe1 selectively enhances OV replication in multiple resistant cancerous cell lines [34] 
VSes are promising first-in-class potentiators of attenuated viral growth that can improve upon aforementioned therapeutic applications. There are multiple benefits of using VSes in combination with current vaccine production strategies. Enhanced vaccine production not only will maintain the supply at a higher level than growing demand but also reduces the lead-up time and production time, which in turn lowers costs. This would be particularly useful during viral epidemics when large quantities of vaccine are suddenly required in a shorter period of time [12]. For OVt, VSes will provide the needed boost in viral propagation so that more tumour tissue can be selectively infected. Ideally, this will improve treatment rates and reduce the frequency of cancer recurrence [35].

As promising as these results were, VSe1 was found to be poorly tolerated by the cells (including non-cancerous cells) and highly unstable in aqueous media. More desirable physiochemical properties are needed for VSes to realize their potential in OVt and vaccine production. A collaborative effort between our research group, Jean-Simon Diallo's group at the OHRI and Chris Boddy's group at the University of Ottawa sought to further investigate VSes for improved properties.

\subsection{Mass Spectrometry}

The analyses of small organic molecules and biomacromolecules have been revolutionized by the development mass spectrometry (MS) technology. A century since its inception, the mass spectrometer has proven to be a versatile instrument that is applied to diverse facets of analytical science. It is used in clinical settings to screen newborns for inherited metabolic disorders [36], to determine the composition of extraterrestrial matter [37] and to date fossils by radioactive carbon-dating [38] and many more applications. 
J.J. Thomson's early work separating the isotopes of neon gas by mass-to-charge $(\mathrm{m} / \mathrm{z})$ (Th) ratio were credited as a ground-breaking study in the field of MS [39]. The technique was further improved by Ashton and Dempster who included the modern mass spectrometer designs still used today. A mass spectrometer consists of three components that are essential in the generation of a mass spectrum: an ionization source, a mass analyzer and a detector (Figure 1.5).

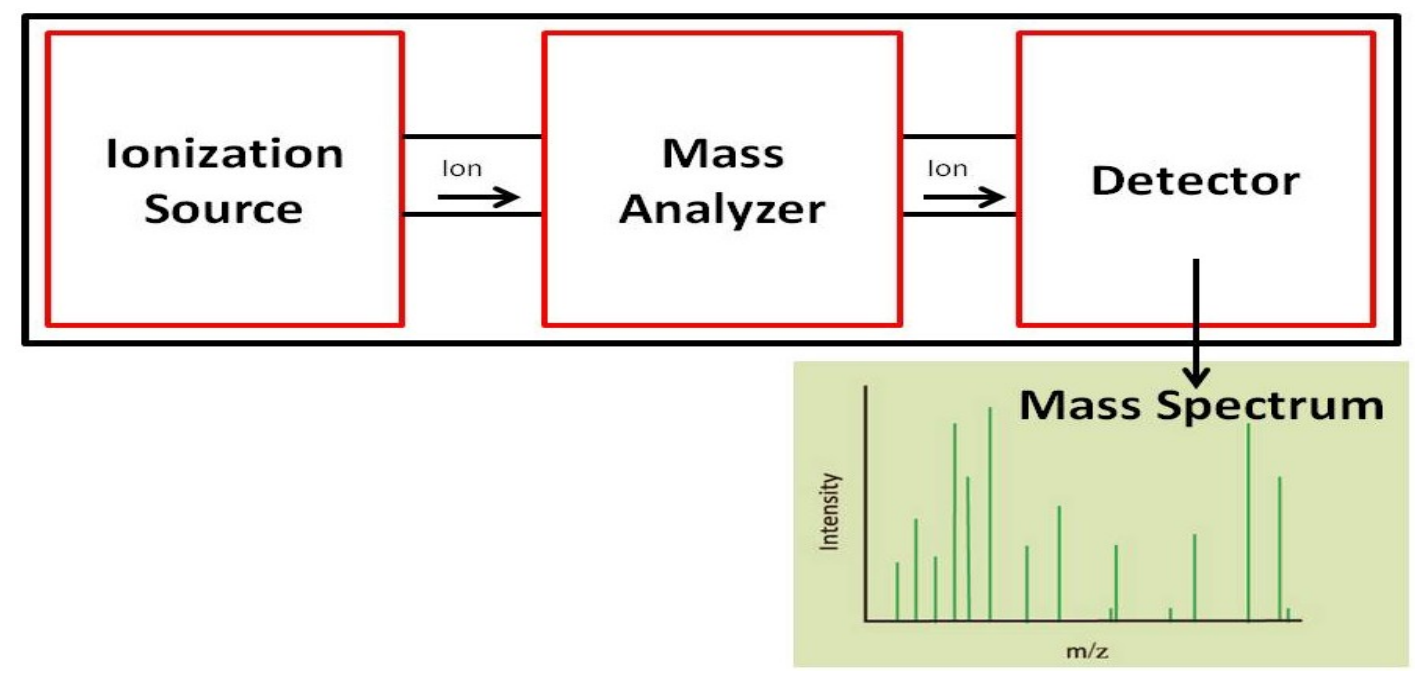

Figure 1.5: The essential components of a mass spectrometer

Great emphasis is placed on the ionization source because MS analysis requires analytes to be charged and gaseous. Electron ionization (EI) is suited to analyze highly volatile, non-polar compounds while moderately polar and volatile analytes are effectively ionized by atmospheric pressure chemical ionization (APCI). If the analyte is both highly volatile and polar, chemical ionization (CI) is the most appropriate mode of ionization [40]. Intact parent ions are difficult to detect using these modes because these ionization techniques invoke fragmentation; thus gaining the "hard" ionization label. The development of "soft" techniques such as matrix-assisted laser desorption ionization 
(MALDI) and electrospray ionization (ESI) by Tanaka and Fenn (respectively) significantly improved the analysis of large biomacromolecules to advance the fields of proteomics, metabolomics and lipidomics [41].

Within this dissertation, ESI is solely used to produce cations that were analyzed by positive ion mode MS. In ESI, samples are introduced at flow rate of microlitres per minute and oxidized by a highly-charged capillary. Liquid droplets are emitted from the capillary tip as a Taylor cone which leads to rapid droplet desolvation until the droplet's Rayleigh limit is reached. At this point, the repulsion forces of cations in close proximity overcome the droplet surface tension, causing it to become unstable and dissociate into desolvated cations in the gas phase. The ions are forced by an electric field to the anionic curtain plate and facilitated by an inert nebulizing gas (such as nitrogen) emitted from the tip (Figure 1.6) [42].

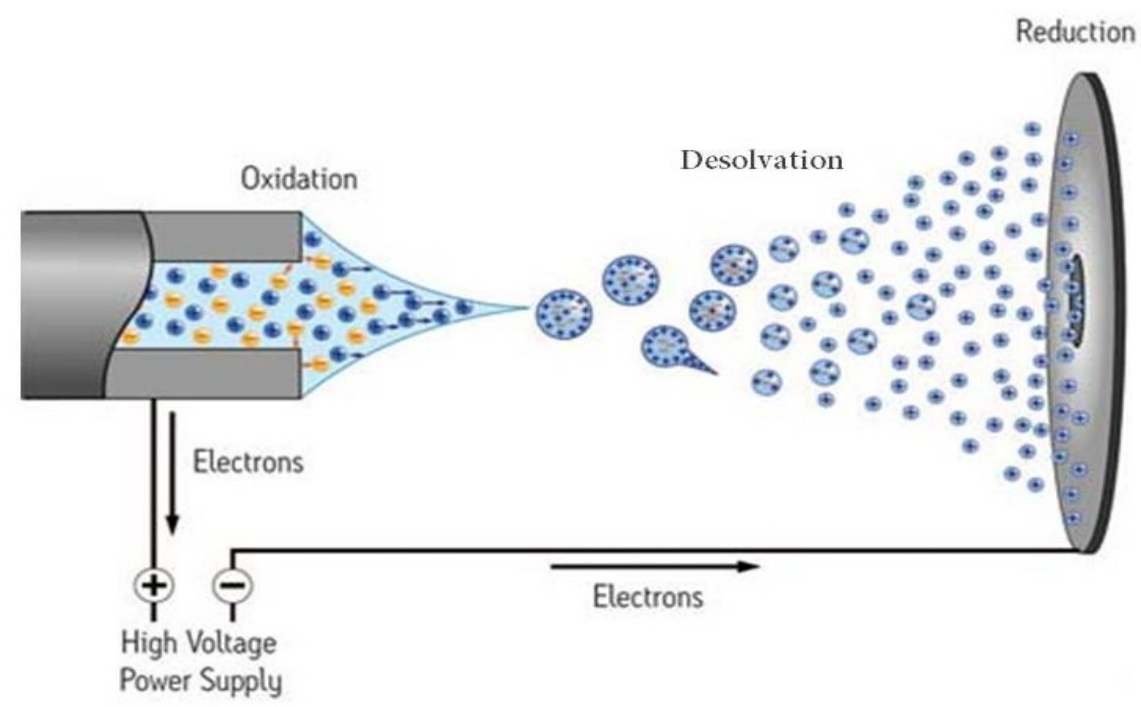

Figure 1.6: General schematic depicting electrospray ionization (ESI) [43]

Once the ions have entered the orifice of the MS curtain plate they encounter inert curtain gas that is gently flowed through the ion stream causing further desolvation. 
Consecutive compartments of the MS are under stronger vacuum conditions to eliminate the presence of unintended neutral molecules with which collisions may interrupt the ion stream. Upon entering vacuum conditions, the ion stream supersonically expands into a jet plume which is refocused using charged focusing rings and a quadrupole (q0) (Figure 1.7). A focused beam is necessary for ion transmission to the second MS component: the mass analyzer.

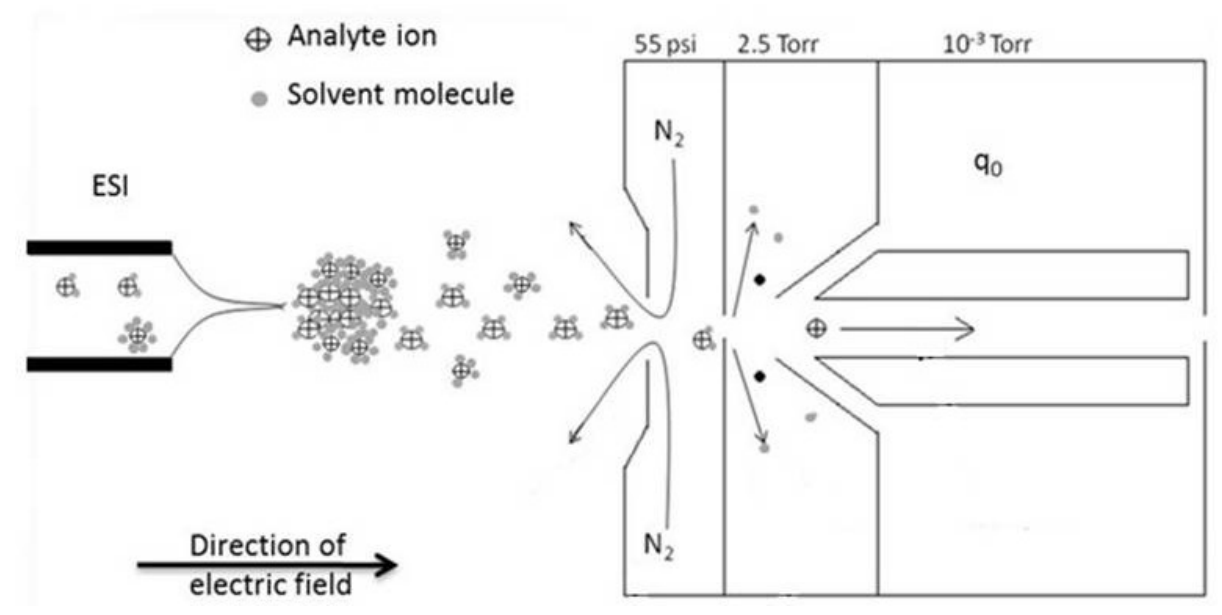

Figure 1.7: Layout of the QStar XL front end [44]

The various types of mass analyzers are constantly undergoing improvements in terms of resolution, ion transmission, and mass range. In this dissertation, three types of mass analyzers were used: quadrupoles and a time-of-flight (TOF) analyzer in the QStar XL mass spectrometer, while the 4000 Qtrap mass spectrometer contained quadrupoles and a linear ion trap (LIT).

Quadrupoles ideally consist of four hyperbolic rods. Oppositely positioned rods are electrically connected and carry the same charge polarity (Figure 1.8). An alternating current (AC) radio frequency is applied to each pair of rods and the polarities are cycled to create a rapidly alternating electric field which influences the ion beam's spiralling 
path along the quadrupole. A direct current (DC) potential can be superimposed onto the poles that allow for the selective transmission of resonant ions with a prescribed $\mathrm{m} / \mathrm{z}$. If the electric field increases the amplitude of the ions' motion in the quadrupole's $x-y$ plane, the ion trajectory becomes unstable and collides with the quadrupoles to deny transmission. If the DC dominates the RF, heavier ions are focused to the center of the quadrupole thus permitting their transmission and eliminating the low $\mathrm{m} / \mathrm{z}$ ions. If the $\mathrm{DC}$ field is weaker than the RF field, heavy ion trajectories are destabilized. Quadrupoles that act in RF-only mode transmit all ions to the detector (a full mass scan) but the DC potential allows for quadrupoles to act as mass filters [45].

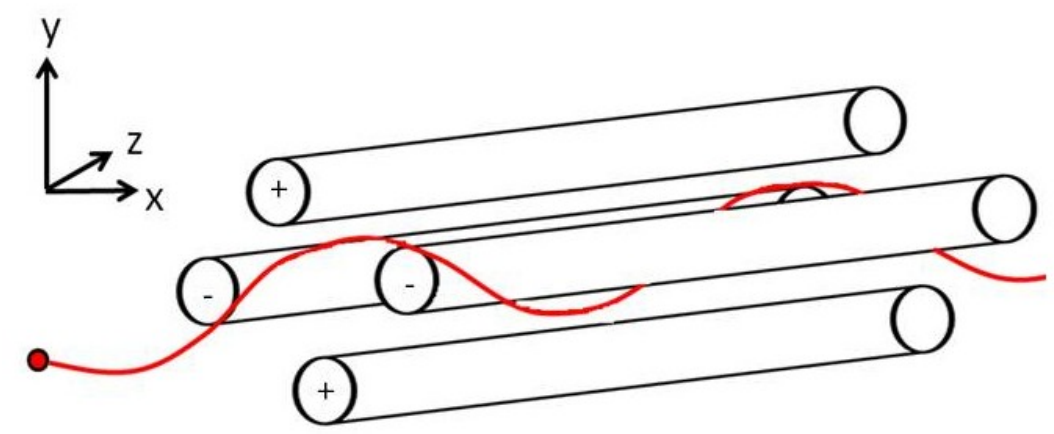

Figure 1.8: The alternating RF electric field on the quadrupole electrodes control ion motion and transmission

Tandem MS is the use of consecutive quadrupoles which permits the selective transmission of ions and their respective fragments that can be analyzed by different scanning modes (Figure 1.9). During a product ion scan, the RF and DC ratio is adjusted to select a specific ion $\mathrm{m} / \mathrm{z}$ that is transmitted from the first quadrupole (Q1) to a second quadrupole (q2) operating in RF-only mode. $\mathrm{q} 2$ is the collision cell where the transmitted ions are fragmented by collision-induced dissociation (CID) which involves passing a the ion beam through a chamber of neutral inert gas molecules (nitrogen or argon) through q2 
and was used in both the QStar XL and 4000 Qtrap mass spectrometers. The kinetic energy of the incoming ion beam can be adjusted via the potential difference between $\mathrm{q} 0$ and q2 to cause high-energy collisions with the collision gas. Such collisions cause internal parent ion energy to be translated to bond fragmentation into daughter ions that travel to a third quadrupole (Q3) that is sequentially scanning fragment $\mathrm{m} / \mathrm{z}$ by ramping the DC potential. The detection of daughter fragments from a product ion scan can reveal analyte structural information. In a precursor ion scan, all ions are sequentially scanned to $\mathrm{q} 2$ and specific ions that produce a prescribed fragment are detected to provide molecular class information. Similarly, a neutral loss (NL) scan sets a specific mass difference between Q1 and Q3 that are cycling through a prescribed mass range to identify members of compound classes that fragment in a similar manner. Selected, or multiple reaction monitoring (MRM) applies a DC potential to both Q1 and Q3 and a specific parent ion and daughter ion mass transition is selectively transmitted to the detector. The specificity of the method eliminates ramping of the DC that causes increased duty cycle time and losses in sensitivity.

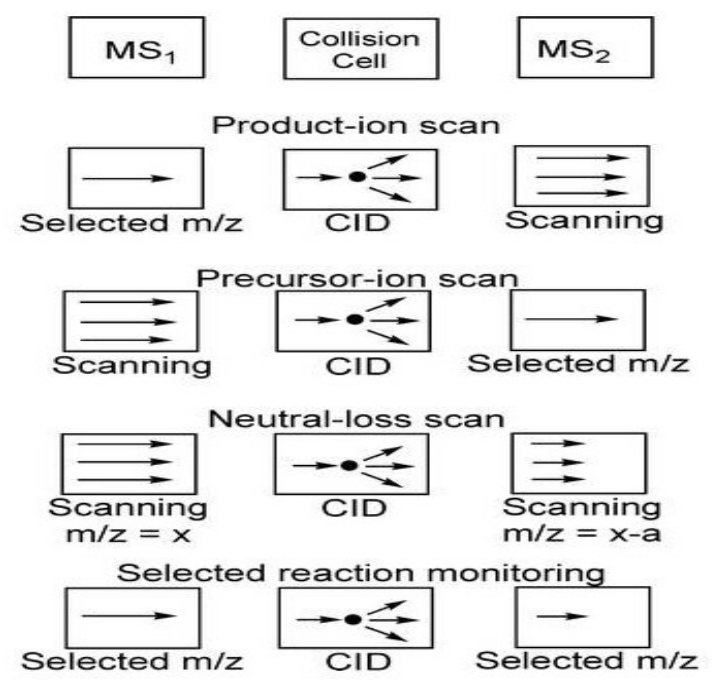

Figure 1.9: The various scanning modes enabled by tandem MS [46] 
In the QStar XL mass spectrometer, Q1 is followed by the q2 collision cell and a TOF mass analyzer (Figure 1.10). TOFs use a pulsed magnetic field to accelerate ions into a drift region under strong vacuum conditions. Smaller ions will travel faster than larger ions over the fixed distance so that travel time is correlated with $\mathrm{m} / \mathrm{z}$. TOF detectors provide enhanced resolution, ion transmission and analysis speed in comparison to quadrupoles [45]. TOF resolution is affected by ions arriving at the pulser at slightly different times; which can be corrected by collisional dampening. Collisional dampening is similar to the collision cell in that a neutral inert gas is used to slow down the ion beam to focus them into narrower ranges of time. Resolution is also improved using a reflectron ion mirror consisting of electrode rings that produce increasingly stronger magnetic fields. Pulsed ions are reflected back in the opposite direction towards the detector which increases resolution two-fold: it doubles the fixed distance that ions are separated by TOF and the mirror corrects for slight variances in TOF arrival time that were not corrected by collisional dampening (Figure 1.11) [47]. Since TOF mass analyzers are incapable of acting as mass filters, precursor, NL and MRM scanning modes are not permitted.

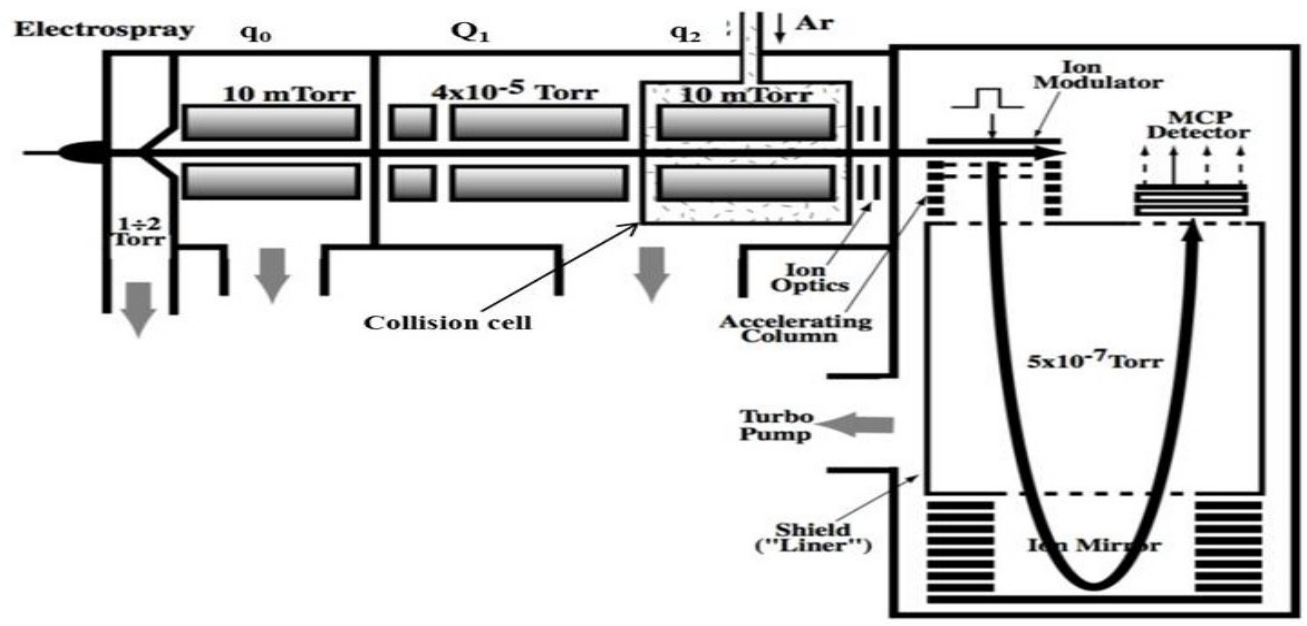

Figure 1.10: Schematic diagram of a QStar XL QqTOF mass spectrometer [44] 


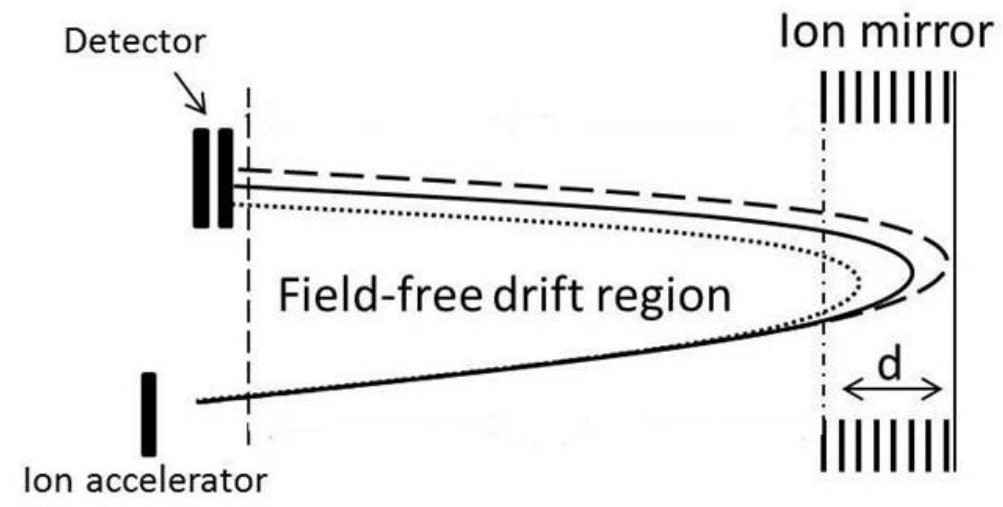

Figure 1.11: An ion mirror in a TOF mass analyzing corrects ion trajectories [48]

In the 4000 Qtrap MS, the first two quadrupoles are followed by a linear ion trap analyzer (LIT). The trap consists of a quadrupole with two end electrodes connected to an alternating current (AC) generator [49]. The RF traps the ions radially while the $\mathrm{AC}$ causes the ion cloud to be trapped along the electrode axis for longer periods of time which leads to enhanced sensitivity. Once ions resonate with the RF, they acquire substantial axial velocity to overcome the exit lens barrier and are ejected axially (Figure 1.12) $[50]$.

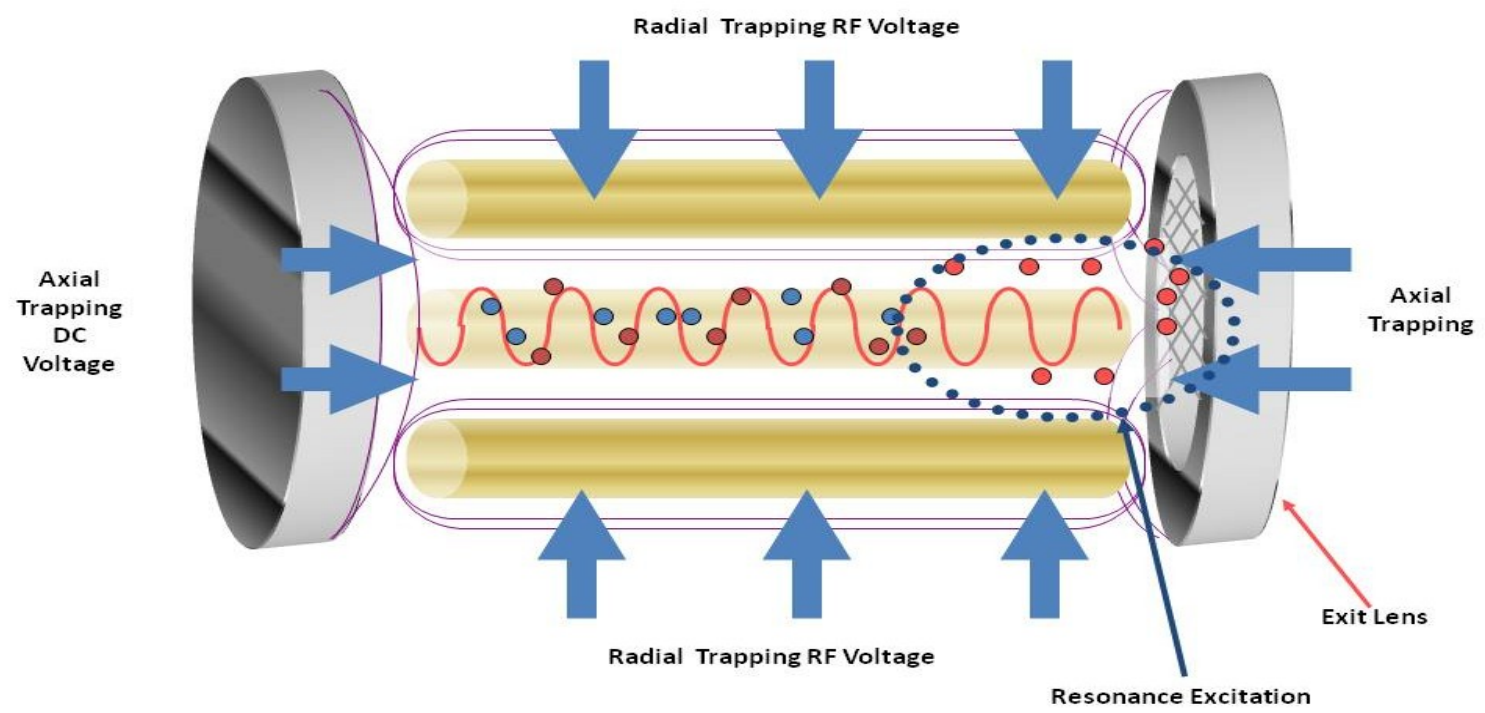

Figure 1.12: The magnetic fields imposed upon an ion in a quadrupole LIT [44] 
MS detectors convert the electrode neutralization of an ion into a quantifiable electrical signal. Two detectors were used in this dissertation: a microchannel plate (MCP) detector in the QStar XL mass spectrometer and a channel electron multiplier (CEM) in the 4000 Qtrap instrument. MCP's are a collection of small channel electron multipliers configured into two chevrons (Figure 1.13). The impingement of an ion at the first chevron leads to electron emission. As the electrons travel through the channel, each additional collision with the electrode wall leads to further electron release. The electrons migrate along the electric field into the second Chevron that is rotated 180 degrees and electron emission is further amplified. The electron cascade is collected at an anode and converted into a measurable electrical current to provide an ion intensity that is proportional to ion impingement [51]. MCP detectors are necessary following a TOF mass analyzer due to the spatial differences of the incoming ions. A CEM detector is more sensitive and relies on the same principles. Ion impingement at a conical electrode leads to the emission of up to three electrons. An electric field is applied axially along the cone to cause further electron emission and the electrons are similarly collected at an anode and converted to a digital output [52]. 


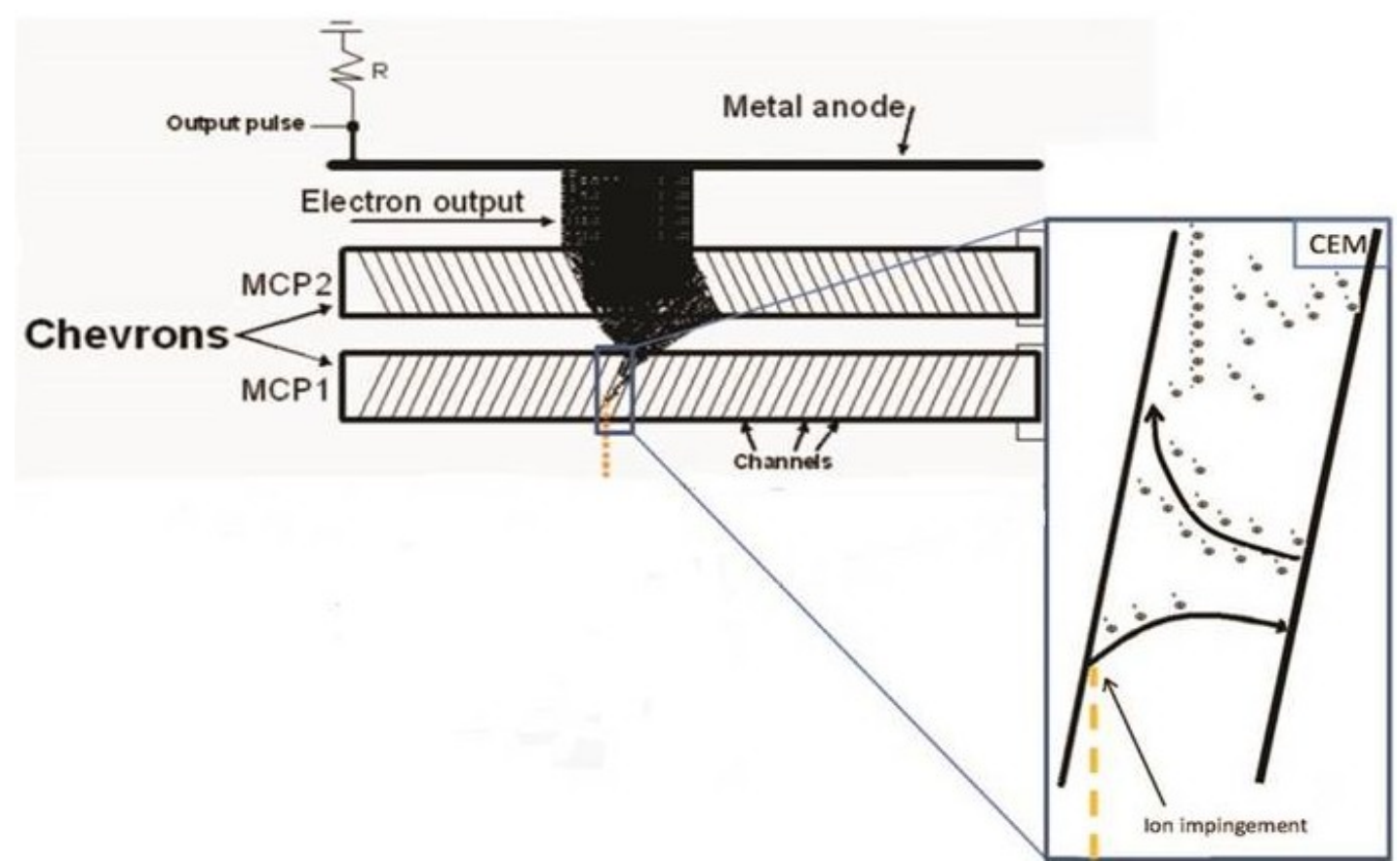

Figure 1.13: Ion impingement at an MCP detector leads electron emission and electrical current output [48]

ESI-MS can be coupled to liquid chromatography (LC) on the front end prior to ionization. This allows for sample separation to concentrate analytes based on their molecular properties. This is particularly important for non-abundant species that would otherwise have their intensities masked by abundant ions. Analytes are passed over a solid phase that exclusively interacts with the analyte. The mobile phase composition is altered leading to analyte elution from the solid phase and migration towards the ionization source. Numerous LC options are available such as: strong cation exchange (SCX), strong anion exchange (SAX) and normal-phase. SCX chromatography uses sulfonate groups linked to a solid support to preferentially bind cationic species. Oppositely, SAX beads have the sulfonate groups replaced with quaternary ammonium groups which interact with anionic compounds. Analyte elution depends on the salinity or $\mathrm{pH}$ of the mobile phase. Normal phase chromatography uses a hydrophilic silica support that binds highly polar substances and eluted with polar mobile phase solvents. In this 
dissertation, reversed-phase (RP) LC was connected online to the front end of the mass spectrometer because it is favourable for the analysis of small molecules and biomacromolecules. Hydrophilic analytes bind to a column containing a solid support with hydrophobic C18 groups. Analytes elute from the column when the organic composition of the mobile phase has been substantially elevated to out-compete the analyte for $\mathrm{C} 18$ binding.

Of note, coupled LC-MS has advanced two applicable areas of research: the characterization of small molecule pharmaceuticals and proteomics. Pharmaceuticals can be monitored by LC-MS in the pre-clinical development phase for the purposes of stability and molecular properties testing, as discussed in greater detail in Chapter 3.

Proteins are biomolecule polymers consisting of amino acid monomers. They can be analyzed by LC-MS using two approaches: top-down or bottom-up [41]. In top-down strategies, proteins remain intact and their molecular weight is evaluated using the envelope of ion intensities resulting from various charged states. Bottom-up proteomic techniques involve the use of a protease enzyme to cleave the peptide backbone to produce peptides of varying lengths. Proteins are often separated prior to digestion using sodium dodecyl sulfate polyacrylamide gel electrophoresis (SDS-PAGE) to reduce sample complexity. In peptide fingerprinting, the masses of the detected peptides are compared to ion databases and provide the most probable protein identity. More thoroughly, de novo sequencing of peptides is accomplished by fragmenting the peptides. Depending on the location of the localized charge and the peptide backbone cleavage site, series of $y$-ions and b-ions are observed that represent sequential neutral amino acid losses (Figure 1.14) [53]. This is achieved through independent data acquisition (IDA) 
that monitors the 1 second full mass scan in every duty cycle and selects the four most intense multiply charged ions for fragmentation and 3 second product ion scans. Once a peptide ion has been selected for fragmentation, it is placed on an exclusion list for 90 seconds to maximize the sequencing of less abundant peptides.

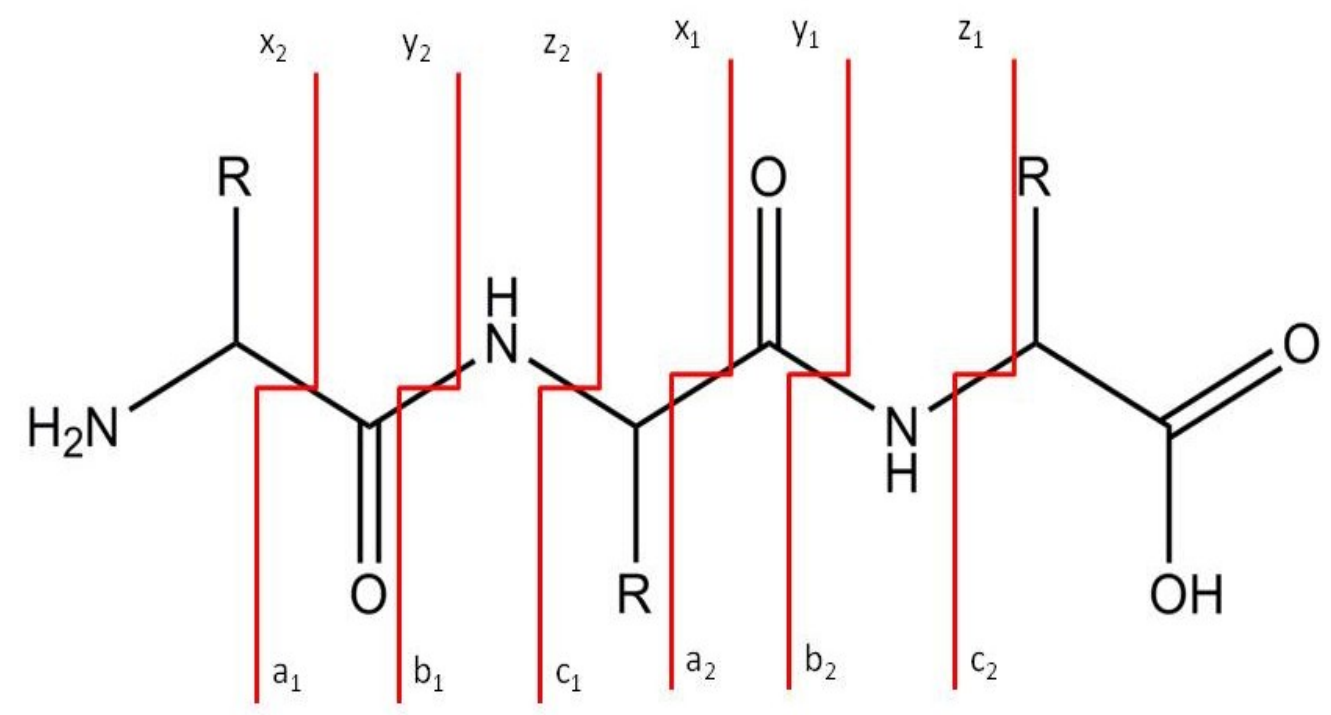

Figure 1.14: The CID fragmentation patterns of peptides. Adapted from [53]

Trypsin is the protease that is typically used in bottom-up proteomics because it cleaves peptides bonds at the carboxyl side of arginine and lysine residues, unless either is followed by a proline residue. This is favourable for ESI-MS for multiple reasons. Firstly, the tryptic digestion produces peptides of suitable lengths for MS analysis in a predictable manner to facilitate comparisons to spectral databases with higher protein sequence coverage. Secondly, the peptides terminate with an arginine or lysine residue that possesses side-chains with high proton affinities in relation to typical reversed-phase solvents (Table 1.1). This ensures that all peptides will ionize with multiple charges so that all fragments are detectable for sequencing. The proton affinities for pyrrole and 
butyrolactone are included in Table 1.1 because they are common VSe structural scaffolds. This indicates that VSes are also detectable by positive-mode LC-MS since their proton affinities are higher than water and acetonitrile.

Table 1.1: Proton affinities of applicable compounds and solvents [54]

\begin{tabular}{cc}
\hline Compound & $\begin{array}{c}\text { Proton Affinity } \\
(\mathrm{kJ} / \mathrm{mol})\end{array}$ \\
\hline Arginine & 1051 \\
Lysine & 996 \\
Histidine & 988 \\
Dimethylsulfoxide* & 885 \\
Pyrrole* & 875 \\
Butyrolactone* & 840 \\
Acetonitrile & 779 \\
Water & 691 \\
\hline
\end{tabular}

* data obtained from [55]

The peptide product ion scans are compared to a database, such as Mascot, which calculates the probability of the observed amino acid sequence being a random event. The protein identity match with the lowest possibility is given the highest score. Bottom-up proteomic approaches are not limited to protein identification applications, but also for observing post-translational modifications and performing protein quantitation that is useful in diagnostic biomarker identification.

As herein described, LC-MS was a suitable approach to evaluate the physiochemical and proteomic properties of VSes with hopes of improving the stability and mechanistic knowledge surrounding this novel class of small molecules. 


\section{Chapter: Sample Preparation and MS Methods}

A general description of the materials and methods commonly used in this work is described below. Subsequent chapters designate the experiment-specific alterations made to these protocols in greater detail.

\subsection{Protein digestion}

Proteins were received in two formats in preparation for bottom-up proteomic analysis: solubilized or isolated as an electrophoresis gel band. Solubilized proteins were denatured by adding $1 \mathrm{M}$ dithiothreitol (DTT) (Thermo Scientific) to a concentration of 5 $\mathrm{mM}$ for $1 \mathrm{hr}$ at $56^{\circ} \mathrm{C}$ to reduce the disulfide bridges. This disrupts the protein's tertiary structure and makes the protease substrate bonds more accessible for cleavage (Figure 2.1 A). Once the sample had cooled to room temperature, $100 \mathrm{mM}$ iodoacetamide (Sigma Life Science) was added to a concentration of $10 \mathrm{mM}$ for $1 \mathrm{hr}$ in the dark. This alkylates the cysteinyl thiol groups with carbamidomethyl groups to prevent the reformation of disulfide bridges (Figure $2.1 \mathrm{~B}$ ). Five percent of the protein's mass equivalent in sequencing-grade trypsin (Promega) was added to the solution and allowed to incubate overnight (12-16 hrs) on a shaker at $37^{\circ} \mathrm{C}$. Digested peptide samples were stored at $-20^{\circ} \mathrm{C}$ until LC-MS analysis. 


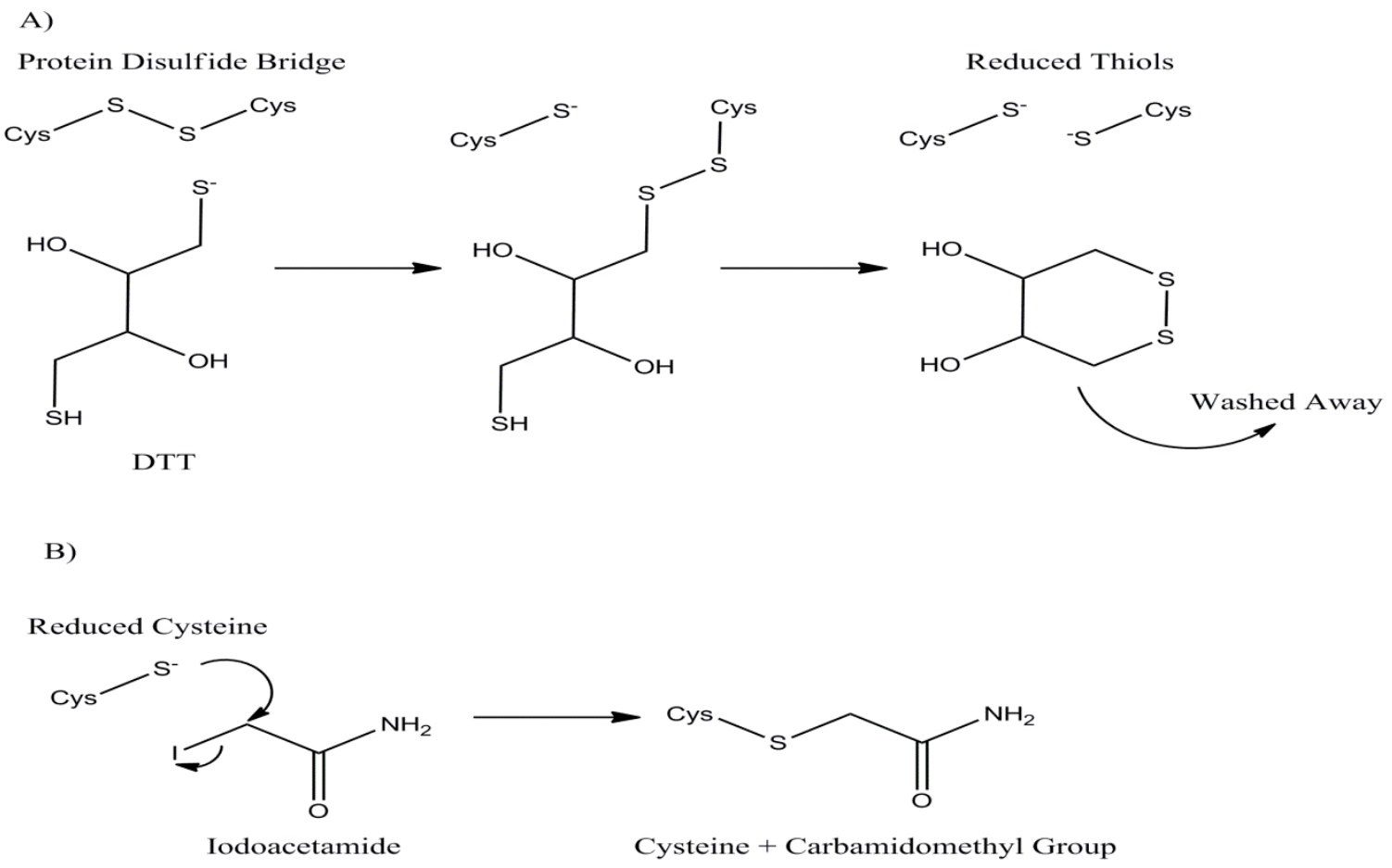

Figure 2.1: The reduction and alkylation of disulfide bonds to prepare proteins for tryptic digestion

Proteins that were isolated in gel bands underwent a more extensive digestion protocol (Figure 2.2) as adapted from Wilm et al. [57] . Measures were taken to minimize keratin contamination throughout the duration of the digest. A razor blade was used to excise and cut gel bands into pieces no greater than $1 \mathrm{~mm}^{2}$ in size. The gel fragments were suspended in $100 \mu \mathrm{L}$ of $50 \mathrm{mM}$ ammonium bicarbonate (ABC) (MP Biomedicals) and washed for 20 mins. The fragments were centrifuged at $1000 \mathrm{rpm}$ (Sorvall Legend Micro $21 \mathrm{R}$ centrifuge, Thermo Scientific) for $1 \mathrm{~min}$ and the $\mathrm{ABC}$ solution was removed. The fragments were completely destained and dehydrated with repeated 20 min washes of $50 \%$ acetonitrile $(\mathrm{ACN})$ in $50 \mathrm{mM} \mathrm{ABC}$ solution. The fragments were completely dried by a SC100 SpeedVac concentrator (Savant) equipped with a RVT400 refrigerated vapour trap (Thermo Savant) and treated with $100 \mu \mathrm{L}$ of $10 \mathrm{mM}$ DTT solution for 15 
mins at $56^{\circ} \mathrm{C}$. The DTT solution was replaced with $100 \mu \mathrm{L}$ of $100 \mathrm{mM}$ iodoacetamide for 15 mins in the dark. The iodoacetamide solution was removed and the gel fragments were completely dried once more. The $\mathrm{ABC}$ and $50 \% \mathrm{ACN}$ washing steps were repeated to remove remnant DTT and iodoacetamide salts. $20 \mu \mathrm{g}$ of sequencing-grade trypsin was solubilized in $400 \mu \mathrm{L}$ of $50 \mathrm{mM} \mathrm{ABC}$ solution and $50 \mu \mathrm{L}$ was added to the dried gel fragments. Protein digestion was performed overnight $(12-16 \mathrm{hr})$ on a shaker at $37^{\circ} \mathrm{C} .25$ $\mathrm{mM} \mathrm{ABC}$ solution was added as needed to keep the gel fragments completely suspended in solution. The resultant peptide solution was collected following a brief centrifugation step. The gel fragments were washed with $25 \mathrm{mM}$ ABC solution for 20 mins and the solution was combined with the peptide solution from the previous step. The gel fragments were washed with $50 \% \mathrm{ACN}$ in $5 \%$ aqueous formic acid (Sigma Aldrich) solution for 20 mins and the solution was combined with the peptide solution from the previous steps. Peptide samples were completely dried and resuspended in $20 \mu \mathrm{L}$ of $0.1 \%$ aqueous formic acid (FAw), sonicated for 10 mins in a water bath (Mettler Electronics Corp.) and stored at $-20^{\circ} \mathrm{C}$ until LC-MS analysis. 

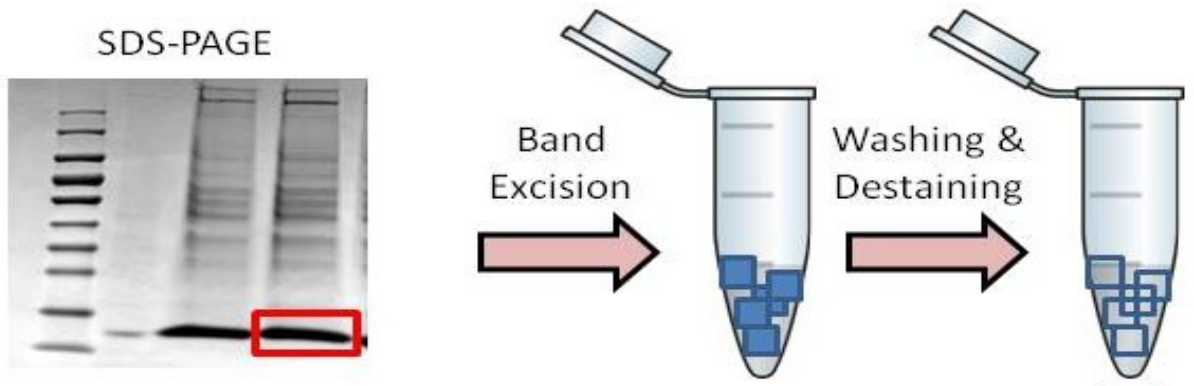
Protein ID by LC-MS/MS
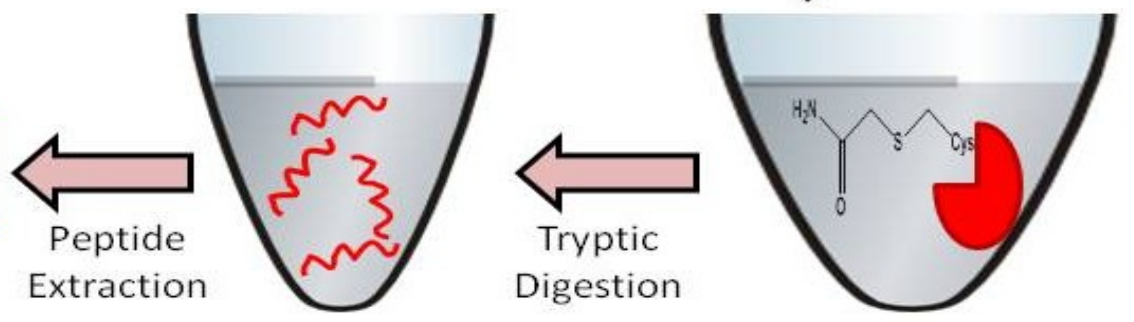

Figure 2.2: General procedure for the in-gel tryptic digestion of proteins

Prior to analysis, peptide samples were desalted by one of two RP-C18 solidphase extraction techniques: zip-tips (Millipore Corp) or Omix-tips (Varian) that possess binding capacities of $2 \mu \mathrm{g}$ and $80 \mu \mathrm{g}$ (respectively). Peptides from gel bands were typically desalted using the former while solubilized peptides with the latter. The tip was washed with three volumes of Solution A ( $70 \% \mathrm{ACN}$ in $0.1 \% \mathrm{FAw})$ and three times with Solution B $(0.1 \% \mathrm{FAw})$. The analyte was repeatedly bound to the stationary phase, washed five times with Solution B and eluted with five volumes of Solution A.

\subsection{Spin filtering}

The majority of VSe assays require the separation of the small molecules from large biomolecules that would cause high pressure issues during LC-MS analysis. This 
was achieved by passing VSe samples through spin filters (Millipore Corp.) with a molecular weight cutoff of $3 \mathrm{kDa}$ (Figure 2.3).

$500 \mu \mathrm{L}$ of VSe sample was passed through the spin filter into a collection tube by centrifugation at $13000 \mathrm{rpm}$ for 30 mins. VSe and metabolite samples were stored frozen at $-20^{\circ} \mathrm{C}$ until LC-MS analysis. The macromolecules that were trapped in the filter, such as proteins, were collected by adding $100 \mu \mathrm{L}$ of resolubilization buffer to an inverted spin filter. Collection of the macromolecules was accomplished by spinning the filters at 2000 rpm for 5 mins into a new collection tube [58].
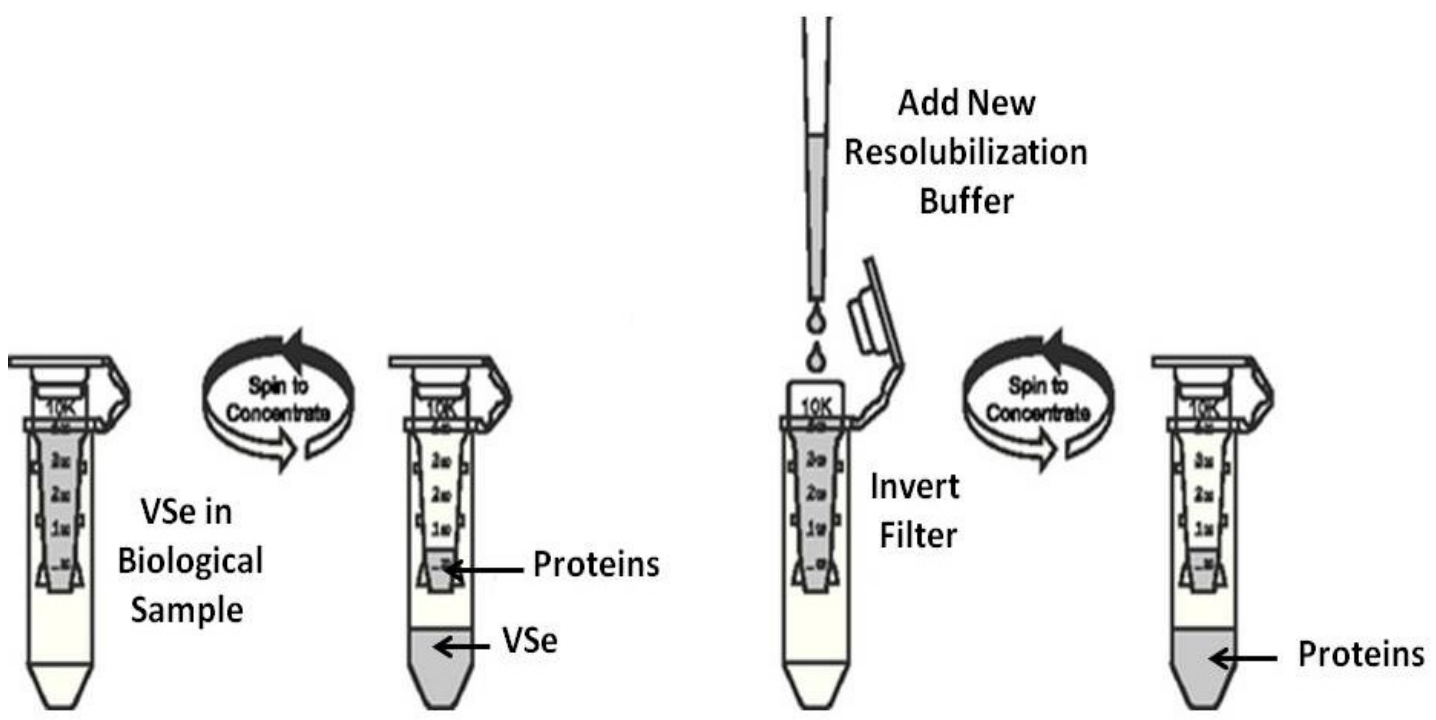

Figure 2.3: Spin filtering to isolate VSes from complex biological samples. Adapted from [58]

\subsection{Direct infusion MS}

MS analysis of VSe stock solutions was performed direct infusion that lacks the front-end HPLC separation. These experiments were performed on the hybrid triple quadrupole time-of-flight (QqTOF) QStar XL mass spectrometer (AB Sciex). The TOF mass analyzer was externally calibrated by obtaining the mass spectrum of a CsI solution 
containing a standard peptide. $5 \mu \mathrm{L}$ of the calibration solution was loaded to the tip of a NanoESI spray capillary (Thermo Scientific). The tip was cracked upon contact with the charged curtain plate. Direct infusion experiments used an ionspray voltage of $1500 \mathrm{~V}$, a declustering potential of $30 \mathrm{~V}$, a focusing potential of $120 \mathrm{~V}$, a second declustering potential of $15 \mathrm{~V}$, a CID collision gas setting of 2 and an ion gas source setting of 1 . Once the TOF mass analyzer was externally calibrated to within 5 ppm mass accuracy, VSe samples were analyzed by direct infusion under the same parameters. Accurate mass experiments using a full mass scan were used to identify VSe powders from the library of analogues and develop MRM methods. VSes were also subjected to product ion scans following direct infusion when the CID collision gas setting was increased to 5 and the optimal collision energy was adjusted accordingly.

\subsection{LC-MS}

The majority of experiments required the separation of VSes, metabolites or peptides from a complex biological sample. To enhance the signal of analyte ions that would otherwise be masked by direct infusion, the complex samples were separated by an Ultimate 3000 pump and autosampler RP-HPLC (Dionex) prior to eluate ionization. The selected RP-LC solvents were $0.1 \%$ FAw as solvent A and $0.1 \%$ formic acid in ACN as

solvent B (Fischer Scientific). The $200 \mu \mathrm{m}$ inner diameter (ID) silica columns (Polymicro Technologies) were fritted by briefly dipping one end into a mixture of $88 \mu \mathrm{L}$ Kasil1624 potassium silicate (National Silicates) and $16 \mu \mathrm{L}$ formamide catalyst (Promega). Frits were dried overnight and the columns were packed in-house with a slurry of MAGIC C18 
RP-beads (5 $\mu \mathrm{m}$ diameter) (Michrom Bioresources Inc.) in acetone using a high-pressure vessel to a length of $5 \mathrm{~cm} \mathrm{[59].}$

For a typical LC-MS analysis, $20 \mu \mathrm{L}$ of sample was transferred to an amber HPLC autosampler vial (Fisher Scientific) with a conical spring insert (Canadian Life Science). The sample was loaded onto the RP column using $2 \%$ solvent B with a low flow rate to accommodate the high pressures associated with the switching valve in the load position (Figure $2.4 \mathrm{~A}$ ). In this position, the back-pressure caused by the reduction in ID from the second T-connector to the ESI emitter causes the unbound sample salt to predominantly flow through the second waste line. The first waste line is closed to force the entirety of the sample through the column for maximal analyte binding. Once the sample was loaded onto the column and several minutes of desalting had passed, the flow rate and thus the pressure were reduced to 0 and the switching valve changed to the inject position. In this position, higher flow rates are permitted since the smaller ID waste line from the first T-connector is open which allows some solvent to flow to waste while the remainder enters the column. Once through the column, the second waste line is closed to force all eluate to the ESI emitter and maximize analyte ion signal (Figure 2.4 B).

When using the QStar XL instrument, the HPLC eluate was coupled to an ionspray source with a $15 \mu \mathrm{m}$ ID picotip emitter (New Objective) that was also packed 5 cm with $5 \mu \mathrm{m}$ diameter RP-C18 beads. The hybrid triple quadrupole linear ion trap 4000 Qtrap mass spectrometer (AB Sciex) was equipped with a Turbo V ion source (AB Sciex) that did not feature packed RP beads. 
A) Load Position

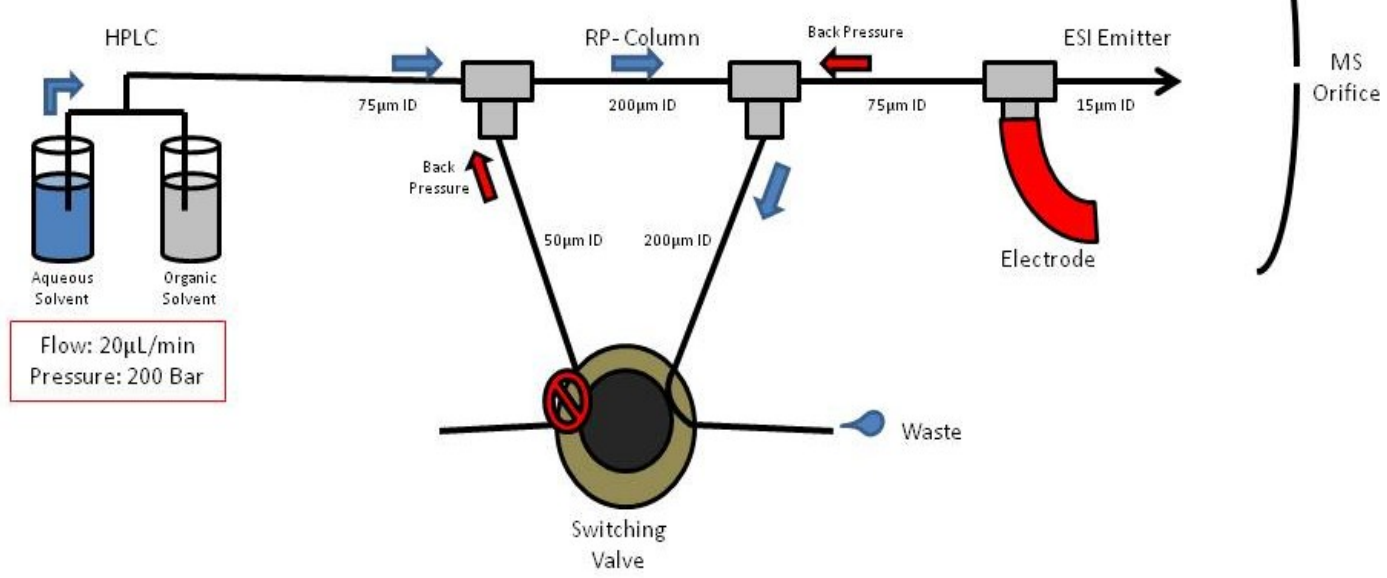

B) Inject Position

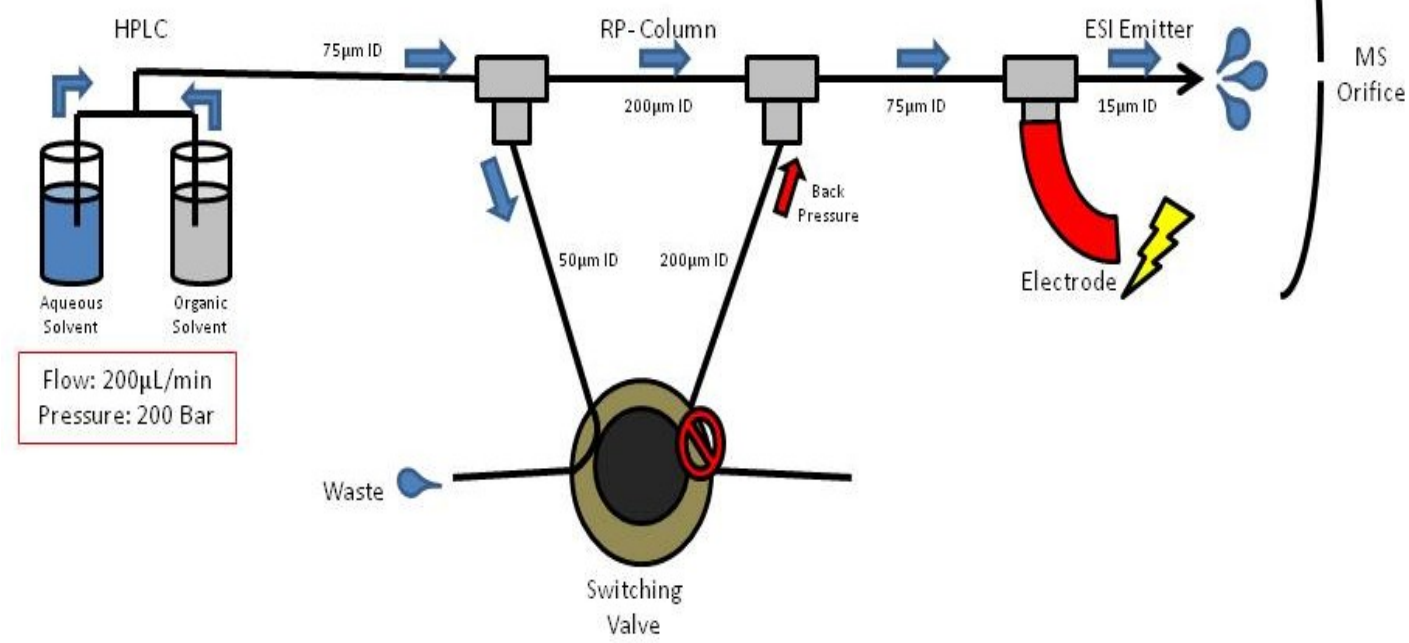

Figure 2.4: Schematic diagram of the LC-MS switching valve set to the load (A) and inject (B) positions

Different HPLC gradients were required to obtain VSe chromatograms with optimal resolution. Two gradients were commonly used on the QStar XL instrument. The "30 min proteomics" method (Figure 2.5) was used to analyze peptide samples and performed 164 cycles of a full mass scan from 400-1500 Th and four product ion scans of the most intense multiply $(2+, 3+, 4+)$ charged ions. Optimal product ion collision 
energies for each peptide selected for fragmentation were computationally calculated from the $\mathrm{m} / \mathrm{z}$ of the peptide. The majority of peptides eluted at low percentages of organic solvent, thus peptide co-elution was minimized by slowly increasing the mobile phase composition to $50 \% \mathrm{ACN}$. The method then quickly increased to $100 \% \mathrm{ACN}$ to rid the column of large, non-polar biomolecules. The method was concluded with a system reequilibration to $2 \% \mathrm{ACN}$ to maximize peptide binding in the next run.

Peptide LC-MS data was analyzed using MASCOT database searches for protein identification. In general, the NCBI protein database was searched with cysteinyl carbamidomethyl fixed modification, methionine oxidation variable modification, with a peptide mass tolerance of $100 \mathrm{ppm}$ and an MS/MS tolerance of $0.3 \mathrm{Da}$. The database searched for $[\mathrm{M}+2]^{+2},[\mathrm{M}+3]^{+3}$, and $[\mathrm{M}+4]^{+4}$ charged peptides with up to one missed tryptic cleavage. MASCOT scores were formatted using standard scoring with a peptide score cutoff of $>20$ and required bold red labelling.

The "30 min sensitizer" gradient used to analyze VSe QC and metabolite samples linearly increases from $2 \% \mathrm{ACN}$ to $100 \% \mathrm{ACN}$ followed by a system re-equilibration (Figure 2.6). The "30 min sensitizer" method performed 1805 full mass scans of a 103800 Th window. These two methods used the same parameters as those described for the direct infusion experiments but the ionspray voltage was increased to $3700 \mathrm{~V}$ and the ion source gas was set to 0 .

Two gradients were often used on the 4000 Qtrap instrument to separate sets of VSe analogues and their metabolites. "VSe Gradient A" was employed to separate VSes that readily bound to the column (Figure 2.7). Given that VSes typically eluted when the ACN composition of the mobile phase reach $50-80 \%$, the sharper gradient steepness from 
$50-80 \% \mathrm{ACN}$ was implemented to improve chromatogram resolution, and hence improve AUP quantitation accuracy. "VSe gradient B" was used for VSes with increased polarity, and had difficulty binding to the RP column. This gradient featured a shorter sample loading time to ensure that unbound VSes were still ionized instead of being washed away. The majority of these VSes eluted immediately from the column, therefore the increased initial steepness of the gradient was necessary to improve chromatogram resolution (Figure 2.8). These two methods used an ionspray voltage of $5000 \mathrm{~V}$, a declustering potential of $25 \mathrm{~V}$, entrance and exit potentials of $10 \mathrm{~V}$, and ion source and curtain gas settings of 20. 652 cycles of an enhanced mass scan (EMS) and multiple MRM scans were performed under these methods. Changing from a 30 mins gradient to a 15 mins gradient increased assay throughput to accommodate the greater quantity of samples associated with MRM analysis.

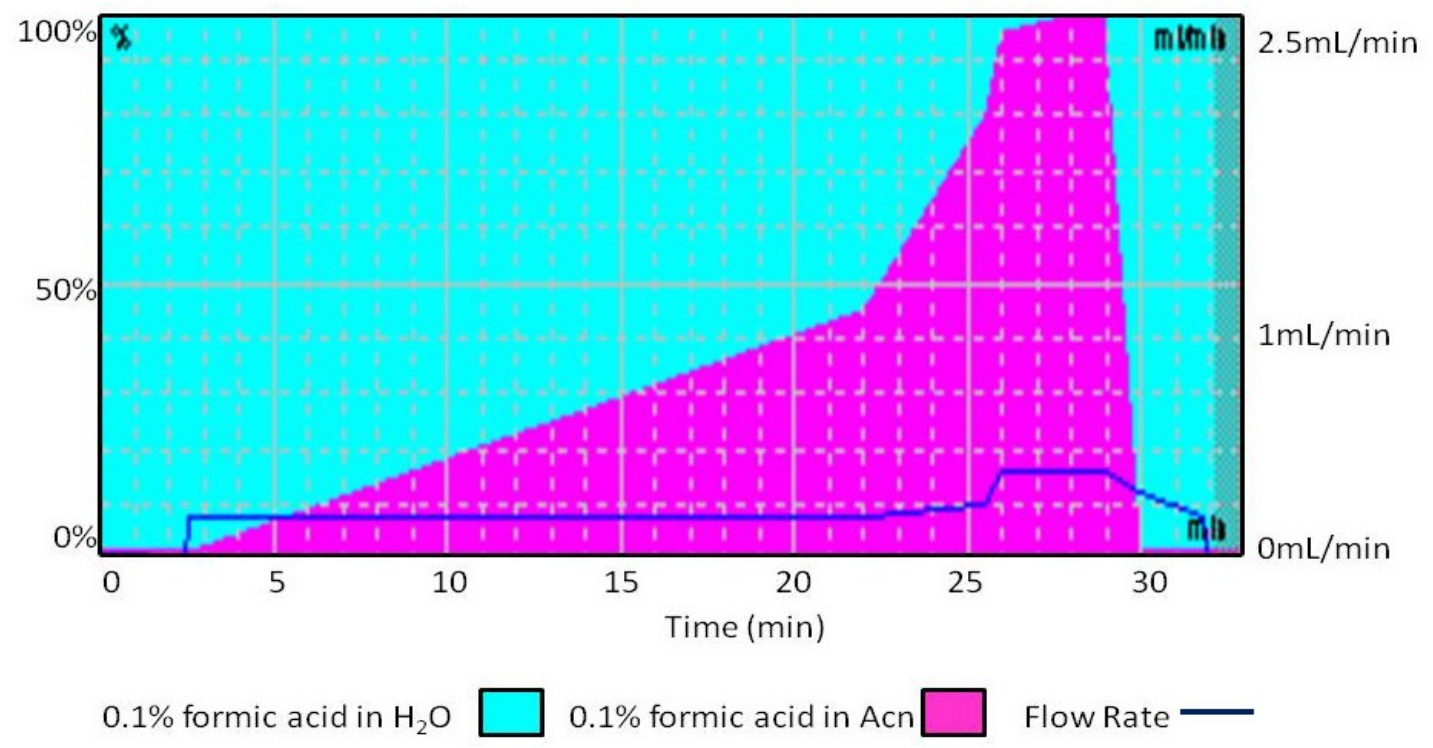

Figure 2.5: 30 min proteomics gradient for the analysis of peptides on the QStar XL 


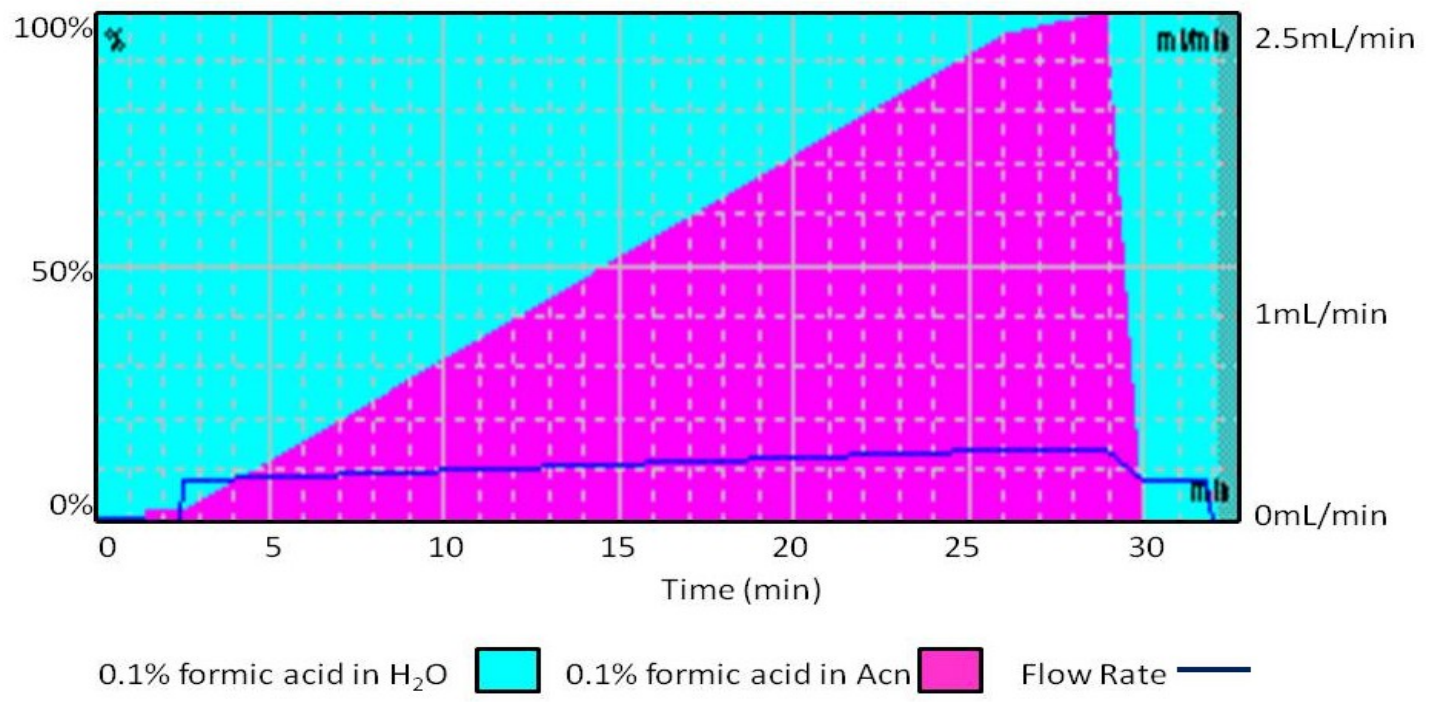

Figure 2.6: 30 min sensitizer gradient for the analysis of VSe stocks and metabolites on the QStar XL

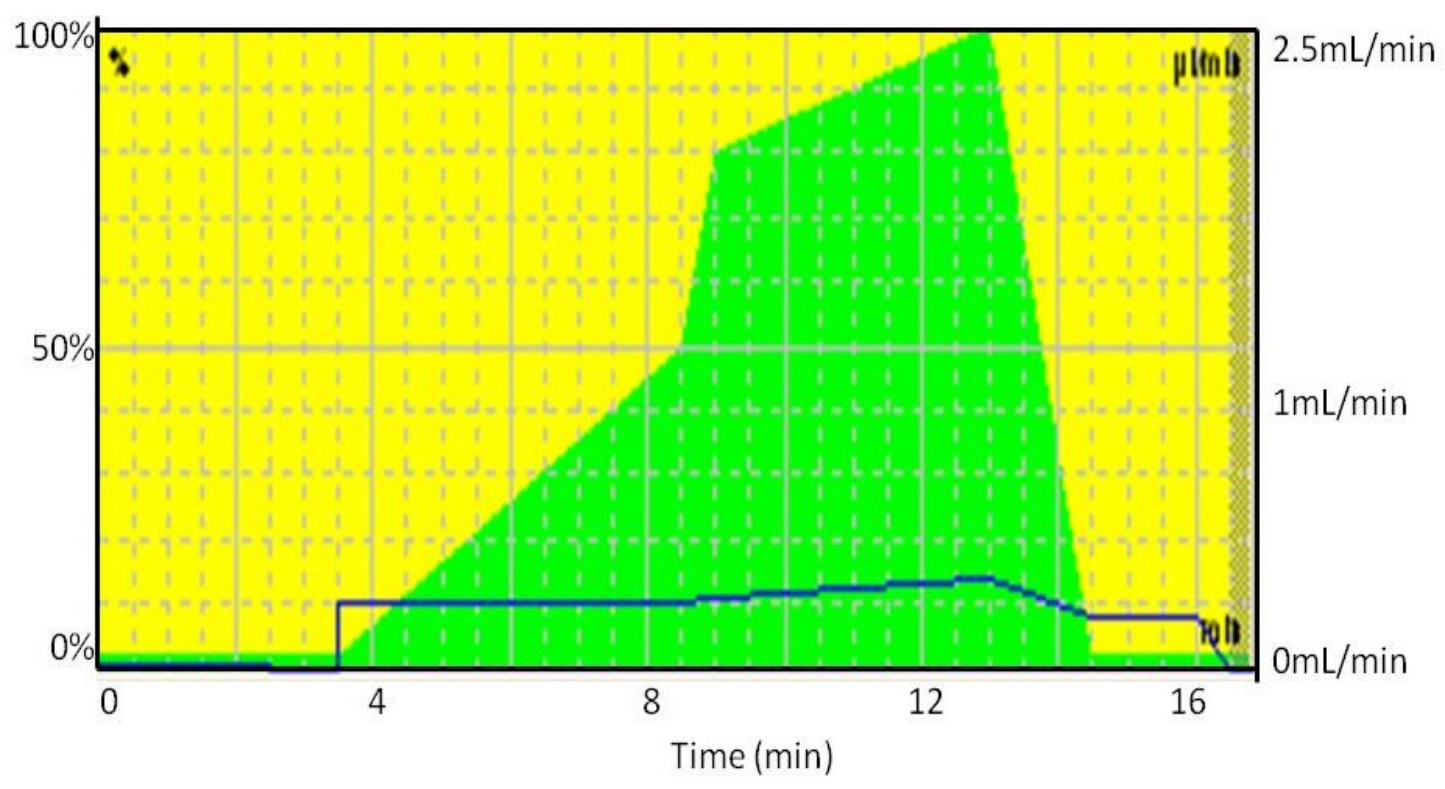

$0.1 \%$ formic acid in $\mathrm{H}_{2} \mathrm{O} \square$ 0.1\% formic acid in Acn $\square$ Flow Rate -

Figure 2.7: VSe Gradient A for the analysis of VSe stocks and metabolites on the 4000 Qtrap MS 


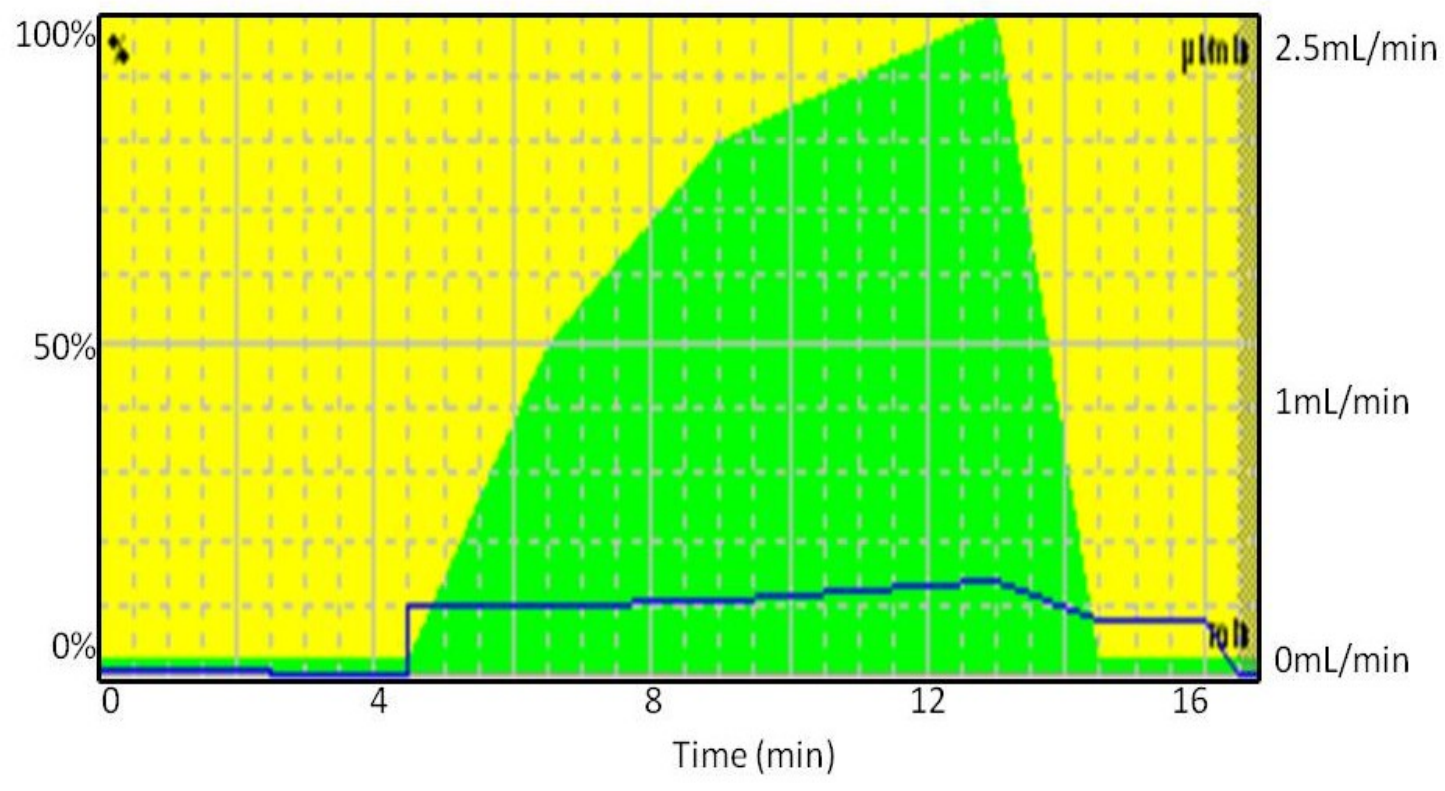

$0.1 \%$ formic acid in $\mathrm{H}_{2} \mathrm{O} \square 0.1 \%$ formic acid in $\mathrm{Acn} \square$ Flow Rate $\square$

Figure 2.8: VSe Gradient B for the analysis of VSe stocks and metabolites on the 4000 Qtrap MS 


\section{Chapter: VSe Quality Control Analysis}

\subsection{Quality Assurance and LC-MS}

Small molecule pharmaceuticals endure a rigorous clinical testing process prior to market approval. One component of drug manufacturing that could potentially limit clinical effectiveness is the ability to produce the final product in large quantities in a reproducible manner. The study of pharmaceutical quality control (QC) involves the required measures to ensure the consistent identity and quantity of drug preparations. A critical aspect of the VSe pre-clinical research phase is a guarantee that the prescribed amount of the small molecule is actually delivered to the attenuated virus. This eliminates the drug preparation as a possible source of error when unexpected results arise during pre-clinical development.

There are numerous considerations surrounding the preparation of small organic molecules including: solvent choice, temperature, $\mathrm{pH}$, photosensitivity, humidity, purity and oxidation. Monitoring each of these variables for pharmaceutical storage, dilution and administration creates a demand for dependable analytical chemistry techniques. LCMS is widely considered the primary detection method in drug research and development (R\&D), particularly in QC. The two dimensions of analyte separation (polarity and $\mathrm{m} / \mathrm{z}$ ) produce a unique signature for each analyte to reduce the rate of false-positive results; a concern with using either technique individually. Additional advantages LC-MS provides relative to other analytical techniques include it's high throughput and robustness [60]. This is particularly valuable in large production facilities where a large number of samples need to be identified and quantified with the added pressure of potentially delaying the product's market release. 
Despite being considered the golden standard in small molecule QC, LC-MS still has room for improvement. Upgrades to LC-MS instrumentation continually instilling more confidence in QC results [60]. Significant improvements in throughput, elution profile resolution, quantitation reproducibility and sensitivity relative to the traditional HPLC can be attributed to the development of ultra performance liquid chromatography (UPLC). UPLC applies an improved pump system and utilizes a smaller $2 \mu \mathrm{m}$ RP-bead size in comparison to the $5 \mu \mathrm{m}$ beads used in HPLC. This permits the use of higher flow rates that are not hindered by excessive pressures to improve through-put. The smaller particle size allows for increased column binding capacity and chromatogram resolution. The enhanced binding capacity also yields a 2-3 fold enhancement in small molecule sensitivity in comparison to HPLC [61]. UPLC (10-100 $\mathrm{nL} / \mathrm{min})$ can be equipped with line splitters that permit for higher pressures that translate into improved sensitivity during NanoESI-MS in comparison to standard electrospray sources associated with HPLC $(>1 \mu \mathrm{L} / \mathrm{min})$ [62]. Significant instrumentation improvements have been observed in TOF technology that provide 2 ppm mass accuracy that extends over a wider mass range [60]. Advancing ion trap technology with enhanced dynamic and linear ranges are allowing for QC results to confidently draw conclusions about small molecule storage solvent choices [63].

Comparisons made between small molecule stability factors or different batches of drug manufacture require a dependable method of quantitation. Extensive method validation is necessary to ensure that fluctuations in drug quantity can solely be attributed to the stability or preparation batch in question. Method validation properties including limit of blank (LOB), limit of detection (LOD) and limit of quantitation (LOQ) are 
determined. LOB is the maximum quantity of ion intensity produced from an LC-MS injection in which the analyte is absent. This provides a threshold for the LOD; the minimum concentration of analyte that can be distinguished from the LOB and is defined as the LOD $+1.645(\mathrm{SD}$ of $\mathrm{LOB})$. Knowledge of the LOD is important because if no signal is observed in the analysis of drug preparation, it can be stated with confidence that the sample contained a lower concentration than the LOD. The LOQ is the concentration at which the linear range of quantitation begins, thus indicating concentrations greater than the LOQ are suitable for quantitation testing. Determination of these parameters for every analyte under QC testing are characteristics of a thoroughly validated method [64].

QC quantitation methods are often supplemented with the quantitation of an internal standard. Internal standards are implemented to ensure that the instrument is operating as expected to eliminate instrument malfunctions as a source of error when the analyte produces unexpected ion intensity. These include small variations in the injection volume and "matrix effects" during the MS ionization process. Matrix effect is an allencompassing term used to describe abnormal analyte behavior. A common example is ion enhancement or suppression during the ionization process caused by the co-elution of other ions. These include but are not limited to: degradation products of the analyte, salts and their analyte adducts and common solvents such as DMSO and phosphate-buffered saline (PBS) [65]. The selection of an internal standard is another important step to the QC method development process which provides another degree of confidence in the method.

A final aspect of method development is the type of quantitation to be used: relative and absolute. The difference lies in the use of a standard curve that correlates 
analyte concentration to the ion intensity in absolute quantitation. Relative quantitation requires less method development as it compares the ion intensity of an analyte across multiple samples in relation to itself, but is deemed inferior [66]. In this chapter, the results of preliminary QC experiments that utilize both types of quantitation are presented, discussed and related to unexpected pre-clinical VSe results.

\subsection{Caffeine internal standard}

Ideally, internal standards are stable-isotope labeled forms of VSes however, their synthesis is time-consuming and expensive. As a result, selecting an internal standard that behaves similarly to VSes is the next ideal option [65] that must meet certain criteria. Firstly, the internal standard should be stable in the conditions to which it will be subjected. In this case, the molecule will be stable in storage at ambient temperature, in the acidic mobile phase of the HPLC and in the gas-phase during ionization. The standard cannot be isobaric with the analyte of interest while still able to be separated by RPHPLC. Caffeine was chosen as an internal standard for VSe quantitation as it meets all of these requirements with the additional advantages of being readily available, inexpensive and widely used.

A $5 \mu \mathrm{g} / \mathrm{mL}$ aqueous solution of caffeine (Analytical Solutions) was diluted in $\mathrm{ddH}_{2} \mathrm{O}$ and spiked into a total HPLC injection volume of $20 \mu \mathrm{L}$ at varying volumes and concentrations ( 1 or $2 \mu \mathrm{L}$ of $5-0.1 \mu \mathrm{g} / \mathrm{mL}$ ). A full mass scan by direct infusion on the QStar XL instrument confirmed the presence of caffeine with an $[\mathrm{M}+\mathrm{H}]^{+1}$ ion at 195.0925 Th that is comparable to the expected ion of 195.0882 Th (Figure 3.1). Caffeine spikes were analyzed by a TOF full mass scan following RP-HPLC separation and the 
area-under-the-peak (AUP) of the 195 Th XIC was calculated by MultiQuant software. The "30 min sensitizer" LC-MS method was used for QC analysis (Chapter 2). A $250 \mathrm{ng}$ spike of caffeine proved to be excessive because it caused chromatogram tailing and detector saturation indicated by the $80 \%$ relative signal intensity produced by the $196 \mathrm{Th}$ ion (Figure 3.2). Therefore, this amount of caffeine was beyond the dynamic range of the detector. A $5 \mathrm{ng}$ spike (in $1 \mu \mathrm{L}$ ) of $5 \mu \mathrm{g} / \mathrm{mL}$ caffeine stock was determined to be optimal because it did not overload the detector and it avoided the error introduced with further dilution of the commercial stock. Additionally, the spike volume was small in comparison to the $20 \mu \mathrm{L}$ HPLC injection which gave more volume priority to the sample of interest (Figure 3.3).

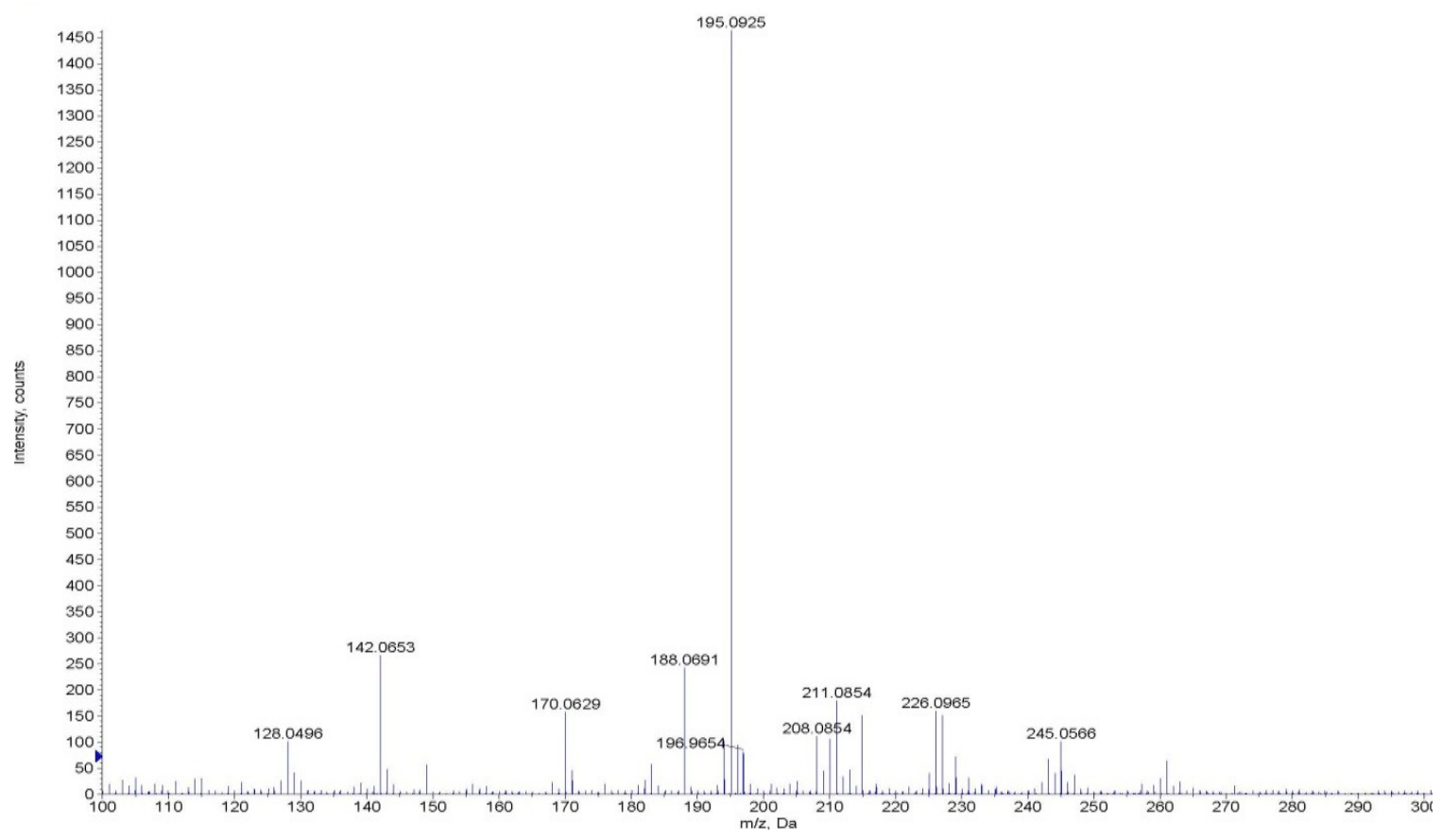

Figure 3.1: Direct infusion accurate mass scan of caffeine. 

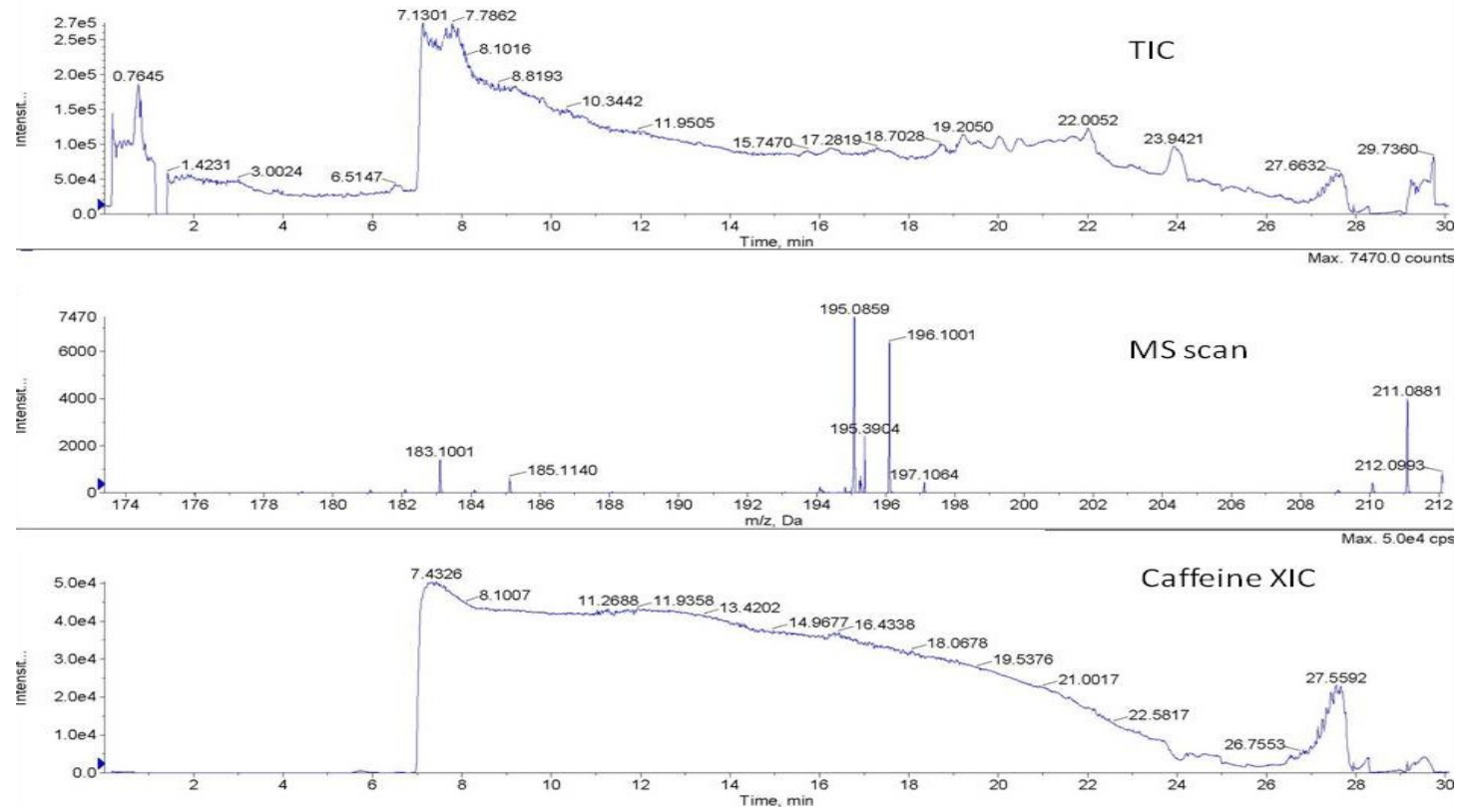

Figure 3.2: LC-MS of a $250 \mathrm{ng}$ spike of caffeine.
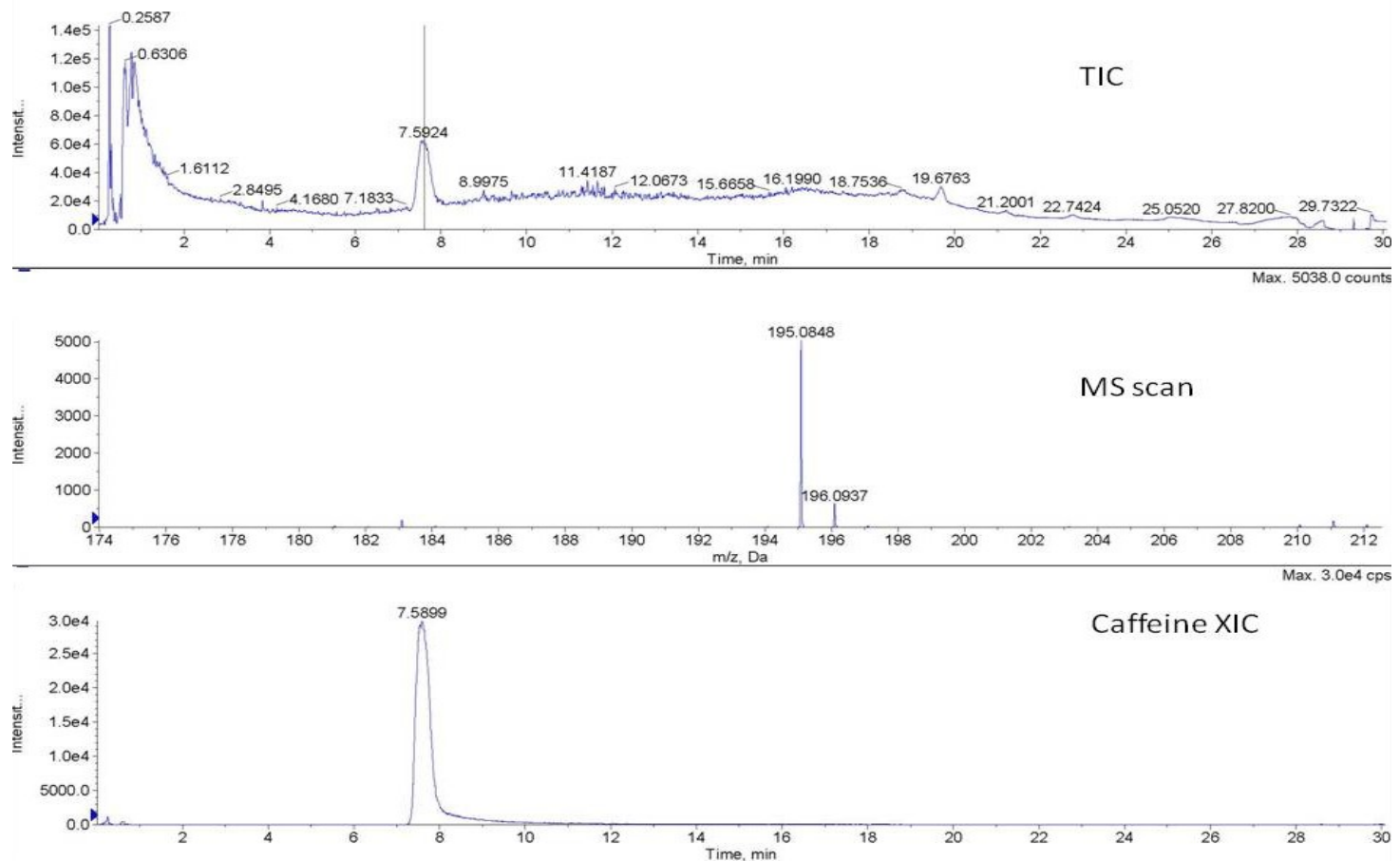

Figure 3.3: LC-MS of a $5 \mathrm{ng}$ spike of caffeine. 
The optimal spike volume and concentration provided a reproducible signal on an equimolar run-to-run basis in terms of AUP (3.10e $5+/-4.2 \%)$ and retention time (RT) (7.59 +/- 0.03 mins) (Figure 3.4). As a comparison, SOP's at industrial laboratories consider $\pm 40 \%$ of the average internal standard ion signal as an acceptable range for a sample analysis [65]. Most importantly, the internal standard was a strong indicator of the instrument's performance on a run-to-run basis. Drug preparations of VSe7 were spiked with the pre-determined amount of caffeine and analyzed at 30 min intervals for $6.5 \mathrm{hrs}$ (Figure 3.5). Between 60 and 150 mins it was observed that salt had accumulated on the ESI tip and was causing poor spray. The HPLC was not the source of error due to the consistency of the RT. The poor spray is is reflected by the AUP of caffeine and a diminished TIC. Had the internal standard not been added to a drug preparation and a sharp decrease in VSe AUP was observed, one may have falsely assumed that those particular samples may have contained lower concentrations of VSe.

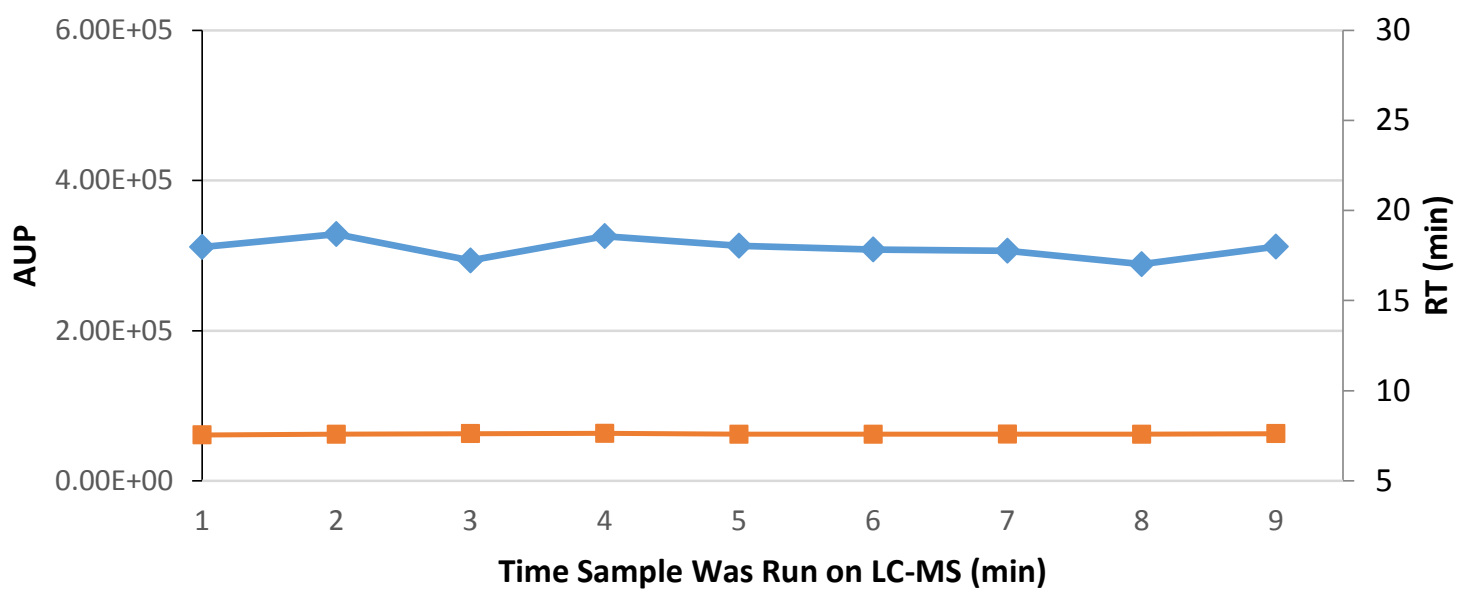

Figure 3.4: Caffeine AUP (blue) and RT (orange) reproducibility as an internal standard. 


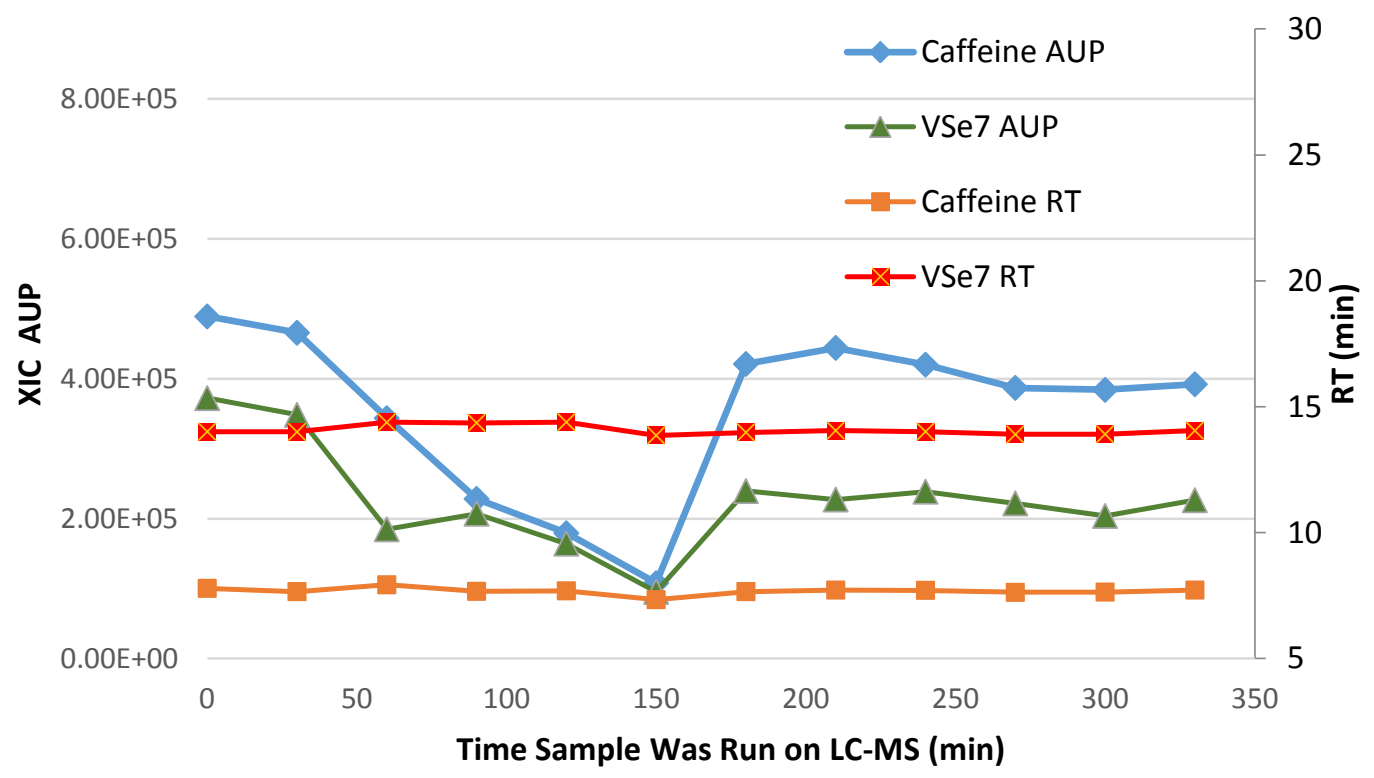

Figure 3.5: Caffeine as an indicator of LC-MS performance.

\subsection{Long-term stability of VSe1 and VSe7}

VSe1 and VSe7 are two of the lead VSe candidates for OVt and vaccine production (respectively). Each compound was dissolved in dimethylsulfoxide (DMSO) (Sigma) and diluted to various concentrations $(500 \mu \mathrm{M}-500 \mathrm{nM})$. To confirm the drug presence and identity, $50 \mu \mathrm{M}$ solutions were subjected to an accurate mass scan by direct infusion on the QStar XL instrument. The observed $[\mathrm{M}+\mathrm{H}]^{+1}$ peak at $229.0038 \mathrm{Th}$ is comparable to the expected $\mathrm{m} / \mathrm{z}$ of 228.9823 Th for VSel (Figure 3.6). The observed $[\mathrm{M}+\mathrm{H}]^{+1}$ peak at $171.0127 \mathrm{Th}$ is approximate to the expected $\mathrm{m} / \mathrm{z}$ of $171.0051 \mathrm{Th}$ for VSe7. VSe1 is a more unstable molecule as indicated by the sequential loss of $\mathrm{HCl}$ and $\mathrm{CO}$ fragments to produce the respective chlorinated fragments at $193 \mathrm{Th}$ at $165 \mathrm{Th}$ without CID. Both VSe1 and VSe7 samples often produced respective $[\mathrm{M}+\mathrm{Na}]^{+1}$ ions at 251 Th and 193 Th. 


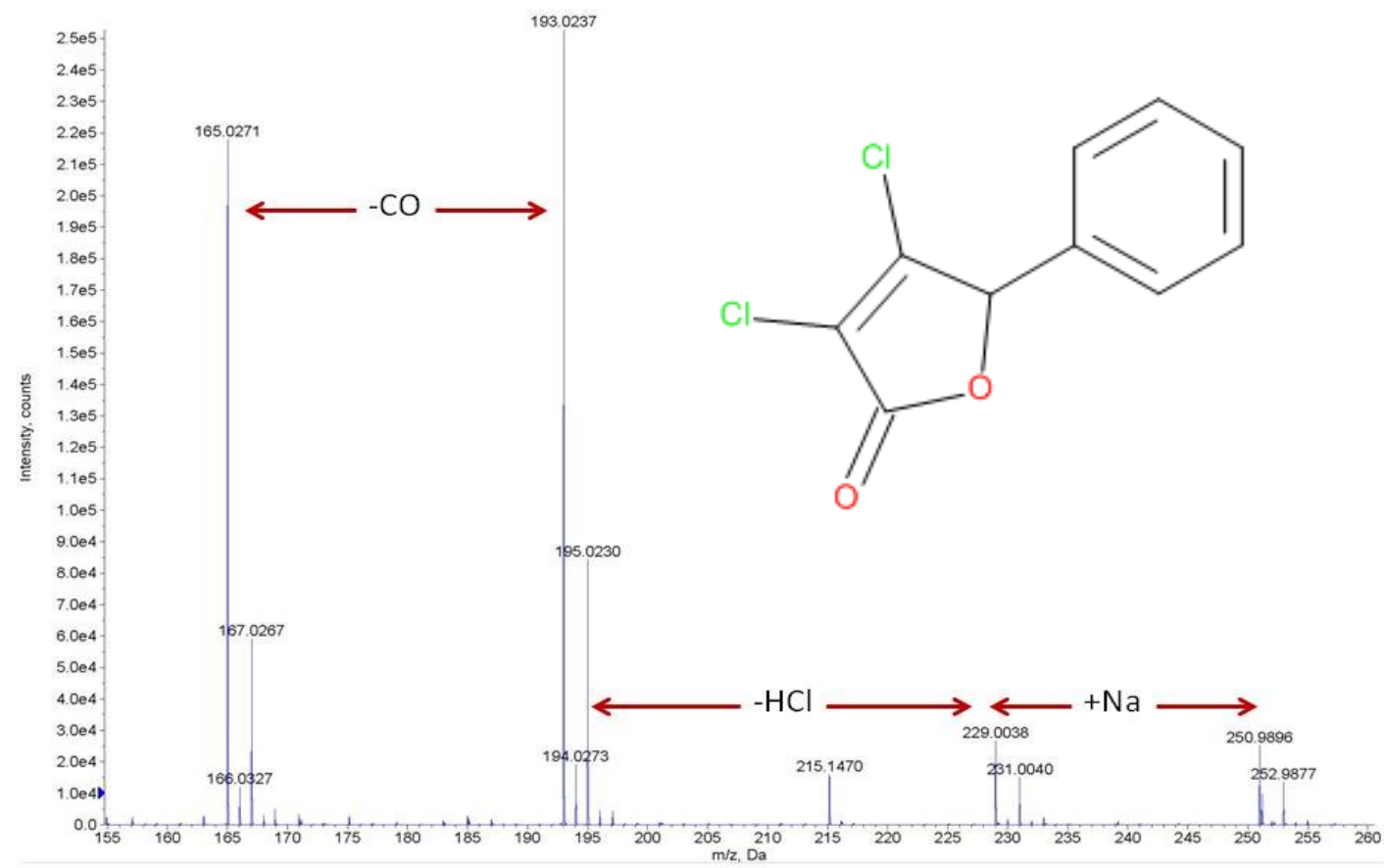

Figure 3.6: Direct infusion accurate mass spectrum of $50 \mu \mathrm{M}$ VSe1.

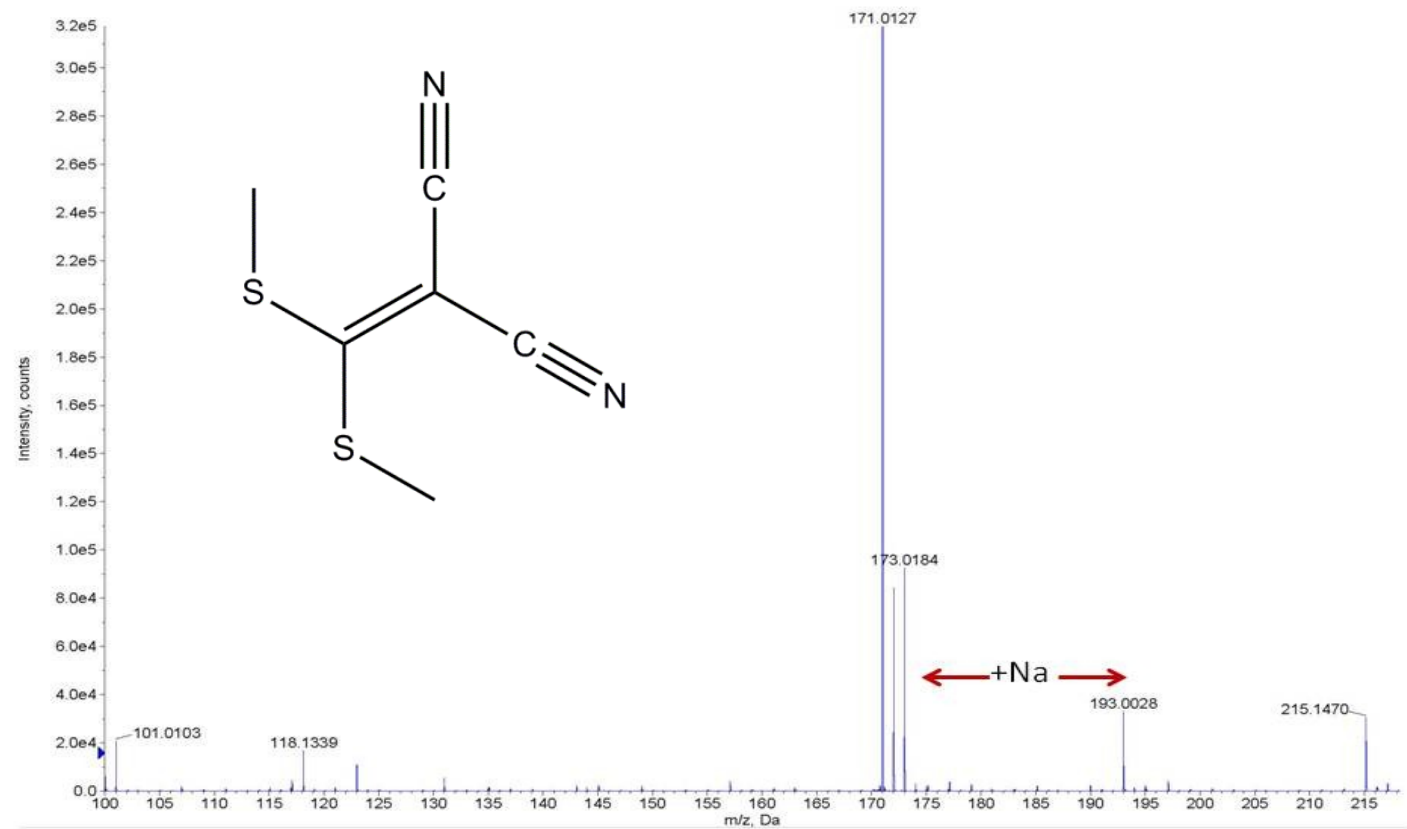

Figure 3.7: Direct infusion accurate mass spectrum of $50 \mu \mathrm{M}$ VSe7. 
The majority of the VSe analogue structures are chlorinated and often underwent the neutral loss of $\mathrm{HCl}(36$ and $38 \mathrm{Da}$ ) to produce singly chlorinated fragments. It was useful knowledge to understand the mechanism through which these compounds lost $\mathrm{HCl}$. A mechanism was proposed by Professor Jeff Manthorpe that caused the loss of the $\beta-\mathrm{Cl}$ as opposed to the $\alpha-\mathrm{Cl}$ (Figure 3.8). Resonance stabilization of the carbocation leads to Fragmentation Channel 1 which produces a 91 Th fragment. Fragment channel 2 is caused by the loss of CO that further splits into three channels (A, B, C). Further loss of $\mathrm{HCl}$ produces a $129 \mathrm{Th}$ fragment via Channel A. Channel B begins with the carbonyl lone pair inductively pushing electrons to stabilize the carbocation and yields fragments of $105 \mathrm{Th}$ and $77 \mathrm{Th}$ (a benzene ring). Channel 2C begins with resonance stabilization of a four-membered ring the consecutive losses of $\mathrm{CO}$ and a $\mathrm{Cl}^{-}$radical to produce fragments of $137 \mathrm{Th}$ and $102 \mathrm{Th}$. A product ion scan of $193 \mathrm{Th}$ using a CE of $25 \mathrm{eV}$ showed the rapid loss of $28 \mathrm{Da}(\mathrm{CO})$ to provide more support for the proposed mechanism (Figure 3.9) and detected several of the predicted cationic fragments. Many VSes produced a $91 \mathrm{Th}$ fragment that is commonly identified as the tropylium ion, but this was not the case for VSe1. The tropylium ion was observed for $70 \%$ of the VSe analogues and was used as a signature for VSe-specific metabolite identification (Chapter 4). 


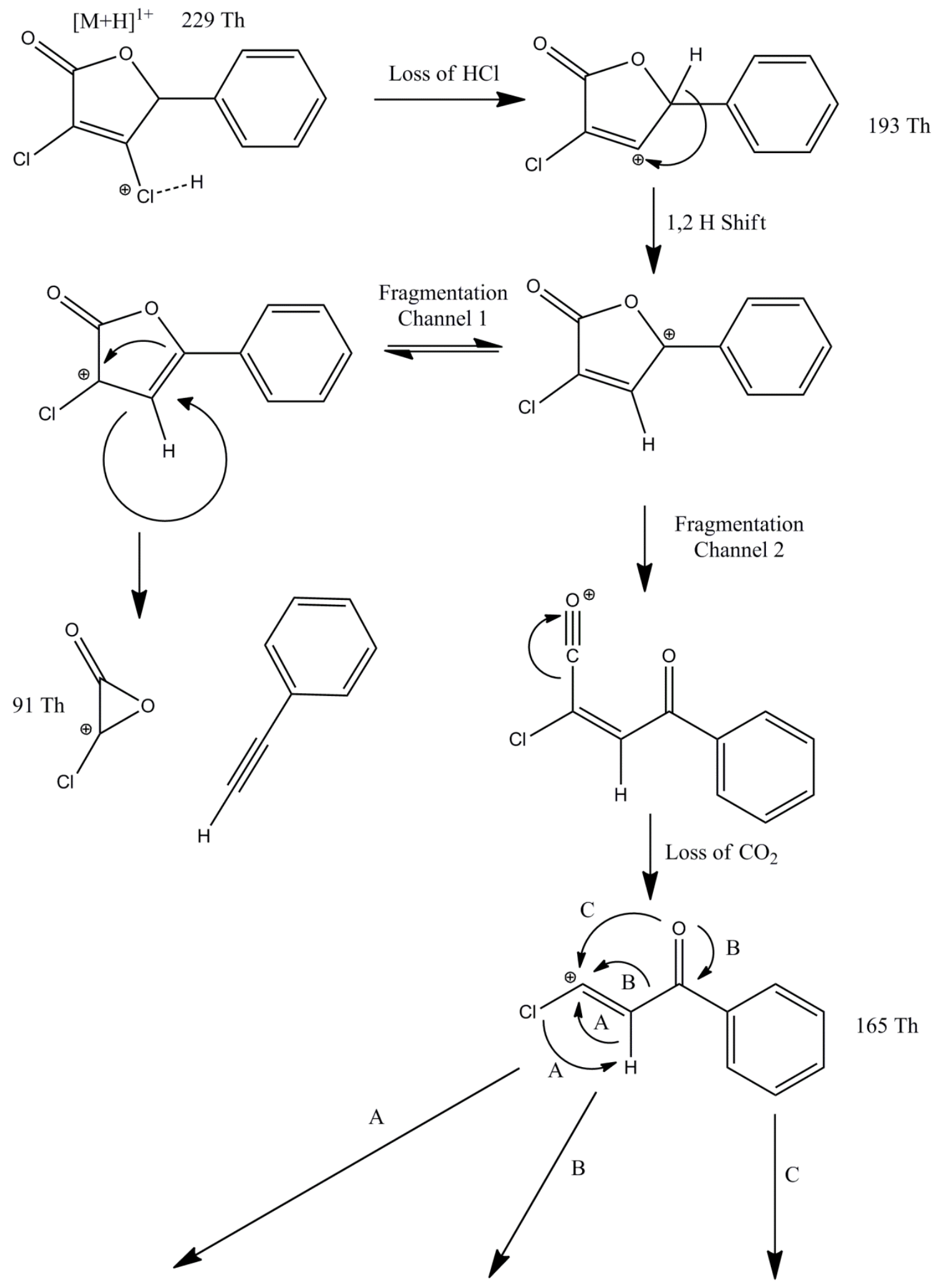




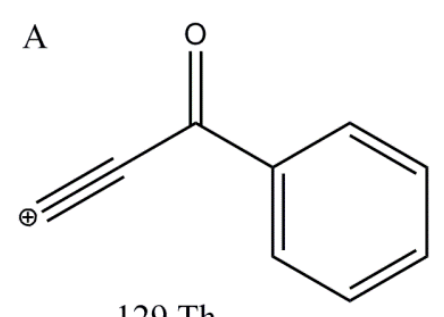

$129 \mathrm{Th}$
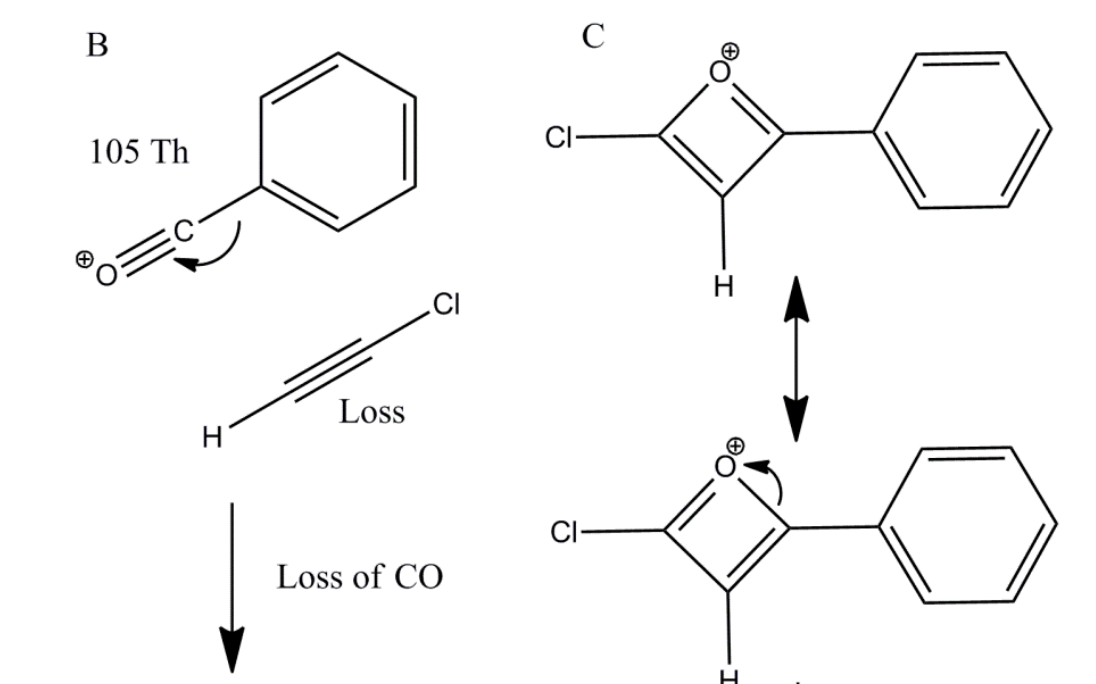<smiles>c1ccccc1</smiles>

$77 \mathrm{Th}$
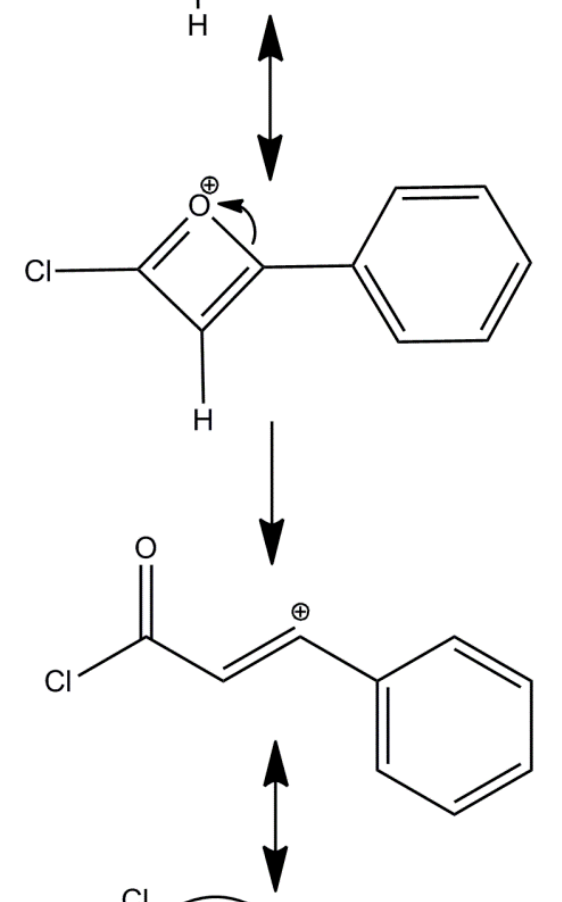

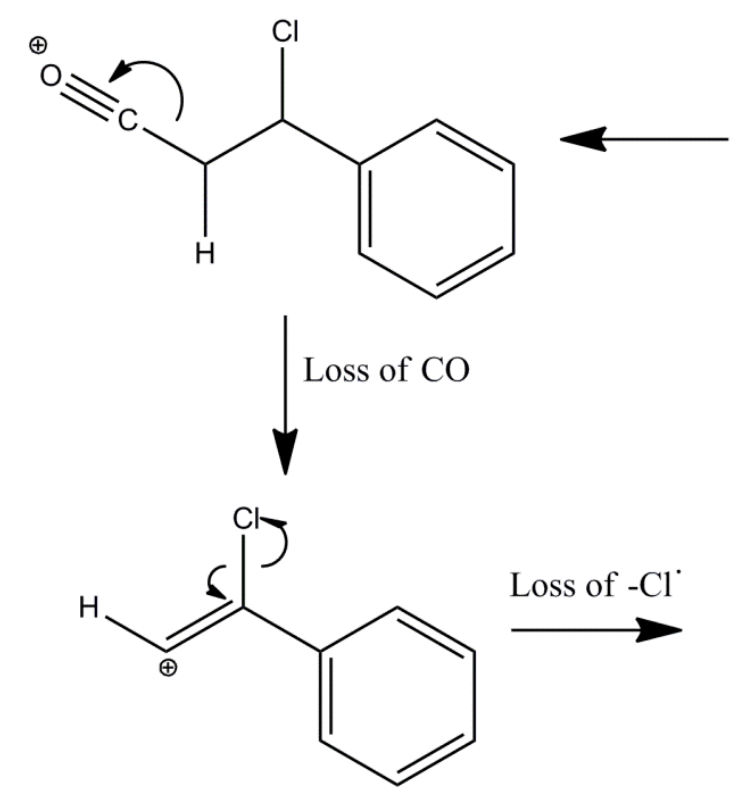

$137 \mathrm{Th}$

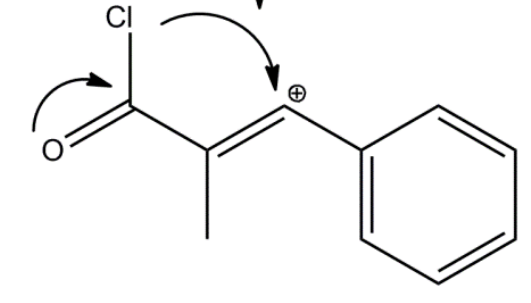

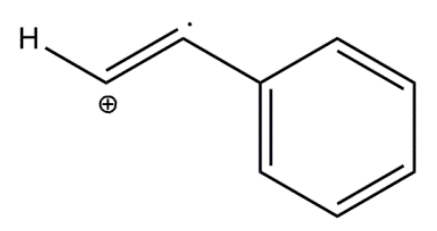

$102 \mathrm{Th}$

Figure 3.8: The proposed mechanism through which VSes lose the HCl neutral fragment. 


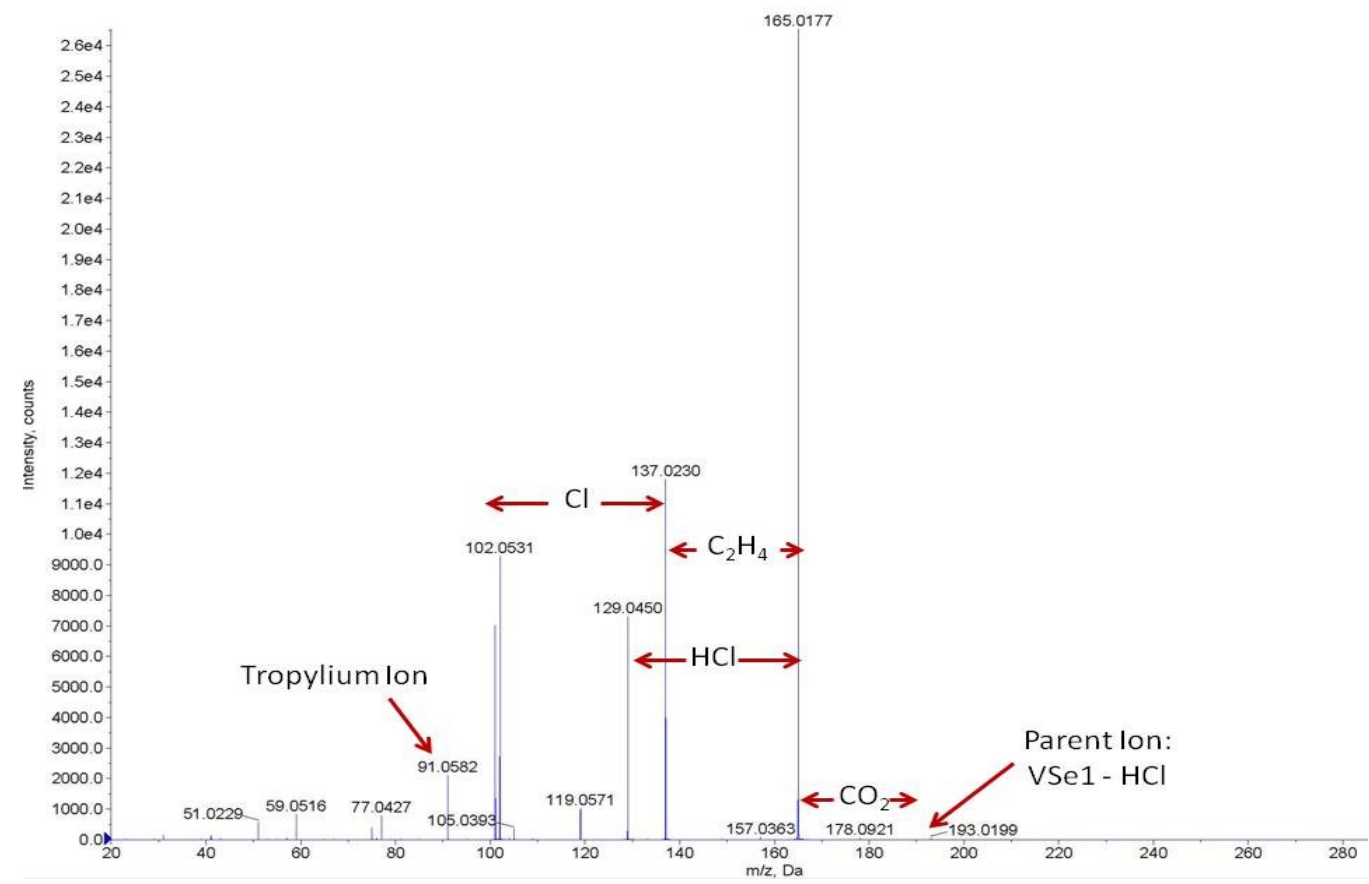

Figure 3.9: Product ion scan of the 193 Th VSe1 Fragment ( $50 \mu M)$.

An optimal VSe concentration was determined to monitor the small molecule stability using LC-MS on the QStar XL mass spectrometer. As a poor example, a VSe1 concentration of $500 \mu \mathrm{M}$ caused over-saturation of the detector, tailing of the XIC and substantial carryover in the following blank run (Figure 2.10). Detector saturation is indicated by the analyte's ${ }^{36} \mathrm{Cl}$ to the ${ }^{38} \mathrm{Cl}$ forms heavy isotope ratio being significantly increased from the expected chlorine isotope ratio of $33 \%$ between the ${ }^{36} \mathrm{Cl}$ to the ${ }^{38} \mathrm{Cl}$ forms of the parent ions in Figure 3.6. It was determined that a dilution to $12 \mu \mathrm{M}$ was suitable for VSe1 and $4.8 \mu \mathrm{M}$ for VSe7 due to its improved stability in solution (Figures 3.11 and 3.12). These concentrations provided chromatograms with high resolution and unsaturated mass spectra. 

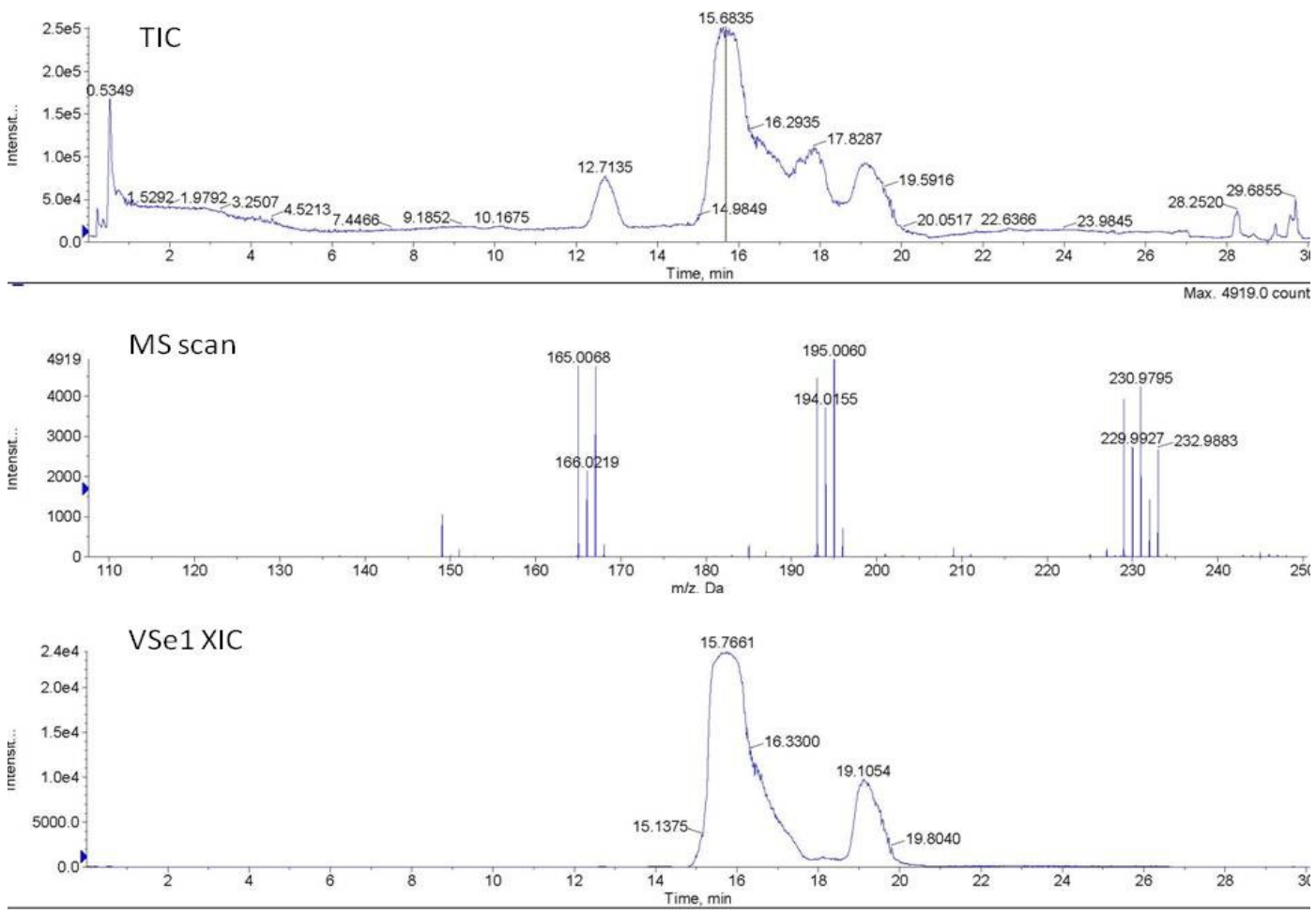

Figure 3.10: LC-MS of a $500 \mu \mathrm{M}$ VSe1 solution beyond the dynamic range of the detector.
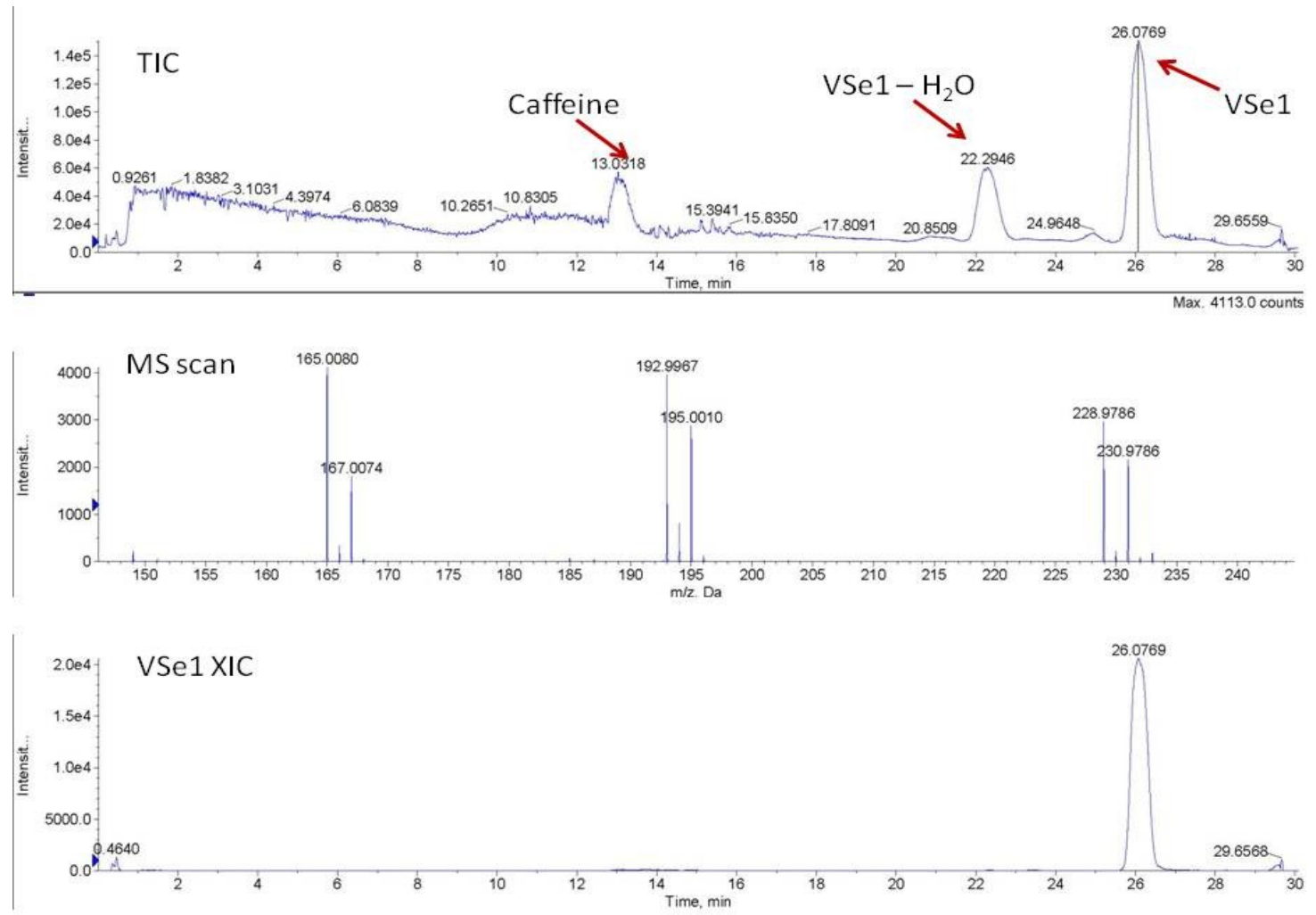

Figure 3.11: LC-MS analysis of a $12 \mu M$ VSe1 solution that is suitable for QC experiments. 

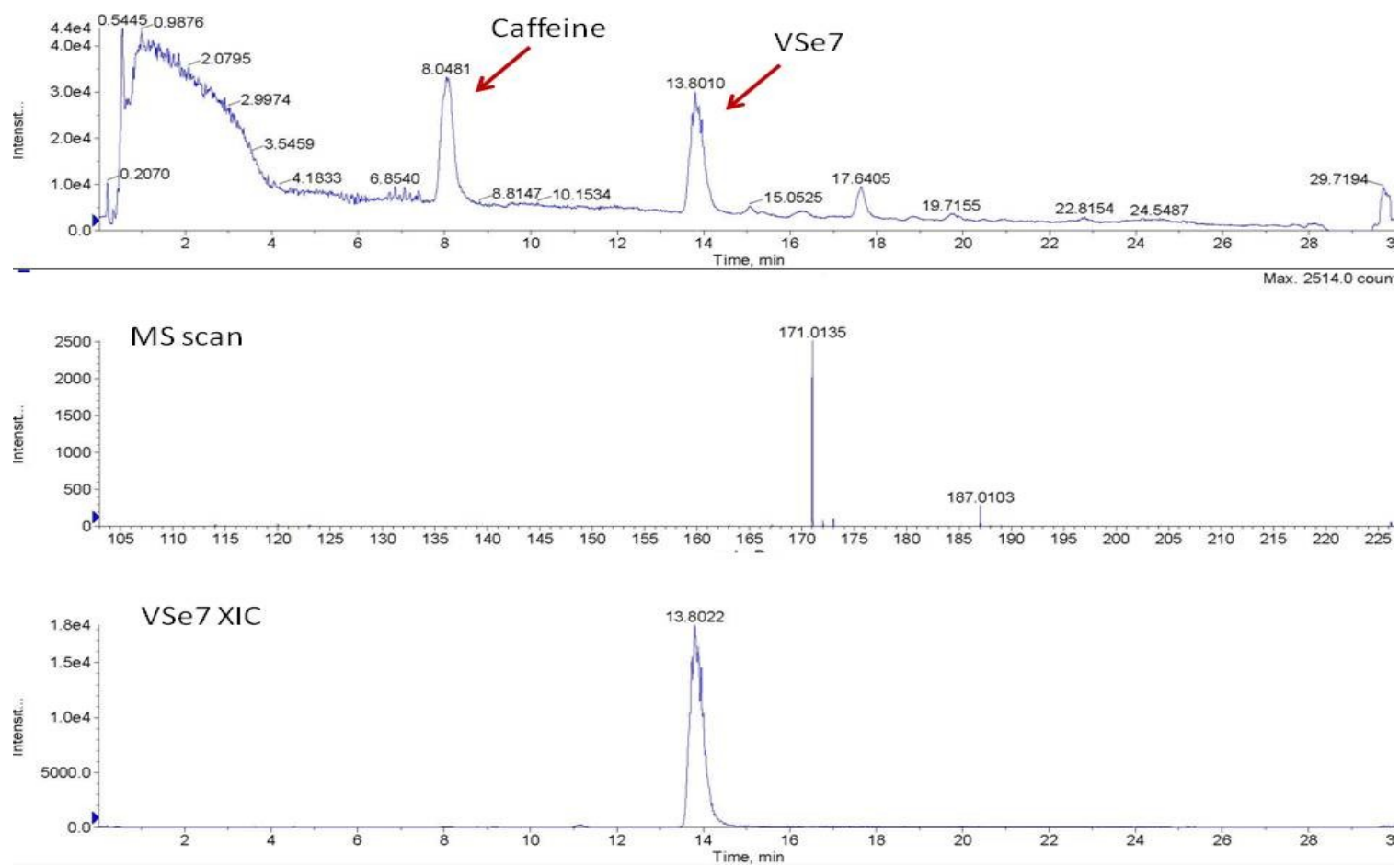

Figure 3.12: LC-MS analysis of a 4.8 $\mu \mathrm{M}$ VSe7 solution that is suitable for QC experiments.

The first application of this simple LC-MS method was to monitor the stability of VSe1 and VSe7 under long-term storage conditions. Aliquots of these drugs were commonly stored frozen in DMSO. Ensuring that the molecules were stable under these conditions for a long period of time would be beneficial knowledge for future experiments. VSe1 and VSe7 powders were solubilized in DMSO to a concentration of $24 \mathrm{mM}$ and $70 \mu \mathrm{L}$ aliquots were transferred to separate tubes. Ten microlitres of the aliquot was diluted in $990 \mu \mathrm{L}$ of $2 \%(\mathrm{ACN})$ in $0.1 \% \mathrm{FAw}$ and allowed to resolubilize in the aqueous solvent for 10 mins. This was followed by another dilution into the same diluents. VSe1 was diluted 1:20 and VSe7 was diluted 1:50 to produce concentrations of $12 \mathrm{uM}$ and $4.8 \mu \mathrm{M}$ respectively. From these solutions, $19 \mu \mathrm{L}$ was transferred in triplicate to $2 \mathrm{~mL}$ amber screw thread HPLC vials (Fisher) with $0.5 \mathrm{~mL}$ spring conical inserts 
(Canadian LifeScience). One microlitre of caffeine spike was added to each technical replicate and relatively quantified by the same LC-MS full mass scan as previously described. The DMSO stocks were frozen at $-80^{\circ} \mathrm{C}$ and one aliquot of each drug was analyzed immediately after one freeze-thaw cycle to compare the drugs' stability. This is important because drug preparations in pre-clinical research are often used repeatedly between freeze-thaw cycles and it is assumed this process does not affect stability. The average AUP ratio of VSe to caffeine was calculated using MultiQuant software. Relative quantitation analysis was performed at various time-points at up to 8 months of frozen storage at $-80^{\circ} \mathrm{C}$ (Figures 3.13 and 3.14). This experiment encouragingly showed that VSes are stable in frozen DMSO over a period of multiple months with VSe1 observing only a $23 \%$ decrease in quantity. It remains to be seen if this stability is maintained over longer periods of time. This experiment also demonstrated that VSe1 is stable between one freeze-thaw cycle as indicated by the close proximity of the two points at $\mathrm{t}=0$. VSe7 does see a $39 \%$ decrease in quantity after one cycle.

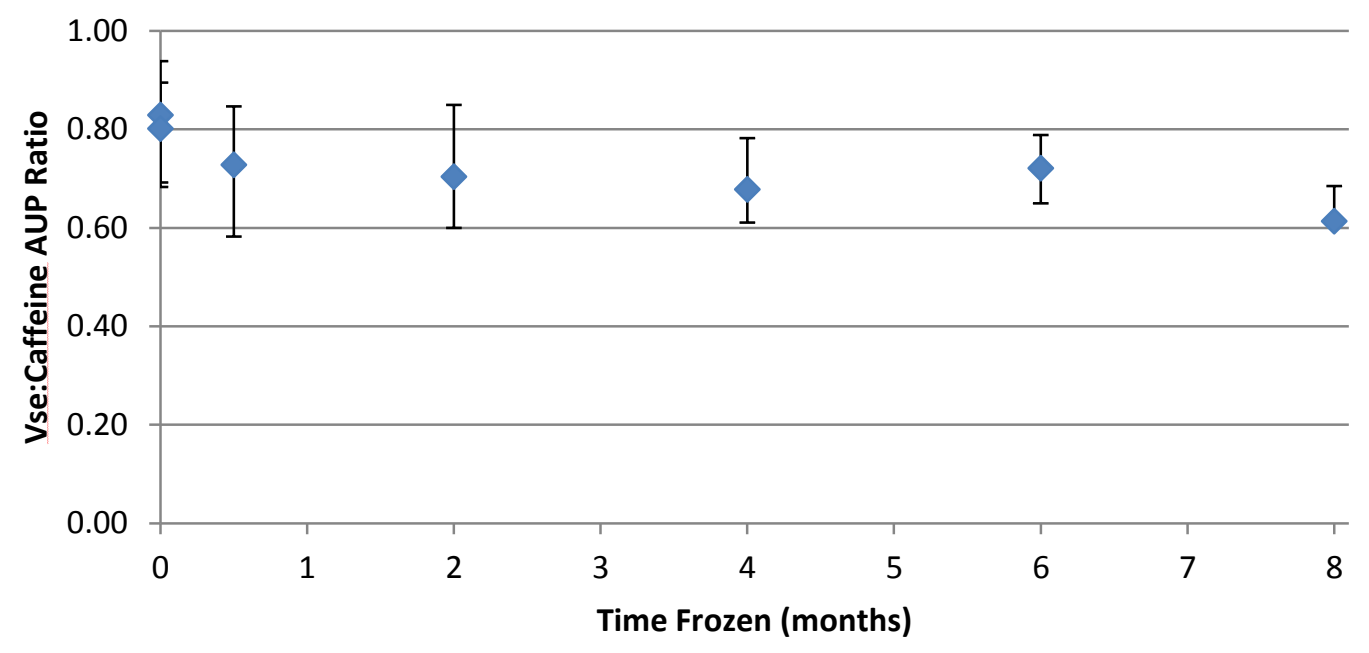

Figure 3.13: VSe1 frozen long-term stability. 


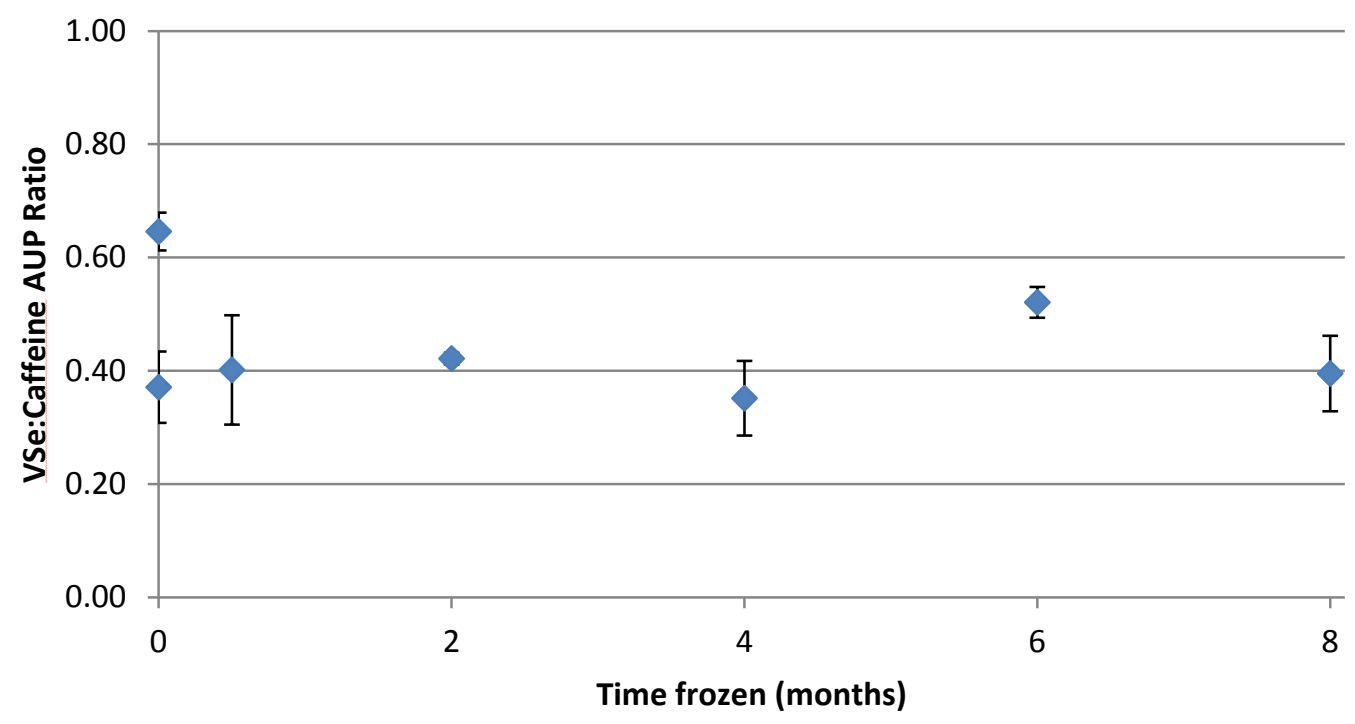

Figure 3.14: VSe7 frozen long-term stability.

\subsection{VSe7 stability under experimental conditions}

VSe7 is most often used to enhance influenza viral replication within fertilized chicken eggs for the purposes of vaccine production. Research showed inconsistent results since the peak dose (the dose of VSe7 that provides optimal viral titer) changed between experiments. With aspirations of transferring the VSe technology to industrial collaborators, it became a priority to determine if the drug preparation was the source of discrepancy. An MRM LC-MS method (refer to Chapter 4 for method development) was developed for VSe7 including a 15 min HPLC Gradient A for increased assay throughput. Relative quantitation was carried out on the 4000 Qtrap instrument. A 12 $\mathrm{mM}$ DMSO solution of VSe7 was prepared and diluted to $24 \mu \mathrm{M}$ in $37^{\circ} \mathrm{C} \mathrm{PBS}(\mathrm{pH}=7.4)$. These conditions were selected to mimic the incubation of VSe7 in chicken egg amniotic fluid. The VSe7 solution was incubated on a shaker at $37^{\circ} \mathrm{C}$ and $20 \mu \mathrm{L}$ aliquots were quantified every 20 mins for 160 mins. The samples were subjected to a freeze-thaw 
cycle every 160 mins to confirm the drug's stability to a total time of 8 hrs (Figure 3.15). The experiment was experimental triplicate. The experiment confirmed that VSe7 is stable in PBS at $37^{\circ} \mathrm{C}$ which can be extended to the chicken egg experiments. Additionally, the molecule is stable through freeze-thaw cycles which contradicts the speculation introduced in Figure 3.14.

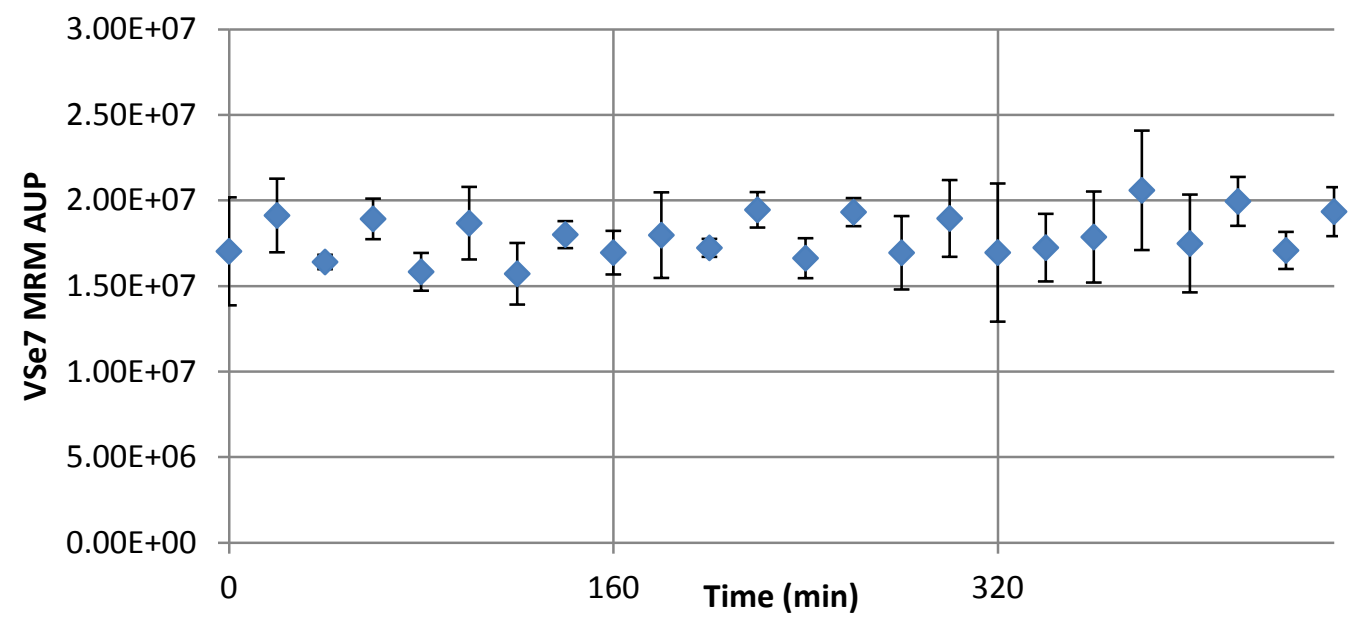

Figure 3.15: VSe7 stability under experimental egg conditions.

\subsection{Absolute quantitation of VSe7 drug preparation and method validation}

Ensuring the consistent and reproducible preparation of VSe7 will ease the transfer of VSe technology to other laboratories. The previously described QC experiments have made use of relative quantitation to monitor the stability of VSe7 over time. This is not as useful for when the concentration of VSe7 in a drug preparation needs to be determined prior to shipment to external collaborators. Absolute quantitation method development was necessary by creating a standard curve comparing the VSe7:caffeine AUP ratio to VSe7 concentration (Figure 3.16). This was performed in experimental triplicate using the 30 min sensitizer method on the QStar XL instrument. 
The high $\mathrm{R}^{2}$-value (0.9853) with a linear fit indicates that concentrations of VSe7 within this range are encompassed by the detector's linear range that is suitable for absolute quantitation. Three different drug preparations were diluted to $50 \mu \mathrm{M}$ in $2 \%$ ACN in FAw and subjected to absolute quantitation using the same method (Table 2.1).

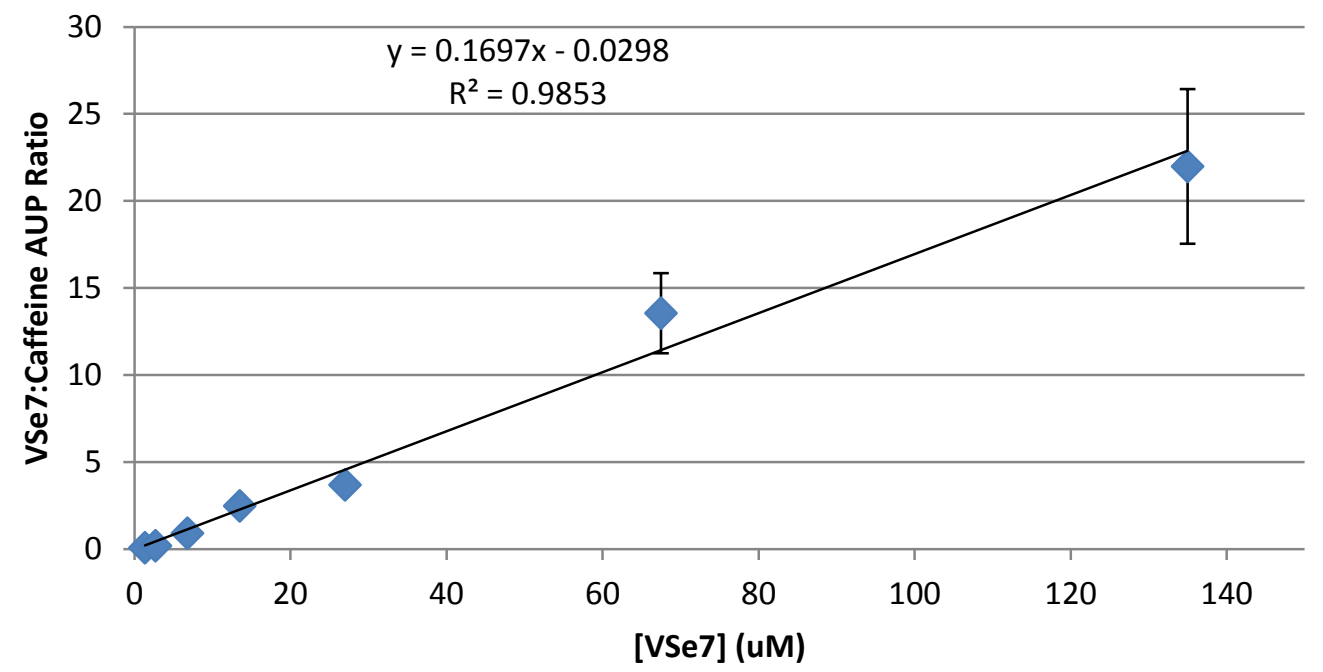

Figure 3.16: VSe7:Caffeine AUP Ratio standard curve.

Table 3.1: The absolute quantitation of various VSe7 preparations

\begin{tabular}{l|c|c}
$\begin{array}{l}\text { VSe7 Preparation } \\
\text { Name }\end{array}$ & $\begin{array}{c}\text { VSe7 Quantitation } \\
\text { Experiment 1 (mM)* }\end{array}$ & Experiment 2 (mM)** \\
\hline JW & $64 \pm 6$ & $59 \pm 4$ \\
JH & $51 \pm 4$ & $59 \pm 4$ \\
RA & $48 \pm 8$ & $51 \pm 4$ \\
*Drug preparation was diluted 1:1000 & \\
**Drug preparation was diluted 1:4000 & \\
***Drug preparations were expected to contain a [VSe7] of $100 \mathrm{mM}$
\end{tabular}


The experimental drug preparations contained approximately $50 \%$ less VSe7 than the anticipated $100 \mathrm{mM}$. This result corresponded with lower than expected viral titer results that used the same drug preparations. The analysis was repeated for the same preparations and demonstrated comparable concentrations indicating that the absolute quantitation method is dependable when diluting to different analyte concentrations within the linear range. Despite the viral titer results, the lower than anticipated concentrations called the LC-MS quantitation method into question and led to method validation experiments.

Firstly, ensuring that the caffeine internal standard was not causing the source of discrepancy was critical (Figure 3.17). A $1 \mu \mathrm{L}$ caffeine spike was mixed with $19 \mu \mathrm{L}$ of $\mathrm{ddH}_{2} \mathrm{O}$ (column 1) or FAw (column 3) in technical triplicate. This can also be compared to column 2 that represents the amount of caffeine quantified from one vial containing 3 $\mu \mathrm{L}$ of caffeine spike and $57 \mu \mathrm{L}$ of $\mathrm{ddH}_{2} \mathrm{O}$ as opposed to the same injections from three separate vials. A comparison between columns 1, 2 and 3 demonstrates that the AUP of caffeine does fluctuate but not sufficiently to cause a $50 \%$ decrease in VSe7 concentration. This comparison also confirms that the caffeine is stable in acidic diluent. Columns 4 and 5 show that acidic diluent had no effect on the stability $10 \mu \mathrm{M}$ VSe7 solutions. In column 6, an equimolar concentration of VSe7 was analyzed in the presence of caffeine. This was testing the hypothesis that $\mathrm{VSe} 7$ and caffeine conjugated in the presence of each other due to their reactive functional groups. The AUP of caffeine does not significantly change when analyzed in the presence of VSe7 (comparing columns 3 to column 6) and VSe7 AUP does not change in the presence of caffeine (comparing column 5 to column 6), thus disproving the conjugation hypothesis. 


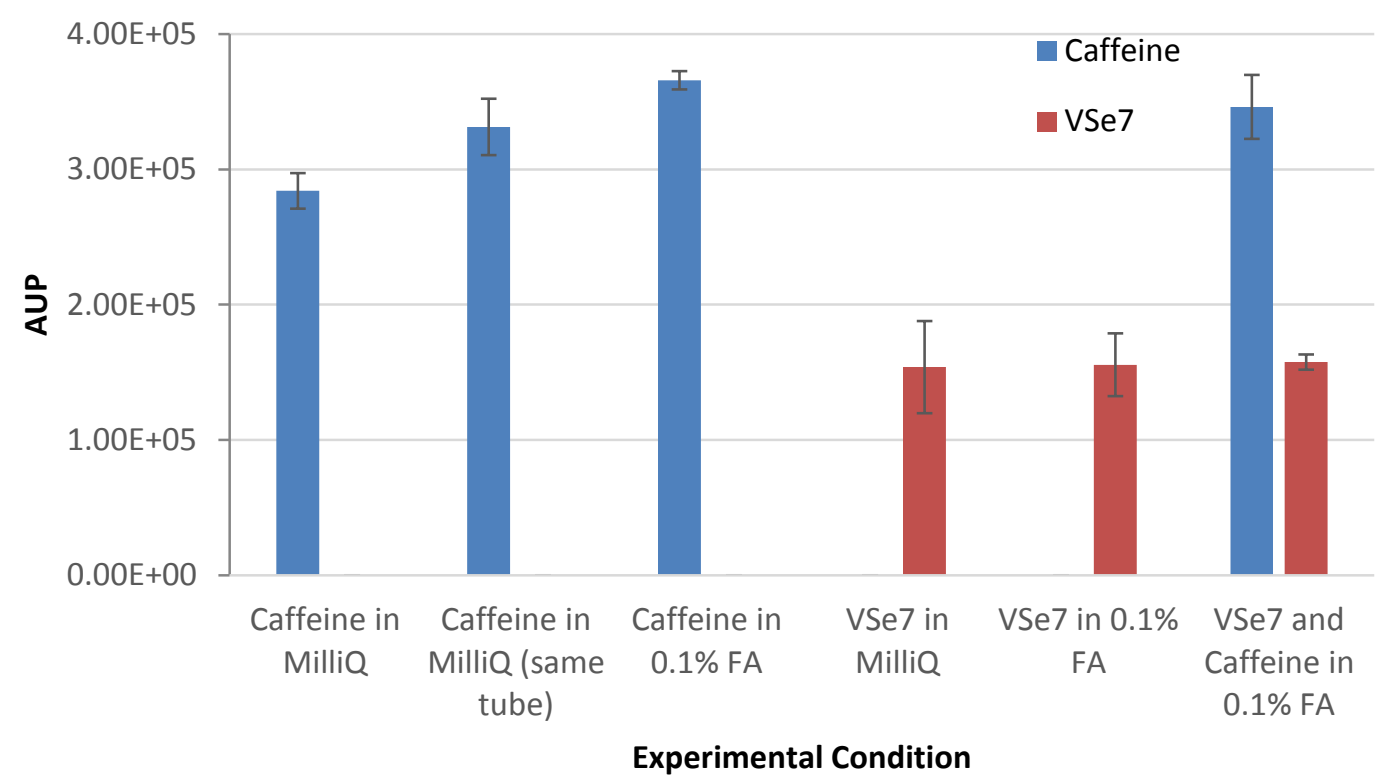

Figure 3.17: VSe7 absolute quantitation method validation of the internal standard and diluents.

The next component of the absolute quantitation method that required validation was the time allowed for VSe7 to resolubilize upon mixing the DMSO stock with $2 \%$ $\mathrm{ACN}$ in $0.1 \%$ FAw. It was speculated that not all of VSe7 was soluble before the next dilution into aqueous solvent had occurred because the solution visually became temporarily translucent. To test this hypothesis, VSe7 DMSO stock solution was diluted in experimental triplicate to $1 \mathrm{mM}$ in $2 \% \mathrm{ACN}$ in $\mathrm{FAw}$ and allowed to resolubilize for varying amounts of time at room temperature before being diluted to $10 \mu \mathrm{M}$. Different resolubilization times did not cause a significant difference in the amount of VSe7 detected, with the most being detected following a 10 min resolubilization time (Figure 3.18). An intriguing point is made when comparing column 1 to column 3 . The resolubilization time validation tests used the same VSe7 DMSO stock that had been used for the validation experiments in Figure 3.17 but the solution had been left at room 
temperature overnight. A $24 \mathrm{hr}$ incubation period at room temperature in DMSO caused a 63\% decrease in VSe7 AUP. This brought the drug's stability in DMSO into question.

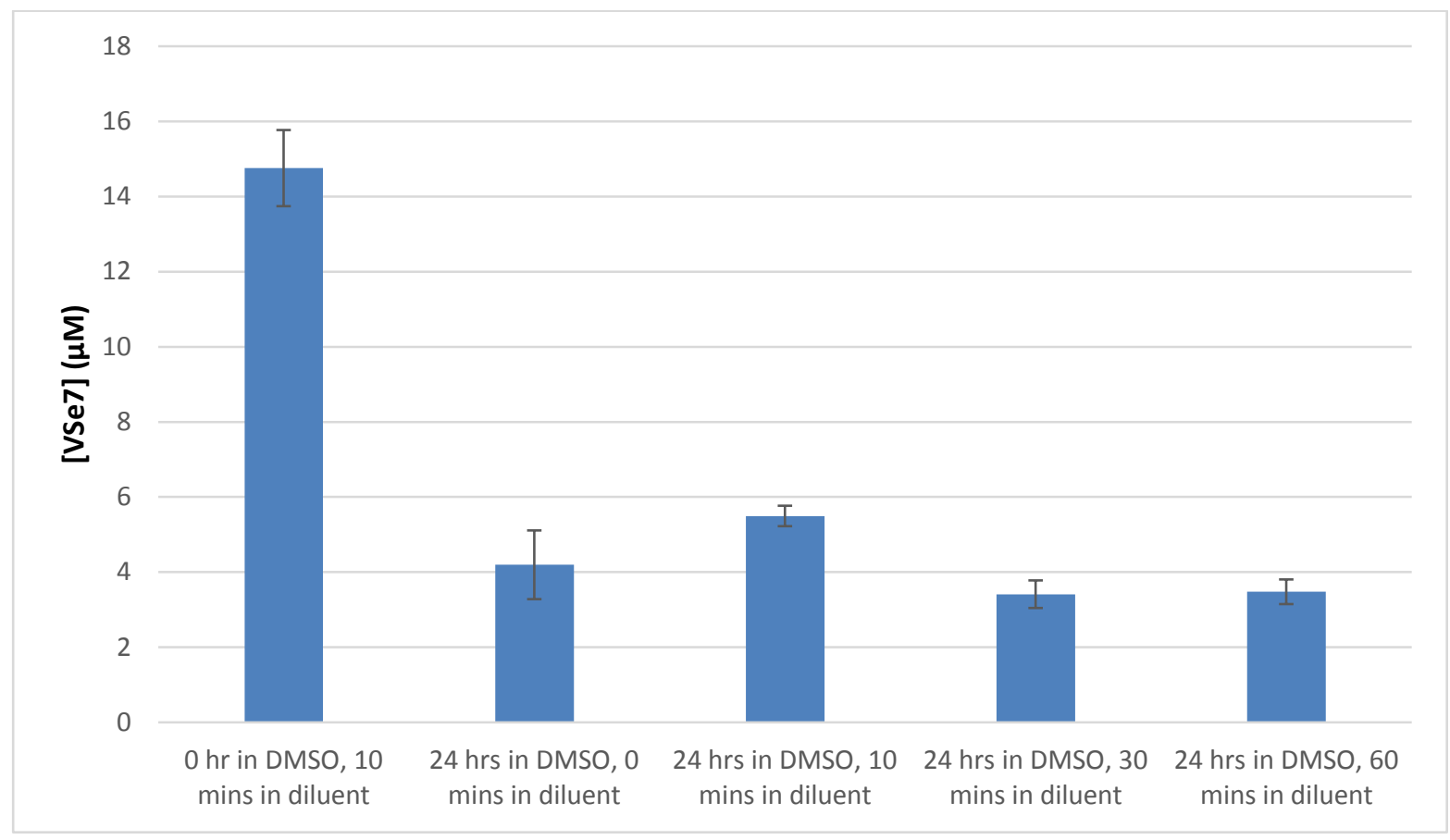

Figure 3.18: Validation of VSe7 resolubilization time in aqueous diluent.

A $100 \mathrm{mM} \mathrm{VSe} 7$ solution was prepared in DMSO and diluted in experimental triplicate to $1 \mathrm{mM}$ using DMSO as the diluent. At various time points of incubation at room temperature an aliquot was diluted to $10 \mu \mathrm{M}$ with $2 \% \mathrm{ACN}$ in FAw and absolutely quantified. Figure 3.19 demonstrates that VSe7 concentrations remain stable for the first few hours but after $24 \mathrm{hrs}$ in solution the VSe7 concentration had decreased by 55\%. This could be explained by an equilibrium between the drug's solubility and degradation occurring for the first few hours, but the drug had become entirely solubilized later in time so the degradation effect had shifted the equilibrium. Scanning the mass spectra for potential degradation products gave no insight into how VSe7 is breaking down and the TIC of the $24 \mathrm{hr}$ time point analysis shows no significant peaks arise in comparison to the 
$0 \mathrm{hr}$ analysis. The lower limit of the mass spectrum window was $103 \mathrm{Th}$ thus it could be speculated that the degradation products are smaller than this threshold and went undetected. Future studies should extend the furthest time point for further monitoring of VSe7 degradation and monitor a lower mass range. Further confidence is instilled in the absolute quantitation method because the VSe7 concentration at the early time-points is comparable to the expected concentration of $10 \mu \mathrm{M}$.

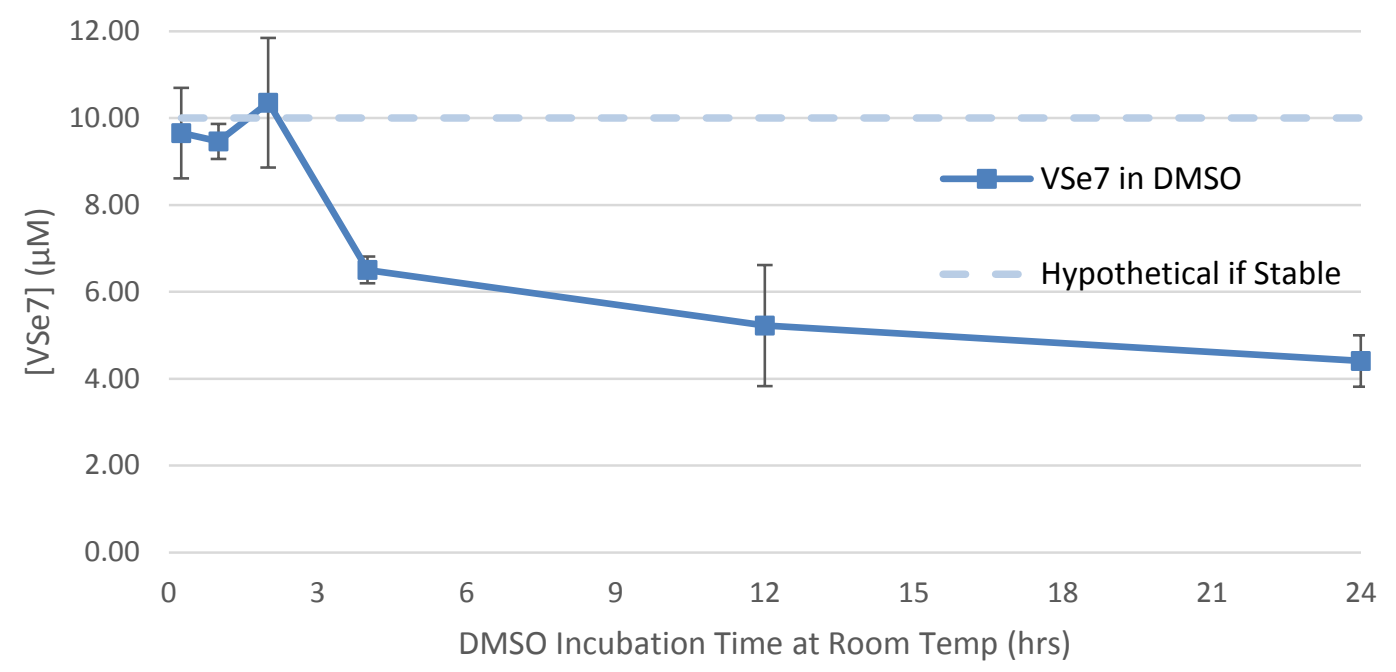

Figure 3.19: VSe7 stability in DMSO solution at room temperature.

\subsection{Conclusions}

This chapter demonstrated that LC-MS is a suitable method to perform regular QC experiments on VSes. Caffeine was selected as an appropriate internal standard and the spike volume and concentration were optimized to permit the relative quantitation of lead small molecules VSe1 and VSe7. Relative quantitation QC experiments revealed that both molecules are stable long-term frozen in DMSO and can undergo freeze-thaw cycles without a significant loss in concentration. Relative MRM quantitation showed that $\mathrm{VSe} 7$ is stable in $\mathrm{PBS}$ at $37^{\circ} \mathrm{C}$ which confirms that the influenza egg experimental 
conditions do not significantly affect the drug preparation. Finally, VSe7 was absolutely quantified in three different drug preparations using a linear standard curve. The drug preparations were quantified at approximately $50 \mathrm{mM}$; lower than the anticipated concentration of $100 \mathrm{mM}$. This led to method validation experiments that ultimately revealed the compound's poor stability in DMSO solution at room temperature; a contradiction to previous assumptions. These methods could be further validated by defining the LOB, LOD and LOQ for both VSe1 and VSe7. Applying UPLC technology to the front-end of the MS would significantly shorten the throughput of analysis by at least 5-fold. Another assumption that should be tested in future studies is the stability of the compounds in the powdered state. It is unknown if these compounds are stable upon exposure to light, humidity, oxidation or extreme temperatures which could cause discrepancies in down-stream research. In the future, VSes should be solubilized in a different organic solvent and frozen until use to minimize the time it remains in solution. 


\section{Chapter: In vitro and in vivo VSe stability}

\subsection{Structure-Activity Relationships}

Pre-clinical drug development is typically initiated using the hit-to-lead approach that begins with a high-throughput screen of diverse compound classes and the most active compound is called the hit. In most cases, the first hit is not the lead compound that ultimately enters clinical trials. This is because structure-activity relationship (SAR) analysis, or hit expansion, is a drug design tool that optimizes the structure of the initial hit in search of enhanced application-dependent properties among a library of hit analogues. Lead compounds with more favourable in vivo properties will improve the chances of clinical approval; thus the importance of SAR cannot be overlooked. The identification of new lead analogues is followed by lead optimization to identify structures that have favourable in vivo properties in terms of clearance and reduced offtargets [67]. In this chapter, LC-MS was used to assess the hit expansion process of a VSe library and initiate the lead optimization process to determine the in vivo fate of new lead compounds.

\subsection{MRM Method Development}

MRM scanning mode provides superior sensitivity relative to a simple full mass scan, but comes with a cost of time-consuming method development and optimization. The development process starts with accurate full mass scans of each VSe analogue. Obtaining accurate mass data confirms the identity of the VSe analogue and permits precise selection of the MRM parent ion, thus eliminating the co-fragmentation of 
isobaric compounds. Compound MD01145 was chosen as an example to demonstrate the MRM development process.

An accurate mass scan of MD01145 was collected within 5 ppm accuracy via direct infusion on the QStar XL instrument. The next step was selecting the MRM transition daughter fragments which required performing product ion scans. The collision energy was set to $15 \mathrm{eV}$ and the VSe daughter ions were detected and summed for approximately 100 scans ( 1 second scans). This was repeated for collision energies of 18 , $20,22,25,28,30,32,35$ and $40 \mathrm{eV}$. A comparison of the product ion spectra revealed the collision energy setting that produced the most intense daughter ions. In general, the lowest collision energy setting that caused the disappearance of the $[\mathrm{M}+\mathrm{H}]^{+1}$ peak, thus complete fragmentation of the parent ion, was most favorable because it limited further fragmentation of the primary daughter ions (Figure 4.1). The most intense daughter ion was termed the "quantitative transition" and was used for MRM chromatogram AUP. The second most intense daughter ion was called the "confirmatory transition". Alignment of the quantitative and confirmatory transition RT's was used to validate the detection of the quantitative transition signal in case it was a false-positive result arising from an isobaric non-VSe-specific compound. 


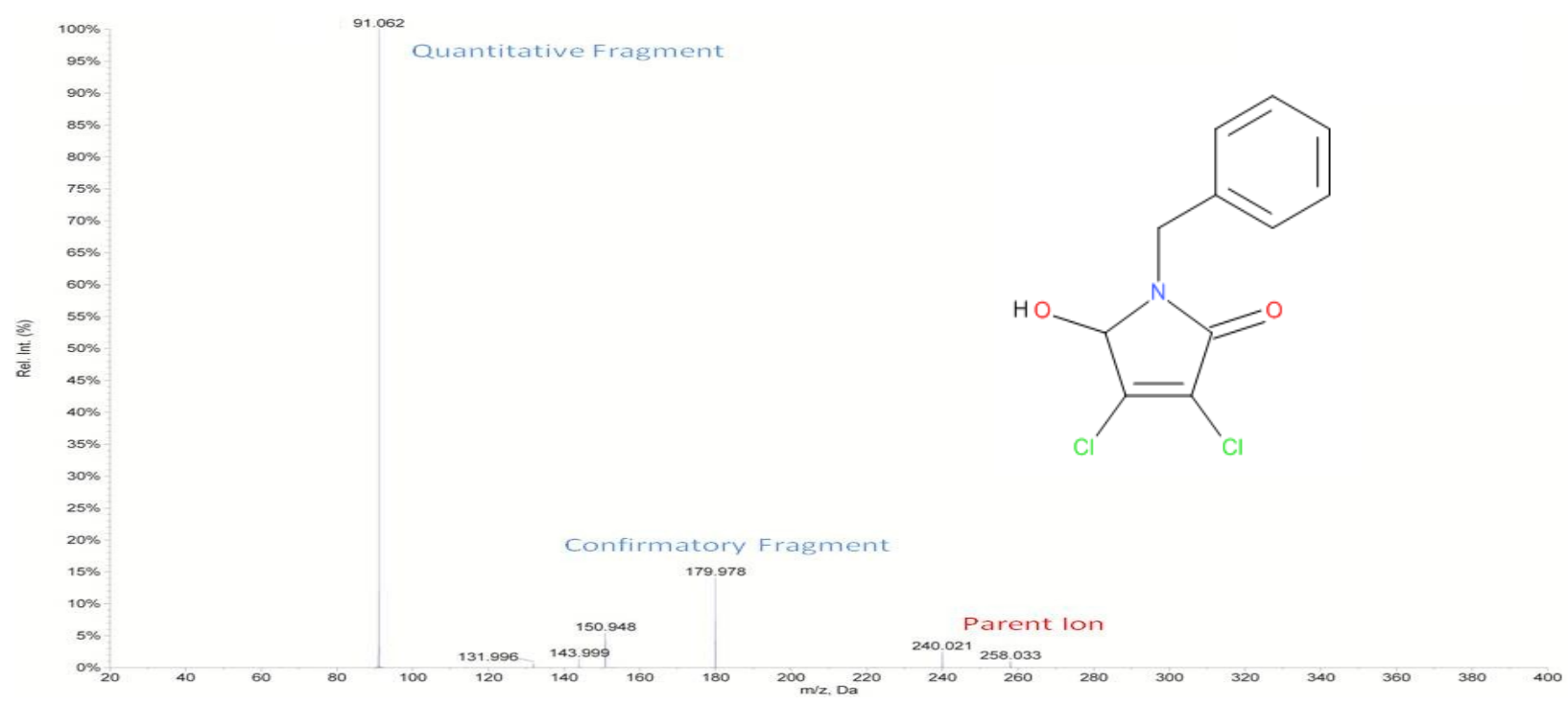

Figure 4.1: Product ion scan of MD01145 (compound 10) with a collision energy of $25 \mathrm{eV}$

The MRM transitions for each VSe were validated using LC-MS. The QStar XL mass spectrometer contains a TOF mass analyzer that is incapable of selecting daughter ions, and therefore is incapable of performing MRM. MRM experiments were performed on the 4000 Qtrap MS. $20 \mu \mathrm{M}$ VSe stock solutions in MeOH were diluted using 2\% ACN in $0.1 \%$ FAw and analyzed by LC-MRM. Some of the VSes needed further transition optimization on the QStar XL instrument because fragmentation behavior in the 4000 Qtrap instrument was not consistent with the QStar XL. MD01145 MRM quantitative and confirmatory transition chromatograms are shown in Figure 4.2. As expected, the quantitative transition showed more signal than the confirmatory transition at the same RT and the chromatography scheme produced a sharp elution profile. To gain a general sense of the VSe LOD using MRM, serial dilutions of MD01145 and MD03182 (Figure 4.3) were analyzed. Standard curves with a strong linear fit $\left(\mathrm{R}^{2}=0.99\right)$ and an LOD's as low as 19 fmol were achieved. 

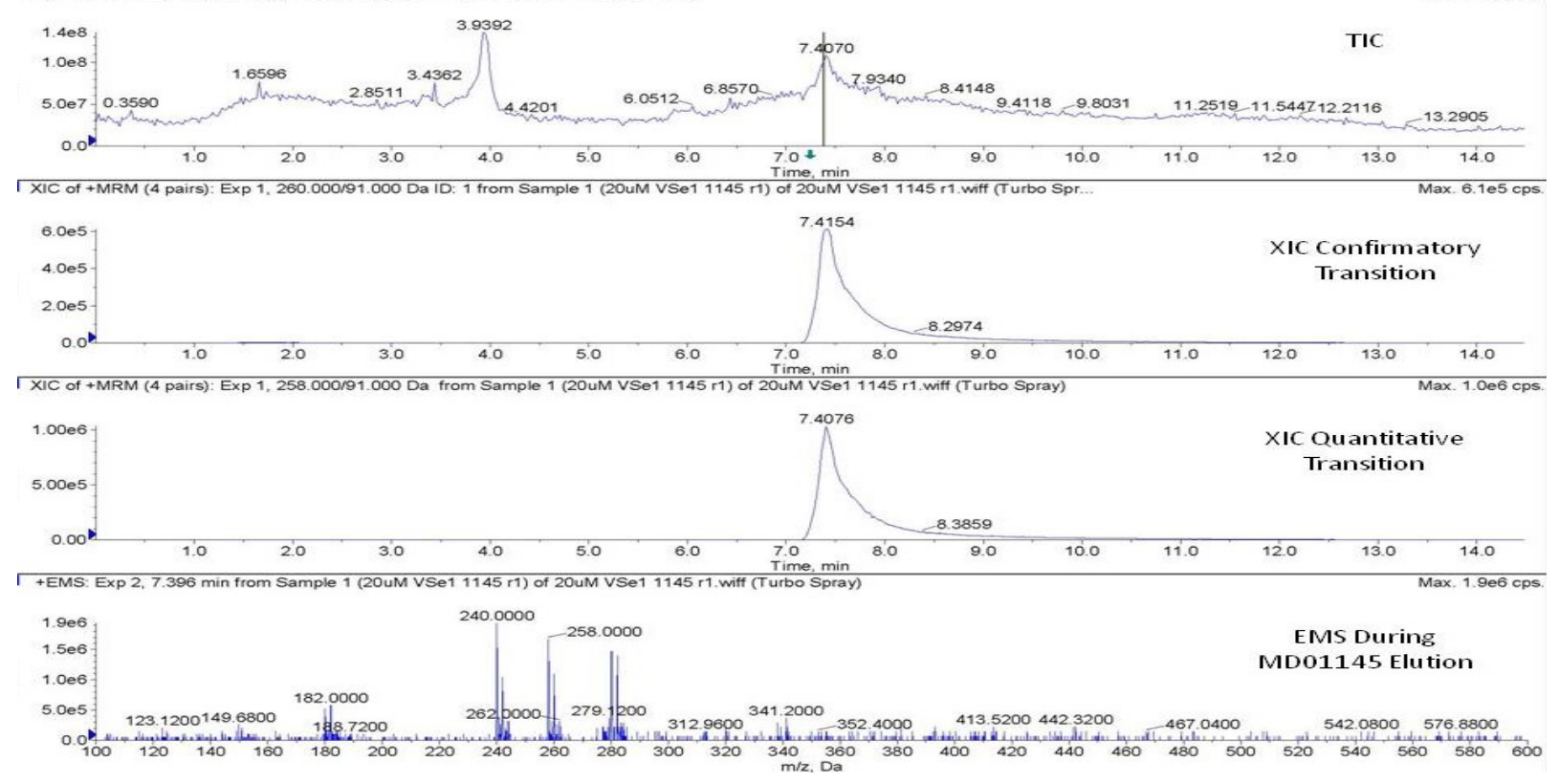

Figure 4.2: Validation of the MD01145 MRM transitions with LC-MS

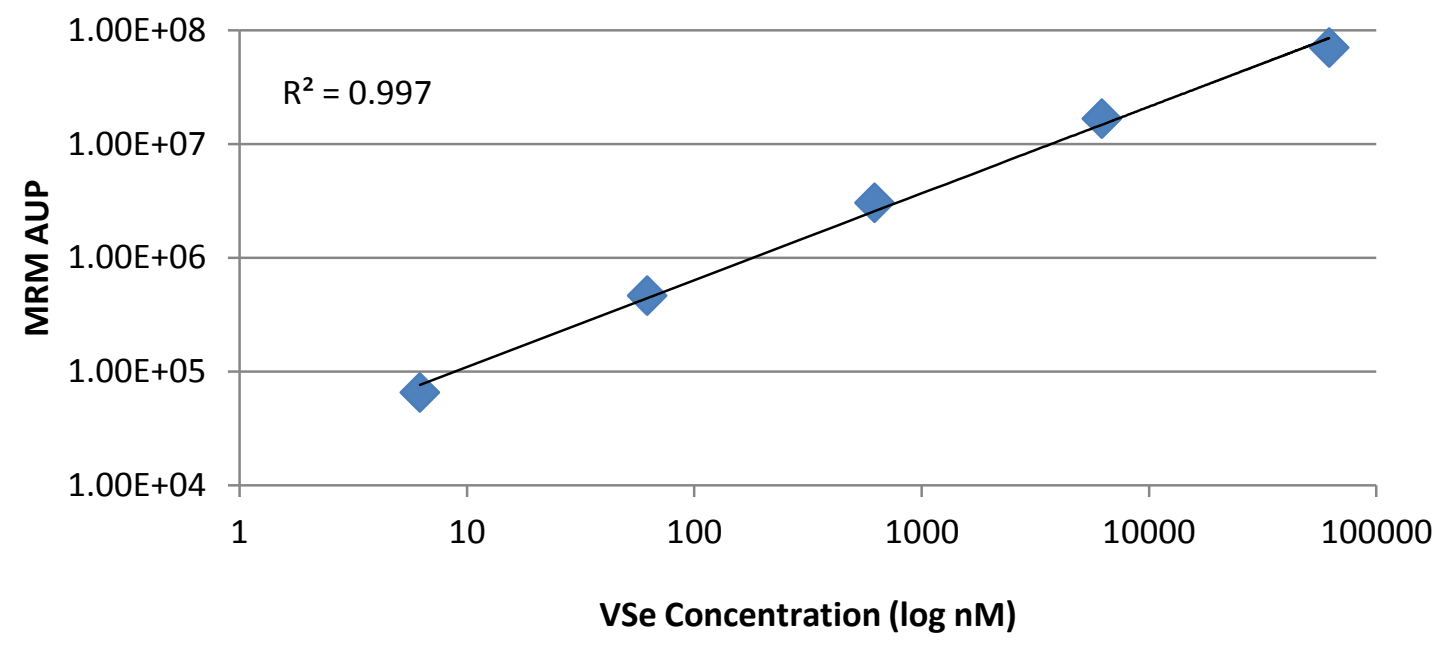

Figure 4.3: MD03182 Standard curve correlating concentration with MRM AUP

The MRM methods were intended for their application towards stability studies that would screen a library of more than $50 \mathrm{VSe}$ analogues. Analyzing the high quantity of samples associated with library screens placed a demand for high assay throughput. To meet this requirement, the HPLC gradient was shortened from 30 mins to 15 mins and the 
VSes were multiplexed into groups of three. Multiplexing criteria included eliminating the grouping of isobaric compounds or compounds with similar polarities so that VSe analogue co-elution and ion suppression were limited. HPLC gradient optimization was required because most VSes eluted between $50-80 \%$ organic mobile phase composition while some eluted at low organic mobile phase compositions; therefore prompting the development of Gradient B (see Chapter 2). As an example of how gradient optimization was necessary, quantitation of MD03182 was facilitated when Gradient A was substituted with Gradient B (Figure 4.4). The MRM transitions and gradient used to analyze each VSe analogue are presented in Table 4.1.

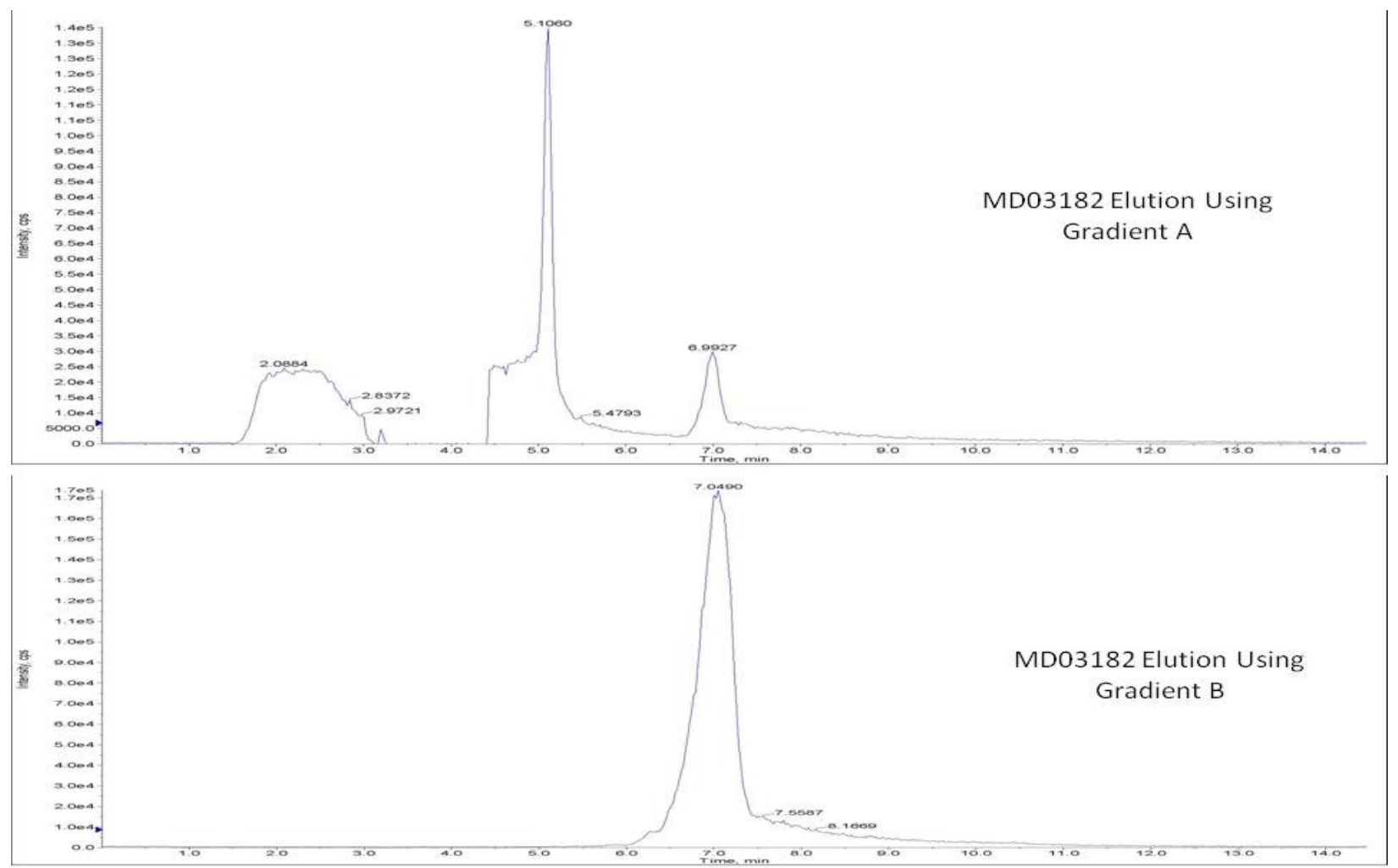

Figure 4.4: Improving the elution profile of MD03182 by switching to a sharper gradient B 
Table 4.1: VSe MRM parameters used to monitor drug stability

\begin{tabular}{|c|c|c|c|c|c|c|}
\hline \multirow[b]{2}{*}{ Compound } & \multicolumn{2}{|c|}{ Quantitative Transition } & \multicolumn{2}{|c|}{ Confirmatory Transition } & \multirow{2}{*}{$\begin{array}{c}\text { Collision } \\
\text { Energy (eV) }\end{array}$} & \multirow{2}{*}{$\begin{array}{c}\text { Chromatography } \\
\text { Gradient }\end{array}$} \\
\hline & $\begin{array}{c}\text { Parent } \\
\text { Ion }(\mathrm{m} / \mathrm{z})\end{array}$ & $\begin{array}{l}\text { Fragment } \\
\operatorname{lon}(m / z)\end{array}$ & $\begin{array}{c}\text { Parent } \\
\text { Ion }(\mathrm{m} / \mathrm{z})\end{array}$ & $\begin{array}{l}\text { Fragment } \\
\operatorname{lon}(\mathrm{m} / \mathrm{z})\end{array}$ & & \\
\hline 1 (VSe1) & 229.0 & 163.0 & 231.0 & 165.0 & 25 & A \\
\hline 2 & 318.9 & 209.0 & 318.9 & 237.0 & 18 & $\mathrm{~A}$ \\
\hline 3 & 300.1 & 193.0 & 302.1 & 195.0 & 20 & A \\
\hline 4 & 266.1 & 193.0 & 268.1 & 195.0 & 20 & A \\
\hline 5 & 152.9 & 61.0 & 152.9 & 117.0 & 22 & A \\
\hline 6 & 183.0 & 150.9 & 185.0 & 152.9 & 15 & $\mathrm{~B}$ \\
\hline 7 & 207.0 & 150.9 & 209.0 & 152.9 & 20 & $\mathrm{~B}$ \\
\hline 8 & 229.0 & 165.0 & 229.0 & 158.1 & 22 & A \\
\hline 9 & 168.0 & 132.0 & 170.0 & 134.0 & 20 & $\mathrm{~B}$ \\
\hline 10 & 258.0 & 91.1 & 260.0 & 91.1 & 20 & A \\
\hline 11 & 242.1 & 91.1 & 244.1 & 91.1 & 22 & A \\
\hline 12 & 272.0 & 91.1 & 274.0 & 91.1 & 20 & A \\
\hline 13 & 300.0 & 91.1 & 300.0 & 258.0 & 20 & A \\
\hline 14 & 256.0 & 91.1 & 256.0 & 178.0 & 17 & B \\
\hline 15 & 334.1 & 227.0 & 336.1 & 229.0 & 20 & A \\
\hline 16 & 190.1 & 91.1 & 190.1 & 112.0 & 18 & B \\
\hline 17 & 218.1 & 91.1 & 218.1 & 140.1 & 22 & B \\
\hline 20 & 240.1 & 91.1 & 240.1 & 133.0 & 27 & A \\
\hline 21 & 341.1 & 323.1 & 343.1 & 325.1 & 15 & B \\
\hline 22 & 182.0 & 146.0 & 184.0 & 148.0 & 25 & A \\
\hline 24 & 210.0 & 132.0 & 212.0 & 134.0 & 20 & B \\
\hline 25 & 208.0 & 150.9 & 210.0 & 152.9 & 23 & A \\
\hline 26 & 208.0 & 150.9 & 210.0 & 152.9 & 23 & A \\
\hline 27 & 206.0 & 134.0 & 208.0 & 134.0 & 23 & $\mathrm{~A}$ \\
\hline 28 & 281.1 & 178.0 & 283.0 & 180.0 & 25 & A \\
\hline 29 & 272.1 & 105.1 & 274.1 & 105.1 & 25 & A \\
\hline 30 & 286.1 & 164.0 & 286.1 & 117.1 & 20 & A \\
\hline 31 & 300.1 & 131.1 & 302.1 & 131.1 & 23 & A \\
\hline 33 & 282.0 & 133.1 & 284.0 & 133.1 & 20 & A \\
\hline 34 & 272.0 & 105.1 & 272.0 & 168.0 & 25 & A \\
\hline 35 & 334.0 & 167.1 & 336.0 & 167.1 & 25 & A \\
\hline 36 & 288.0 & 121.1 & 290.0 & 121.1 & 18 & A \\
\hline 37 & 274.0 & 107.1 & 276.0 & 107.1 & 30 & B \\
\hline 38 & 292.0 & 125.0 & 294.0 & 125.0 & 22 & A \\
\hline 39 & 336.0 & 169.0 & 338.0 & 169.0 & 22 & A \\
\hline 40 & 326.0 & 159.0 & 328.0 & 159.0 & 25 & A \\
\hline 41 & 272.0 & 105.1 & 274.0 & 105.1 & 20 & A \\
\hline 42 & 288.0 & 121.1 & 290.0 & 121.1 & 17 & A \\
\hline 43 & 274.0 & 107.1 & 276.0 & 107.1 & 20 & A \\
\hline
\end{tabular}




\begin{tabular}{|c|c|c|c|c|c|c|}
\hline 44 & 276.0 & 109.1 & 278.0 & 109.1 & 22 & A \\
\hline 45 & 276.0 & 109.1 & 276.0 & 258.0 & 20 & A \\
\hline 46 & 276.0 & 109.1 & 278.0 & 109.1 & 22 & A \\
\hline 47 & 312.0 & 145.0 & 312.0 & 294.0 & 18 & $\mathrm{~B}$ \\
\hline 48 & 308.0 & 180.0 & 310.0 & 182.0 & 17 & A \\
\hline 49 & 259.0 & 92.1 & 261.0 & 92.1 & 20 & B \\
\hline 50 & 259.0 & 92.1 & 261.0 & 92.1 & 35 & A \\
\hline 51 & 259.0 & 107.1 & 261.0 & 107.1 & 32 & B \\
\hline 52 & 248.0 & 180.0 & 250.0 & 182.0 & 18 & B \\
\hline 53 & 264.0 & 180.0 & 266.0 & 182.0 & 17 & A \\
\hline
\end{tabular}

\subsection{Plasma Stability Assay}

One major VSe technology application is OVt enhancement, thus making the lead compound ultimately intended for in vivo use. One desirable property of this class of compounds is reasonable stability in the physiological environments it will experience from the point of administration to the target cancer cells. The bloodstream contains various detoxifying enzymes for the purposes of removing foreign compounds. The simplest model of the bloodstream is blood plasma. It contains the aqueous and protein components of blood but cells have been removed. It was previously determined that VSel possessed poor stability in aqueous media leading to the hypothesis that it would be even more so in blood. New lead VSes need to have improved blood stability; therefore it became a priority to perform hit expansion and screen the library for compounds with improved stability and suitability for in vivo administration [68].

As adapted from Kerns and Di [68], $70 \mu \mathrm{M}$ stock solutions of each VSe were prepared in $\mathrm{MeOH}$ and mixed with Balb/c mouse plasma that was buffered 1:1 with PBS $(\mathrm{pH}=7.4)$ and cleared of particulate by centrifugation at $3000 \mathrm{rpm}$ for 15 mins. VSes were added to the plasma to a final concentration of $10 \mu \mathrm{M}$ in a total volume of $400 \mu \mathrm{L}$ 
in triplicate. Immediately upon mixing, $200 \mu \mathrm{L}$ of the mixture was quenched with $300 \mu \mathrm{L}$ of $5 \% \mathrm{FAw}$ to prevent further VSe degradation. The remainder of the mixture was incubated at $37^{\circ} \mathrm{C}$ for $3 \mathrm{hrs}$ and quenched in a similar manner. The quenched samples were spin filtered and the VSes were quantified by MRM AUP analysis. This allowed for the compounds' plasma stability to be calculated as a percentage of the compound detected $3 \mathrm{hrs}$ after the plasma incubation step relative to the original amount.

It needed to be ensured that VSes were not undergoing further degradation between mixing with plasma and LC-MS analysis. It was previously determined that acidic $\mathrm{pH}$ improves VSe stability. To validate that the 5\% FAw solution had successfully quenched the plasma-induced degradation, some of the VSe batches were analyzed $24 \mathrm{hrs}$ after quenching and showed that an insignificant amount of decomposition had occurred during that long period of time (Figure 4.5). An acidic environment reduces the concentration of hydroxide ions that are capable of nucleophilic addition at the $\beta$-carbon.

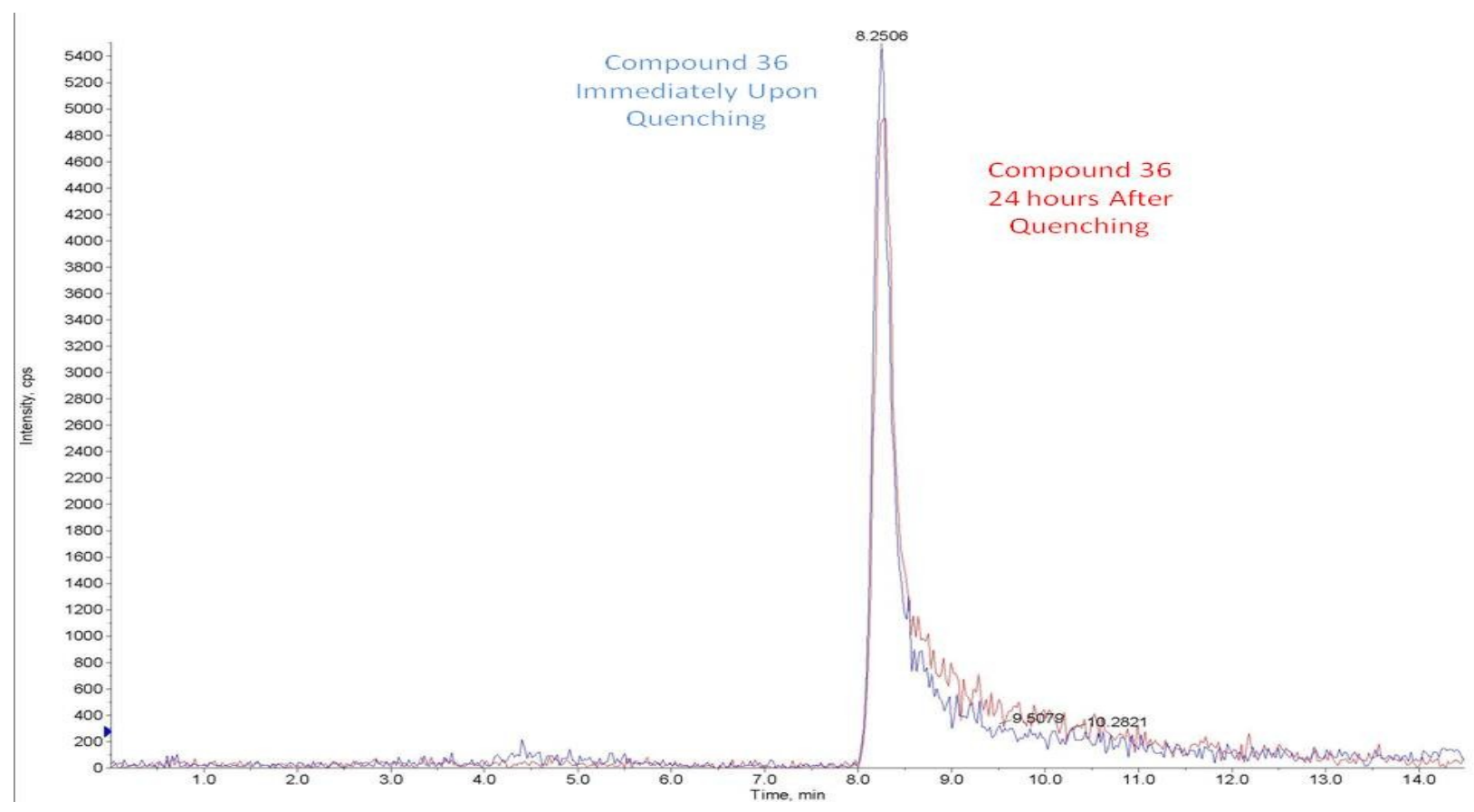

Figure 4.5: Quenching VSes with 5\% FAw was effective in limiting further degradation in plasma 
VSe degradation was observable when MRM traces of the same compound were overlaid before and after their incubation in plasma. As shown in Figure 4.6, the three VSe elution profiles do not co-elute and compound 37 showed the least amount of degradation from the 0 min time point (blue trace) to the $3 \mathrm{hr}$ time point (red trace). Meanwhile Compound 50 degraded at a faster rate by comparing the height of the grey chromatogram $(0 \mathrm{hr})$ to the green traces $(3 \mathrm{hr})$.

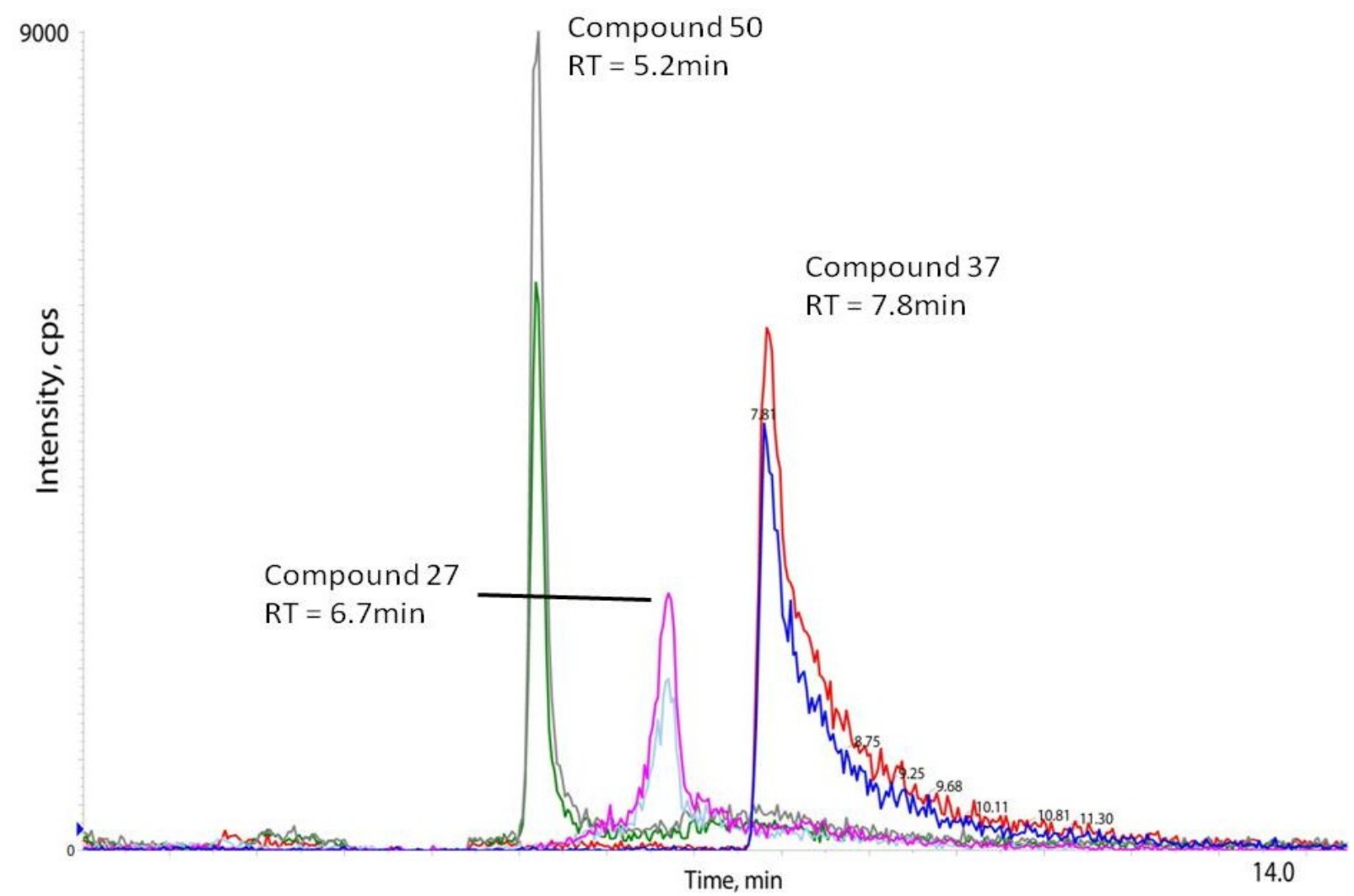

Figure 4.6: Observing the plasma-induced degradation of three VSes simultaneously after a $3 \mathrm{hr}$ incubation

The plasma stabilities for each VSe are displayed in Tables 4.2, 4.3 and 4.4 in addition to their structures and other properties that were investigated during the library screen. These other properties include activity (determined by Ramya Krishnan), toxicity (determined by Ramya Krishnan) and glutathione stability (determined by Mark Dornan). 
In short, the viral sensitization factor (activity) was determined by titering VSV $\Delta 51$ expressing Firefly luciferase (Fluc) in Vero cells 40 hrs post-infection using a Fluc reporter assay and each compound's activity was standardized relative to VSe1 over a wide range of concentrations [35]. The concentration providing the greatest enhancement in viral titer is indicated beside the activity in brackets. VSe cytotoxicity in the presence and absence of VSV $\Delta 51$ was measured with an alamarBlue metabolic dye and expressed as the lethal dose 50 (LD50) [35]. Glutathione stability was monitored by mixing $20 \mu \mathrm{M}$ VSe with $100 \mu \mathrm{M} \mathrm{GSH}$ at $37^{\circ} \mathrm{C}$ and monitoring the formation of the GSH adduct using an LC-MS equipped with a UV-Vis detector.

VSe1 possessed poor plasma stability ( $0 \%$ remained after a $3 \mathrm{hr}$ incubation), quickly conjugated to GSH (half-life $<5$ mins) and was poorly tolerated in combination with the virus $(\operatorname{LD} 50=17 \mu \mathrm{M})$. Despite characteristics that make it unsuitable for in vivo applications such as $\mathrm{OVt}$, it possessed strong activity that provided rationale to investigate new ring scaffolds with potentially improved properties. In Table 4.2, the broad diversification of the VSe1-based (compound 1) scaffold was studied. Substituting the $\beta-\mathrm{Cl}$ with an alkyl amine abolished VSe activity (compounds 3 and 4) and replacing both $\mathrm{Cl}$ with $\mathrm{Br}$ caused a significant decrease in activity while not improving GSH and plasma stability (compound 2). Substitution of the phenyl group (compounds 5, 6, and 7) did not fix the stability issues either. Switching to a 3,4-dichloro-5-hydroxy-1,5dihydropyrrol-2-one scaffold (compounds 9 and 10) retained some VSe activity. Even more promisingly was that compound 10 (MD01145) was more stable in plasma $(19.8 \%$ remaining after a $3 \mathrm{hr}$ incubation), reacted more slowly with GSH (half-life $=32 \mathrm{mins}$ ) 
and was more tolerated by 786-0 cells in the presence of virus. This was substantial reason to further investigate SAR using this scaffold.

Table 4.2: Diversifying the VSe1 ring scaffold in search of improved stability

\begin{tabular}{|c|c|c|c|c|c|c|}
\hline Compound & Structure & $\begin{array}{c}\text { Activity } \\
\text { (Peak Dose } \mu \mathrm{M})\end{array}$ & $\operatorname{LD50}(\mu \mathrm{M})$ & $\begin{array}{l}\text { LD50 with virus } \\
(\mu \mathrm{M})\end{array}$ & $\begin{array}{c}\text { GSH half-life } \\
(\mathrm{min})\end{array}$ & $\begin{array}{l}\text { Plasma stability } \\
\% \text { remaining at } 3 \text { hrs } \\
\end{array}$ \\
\hline 1 (VSe1) & & $1.00(60 \mu \mathrm{M})$ & 79 & 16 & $<5$ & 0 \\
\hline 2 & & $0.47(72 \mu \mathrm{M})$ & 87 & 50 & $<5$ & 0 \\
\hline 3 & & NE & ND & ND & NR & $65.6 \pm 6.5$ \\
\hline 4 & & NE & ND & ND & NR & 0 \\
\hline 5 & & $0.47(36 \mu \mathrm{M})$ & 41 & 27 & $<5$ & 0 \\
\hline 6 & & $0.31(60 \mu \mathrm{M})$ & 73 & 51 & $<5$ & 0 \\
\hline 7 & & $0.07(60 \mu \mathrm{M})$ & 52 & 17 & $<5$ & 0 \\
\hline 8 & & NE & $>90$ & $>90$ & $N R$ & $88.3 \pm 9.3$ \\
\hline 9 & & $0.49(120 \mu \mathrm{M})$ & 148 & 87 & 117 & 0 \\
\hline 10 (MD01 & & $0.30(60 \mu \mathrm{M})$ & 67 & 51 & 32 & $19.8 \pm 0.4$ \\
\hline
\end{tabular}

$\mathrm{NE}=$ no enhancement (inactive compound) $\mathrm{ND}=$ not determined, $\mathrm{NR}=$ no reaction with $\mathrm{GSH}$

To demonstrate more clearly the improvement in plasma stability between compounds 1 and 10, the plasma stability assay was repeated for these two compounds and quenched at multiple shorter time points in experimental triplicate (Figure 4.7). VSe1 
had a plasma half-life of 4 mins while MD01145 had an improved plasma half-life of 42

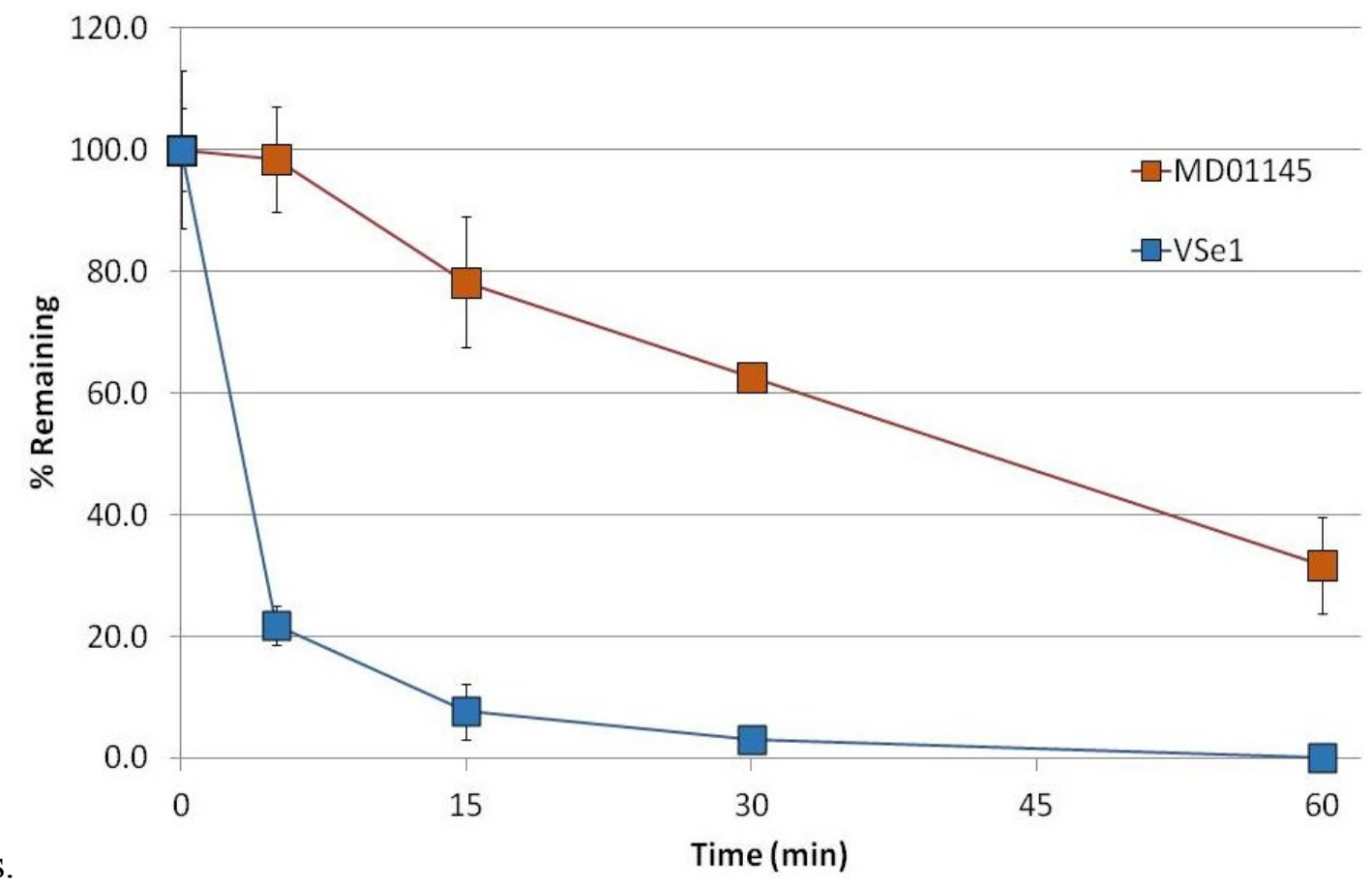

Figure 4.7: Time-course degradation of VSe1 and MD01145 in plasma

In Table 4.3, modifications made to the hydroxyl group (compounds 11-15) and the dichloro moiety (compounds 16-21) abolished VSe activity despite improved plasma stabilities. Modifications at the amine were investigated because the other functional groups (dichloro alkene, hydroxyl and amide) were necessary for VSe activity (Figure 4.8). This prompted the synthesis of numerous MD01145 analogues with substitutions made to the R-group extending from the amino nitrogen. 
Table 4.3: Exploring the importance of each MD01145 functional group

\begin{tabular}{|c|c|c|c|c|c|c|}
\hline Compound & Structure & $\begin{array}{c}\text { Activity } \\
\text { (peak dose } \mu \mathrm{M} \text { ) }\end{array}$ & LD50 $(\mu \mathrm{M})$ & $\begin{array}{l}\text { LD50 with } \\
\text { virus }(\mu \mathrm{M})\end{array}$ & $\begin{array}{c}\text { GSH half-life } \\
\text { (min) }\end{array}$ & $\begin{array}{l}\text { Plasma stablity } \\
\% \text { remaining at } 3 \mathrm{hrs} \\
\end{array}$ \\
\hline 11 & & NE & 269 & 182 & 64 & $42.5 \pm 9.6$ \\
\hline 12 & & NE & 206 & 203 & 118 & $47.6 \pm 1.4$ \\
\hline 13 & & NE & 63 & 39 & 21 & 0 \\
\hline 14 & & $0.03(17.8 \mu \mathrm{M})$ & 104 & 98 & $<5$ & $70.2 \pm 8.4$ \\
\hline 15 & & NE & 66 & 66 & 340 & $14.9 \pm 7.1$ \\
\hline 16 & & NE & $>360$ & $>360$ & ND & $98.2 \pm 3.7$ \\
\hline 17 & & NE & $>360$ & $>360$ & ND & $82.0 \pm 10.2$ \\
\hline 18 & & NE & $>360$ & $>360$ & ND & ND \\
\hline 19 & & NE & ND & ND & ND & ND \\
\hline 20 & & NE & $>360$ & $>360$ & NR & $102.9 \pm 1.6$ \\
\hline 21 & & NE & 287 & ND & NR & $102.7 \pm 10.8$ \\
\hline
\end{tabular}

$\mathrm{NE}=$ no enhancement (inactive compound), $\mathrm{ND}=$ not determined, $\mathrm{NR}=$ no reaction with $\mathrm{GSH}$

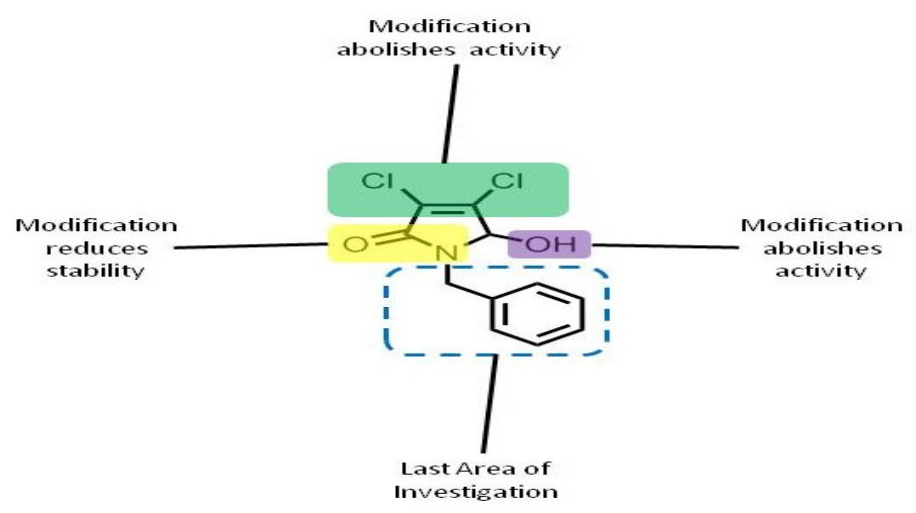

Figure 4.8: Summarizing the significance of each MD01145 functional group 
Highlights of the MD01145 analogue screen (Table 4.4) include the investigation of alkyl chain length and terminal unsaturation (compounds 22-27) which lead to the identification of compound 24 (MD03182) with a propyl R-group that showed remarkable stability in plasma (91.6\%) with one third retained activity. Cyclization of the R-group to a cyclopropyl group (compound 25, MD03013) had improved activity (0.53) that came at a reduction in plasma stability (54.8\%). Compound 28's R-group (MD03011) consisting of a morpholine ring demonstrated comparable activity in addition to improved stability relative to MD01145. Extending the length of the alkyl chain linking the phenyl group to the VSe ring scaffold revealed that a propyl chain (KK01007, compound 30) provided optimal stability. This prompted the diversification of the aromatic group with the addition of branches and unsaturations (compounds 32-36, 48) that abolished small molecule activity. The additions of hetero-atoms also lead to substantial losses in activity with the exception of compound 40 (MD03017), a paratrifluoromethylbenzene R-group. MD03017 was the only VSe to exhibit more activity than VSe1 while possessing a stability comparable to MD01145. The disadvantage with this compound is the raised toxicity both the absence and presence of VSV $\Delta 51$ (LD50 $=$ $13 \mu \mathrm{m})$, in comparison to VSe1 (LD50 $=17 \mu \mathrm{M})$. 
Table 4.4: Diversifying the R-group extending from the MD01145 amide

\begin{tabular}{|c|c|c|c|c|c|c|}
\hline Compound & $\mathrm{R}^{1}$ & $\begin{array}{c}\text { Activity } \\
\text { (Peak Dose } \mu \mathrm{M})\end{array}$ & LD50 $(\mu \mathrm{M})$ & $\begin{array}{l}\text { LD50 with virus } \\
\qquad(\mu \mathrm{M})\end{array}$ & $\begin{array}{l}\text { GSH half-life } \\
\text { (min) }\end{array}$ & $\begin{array}{c}\text { Plasma stability } \\
\% \text { remaining at } 3 \mathrm{hrs} \\
\end{array}$ \\
\hline 22 & in & $0.06(80 \mu \mathrm{M})$ & 125 & 58 & 68 & $72.0 \pm 3.0$ \\
\hline 23 & & $0.10(72 \mu \mathrm{M})$ & $>90$ & 55 & NR & ND \\
\hline 24 (MD03182) & & $0.34(120 \mu \mathrm{M})$ & 174 & 96 & 61 & $91.6 \pm 5.2$ \\
\hline 25 (MD03013) & & $0.53(80 \mu \mathrm{M})$ & 127 & 51 & 53 & $54.8 \pm 3.6$ \\
\hline 26 & & $0.26(96 \mu \mathrm{M})$ & 110 & 66 & 46 & $64.8 \pm 7.7$ \\
\hline 27 & & $0.04(80 \mu \mathrm{M})$ & 100 & 60 & 21 & $9.0 \pm 1.4$ \\
\hline 28 (MD03011) & & $0.67(80 \mu \mathrm{M})$ & 153 & 55 & 96 & $38.9 \pm 5.2$ \\
\hline 29 & & $0.39(72 \mu \mathrm{M})$ & 74 & 27 & 74 & $57.6 \pm 6.6$ \\
\hline 30 (KK01007) & & $0.61(32 \mu \mathrm{M})$ & 36 & 20 & 50 & $42.9 \pm 7.2$ \\
\hline 31 & & $0.34(40 \mu \mathrm{M})$ & 40 & 34 & 72 & $40.1 \pm 9.8$ \\
\hline 32 & & $0.05(27 \mu \mathrm{M})$ & 28 & 5 & 24 & ND \\
\hline 33 & & $0.02(48 \mu \mathrm{M})$ & 18 & 12 & 24 & 0 \\
\hline 34 & & $0.04(72 \mu \mathrm{M})$ & 74 & 6 & 31 & $48.0 \pm 16.5$ \\
\hline 35 & & $0.02(180 \mu \mathrm{M})$ & 36 & 23 & 43 & $63.8 \pm 3.2$ \\
\hline
\end{tabular}


Table 4.4: Diversifying the R-group extending from the MD01145 amide (continued)

\begin{tabular}{|c|c|c|c|c|c|c|}
\hline Compound & $\mathrm{R}^{1}$ & $\begin{array}{c}\text { Activity } \\
(\text { Peak Dose } \mu \mathrm{M})\end{array}$ & LD50 $(\mu \mathrm{M})$ & $\begin{array}{l}\text { LD50 with } \\
\text { virus }(\mu \mathrm{M})\end{array}$ & $\begin{array}{l}\text { GSH half-life } \\
\text { (min) }\end{array}$ & $\begin{array}{c}\text { Plasma stability } \\
\% \text { remaning at } 3 \mathrm{hrs}\end{array}$ \\
\hline 36 & & $0.04(72 \mu \mathrm{M})$ & 74 & 58 & 34 & $28.2 \pm 2.6$ \\
\hline 37 & & $0.06(48 \mu \mathrm{M})$ & 58 & 38 & 41 & $0.7 \pm 0.1$ \\
\hline 38 & & $0.06(216 \mu \mathrm{M})$ & 218 & 107 & 32 & $25.7 \pm 2.9$ \\
\hline 39 & & $0.06(60 \mu \mathrm{M})$ & $>90$ & 25 & 34 & $41.4 \pm 5.4$ \\
\hline 40 (MD03017) & & $1.6(27 \mu \mathrm{M})$ & 36 & 13 & 32 & $15.3 \pm 2.5$ \\
\hline 41 & & $0.22(40 \mu \mathrm{M})$ & 39 & 30 & 35 & $51.4 \pm 8.2$ \\
\hline 42 & & $0.01(60 \mu \mathrm{M})$ & 55 & 17 & 40 & $49.1 \pm 12.4$ \\
\hline 43 & & $0.15(60 \mu \mathrm{M})$ & $>90$ & 45 & 69 & $58.3 \pm 0.6$ \\
\hline 44 & & $0.15(45-60 \mu \mathrm{M})$ & 63 & 39 & 31 & $45.9 \pm 8.1$ \\
\hline 45 & & $0.01(48 \mu \mathrm{M})$ & 43 & 37 & 31 & $54.2 \pm 4.2$ \\
\hline 46 & & $0.17(40 \mu \mathrm{M})$ & 42 & 36 & 32 & $23.1 \pm 0.8$ \\
\hline 47 & & $0.10(40 \mu \mathrm{M})$ & 36 & 35 & 35 & $22.7 \pm 8.4$ \\
\hline 48 & & $0.03(80 \mu \mathrm{M})$ & 35 & 17 & 14 & $36.5 \pm 7.6$ \\
\hline
\end{tabular}


Table 4.4: Diversifying the R-group extending from the MD01145 amide (continued)

Compound

$\mathrm{NE}=$ no enhancement (inactive compound), $\mathrm{ND}=$ not determined, $\mathrm{NR}=$ no reaction with $\mathrm{GSH}$

The library screen lead to the identification of new VSes that are more suitable for in vivo applications, but also provided new R-group scaffolds to synthesize future analogues and perform more detailed lead optimization studies. The plasma stability data was investigated in greater detail to develop guidelines for furthering improvement. In Table 4.5 the VSes are ranked in order of stability, regardless of activity, and coloured to facilitate the drawing of conclusions. 
Table 4.5: Ranking the VSe analogues in order of highest (green) to lowest (red) plasma stability

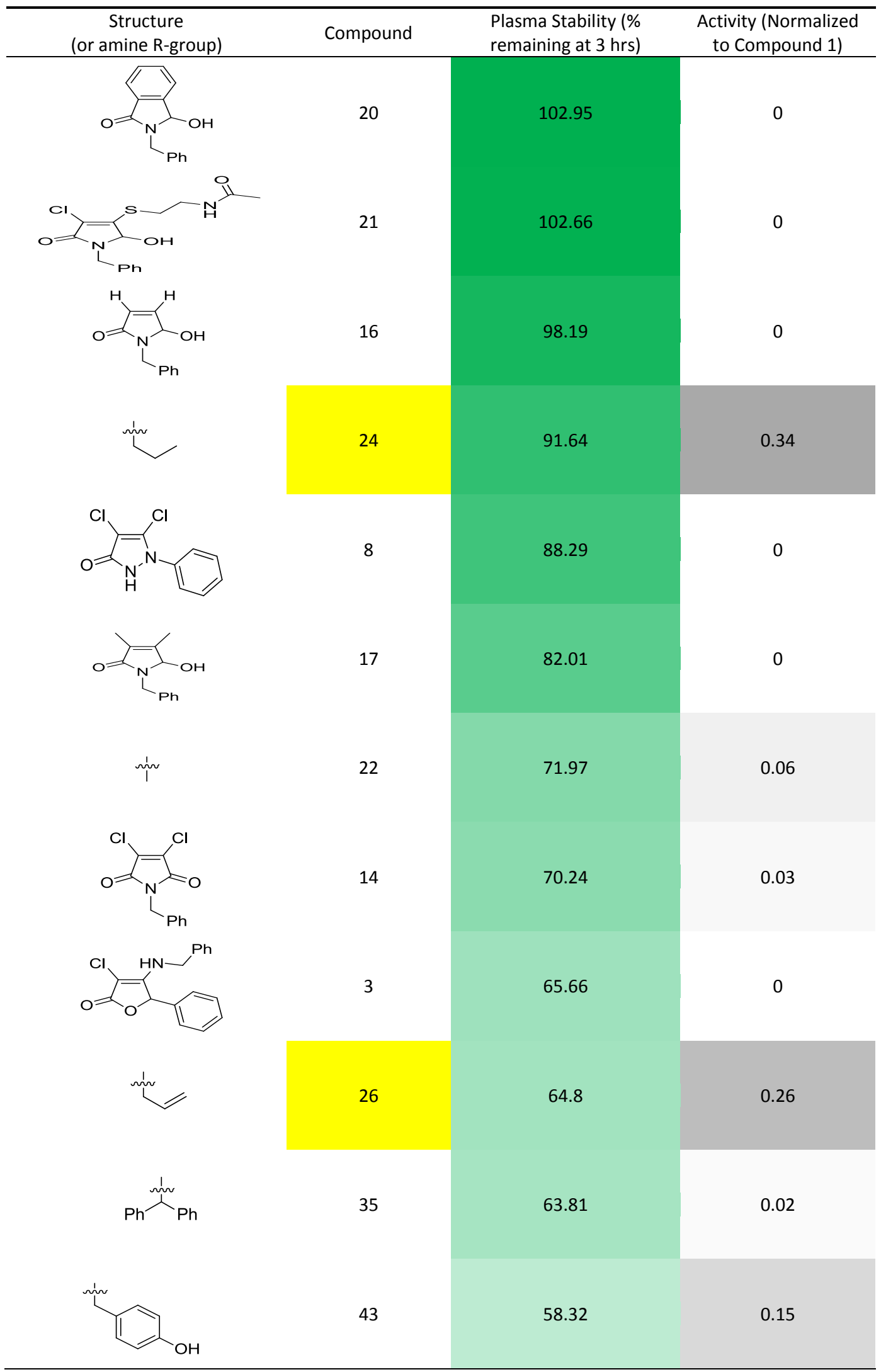




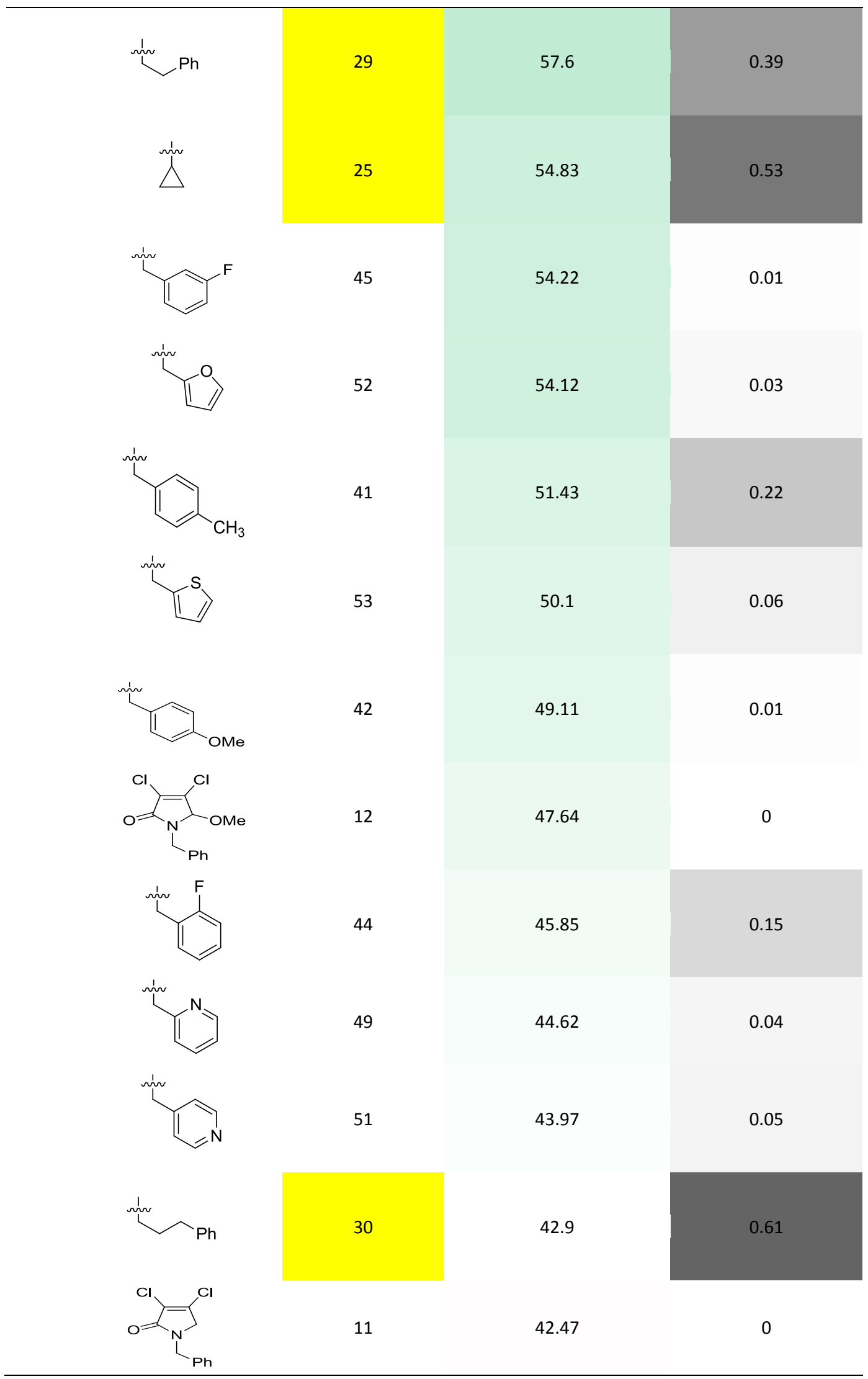




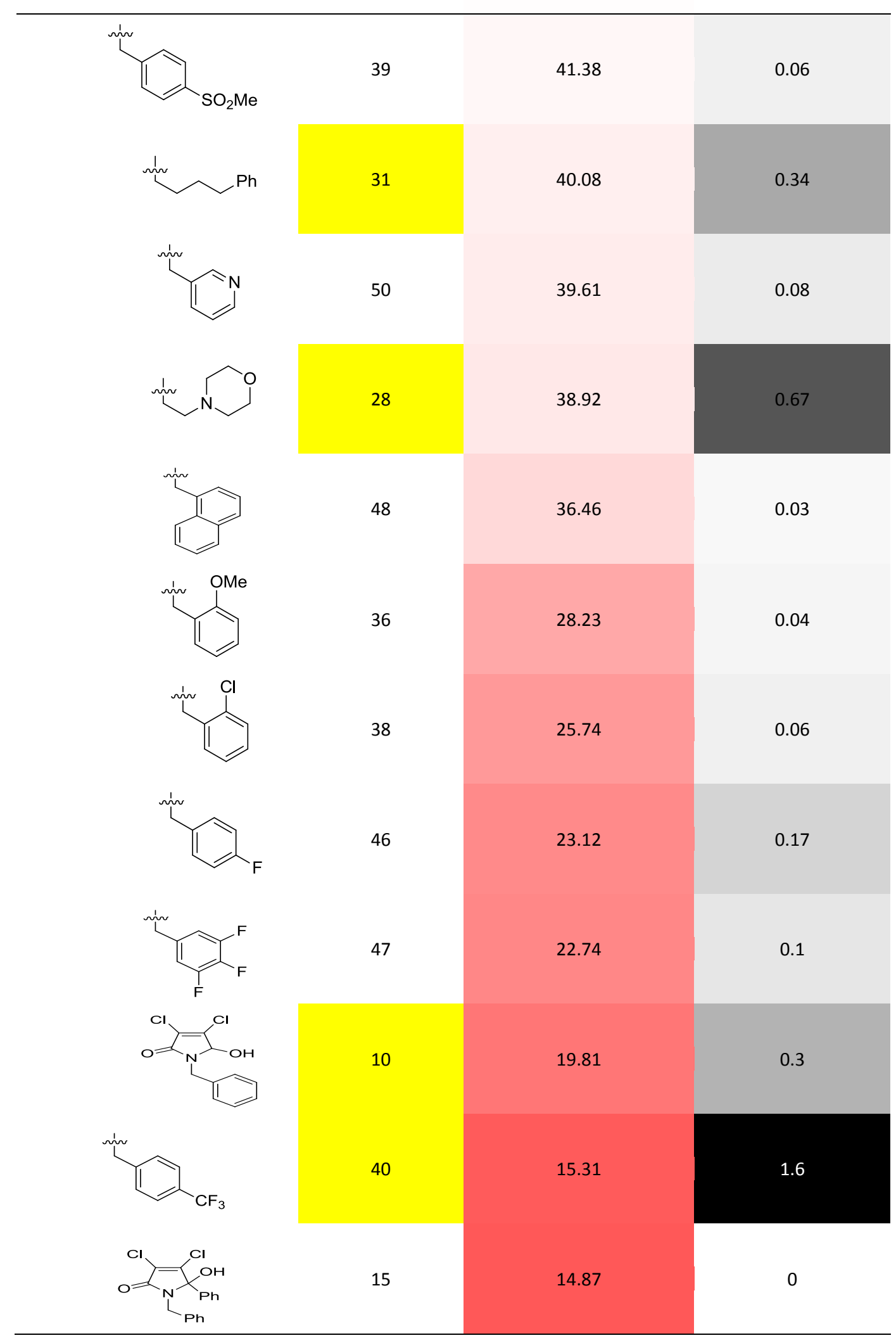




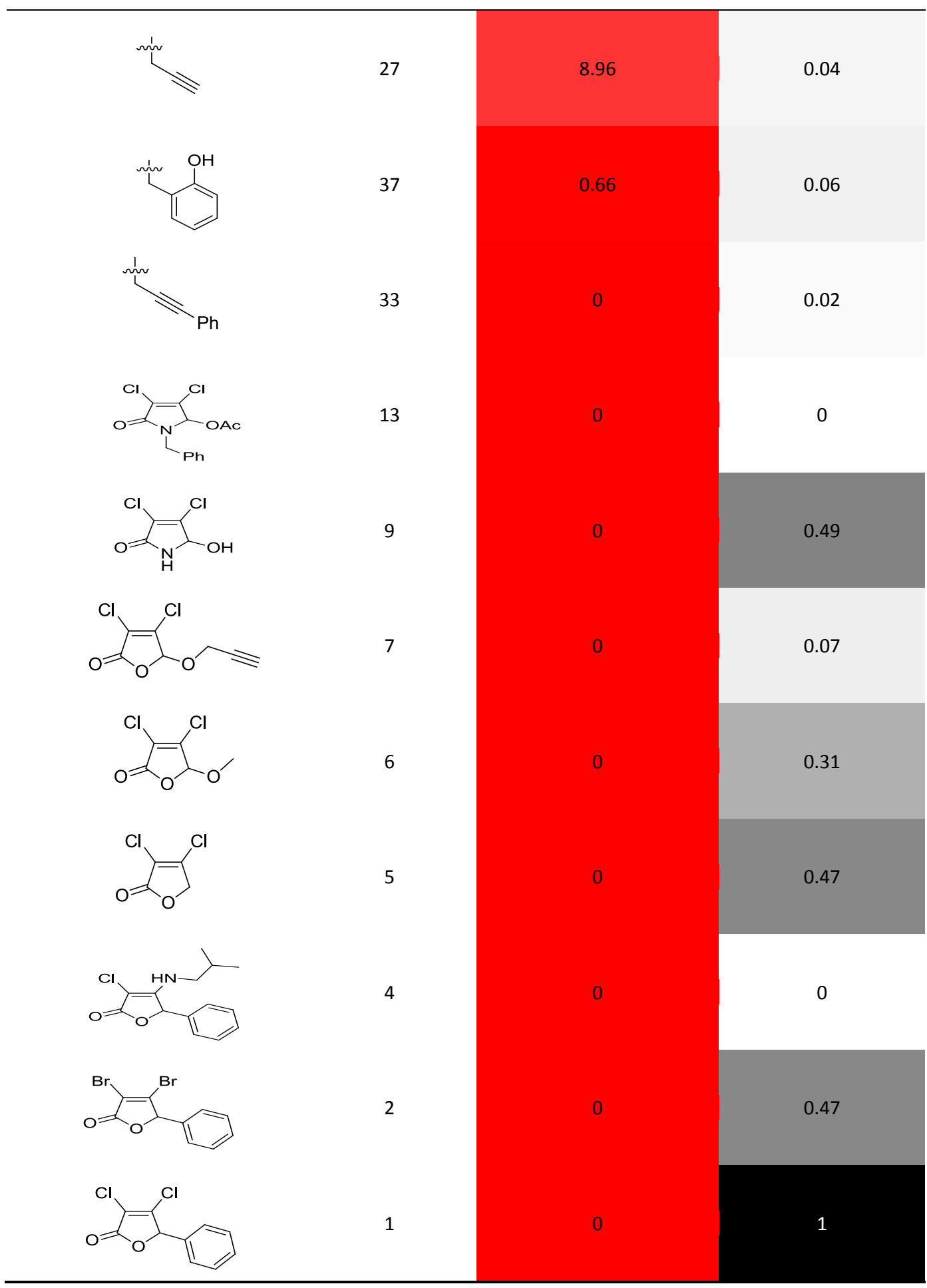

New lead candidates were highlighted in yellow as they possessed improved plasma stability while retaining an appreciable amount of VSe1's activity. From this table, trends that directly related structural properties to plasma stability were observed. 
Firstly, the removal of $\mathrm{Cl}$ heteroatoms lead to improved stability because the adjacent carbons are otherwise highly electrophilic and are available sites for nucleophilic attack. The addition of heteroatoms to the amide R-group also had an impact on stability (Table 4.6). Increasing the heteroatom content a greater distance from the amide predictably decreased stability as supported by Sawa et al. Antedrugs are often created by the addition of EWG's so that they are rapidly cleared from the body and reduce the harmful side effects [68]. As previously mentioned, the substitution of the furanone hemiacetal with a pyrrole-based scaffold significantly improved stability. This is supported by Breitenlechner et al. findings that amides can replace esters to improve stability while retaining activity during the optimization of azepane derivatives [68]. Thirdly, the addition of steric hindrance at the hydrolysable amide group increases stability as demonstrated by Brothwick et al [68]. This trend is reflected to an extent in the VSe data. Increasing the overall size of the R-group beyond a limit may decrease the local steric hindrance near the nitrogen. Extending the alkyl chain linking the pyrrole ring to the benzyl group on MD01145's structure demonstrates this phenomenon (Table 4.7). Additionally, overly large R-groups added to the amide nitrogen can introduce new hydrolysable sites that in turn decrease plasma stability. Terminal unsaturations in the Rgroup decrease stability due to the increased acidity of a terminal alkynyl hydrogren in comparison to an alkene, and more so an alkane (Table 4.8). 
Table 4.6: Altering the proximity and electronegativity of EW R-groups affects stability

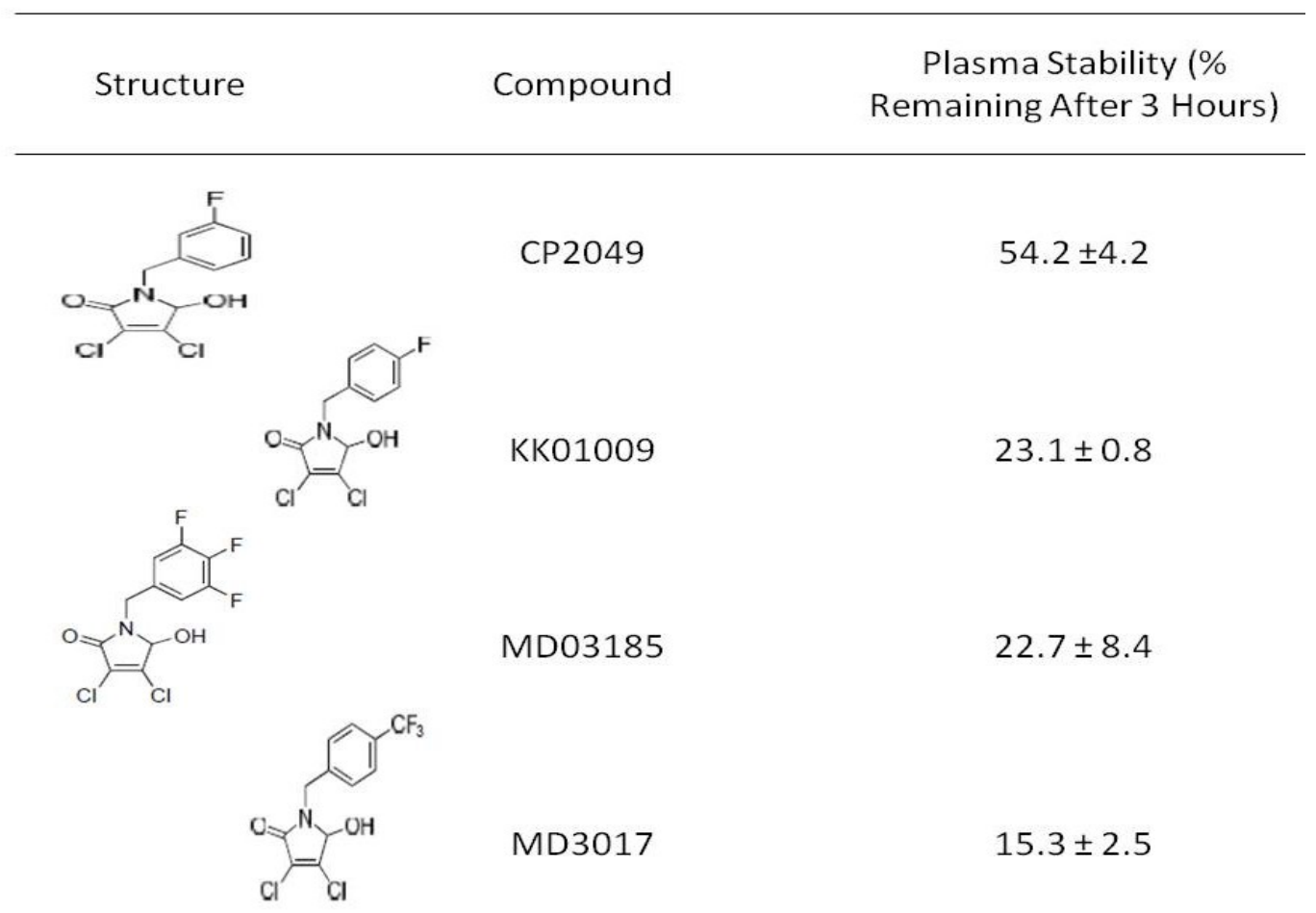

Table 4.7: Plasma stability is dependent on steric hindrance surrounding the hydrolysable amide

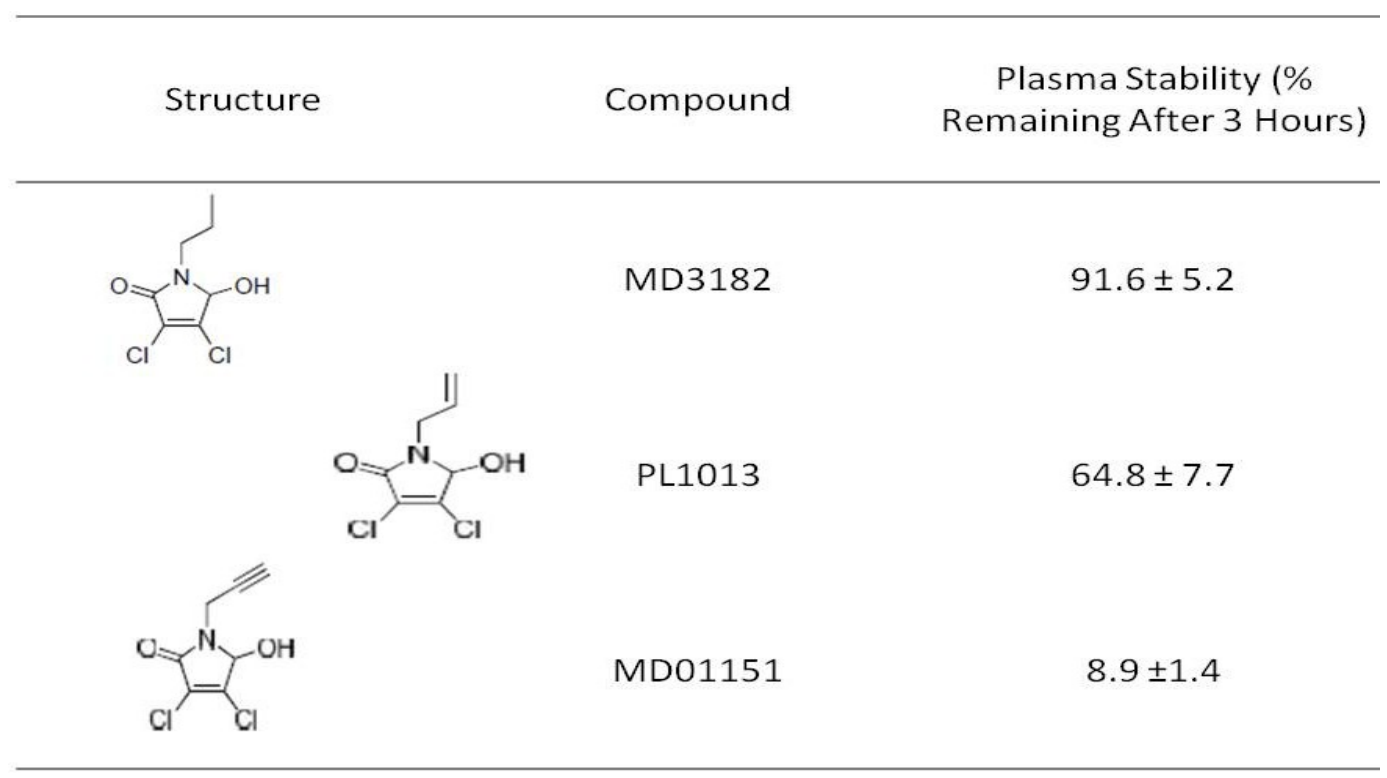


Table 4.8: R-group unsaturation leads to decreased plasma stability

\begin{tabular}{cc} 
Compound & $\begin{array}{c}\text { Plasma Stability (\% } \\
\text { Remaining After 3 Hours) }\end{array}$ \\
\hline &
\end{tabular}

Overall, these trends concerning can be applied to the synthesis of new leads with diversified R-groups in hopes of obtaining improved stability. Overall, the SAR library screen lead to the identification of VSes with improved properties for in vivo applications, such as OVt (Figure 4.9).

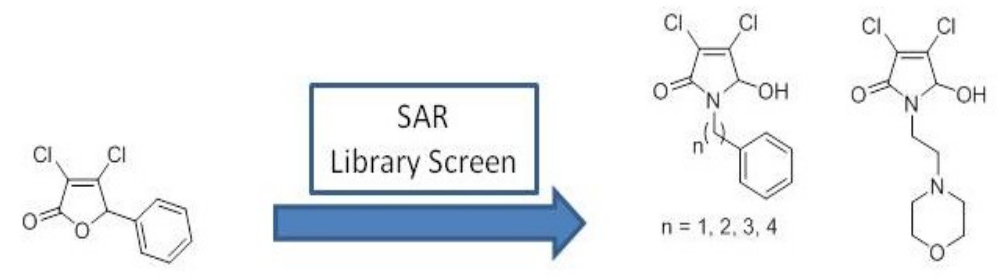

\section{$\underline{\text { VSe1 }}$}

- Active and tumour-selective

- Highly unstable: $\mathrm{t}_{1 / 2}$ plasma $<5 \mathrm{~min}$

- Poorly tolerated in vivo: $<10 \mathrm{mg} / \mathrm{kg}$
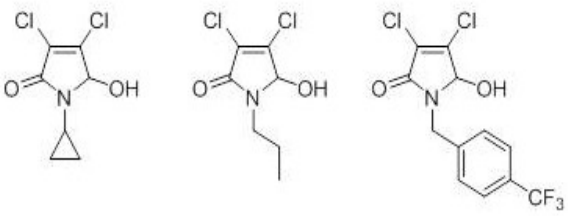

New Lead Compounds

- Retained activity and selectivity

- Increased stability: $\mathrm{t}_{1 / 2}$ plasma $15-57 \mathrm{~min}$

- Well tolerated in vivo: $>100 \mathrm{mg} / \mathrm{kg}$

Figure 4.9: Summarizing the findings of the VSe library screen including new lead compounds 


\subsection{Pharmacokinetics}

Lead VSes selected from the library screen were used in preliminary murine in vivo experiments to observe their behavior in a more complex biological system. Such studies can investigate the optimal route of VSe administration and dosage program to ensure drug efficacy, tumour selectivity and safety. VSe properties that can be assessed by in vivo studies that were previously not considered include: uptake and transport from the point of administration, permeability and solubility across various biological membranes

Pharmacokinetics (PK) is the study of a drug's fate after administration into a body to encompass drug absorption, bio-availability, bio-distribution and metabolism [68]. Compounds MD01145 and MD03182 were chosen for in vivo studies due to comparable normalized activities $(0.3)$ but dissimilar plasma stabilities $(19.8 \%$ and 91.3\% respectively). $200 \mathrm{mg} / \mathrm{mL}$ VSe stocks were prepared in DMSO and administered into Balb/c mice at a dose of $50 \mathrm{mg} / \mathrm{kg}$ in biological duplicate. The mice had previously been massed and implanted with VSV $\Delta 51$ resistant CT26 grafted colon cancer tumours of comparable size. The VSes were administered via two routes: intraperitoneal (IP) injection and intratumoural (IT) injection. Blood samples were collected from the saphenous vein at various time points and the blood was clotted by allowing the blood to incubate at room temperature for 15 mins. Serum (which does not contain cells or clotting factors) was collected after a centrifugation step at $14000 \mathrm{rpm}$ for 15 mins at $4^{\circ} \mathrm{C}$ and diluted 1:1 with PBS. The samples were spin filtered and the metabolites were collected for LC-MS analysis. The tumours were extracted and suspended in $500 \mu \mathrm{L}$ PBS

and homogenized for 30 mins at $30 \mathrm{~Hz}$ using a TissueLyser III (Retsch) homogenizer and 
two steel balls. Homogenates were spin filtered and the extracted metabolites were collected for LC-MS analysis. To prevent further VSe degradation, it was important to keep biological samples in the frozen state until analysis that was performed in technical triplicate.

Firstly, the intact compounds were relatively quantified by MRM. Serum samples obtained post-IP injection revealed that plasma stability does not necessarily translate into in vivo blood stability. Despite showing decreased plasma stability during the ex vivo VSe screen, MD01145 (32\% stable) proved to be more stable than MD03182 (0\%) over a $1 \mathrm{hr}$ metabolism period (Figure 4.10). MD01145's blood stability is mirrored by the plasma time-course stability in Figure 4.7. MD03182 was not detected 60 mins postinjection while only trace amounts were detected after 30 mins. MD03182 showed less MRM signal than MD01145 in the early time point, even though they were injected in an equimolar fashion. This is either due to an increased rate of degradation or in general, MD03182 provided less MRM intensity during the analysis of stocks prior to the plasma stability assay. Neither compound was detected in the samples at the 0 min time point as expected, since it took time for absorption, uptake and circulation to ensue. If MD01145's in vivo stability is extrapolated to the $3 \mathrm{hr}$ time-point, $10 \%$ of the original VSe amount would remain which is lower than the $19.8 \%$ stability observed in the ex vivo stability screen. This is a reasonable result when taking uptake of the drug, binding to blood cells or degradative enzymes that are not present in isolated plasma into consideration. 


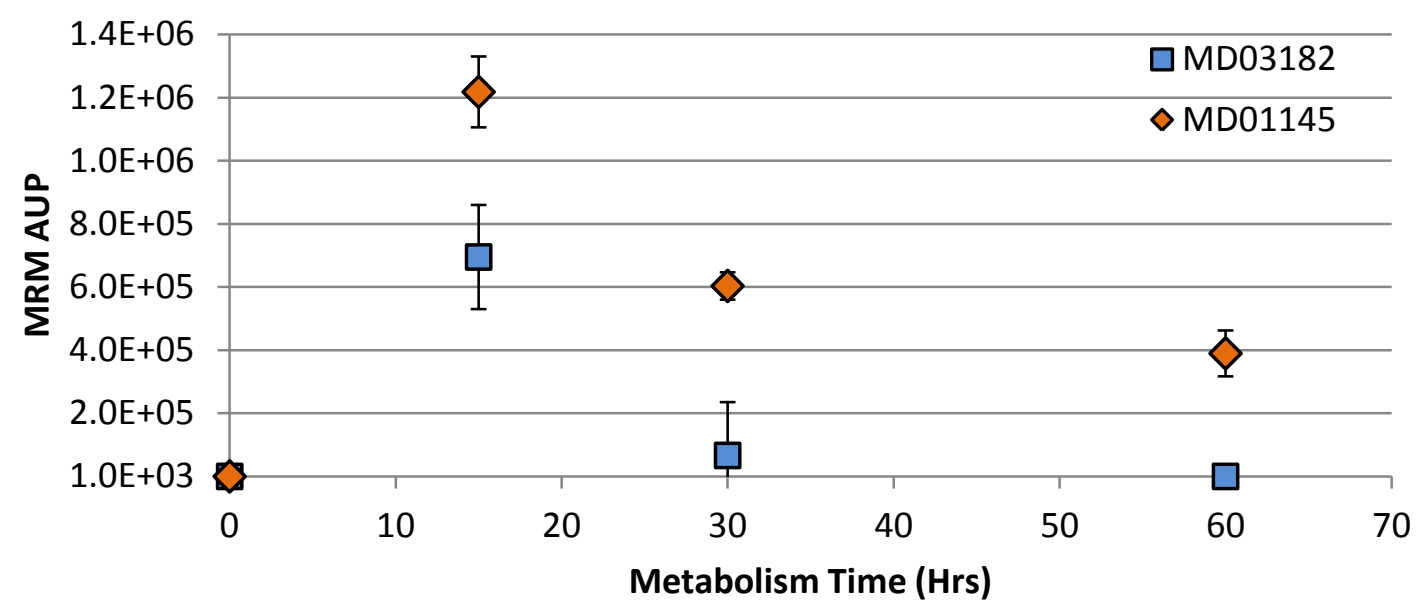

Figure 4.9: Relative quantitation of VSes from blood samples obtained from IP injection

The stability of MD03182 slightly improved in the tumoural environment following IT injection, but it still degraded faster than MD01145 (Figure 4.11). Trace amounts (1\%) of MD03182 were detected 3 hrs post-injection and only $4 \%$ was detected after $1 \mathrm{hr}$. MD01145's intratumoural stability (9\%) after $3 \mathrm{hrs}$ was comparable to its blood stability (10\%). Neither compound was detected in the 0 min time point samples that were collected prior to VSe injection to validate the MRM signal is VSe-specific and not from an isobaric metabolite.

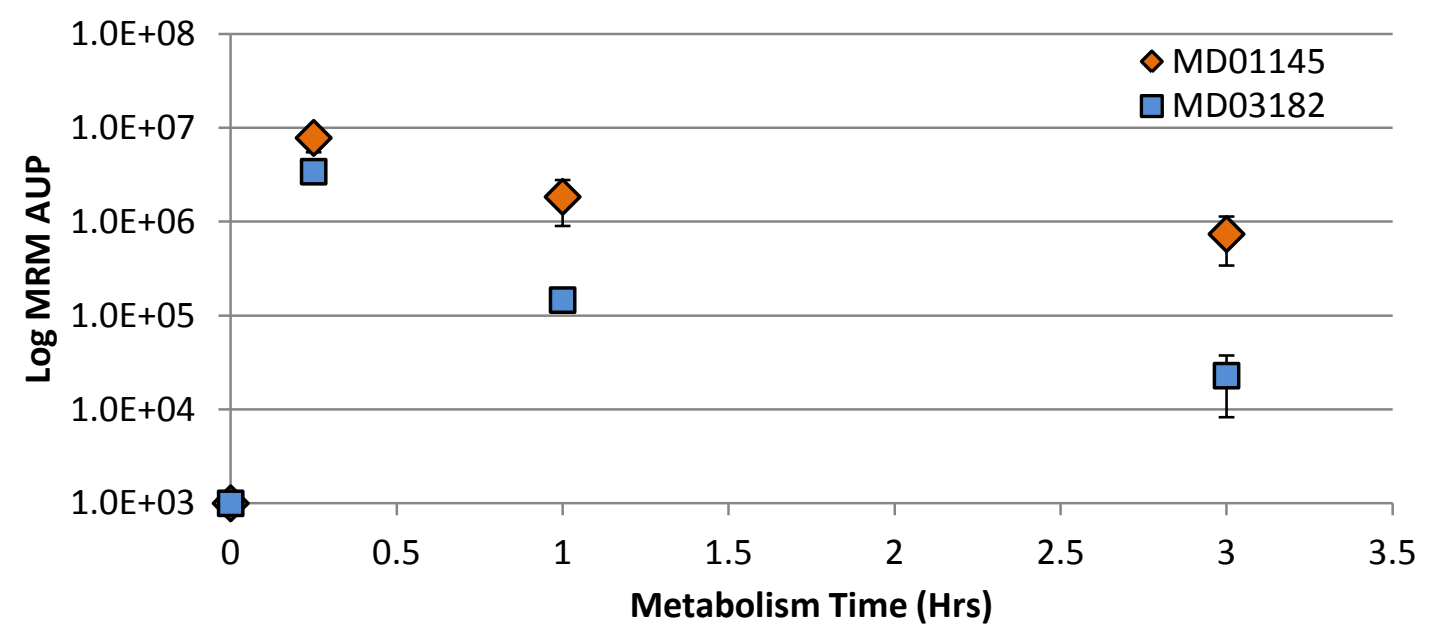

Figure 4.10: Relative quantitation of VSes from tumour samples obtained from IT injection 
It should be noted that neither compound was detected in the tumour homogenates when administered by IP injection. Similarly, only traces amounts of either compound were detected in the blood following IT injection. This indicates that VSes may have difficulties travelling across the tumoural membrane. Future experiments can address this potential obstacle using a tumour penetrance competition assay. Grafted tumours can be extracted and mixed ex vivo with a solution containing equimolar concentrations of three VSes. The VSes found at the highest abundance in tumour after incubation can be concluded to be the best at penetrating the target tissue.

\subsection{Metabolomics}

The PK studies revealed that VSes are degrading at a faster rate in vivo which brought their metabolic fate into question. Identified metabolites related to VSes can provide information about their intracellular target, unintended off-targets or their mode of excretion. EMS scans from PK samples were monitored for ions with a $33 \%$ or $66 \%$ $[\mathrm{M}+2]^{1+}$ isotope ratio; indicative of chlorinated compounds. Due to the low abundance of such compounds in vivo, it was assumed that such ions were VSe-specific. There were numerous ions with prominent $[\mathrm{M}+2]^{1+}$ isotopes but the majority were determined to not be VSe-specific because the relative intensity ratio was not near $33 \%$ or $66 \%$. Additional ions exhibiting $[\mathrm{M}+2]^{1+}$ isotopes that were also detected in the negative control samples were disregarded and assumed to be unrelated to the VSes. Neutral loss (NL) scans of 36 $\mathrm{Da}$ and $38 \mathrm{Da}$ were also applied since the loss of $\mathrm{HCl}$ would be expected for chlorinated metabolites. Two metabolites that overcame these criteria were the covalent additions of $271 \mathrm{Da}$ and $67 \mathrm{Da}$ to the intact VSe (Figure 4.12). The former was detected in the EMS 
scans prior to the elution of the intact VSe. The latter eluted after the intact VSe and was detected in both the EMS and NL scans $(B \& C)$ at the same retention time. Comparing the intensities of the ions in NL36 and NL38 scans showed the metabolite is singly chlorinated (33\% relative intensity), as could be concluded for the $[\mathrm{M}+271]^{1+}$ conjugate. Both metabolites were detected in both MD01145 and MD03182-containing samples at the appropriate $\mathrm{m} / \mathrm{z}$, thus validating their relation to VSes.
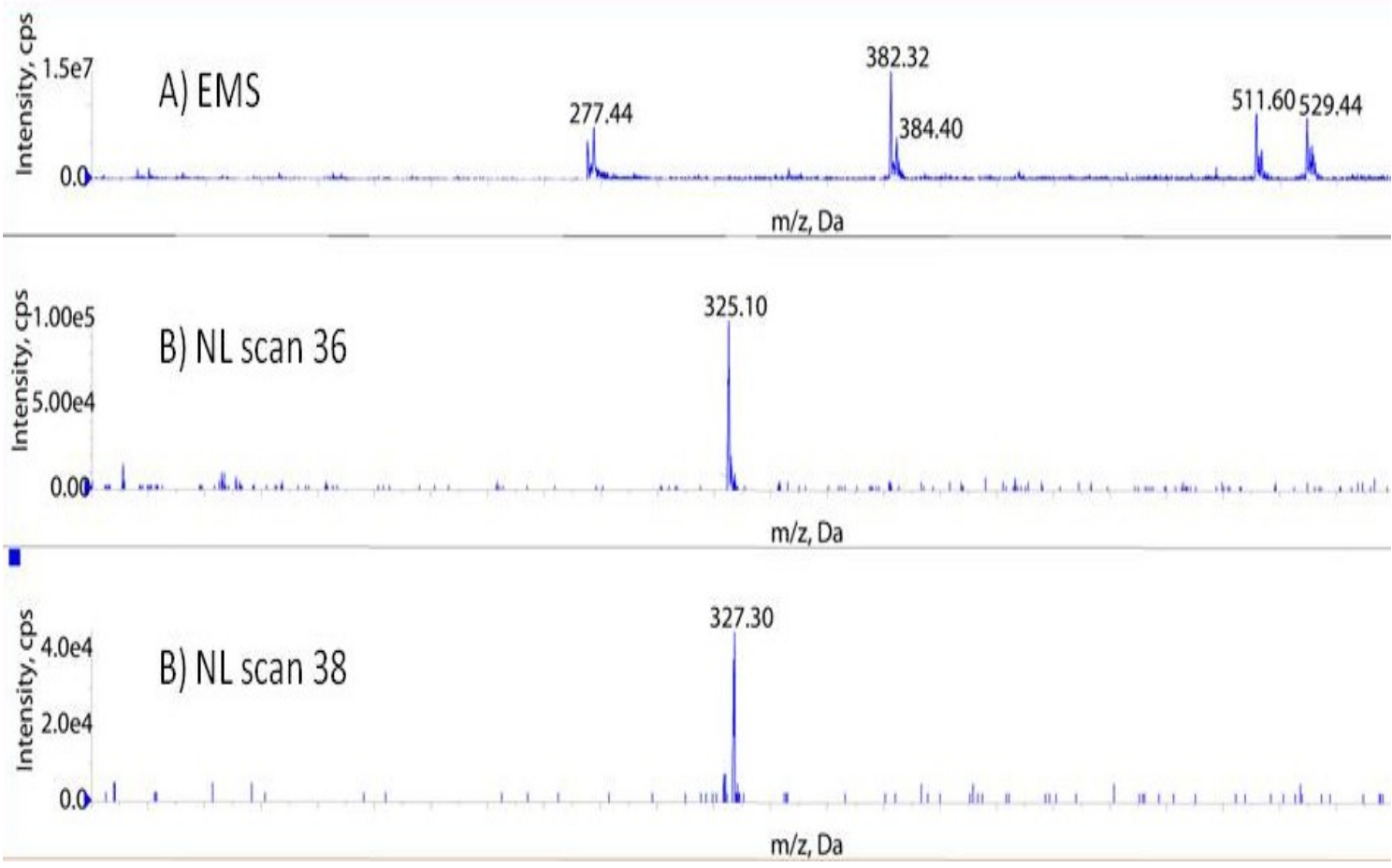

Figure 4.11: The detection of VSe-specific metabolites using EMS and NL scans

Nuclear magnetic resonance (NMR) would have facilitated the identification of these metabolites, but would have required large quantities of each metabolite with high purity. Ten MD01145-containing blood samples were separated by HPLC and the two metabolites were isolated using chromatograms from the PK samples to estimate elution points. The fractions were combined, dried using the Speed-vacuum and resuspended in $100 \mu \mathrm{L}$ of $2 \% \mathrm{ACN}$ in FAw. Using the 30 min sensitizer gradient on the QStar XL mass 
spectrometer, product ion scans of each metabolite were performed using the same optimal collision energy as MD01145 (25 eV).

A product ion scan of the $529 \mathrm{Th}$ ion revealed that MD01145 was losing one $\mathrm{Cl}$ atom and being replaced by glutathione (GSH) by nucleophilic addition with its cysteinyl sulfur. Glutathione is a highly abundant natural antioxidant that is responsible for detoxifying electrophilic compounds in phase II metabolism by making the oxygenreactive species more polar [69]. The identification of the MD01145-GSH adduct prompted the GSH stability assay in section 4.3.

A product ion scan of the 325 Th ion was not informative, but the detection of the 91 Th fragment further confirmed that the molecule was specific to MD01145 and the methyl benzene R-group is not modified by in vivo metabolism. Direct infusion accurate mass scans of the metabolite obtained a metabolite mass of $325.0694 \mathrm{Th}$. Assuming that the VSe was losing one $\mathrm{Cl}$ atom and being replaced by the metabolite in a similar fashion to the GSH adduct, the VSe gaining the addition of 103.0382 Da. The Metlin metabolite database was searched using the calculated mass and possible additions with nucleophilic functional groups were listed (Figure 4.13) [70].

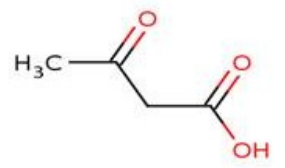

Acetoacetic acid

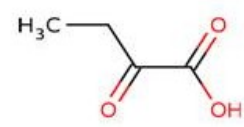

2-ketobutyric acid

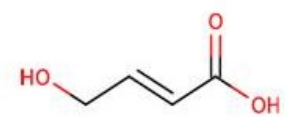

4-hydroxycrotonic acid

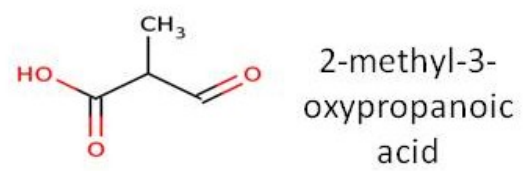

Figure 4.12: Possible metabolites conjugating to VSes based from accurate mass scans 
The metabolites identified by Metlin were within $7 \mathrm{ppm}$ of the experimental to provide confidence in the selected metabolites. Three compounds were regarded as unlikely VSe covalent additions due to their localizations. Acetoacetic acid is broken down in the liver to produce ketone bodies while succinic acid semialdehyde and 4hydroxycrotonic acid act as neurotransmitter analogues in the central nervous system. 2ketobutyric acid is linked to several types of cancer because it is implicated in upregulated amino acid (glycine, methionine, valine, leucine, serine and threonine) catalysis pathways [71]. 2-methyl-3-oxopropanoic acid is a product of the propanoate catabolic pathway which is also upregulated in kidney cancer [72]. Choosing between these two isomers would prove to be difficult by MS but could be facilitated with $\mathrm{MS}^{3}$. Although, functional groups that use oxygen for nucleophillic addition (as opposed to thiols or amines with more labile lone pairs of electrons) are questionable for this type of reaction.

The product ion scans of both GSH and the $103 \mathrm{Da}$ VSe adducts were used to develop MRM methods for the respective metabolites. Using the 4000 Qtrap MS, the metabolites were relatively quantified in the blood filtrates and tumour homogenates. The VSe-GSH metabolite accumulated over a short amount of time but was not detected beyond 30 mins post-injection (Figure 4.14), most likely due to subsequent phase III metabolism and excretion. An interesting result that needs to be confirmed with more biological replicates was that the VSe-GSH metabolite was not detected in tumour samples. The unidentified metabolite also formed quickly and accumulated over longer periods of time, particularly in the tumoural environment (Figure 4.15). 

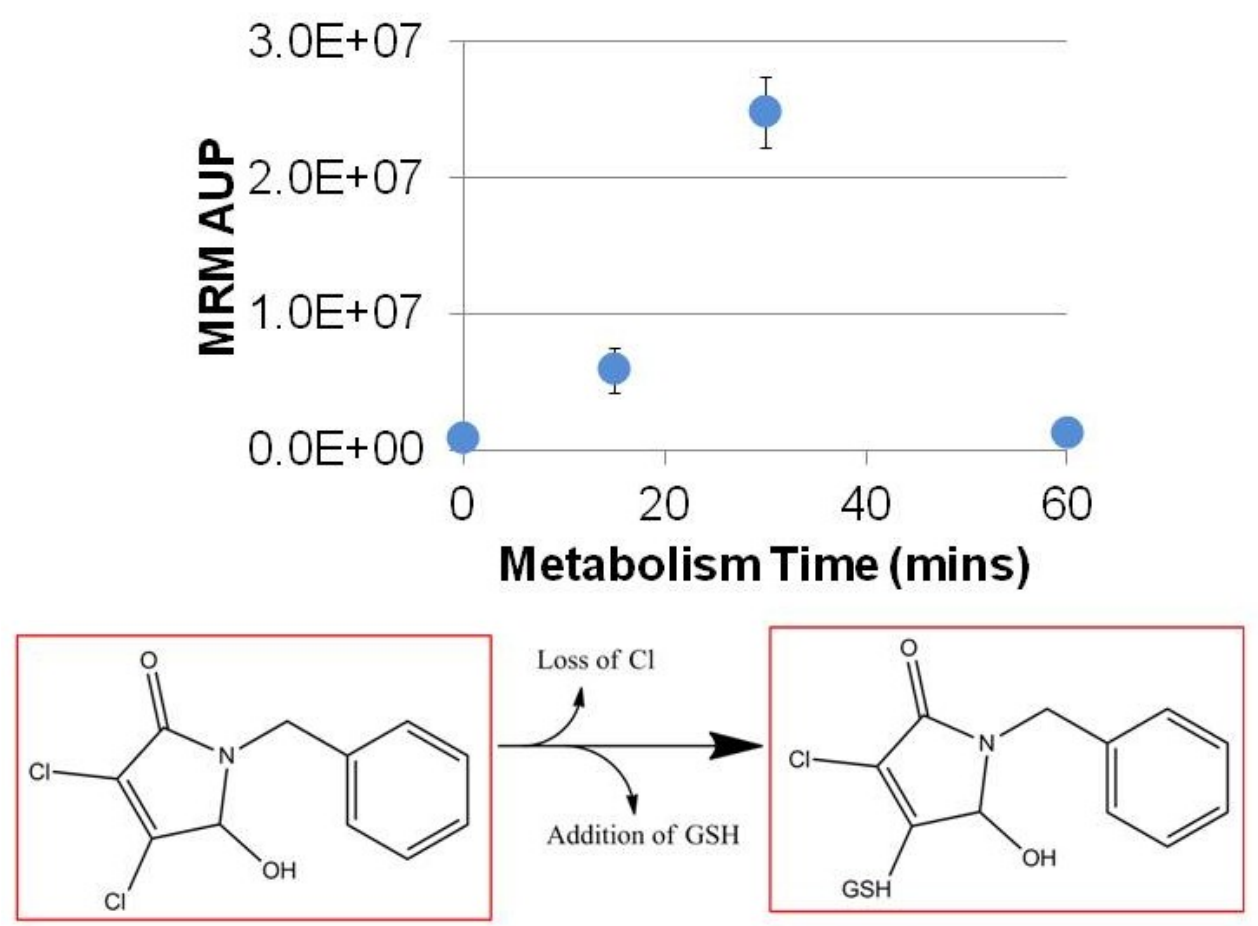

Figure 4.13: The VSe-GSH adduct forms quickly but is short-lived in blood
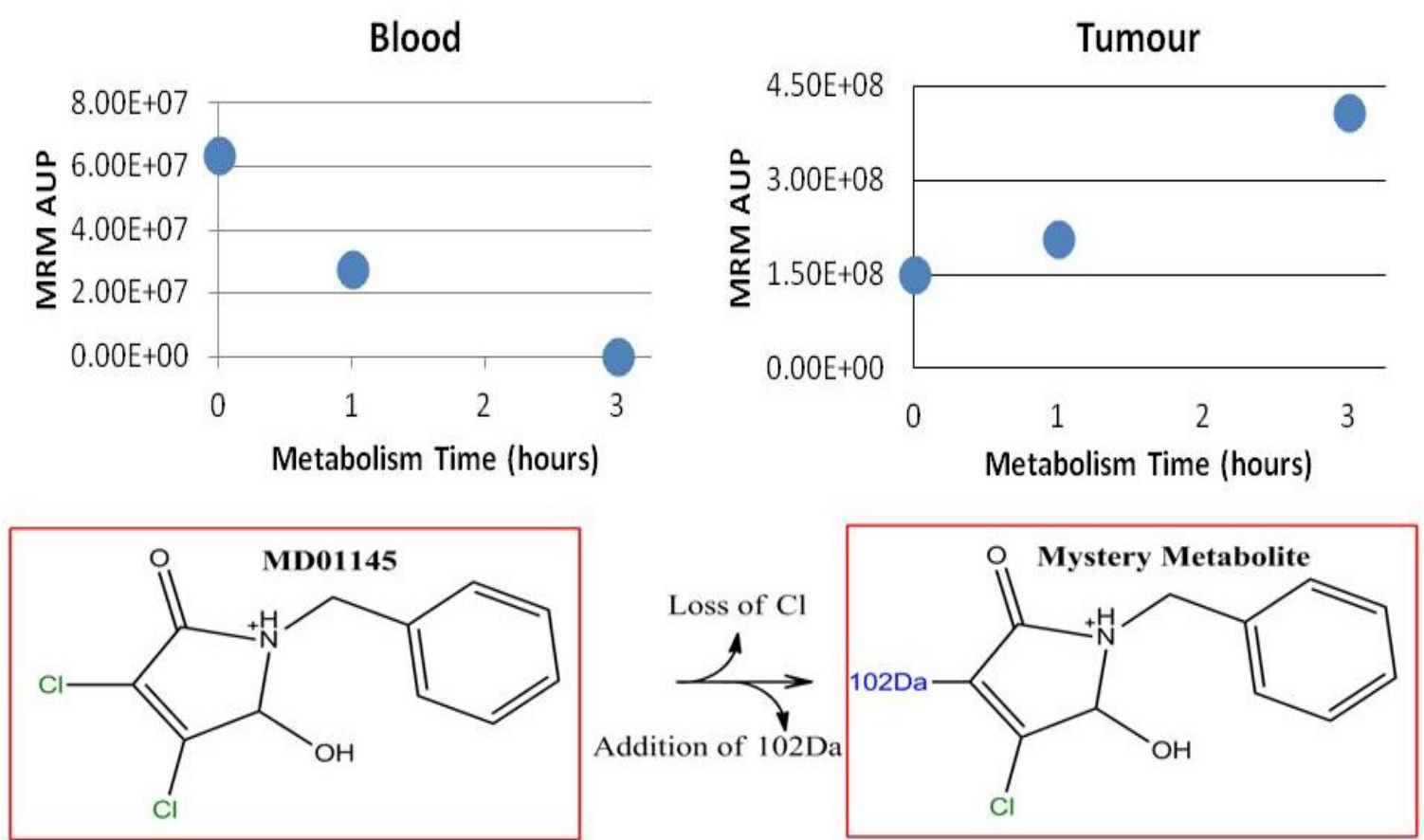

Figure 4.14: The unidentified metabolite accumulates in tumour tissues over time but degrades in blood 


\subsection{Conclusions}

In summary, mass spectrometry was a useful tool in screening a library of VSes to identify analogues with properties that were better suited for $\mathrm{OVt}$; namely plasma stability. MRM methods with optimized sensitivities were developed for each analogue using product ion scans by direct infusion. Structural trends were noticed that could lead to further the opitmization of lead compound R-groups. Monitoring VSe pharmacokinetics and metabolomics in murine models lead to the detection of two VSespecific metabolites. The in vivo studies also demonstrated the importance of testing tumour penetrance and whole blood stability in contrast to plasma stability. The next steps in this area of VSe research include reproducing the in vivo data and repeating the process with other lead compounds to see if stability and metabolomic trends are consistent. 


\section{Chapter: VSe proteomic studies}

\subsection{Drug target identification}

The approval of novel pharmaceuticals is becoming increasingly complicated as a result of more stringent safety measures. Detailed knowledge of drug-target partners is necessary to confidently state the mechanism through which drugs act but of equal importance is off-target characterization which cause poor drug activity or toxicity. The field of target identification has seen great advances in recent decades with the introduction of genomic-based experiments such as the yeast two-hybrid model and the protein microarray. These methods rely on target gene expression but do not directly address the protein target's activity responsible for the intended therapeutic effect in the biological system. The combination of SDS-PAGE and LC-MS is another breakthrough target identification technique but similarly depends on gene expression as opposed to activity. This limitation creates opportunities to develop alternative techniques [73].

Activity-based protein profiling (ABPP) is the specific study of individual protein activity in response to various stimuli, despite the tens of thousands of proteins that compose the proteome. ABPP has become the standard tool for target identification through the design of molecular probes that bind to the target's active-site. The probe's structure consists of a binding group, a reporter group and a linking group between the two. Once the binding group has covalently modified the target, the reporter group will enable detection of the targets; either intended or unintended. ABPP experiments also serve as a useful tool in assigning protein function in physiological or pathogenic pathways using probes based from naturally occurring products and substrates [73]. 
ABPP experiments are limited by the necessity of probe modification with retained activity. This restriction can be avoided using the drug affinity responsive target stability (DARTS) technique which is independent of the drug's mechanism. It is based on the principle that target proteins are less susceptible to proteolysis upon drug bindinginduced stabilization. This technique was used to reveal that the natural product revasterol targets the machinery associated with translation initiation [74].

Previously, the VSe mechanism of action (MOA) had not been clarified other than significantly reducing the expression of interferon-induced antiviral genes [34]. Knowledge of the MOA and the VSe intracellular interacting partner would be helpful in directing future experiments. This would include leading the synthesis of VSes with improved binding, and thus activity, using information from the target's active site. This chapter will discuss how DIGE and ABPP strategies were coupled with LC-MS to investigate the VSe MOA.

\subsection{Differential gel electrophoresis}

Isoelectric point $(\mathrm{pI})$ is the $\mathrm{pH}$ at which the overall charge of a protein is neutral and can be used to characterize proteins. A mixture of proteins can be separated by isoelectric focusing (IEF) which involves loading the proteins onto an immobilized $\mathrm{pH}$ gradient (IPG) on a polyacrylamide strip and exposed to an electrical current (Figure 5.1). Proteins migrate along the gradient towards the oppositely charged electrode until they reach their $\mathrm{pI}$ and cease to migrate. Migration beyond the $\mathrm{pI}$ causes the protein to switch polarities and return to the $\mathrm{pI}$ where they are focused into bands [75]. 


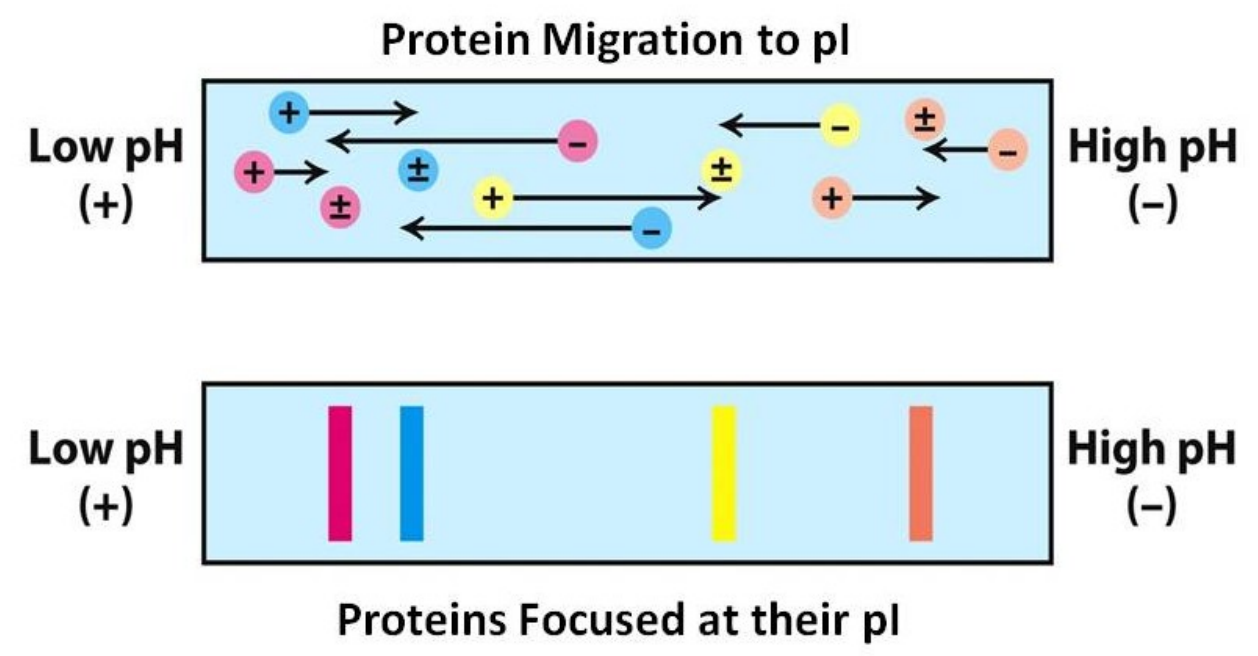

Figure 5.1: The migration of proteins to their PI along an IEF gradient. Adapted from [3]

IEF has limited applications as an individual technique but its potential is realized when combined with SDS-PAGE. After IEF, the IPG strip is loaded onto an SDS-PAGE gel and the proteins are separated in the other dimension by size to produce an array of spots representative of individual proteins. This tandem strategy termed 2-D SDS- PAGE enhances protein separation resolution relative to 1-D SDS-PAGE and reduces sample complexity to observe changes to the entire cell proteome. The comparison of proteomes using 2-D SDS-PAGE under different treatments is known as differential gel electrophoresis (DIGE) [75].

In DIGE experiments, protein spots at the same $\mathrm{pI}$ and molecular weight on different two-dimensional gels can be relatively quantified by the staining intensity. Proteome samples under different test conditions can be labeled with different coloured fluorophores and combined onto the same 2-D gel. The fluorescent signal detected at various excitatory wavelengths shows the abundances of individual protein in response to a test treatment [76]. An internal fluorescent standard avoids gel-to-gel variation. 


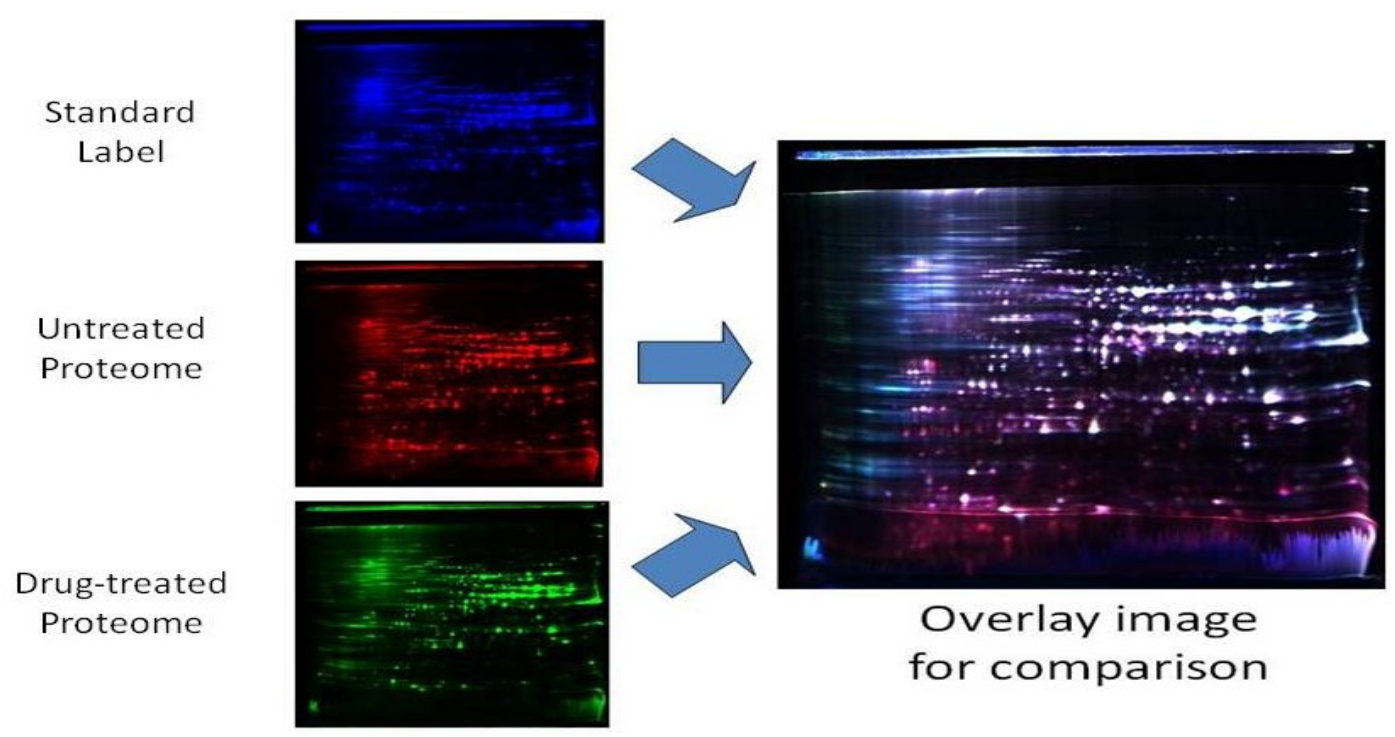

Figure 5.2: DIGE shows proteome expression changes upon drug treatment. Adapted from [4]

An increase in intensity upon pharmaceutical treatment signifies the small molecule has inhibited the protein or an interacting metabolic pathway. Negative feedback regulation of cellular homeostatic conditions dictates that gene expression will be enhanced to counteract the loss in activity. Oppositely, if a protein spot decreases in intensity the small molecule is amplifying the activity of the protein's pathway and gene expression is reduced to maintain equilibrium. One limitation of this method is it must be ensured that proteome samples are equimolar, but protein quantitation strategies are highly variable. DIGE has great potential in the identification of novel biomarkers for diseases such as cancer [77] and Alzheimer's [78]. It also gives clues into the pathways through which pharmaceuticals act as shown by Wierzba et al. that the cancer therapeutic geldanamycin is an inhibitor of heat-shock protein 90 [79]. This strategy was therefore applicable to VSe proteomic studies. 


\subsection{3 DIGE analysis of the non-cancerous cell proteome}

Human embryonic kidney (HEK293) immortalized cells were cultured in $10 \mathrm{~cm}$ culture dishes with Dulbecco's Essential modified medium (DMEM) with 10\% fetal bovine serum (FBS) and 1\% antibiotics. At 90\% confluence, one dish was treated with 2 $\mathrm{mM}$ MD01145 dissolved in methanol $(\mathrm{MeOH})$ so that the final concentrations of MD1145 and $\mathrm{MeOH}$ were $10 \mathrm{uM}$ and $0.5 \%$ respectively to limit cell toxicity. A negative control dish was treated with an equal volume of $\mathrm{MeOH}$ and the cells were allowed to respond for $12 \mathrm{hrs}$. Cells were harvested using a cell scraper and suspended in $20 \mathrm{~mL}$ of DMEM. The cells were then pelleted by centrifugation at $3000 \mathrm{rpm}$ for 3 mins, washed twice with $5 \mathrm{~mL}$ of phosphate-buffered saline (PBS, $\mathrm{pH} 7.4$ ) to obtain a pellet that was free of FBS protein contaminants.

The cell pellets were subjected to differential detergent extraction (DDE) using the ProteoExtract Subcellular Proteome Extraction kit (Calbiochem) to reduce sample complexity and isolate individual organelle proteomes. According to the supplier's protocol, consecutive incubations of the cells with four extraction buffers produced cytosolic (F1), membraneous $(\mathrm{F} 2)$, nuclear $(\mathrm{F} 3)$ and cytoskeletal $(\mathrm{F} 4)$ cellular fractions [80]. Protein quantitation was attempted using the BioRad protein assay (BioRad) [81] but the assay was incompatible with the detergent concentrations used in the final three extraction buffers. Because DIGE depends on the assumption of equimolar protein concentrations, fraction standardization was still necessary. Hence, assessment of the fraction protein content was performed qualitatively by 1-D SDS PAGE (Figure 5.3). All cell fractions were mixed 1:3 with Laemmli loading buffer and a 12\% resolving gel with a $4 \%$ stacking gel were used for protein separation at $200 \mathrm{~V}$ for 45 mins. One lane was 
loaded with $8 \mu \mathrm{L}$ of pre-stained, broad range protein marker (New England Biolabs). Following an overnight incubation in fixing solution, the protein bands were visualized using Coomassie stain according to the supplier's protocol [82] and silver stained. DIGE was determined to be feasible because a comparison of fractions F1, F2, F3 and F4 between untreated and MD01145-treated lysates showed comparable amounts of protein.

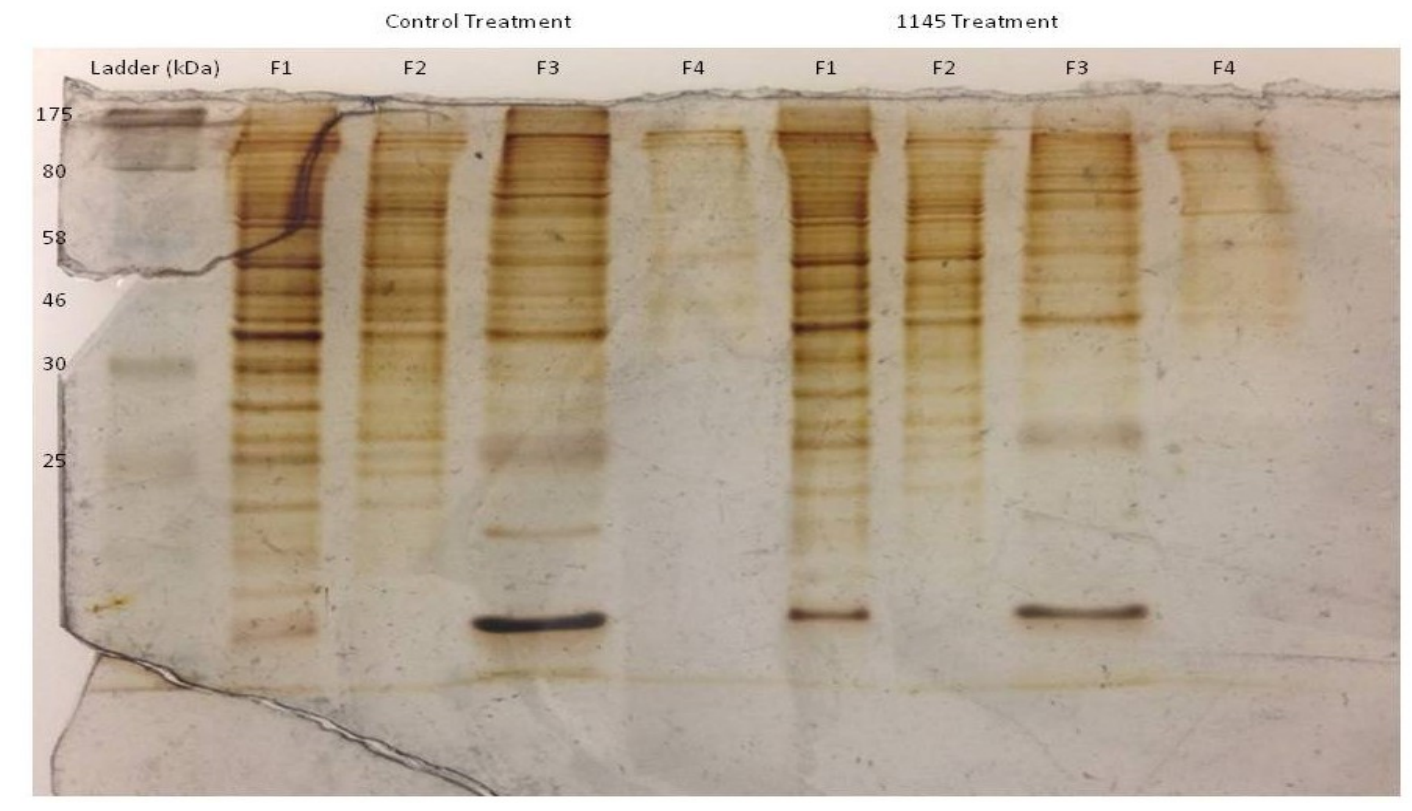

Figure 5.3: Qualitative assessment of protein contents in MD01145-treated and untreated HEK293 fractionated proteomes

The cytosolic and nuclear fractions for both cell treatments were combined and subjected to acetone precipitationto purify the proteins. The fractions were mixed with three volumes of cold acetone for $2 \mathrm{hrs}$. The dried pellets were resuspended in $150 \mu \mathrm{L}$ of resuspension buffer and used to actively hydrate the isoelectric focusing (IEF) strips (7 cm, $\mathrm{pH} 3-10)$ (BioRad) at $50 \mathrm{~V}$ for $12 \mathrm{hrs}$ in the Protean IEF apparatus (BioRad). The proteins were focused at $4000 \mathrm{~V}$ for $2 \mathrm{hrs}$, followed by $10000 \mathrm{~V}$-hr at $4000 \mathrm{~V}$ and held at their $\mathrm{pI}$ at $500 \mathrm{~V}$ according to the manufacturer's recommendations (15). The loaded IPG strips were equilibrated with consecutive DTT and iodoacetamide incubations and loaded 
onto a $12 \%$ SDS resolving gel with an agarose overlay. The SDS-PAGE was run and stained using the same parameters and reference ladder as previously stated (Figure 5.4). The procedure was repeated for combined membraneous and cytoskeletal fractions (Figure 5.5). One upregulated protein spot from the cytosolic/nuclear fraction and one downregulated spot from the membraneous/cytoskeletal fraction were selected for identification. The spots were subjected to in-gel tryptic digestion and the 30 mins proteomics method on the QStar XL mass spectrometer.

Untreated

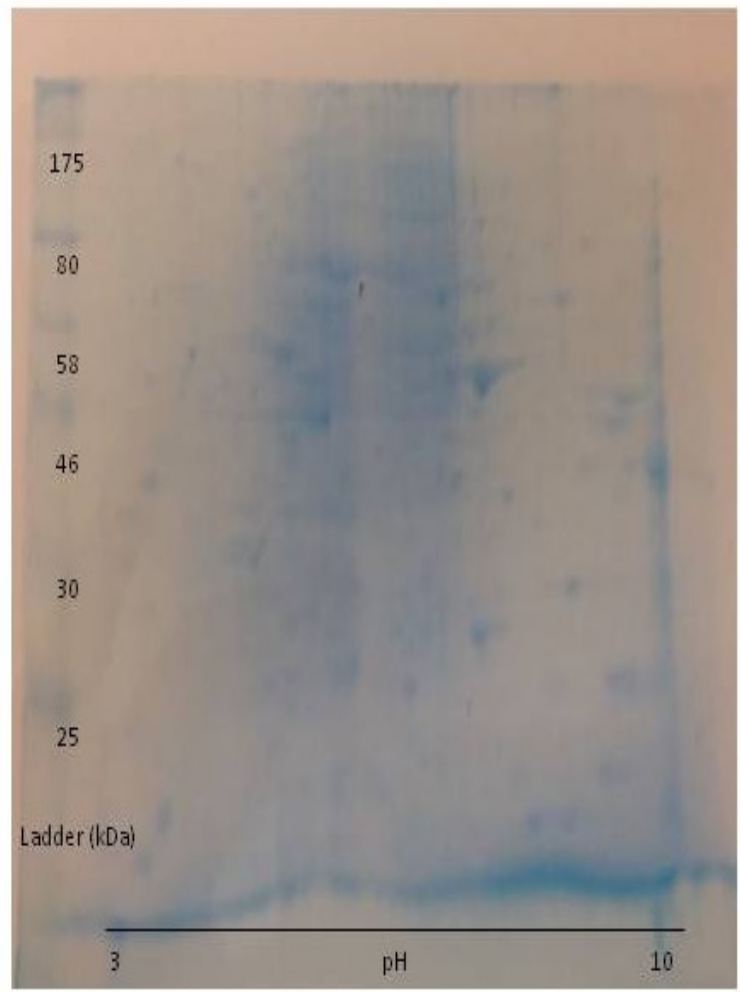

MD01145-treated

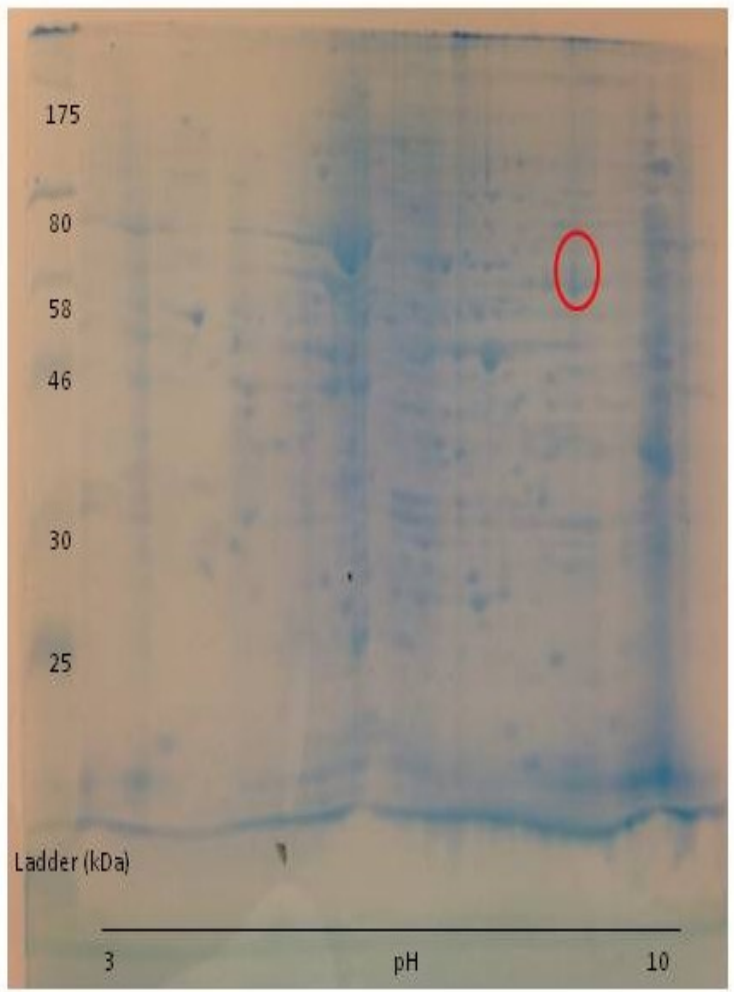

Figure 5.4: DIGE showing changes to the HEK293 F1 and F3 proteomes upon MD01145 treatment 

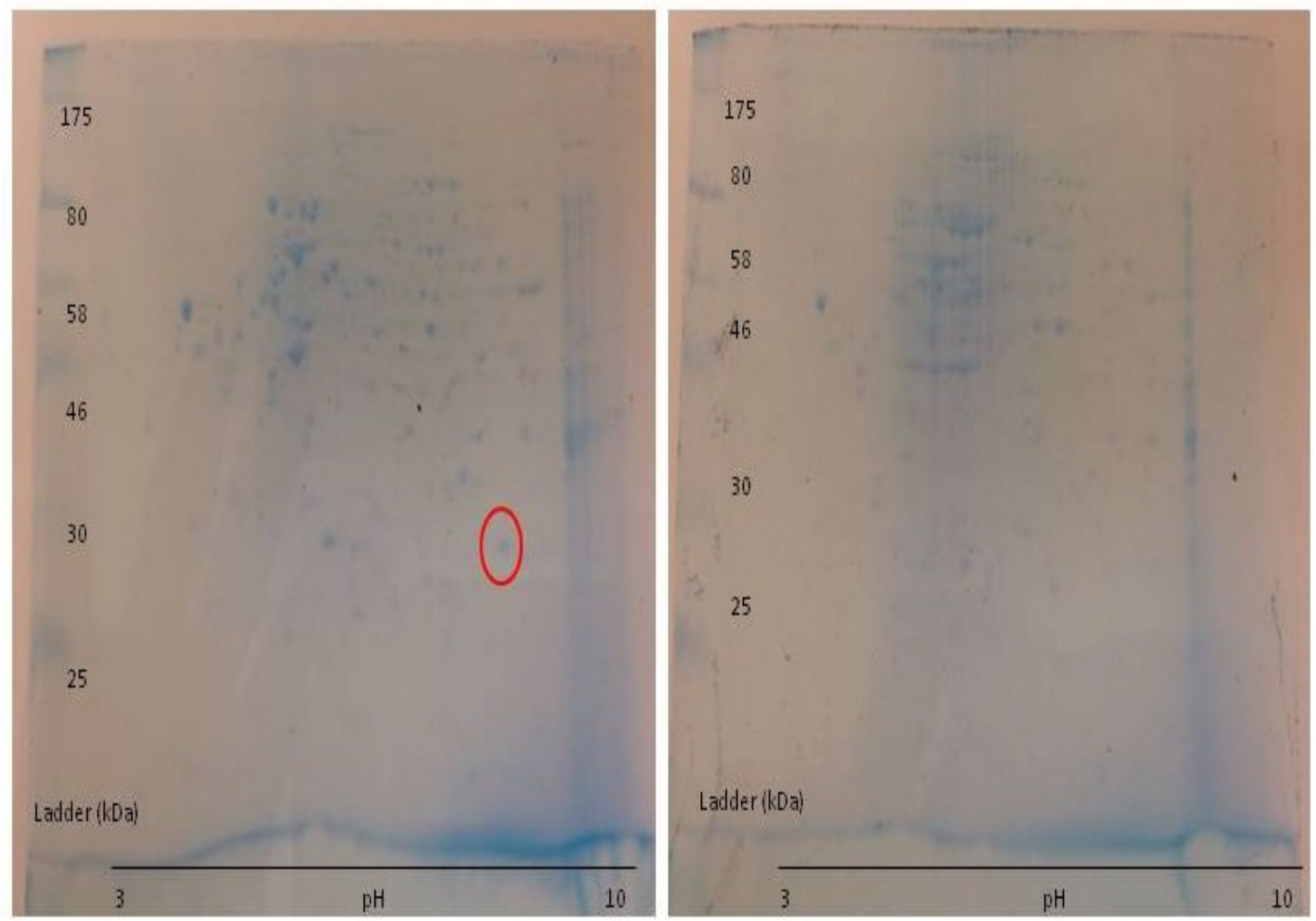

Figure 5.5: DIGE showing changes to the HEK293 F2 and F4 proteomes upon MD01145 treatment

The upregulated protein was identified by MASCOT as pyruvate kinase (PK) (Figure 5.6) with experimentally-sequenced peptides highlighted in red. The MASCOT results strongly support this identification because 10 different peptides were detected accounting for $20 \%$ of the total protein. This produced a high MASCOT score of 351 and the sequenced peptides all showed strong individual scores. Additionally, the predicted molecular weight and pI of PK correspond to the spot location in Figure 5.4.

The enzyme catalyzes the final step of the glycolytic pathway involving the transfer of two phosphate groups from phosphoenolpyruvuate substrate to produce two ATP and two pyruvate molecules. The bioinformatic tool CELLO predicts its most likely 
sub-cellular location to be the cytosol which confirms the efficacy of the DDE process [83]. The detection of a peptide near the N-terminus was important because this is the domain that distinguishes the four PK isozymes. The product ion scan data of this peptide fully supports the isozyme identity because $80 \%$ of y-ion series was detected in the product ion scan and the error associated which each fragment ion was generally within 0.1Da (Figure 5.7). The M2-isozyme was detected in the protein spot, which is expressed more abundantly in some cancer types. PK-M2 over-expression is one reason tumour cells can keep up with their high demands for ATP production [84] and therefore serves as a potential biomarker and cancer drug target [85]. A cancer-specific variant shows less activity which causes an accumulation of PEP that diverts glycolytic intermediate metabolism towards the amino acid biosynthesis pathways that are also in high demand. Consequently, it is hypothesized that if VSes are inhibitors of PK-M2, the cancer cells will not be able to meet the high energy demands of tumour metabolism and be more susceptible to oncolytic viral infection.

Protein sequence coverage: $20 \%$

Matched peptides shown in bold red.

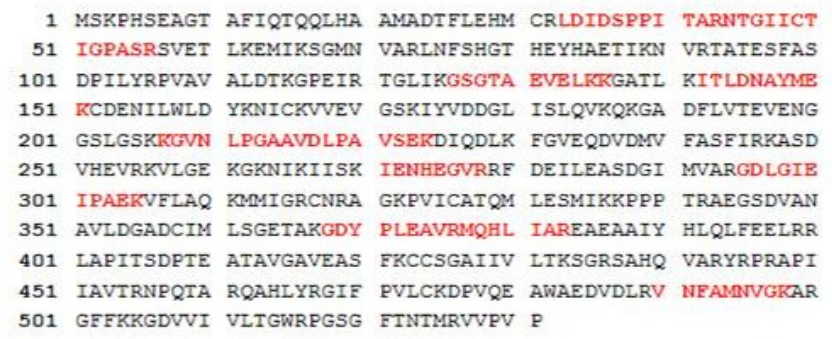

\section{Protein View: gi|35505}

pyruvate kinase [Homo sapiens]

Database: NCBInr

Score: $\quad 351$

Nominal mass $\left(M_{r}\right): 58411$

Calculated pI: $\quad 7.58$

Taxonomy: Homo sapiens

Expect Rank U Peptide

$\begin{array}{llll}0.28 & 1 & \bar{U} & \text { R.LDIDSPPITAR.N }\end{array}$

$12 \frac{1}{1}$ R.NTGIICTIGPASR.S

$41 \quad 1 \quad$ K.GSGTABVELKK.G

$64 \quad \frac{1}{1}$ U $\quad$ K. ITLDNAYMEK.C + Oxidation (M)

$1.4 \mathrm{e}+02 \quad \frac{2}{\mathrm{~N}} \quad$ K.KGVNLPGAAVDLPAVSEK. D

U K. IENHEGVR.R

R. GDLGIEIPAER.V

K. GDYPLBAVR.M

J R. MQHLIAR.E + Oxidation (M)

U R. VNFAMNVGK. A + Oxidation (M)

Figure 5.6: MASCOT results identifying PK as the upregulated $F 1$ protein spot 


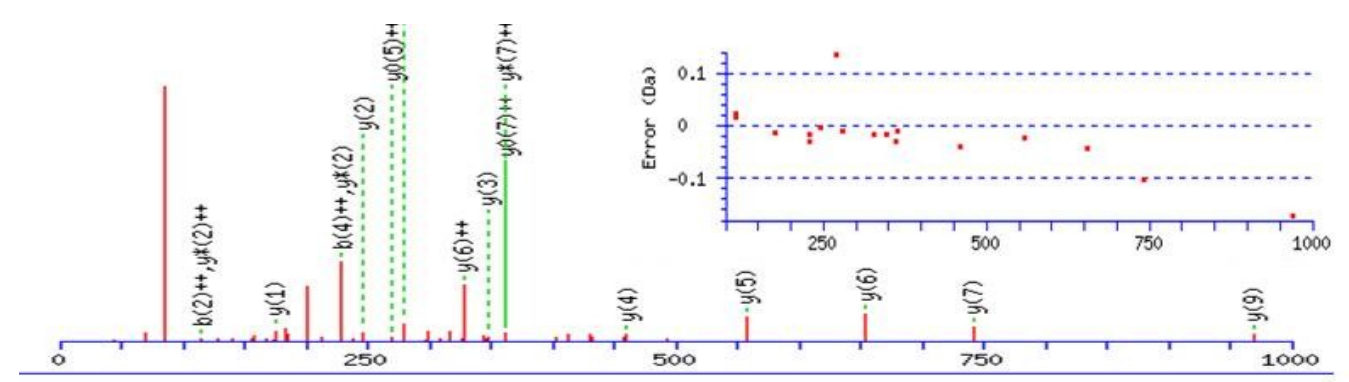

\begin{tabular}{|c|c|c|c|c|c|c|c|c|c|c|c|c|}
\hline$\#$ & b & $\mathbf{b}^{++}$ & $\mathbf{b}^{0}$ & $\mathbf{b}^{0++}$ & Seq. & $\mathbf{y}$ & $y^{++}$ & $\mathbf{y}^{*}$ & $\mathbf{y}^{*++}$ & $y^{0}$ & $\mathbf{y}^{0++}$ & $\#$ \\
\hline 1 & 114.0913 & 57.5493 & & & L & & & & & & & 11 \\
\hline 2 & 229.1183 & 115.0628 & 211.1077 & 106.0575 & D & 1084.5633 & 542.7853 & 1067.5368 & 534.2720 & 1066.5528 & 533.7800 & 10 \\
\hline 3 & 342.2023 & 171.6048 & 324.1918 & 162.5995 & I & 969.5364 & 485.2718 & 952.5098 & 476.7585 & 951.5258 & 476.2665 & 9 \\
\hline 4 & 457.2293 & 229.1183 & 439.2187 & 220.1130 & D & 856.4523 & 428.7298 & 839.4258 & 420.2165 & 838.4417 & 419.7245 & 8 \\
\hline 5 & 544.2613 & 272.6343 & 526.2508 & 263.6290 & $\mathbf{S}$ & 741.4254 & 371.2163 & 724.3988 & 362.7030 & 723.4148 & 362.2110 & 7 \\
\hline 6 & 641.3141 & 321.1607 & 623.3035 & 312.1554 & $\mathbf{P}$ & 654.3933 & 327.7003 & 637.3668 & 319.1870 & 636.3828 & 318.6950 & 6 \\
\hline 7 & 738.3668 & 369.6871 & 720.3563 & 360.6818 & $\mathbf{P}$ & 557.3406 & 279.1739 & 540.3140 & 270.6607 & 539.3300 & 270.1686 & 5 \\
\hline 8 & 851.4509 & 426.2291 & 833.4403 & 417.2238 & I & 460.2878 & 230.6475 & 443.2613 & 222.1343 & 442.2772 & 221.6423 & 4 \\
\hline 9 & 952.4986 & 476.7529 & 934.4880 & 467.7477 & $\mathbf{T}$ & 347.2037 & 174.1055 & 330.1772 & 165.5922 & 329.1932 & 165.1002 & 3 \\
\hline 10 & 1023.5357 & 512.2715 & 1005.5251 & 503.2662 & A & 246.1561 & 123.5817 & 229.1295 & 115.0684 & & & 2 \\
\hline 11 & & & & & $\mathbf{R}$ & 175.1190 & 88.0631 & 158.0924 & 79.5498 & & & 1 \\
\hline
\end{tabular}

Figure 5.7: MASCOT results showing almost complete sequencing of the 33-43 N-terminus PK peptide

The down-regulated protein was identified as annexin (Figure 5.8). Despite only detecting $5 \%$ of the protein sequence and a lower MASCOT score of 68 , this identity is reinforced by the sequencing of two unique peptides with high individual peptide scores. The calcium-dependent phospholipid-binding protein facilitates various cellular activities near the membrane such as exocytosis, endocytosis, cell motility and cell matrix interactions [86]. Its role in membraneous functions further confirms the DDE process efficacy. This result is interesting because past members of our collaboration performed target pull-down experiments and also identified the same annexin isotype.

The 105-115 peptide showed a strong MASCOT score due to accurate fragment ion masses and detection of $60 \%$ of the y-ion series (Figure 5.9) and identified the protein as the A2 isotype. This isotype is strongly linked to pancreatic cancer since annexin A2 knock-down models exhibit limited cell division and proliferation [86]. This information 
contrasts the DIGE experimental findings because the decrease in annexin A2 expression means the protein is more active upon MD01145 treatment. A speculative hypothesis is that VSes enhance the activity of annexin A2 which could facilitate the endocytosis of oncolytic viruses and improve tumour cell infection. This theory is supported by Dziduzscko \& Ozbun who discovered that the protein interacts with human papillomavirus type-16 and increase its uptake into keratinocytes [87]. Nonetheless, the findings from these experiments provided reason to further investigate these proteins as intracellular VSe targets using DIGE.

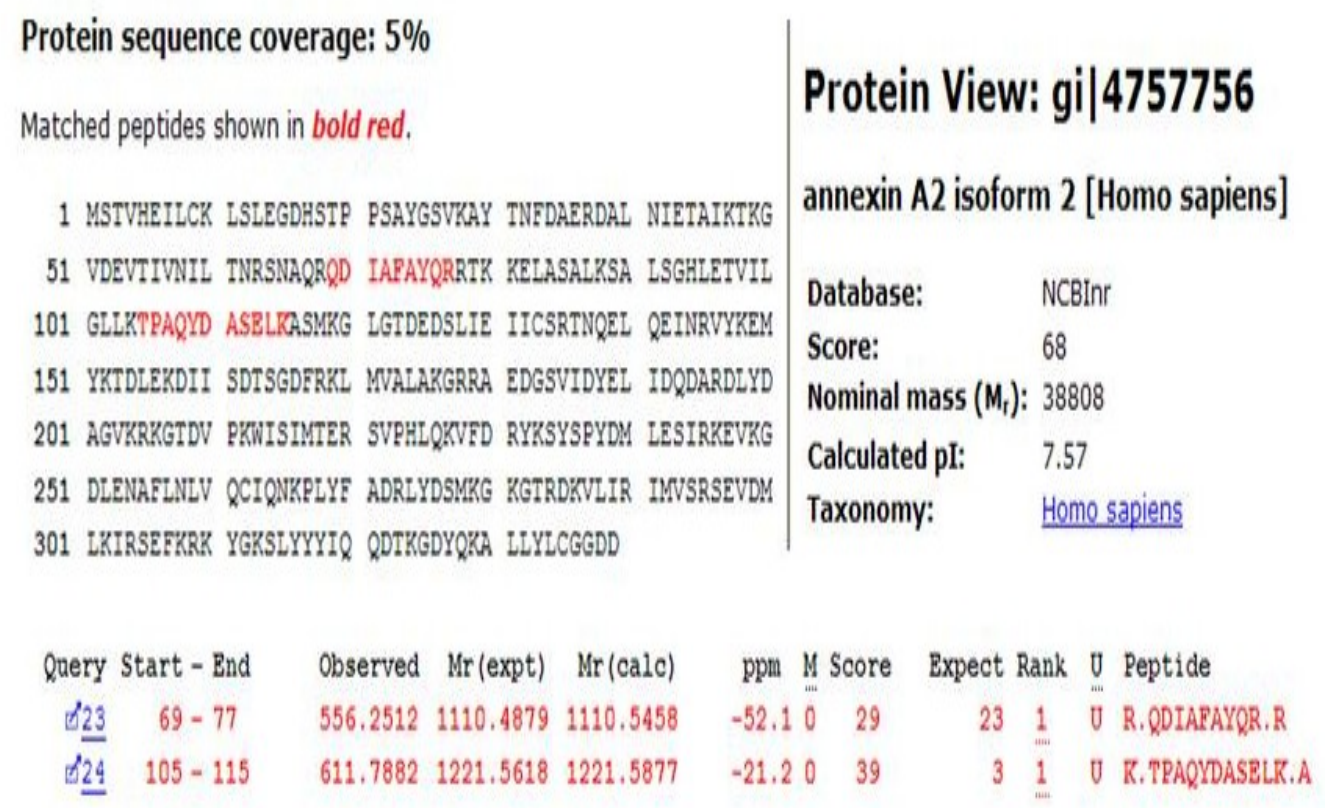

Figure 5.8: MASCOT results showing annexin A2 as the downregulated F3 protein spot 


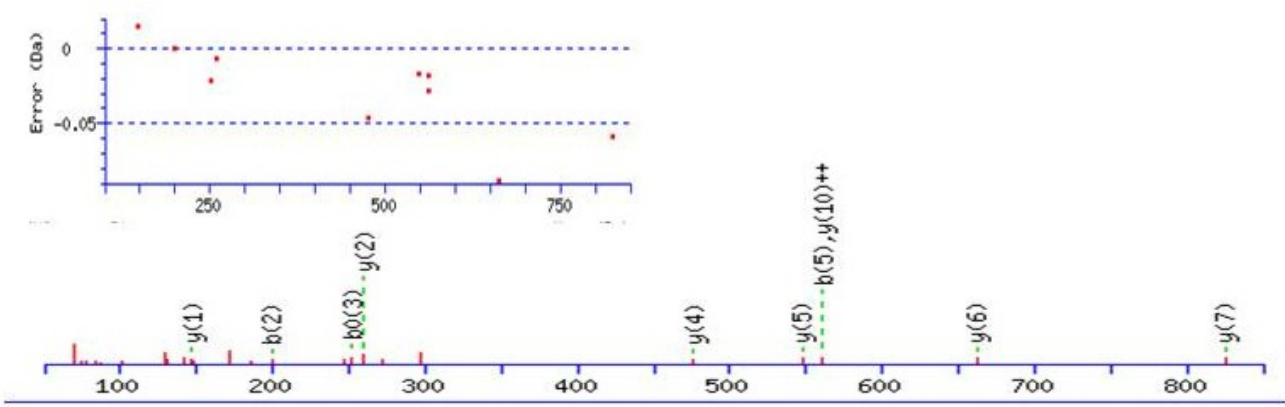

\begin{tabular}{|c|c|c|c|c|c|c|c|c|c|c|c|c|c|c|}
\hline$\#$ & b & $\mathbf{b}^{++}$ & $\mathbf{b}^{*}$ & $\mathbf{b}^{*++}$ & $b^{0}$ & $b^{0++}$ & Seq. & y & $y^{++}$ & $y^{*}$ & $y^{*++}$ & $y^{0}$ & $y^{0++}$ & $\#$ \\
\hline 1 & 102.0550 & 51.5311 & & & 84.0444 & 42.5258 & $\mathrm{~T}$ & & & & & & & 11 \\
\hline 2 & 199.1077 & 100.0575 & & & 181.0972 & 91.0522 & $P$ & 1121.5473 & 561.2773 & 1104.5208 & 552.7640 & 1103.5368 & 552.2720 & 10 \\
\hline 3 & 270.1448 & 135.5761 & & & 252.1343 & 126.5708 & A & 1024.4946 & 512.7509 & 1007.4680 & 504.2376 & 1006.4840 & 503.7456 & 9 \\
\hline 4 & 398.2034 & 199.6053 & 381.1769 & 191.0921 & 380.1928 & 190.6001 & Q & 953.4575 & 477.2324 & 936.4309 & 468.7191 & 935.4469 & 468.2271 & 8 \\
\hline 5 & 561.2667 & 281.1370 & 544.2402 & 272.6237 & 543.2562 & 272.1317 & $\mathrm{Y}$ & 825.3989 & 413.2031 & 808.3723 & 404.6898 & 807.3883 & 404.1978 & 7 \\
\hline 6 & 676.2937 & 338.6505 & 659.2671 & 330.1372 & 658.2831 & 329.6452 & D & 662.3355 & 331.6714 & 645.3090 & 323.1581 & 644.3250 & 322.6661 & 6 \\
\hline 7 & 747.3308 & 374.1690 & 730.3042 & 365.6558 & 729.3202 & 365.1638 & A & 547.3086 & 274.1579 & 530.2821 & 265.6447 & 529.2980 & 265.1527 & 5 \\
\hline 8 & 834.3628 & 417.6851 & 817.3363 & 409.1718 & 816.3523 & 408.6798 & $\mathrm{~S}$ & 476.2715 & 238.6394 & 459.2449 & 230.1261 & 458.2609 & 229.6341 & 4 \\
\hline 9 & 963.4054 & 482.2063 & 946.3789 & 473.6931 & 945.3949 & 473.2011 & E & 389.2395 & 195.1234 & 372.2129 & 186.6101 & 371.2289 & 186.1181 & 3 \\
\hline 10 & 1076.4895 & 538.7484 & 1059.4629 & 530.2351 & 1058.4789 & 529.7431 & $\mathrm{~L}$ & 260.1969 & 130.6021 & 243.1703 & 122.0888 & & & 2 \\
\hline 11 & & & & & & & $\mathrm{~K}$ & 147.1128 & 74.0600 & 130,0863 & 65.5468 & & & 1 \\
\hline
\end{tabular}

Figure 5.9: MASCOT results showing partial sequence coverage of the 105-115 annexin A2 peptide

\subsection{DIGE analysis of the cancerous cell proteome}

To study the VSe MOA more accurately, the HEK293 cells were substituted with 786-0 renal carcinoma cells to represent a tumour model more accurately. The cells were grown to $90 \%$ confluence using the same culture media and treated in a similar manner except the MD01145 was added to the cultures to a higher concentration of $50 \mu \mathrm{M}$. This concentration was below the LD50 and similar to the concentration that elicited the strongest viral sensitization factor. In chapter 4, it became known that MD01145 is not overly stable in aqueous media so the treatment was repeated with the same dose at $3 \mathrm{hr}$ and $6 \mathrm{hr}$ time points following the initial treatment. Cells were harvested in the same manner and counted using a haemocytometer and $0.4 \%$ trypan blue solution (Table 5.1). The cell viability counts ensured the dosage protocol was not toxic because the activation 
of detoxifying pathways could lead to false positive protein expression profiles. This was supported by observing the cells under an EVOS FL microscope (Life Technologies) prior to harvesting which showed no visual signs of cell toxicity (Figure 5.10). Cell counting also permitted the lysate standardization by adding $2 \mathrm{~mL}$ of RIPA lysis buffer (Pierce) to the MD01145-treated cells and $1.64 \mathrm{~mL}$ to the negative control pellet.

Table 5.1: 786-0 cell counts of negative control and MD01145-treated cultures

\begin{tabular}{cccc}
\hline & $\begin{array}{c}\text { Number of Cells } \\
(\mathrm{E}+07)\end{array}$ & Cell Viability $(\%)$ & $\begin{array}{c}\text { Volume of RIPA Buffer } \\
\text { Added }\end{array}$ \\
\hline Treatment & & & $2 \mathrm{~mL}$ \\
$\mathrm{MD} 01145$ & 3.30 & $77( \pm 9)$ & $1.64 \mathrm{~mL}$ \\
\hline
\end{tabular}
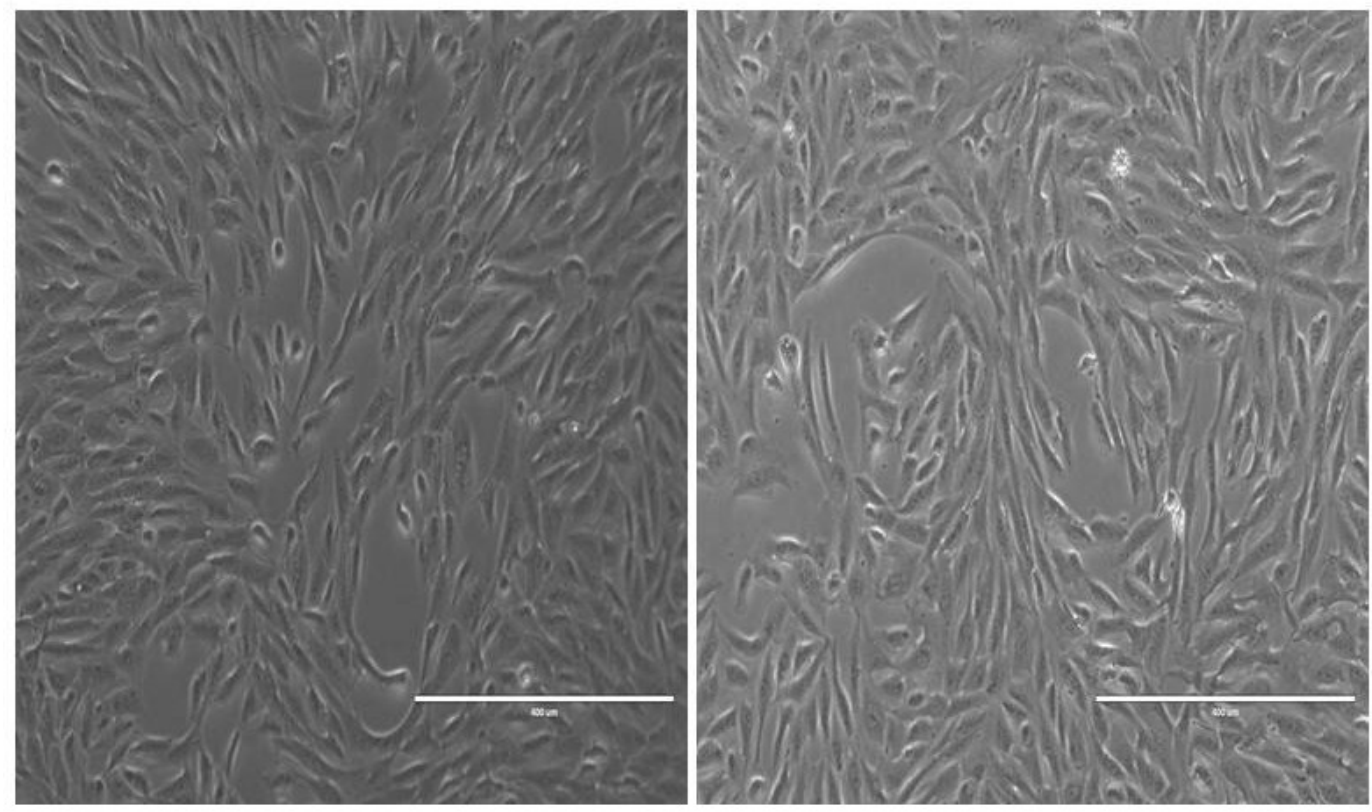

Figure 5.10: Light microscope images (10X) of 786-0 cells treated with $50 \mu \mathrm{M}$ MD01145 (left) and 50 $\mu \mathrm{L} \mathrm{MeOH}$ (right) prior to harvesting 
The bicinchoninic acid (BCA) assay [88] determined the protein concentrations of the MD01145-treated lysate and the control lysate were $8.36 \mu \mathrm{g} / \mathrm{mL}$ and $6.65 \mu \mathrm{g} / \mathrm{mL}$ respectively (Figure 5.11). $3 \mathrm{mg}$ of protein (358 $\mu \mathrm{L}$ of MD01154-treated lysate and 452 $\mu \mathrm{L}$ of control lysate) was dried using the SpeedVac concentrator and resolubilized with $275 \mu \mathrm{L}$ of rehydration buffer. The DIGE protocol was performed as described above. Protein spot resolution was enhanced by separating the proteins on longer IEF strips (17 cm, $\mathrm{pH} 3-10)$ and a larger SDS-PAGE format (20x20 cm instead of mini-gels).

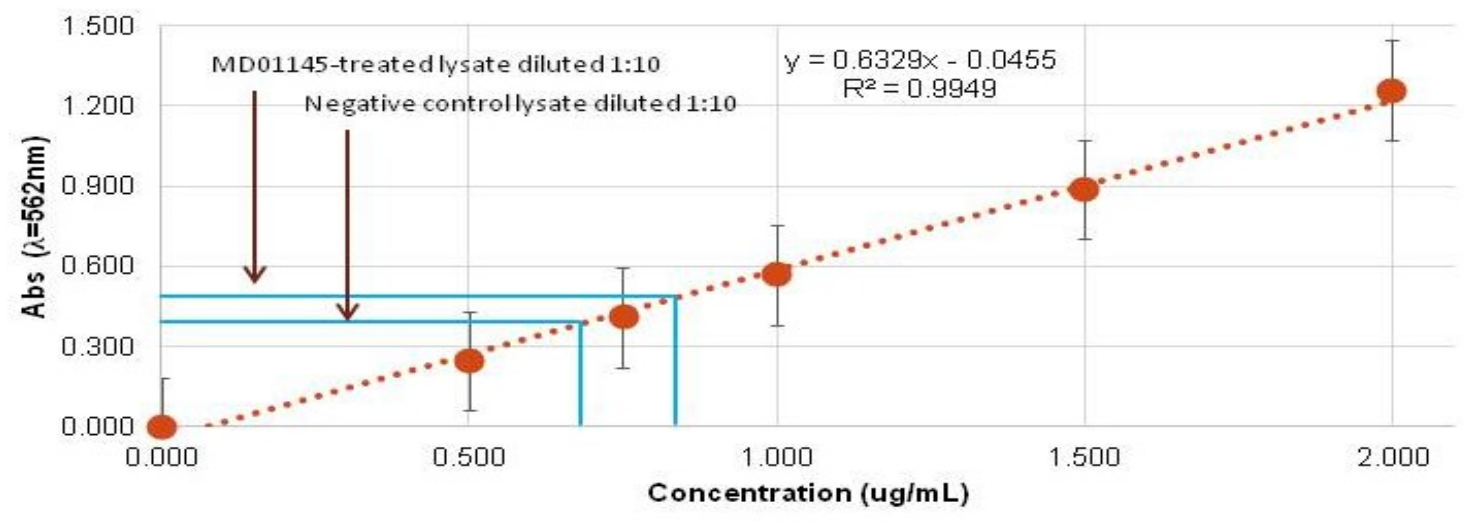

Figure 5.11: Albumin standard curve using the BCA assay. 1:10 diluted cell lysate absorbances are indicated

Few protein spots were observed on the gels with horizontal smearing which was indicative of problems during the rehydration or IEF steps (Figure 5.12). The high salt concentrations in the cell lysate may have negatively affected protein focusing. For the next experiment, the lysates were passed through a spin filter (see Chapter 2) and the proteins were recovered from the inverted filter with the addition of rehydration buffer. A 1D SDS-PAGE was used to qualitatively assess the abundance of proteins in both lysates and ensure that the spin filtration step did not cause a loss of protein (Figure 5.13). Each lane was loaded with $80 \mu \mathrm{g}$ of protein. A comparison between lanes 1 and 3, as well as between lanes 2 and 4 show negligible protein loss as a result of spin filtering. A 
comparison between lanes $1 \& 2$ show that qualitatively, the MD01145-treated and the negative control lysates contain similar protein concentrations as expected from standardization after cell counting.

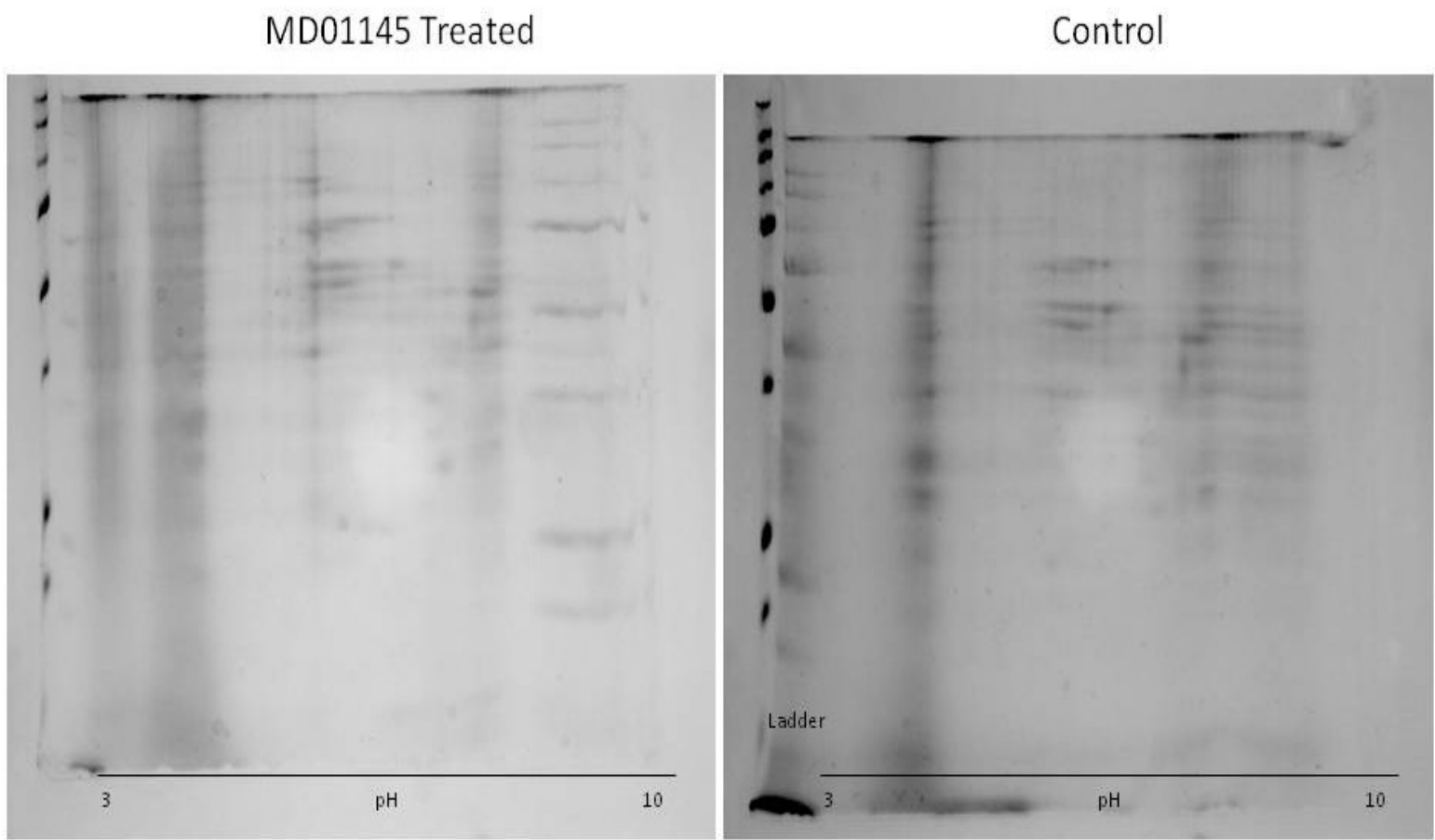

Figure 5.12: DIGE comparing 786-0 proteomes upon VSe treatment. Horizontal streaking is indicative of poor protein IEF.

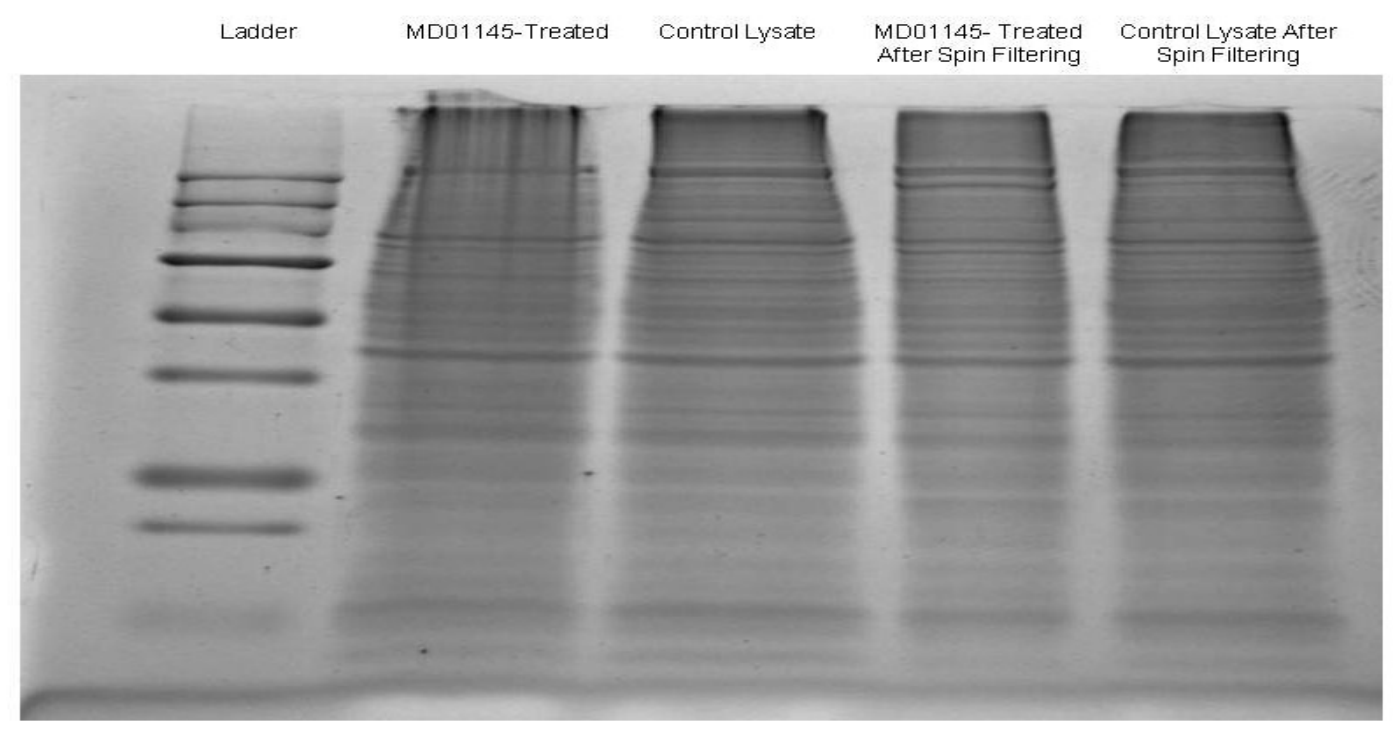

Figure 5.13: Qualitative assessment shows negligible protein loss during spin filtration 
The DIGE experiment was repeated but the horizontal streaking and poor spot resolution were not sufficiently improved (Figure 5.14). The amount of protein loaded onto the IEF strips was the maximum amount recommended for a preparative load. With that said, the disappointing number of protein spots is insufficient to monitor the proteome. This may be attributed to poor protein rehydration and uptake into the IEF strip. If this study were to be repeated, the 786-0 proteins would be precipitated by acetone and trichloroacetic acid to remove lipids and nucleic acids that may interfere with focusing. Ensuring that the Coomassie protein stain is working properly would be worth investigating as well. Another more costly alternative to DIGE is performing a protein micro-array to observe changes to the mRNA expression profile.

MD01145 Treated

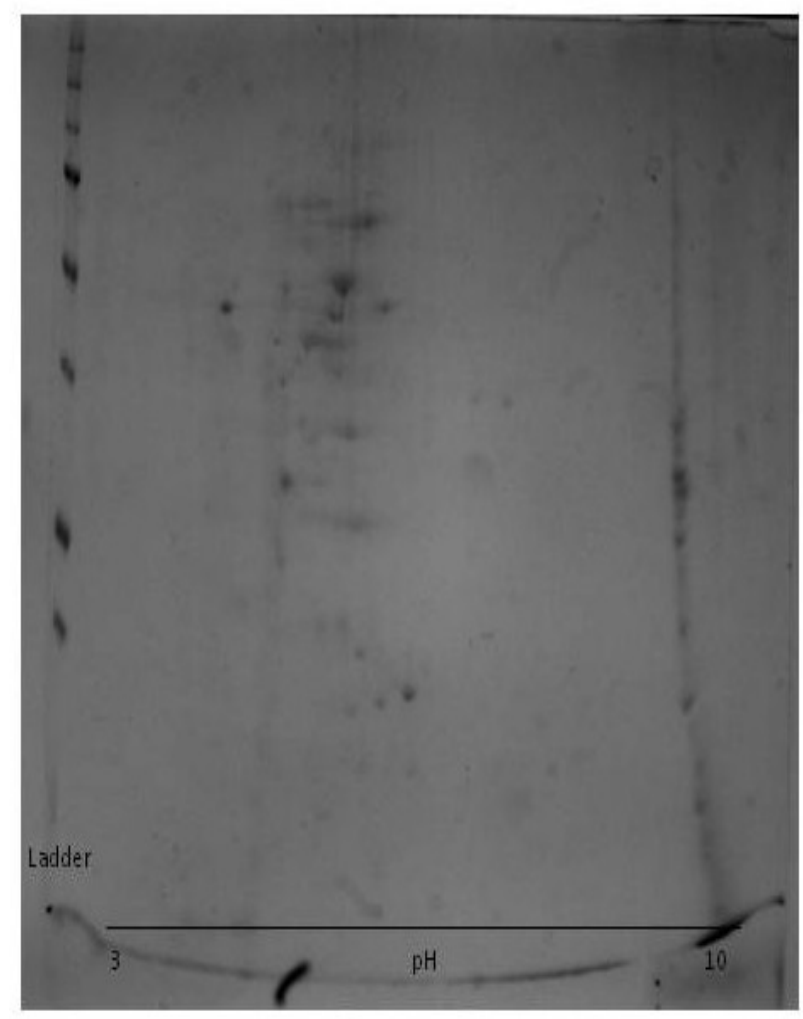

Control

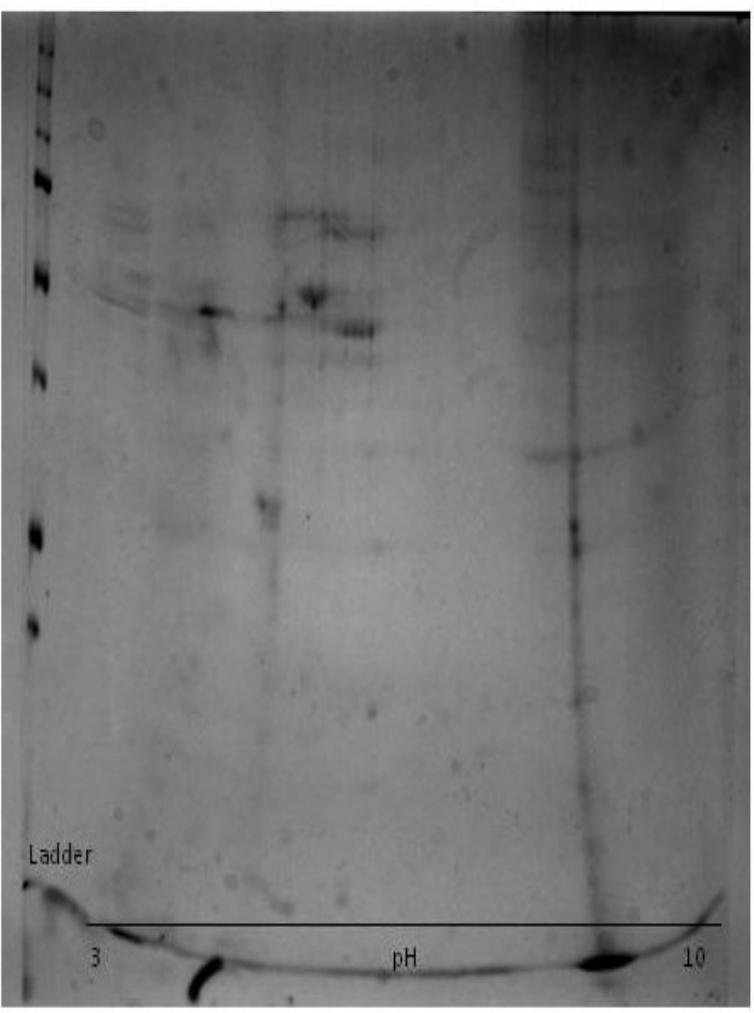

Figure 5.14: DIGE comparing 786-0 proteomes upon VSe treatment. Poor rehydration of the proteins and uptake into the IEF strip may account for the low number of spots 
The culture media was collected before the first, second and third VSe treatments, diluted 1:10 and spin filtered for the purposes of absolute MD01145 quantitation by MRM analysis on the 4000 Qtrap MS (Figure 5.15). The concentration of the stock was expected to be $5 \mu \mathrm{M}$ (column 2) but was found to be $3.6 \mu \mathrm{M}$ (column 1). In hindsight of the VSe QC experiments in Chapter 3, preparation of the VSe stock in DMSO was not optimal. From Chapter 4, it was found that MD01145 is $19.1 \%$ stable in blood plasma. Assuming this would translate in cell culture media, it was expected that the MD01145 concentration after $3 \mathrm{hrs}$ of culture media incubation would degrade to $0.99 \mu \mathrm{M}$ (column 3). No MD01145 was detected in the culture media before treatment as expected (column 4). The culture media contained $0.21 \mu \mathrm{M}$ MD01145 $3 \mathrm{hrs}$ after the first treatment which yields a $4.3 \%$ stability. This significant loss in stability could be a result of cellular VSe uptake. The culture media contained 1.01 $\mu \mathrm{M}$ MD01145 $3 \mathrm{hrs}$ after the second treatment. This is an indication that the VSe is conjugating to its intracellular target that is becoming increasingly saturated with each new treatment, thus leaving more MD01145 in the cell media.

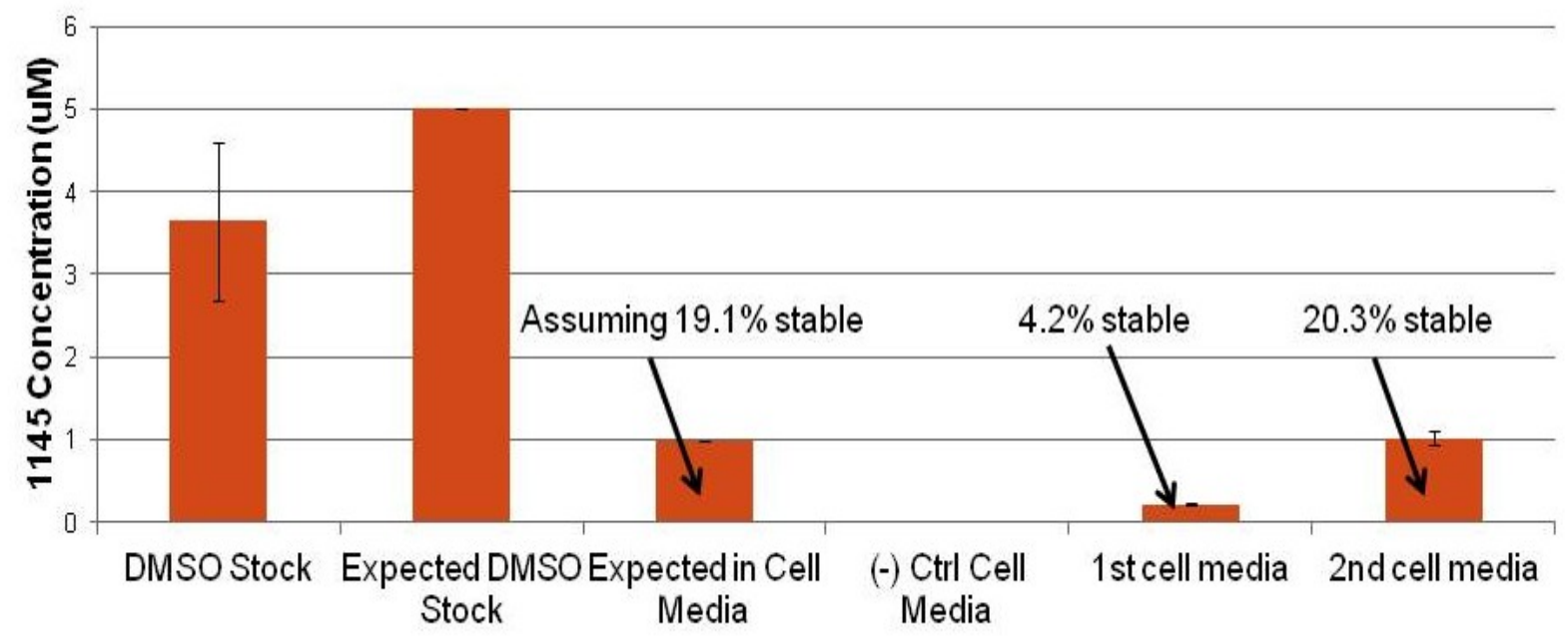

Figure 5.15: MD01145 quantitation in cell culture media shows potential saturation of the VSe target 


\subsection{Affinity pull-down of VSe targets}

A more specific method of small molecule target identification that falls under ABPP chemical proteomic studies is affinity chromatography using probes. The active probe is based from the structure of MD01145 with the replacement of the phenyl group with a carboxylic acid with a triazole linker. The inactive probe was not expected to elicit a viral sensitization effect because the essential $\beta-\mathrm{Cl}$ was substituted with an isobutylamine group (Figure 5.16).

Active Probe

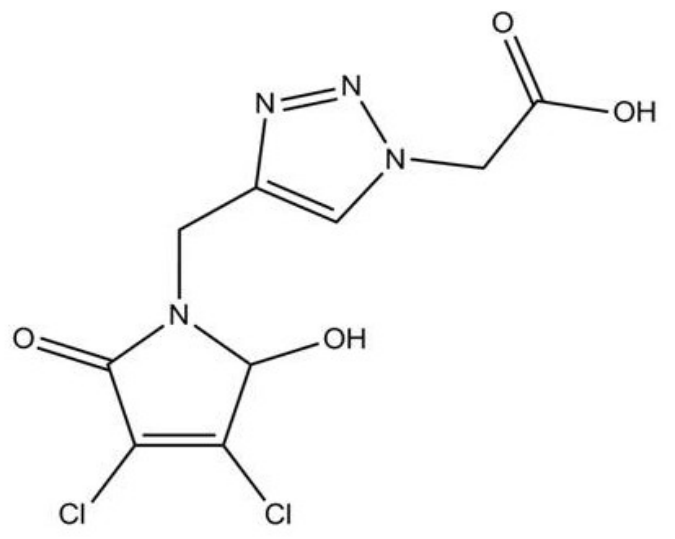

Inactive Probe

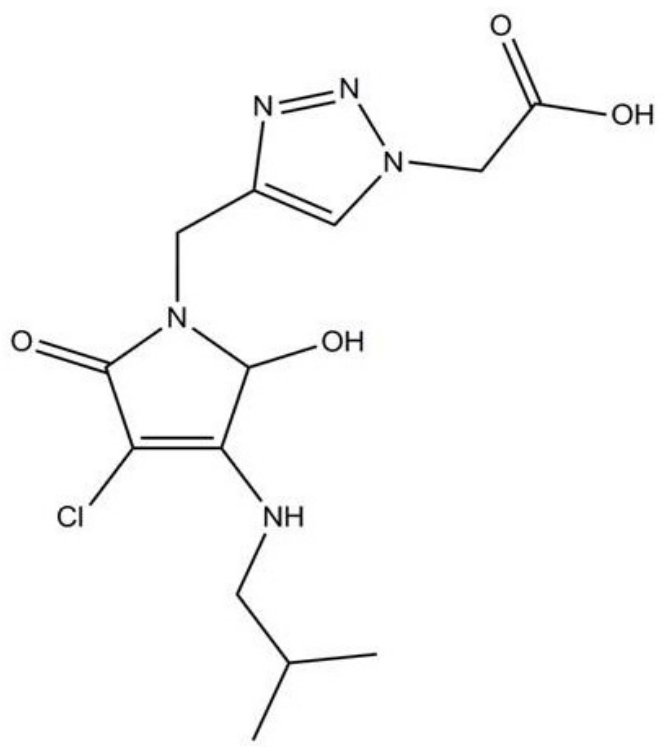

Figure 5.16: The active and inactive MD01145 probes used for sepharose bead conjugation

The probes were coupled to sepharose beads by activating the carboxylic acid group using N-N-diisopropyl-carbodiimide (DIPCDI) and substituting it with 1hydroxybenzotriazole (HOBT). This created a strong leaving group to be replaced by the free amide linked by an 11-C spacer arm to sepharose beads (GE Healthcare) in a 3:1 probe-to-bead ratio (Figure 5.17) [89]. The coupling efficiency was assessed using the 
trinitrobenzene sulfonic acid (TNBS) test for free amines. A small amount of coupled beads were transferred using the tip a Pasteur pipette to a tube containing 2 drops of diisopropylethylamine (DIPEA) in dimethylformamide (DMF) and 2 drops of $1 \mathrm{M}$ picrylsulfonic acid. Uncoupled amines react to produce a red-orange colour after 10 mins at room temperature [89]. The active and inactive bead solutions produced a yellow colour while the uncoupled beads produced a deep red colour as evidence that the coupling reaction was successful.

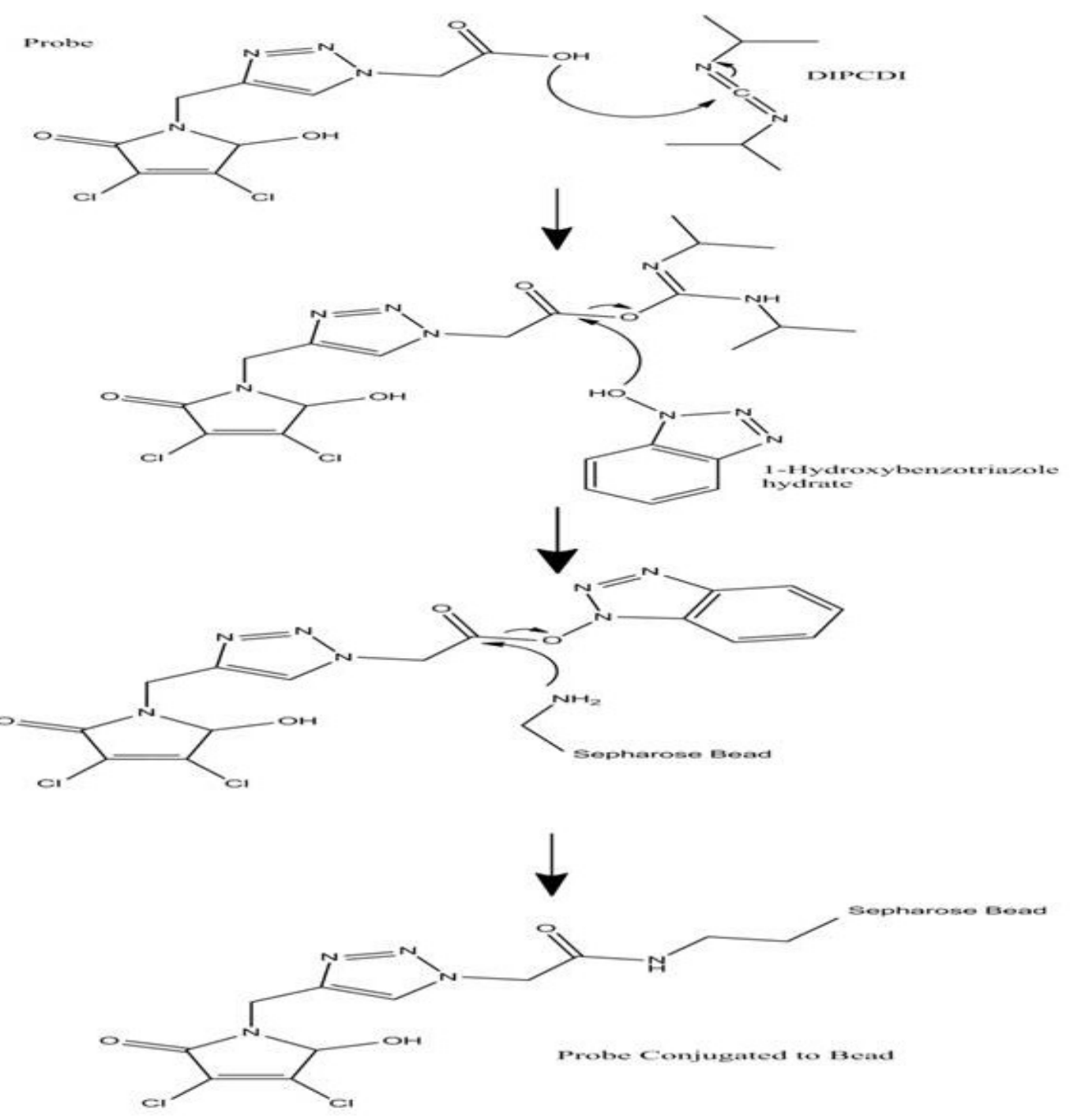

Figure 5.17: The coupling of MD01145-based probes to free amine sepharose beads through carboxylic acid activation 
Prior to harvesting, 786-0 cells were infected with VSV $\Delta 51$ or left uninfected in the chance of observing virus-specific targets. The cells were lysed using a $0.5 \%$ Tritonbased lysis buffer and a Bradford assay quantified the protein concentration to be 0.86 $\mathrm{mg} / \mathrm{mL}$. For each sample, $200 \mu \mathrm{L}$ of bead slurry was washed three times with equal volumes of lysis buffer. The active probe, inactive probe and uncoupled sepharose beads were incubated with $260 \mu \mathrm{L}$ of lysate for $12 \mathrm{hrs}$ or $1 \mathrm{hr}$ at $4^{\circ} \mathrm{C}$. Non-specific proteins were washed away three times with $500 \mu \mathrm{L}$ of lysis buffer and the finals washes were collected for SDS-PAGE. Specifically-bound proteins were eluted by boiling the beads in $12 \mu \mathrm{L} 2 \mathrm{X}$ Laemmlli buffer for 10 mins and analyzed by SDS-PAGE. Protein bands were tryptically digested and identified using the QStar XL mass spectrometer.

Bands representing myosin and beta-actin were observed for samples using the active probe as well as the inactive probe and unconjugated beads, thus classifying their interactions as non-specific (Figure 5.18). When the probes were incubated for $1 \mathrm{hr}$ instead of twelve, non-specific protein staining was much fainter indicating that longer incubation times were necessary to pull-down the VSe target (Figure 5.19). No protein bands were observed in the final washes of the beads indicating that three washes were sufficient to remove loosely interacting non-specific proteins (Figure 5.20). On the same gel, virally infected and uninfected lysates were used as a positive control to confirm the presence of various proteins. 


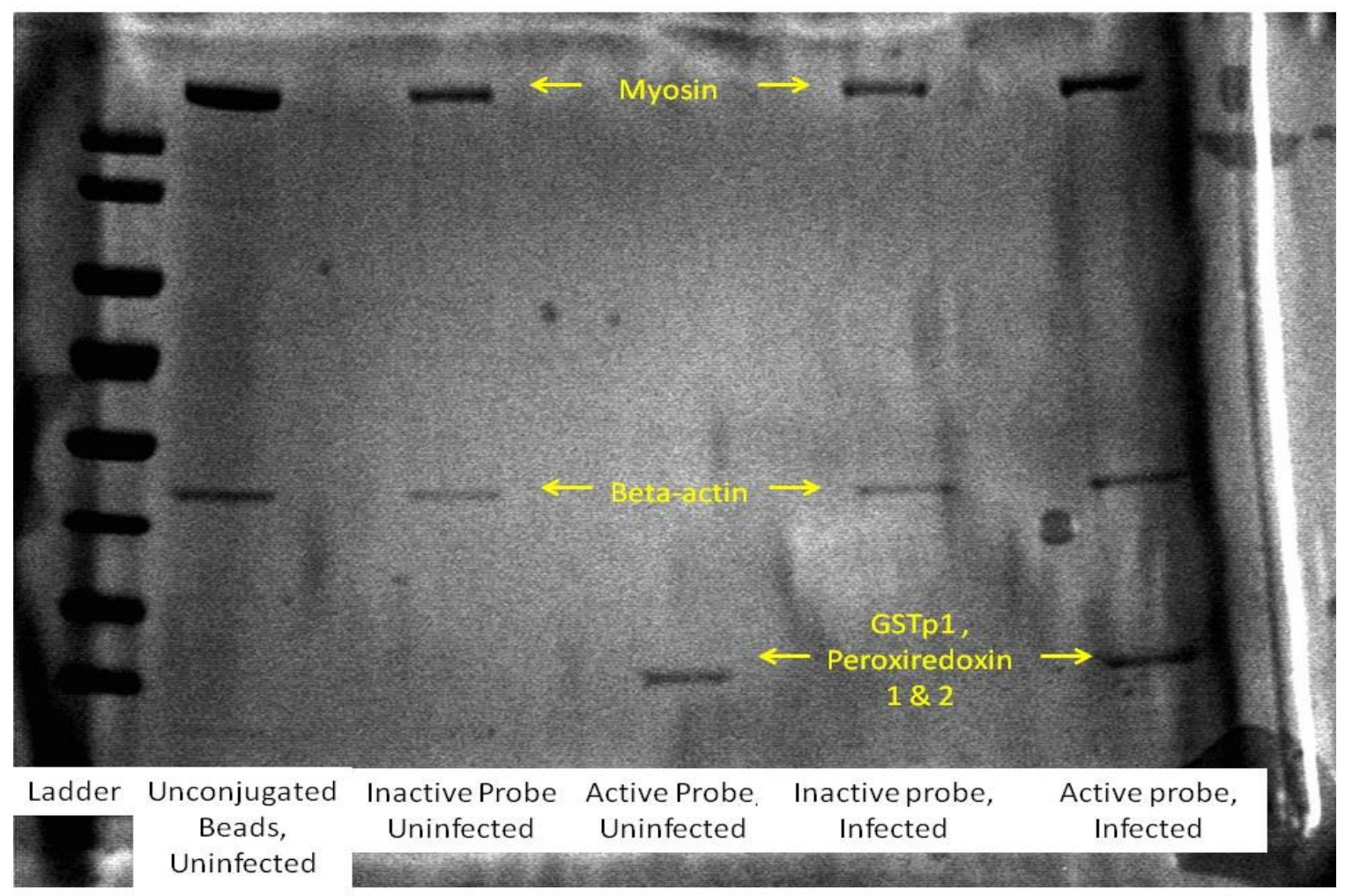

Figure 5.18: VSe targets pulled down using active, inactive and unconjugated beads after a $12 \mathrm{hr}$ incubation with lysate uninfected and VSVD51 incubation

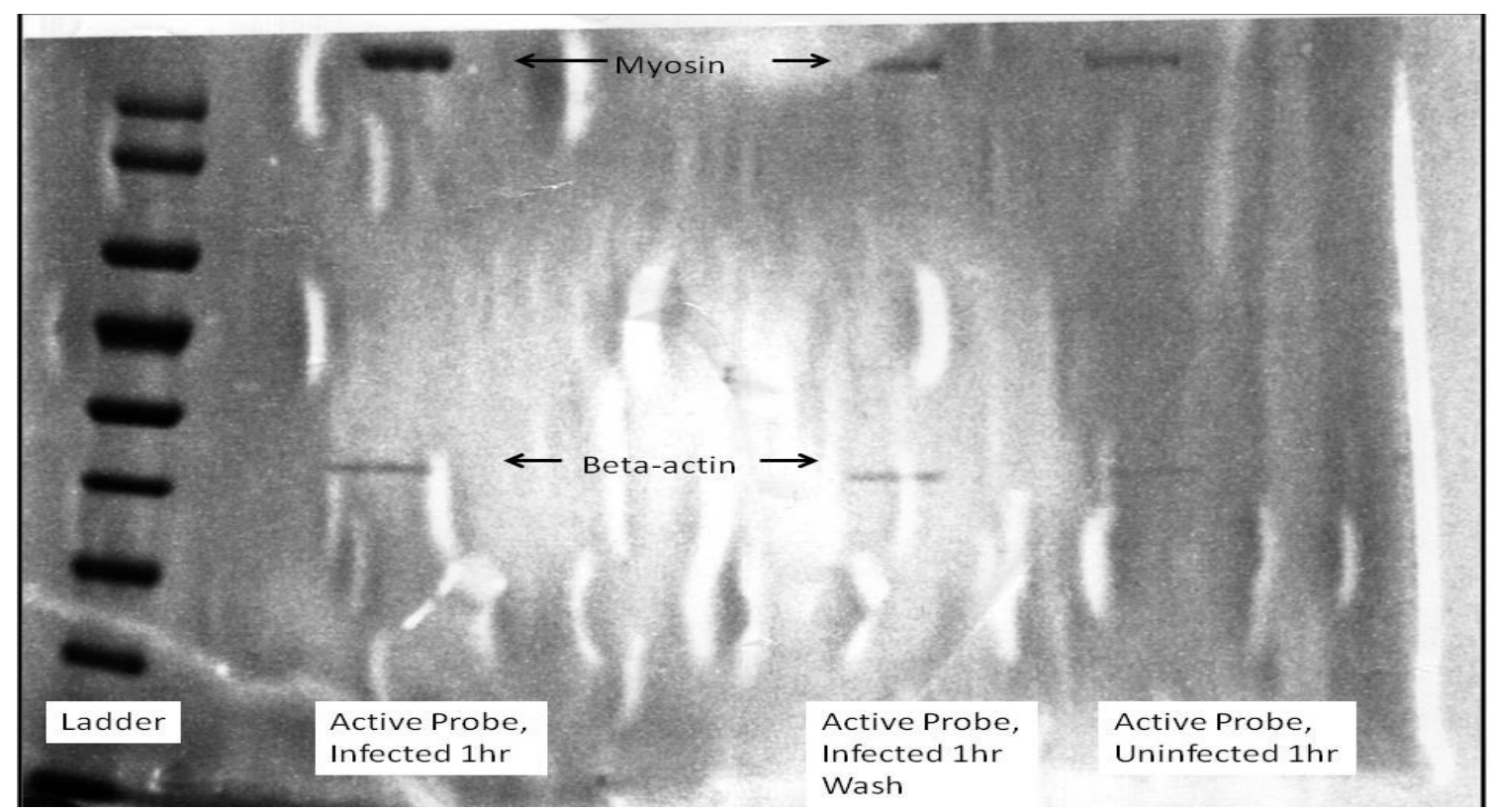

Figure 5.19: VSe targets pulled down using an active probe after $1 \mathrm{hr}$ incubation with uninfected and VSV $\Delta 51$ infected lysates 


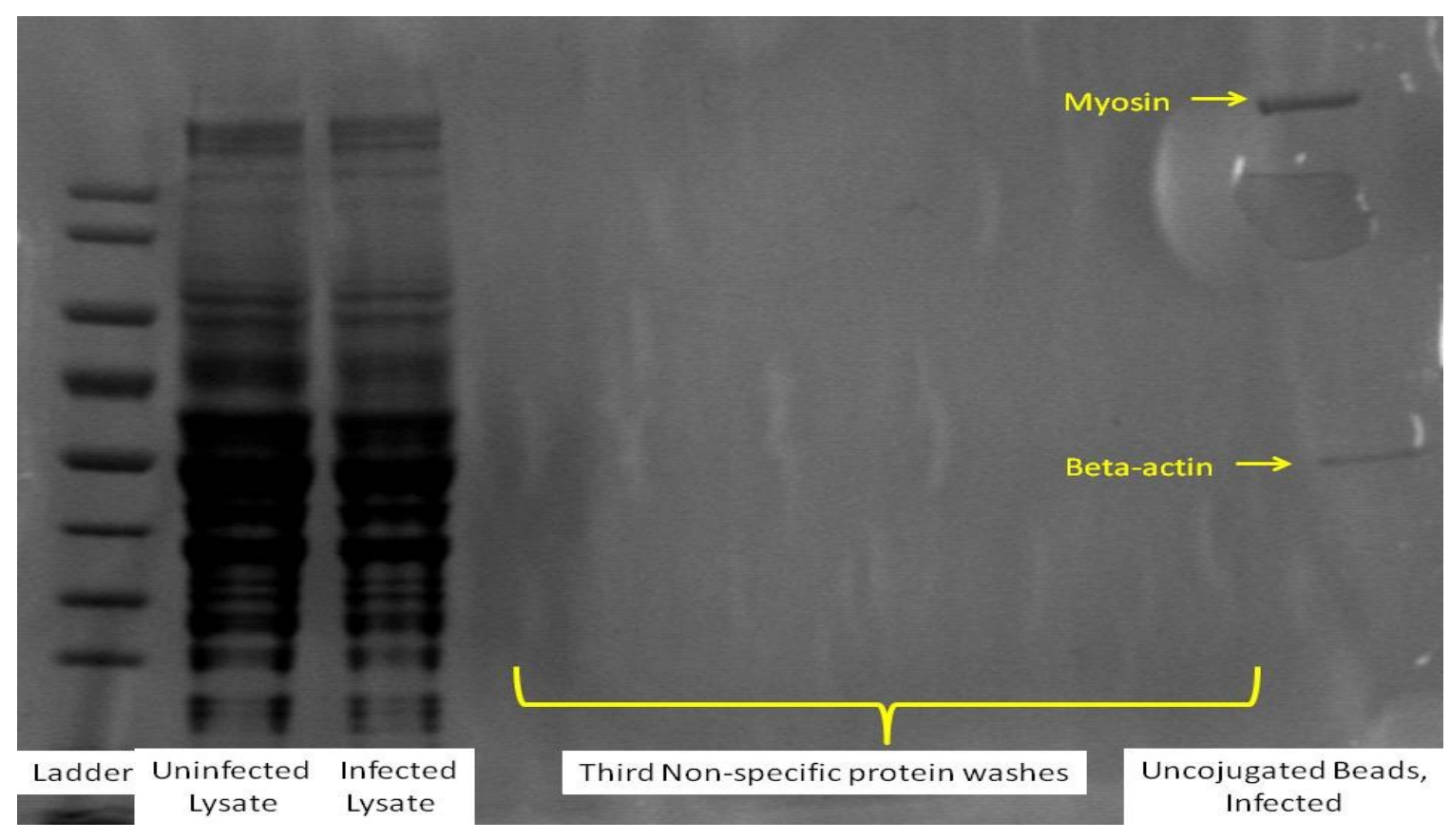

Figure 5.20: Final washes contained no observable proteins while the lysates proved the presence of numerous proteins available for VSe targeting

One protein band was specific to the positive probe samples in Figure 5.18 which contained glutathione s-transferase p1c (GSTp1), peroxiredoxin-1 (PR1) and peroxiredoxin-2 (PR2). This was considered odd because the chances of simultaneously pulling down three proteins with the same molecular weight were slim but all three proteins have similar molecular weights at $23 \mathrm{kDa}$.

Two unique GSTp1 peptides were detected to represent $10 \%$ of the protein sequence (Figure 5.21) with one peptide showing a high MASCOT score of 64 that fragmented to produce $90 \% \mathrm{y}$-ion series (Figure 5.22). The two peptides that were sequenced permitted distinction of the P-class from the other classes of GST. GSTp1 is a member of the family of enzymes responsible for the deprotonation of glutathione and its conjugation to electrophiles for the purposes of detoxification. The P-class is strongly linked to the progression of several cancers including ovarian, breast and colon 
carcinomas because it is suggested that overexpression of GSTp1 leads to chemotherapy resistance [90]. If probe does target GSTp1, this creates a link between VSes and making cancer cells more susceptible to OVt. The identification of GSTp1 as a potential VSe target prompted the glutathione stability tests mentioned in Chapter 4 and enzyme inhibition assay described in the following section.

\section{Protein sequence coverage: $10 \%$}

Matched peptides shown in bold red.

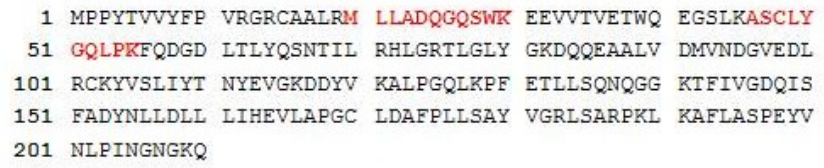

1 MPPYTVVYFP VRGRCAALRM LLADQGQSWK EEVVTVETWQ EGSLKASCLY

51 GQLPKKQDGD LILYQSNTIL RHLGRTLGLY GKDQQEAALV DMVNDGVEDL 101 RCKYVSLIYT NYEVGKDDYV KALPGQLKPF ETLLSQNQGG KTFIVGDQIS 151 FADYNLLDLL LIHEVLAPGC LDAFPLLSAY VGRLSARPKL KAFLASPEYV 201 NLPINGNGKQ

$\begin{array}{rcrrr}\text { Query } & \text { Start - End } & \text { Observed } & \text { Mr (expt) } & \text { Mr (calc) } \\ \varangle 42 & 20-30 & 638.8779 & 1275.7412 & 1275.6281 \\ \square 45 & 20-30 & 646.8748 & 1291.7350 & 1291.6231 \\ \square 35 & 46-55 & 568.8439 & 1135.6732 & 1135.5696\end{array}$

\section{Protein View: gi|726098}

glutathione S-transferase-P1c [Homo sapiens]

Database: $\quad$ NCBInr

Score: $\quad 100$

Nominal mass $\left(M_{r}\right): 23583$

Calculated pI: $\quad 5.43$

Taxonomy: Homo sapiens

Figure 5.21: MASCOT results for GSTp1 represented by two class-specific peptides

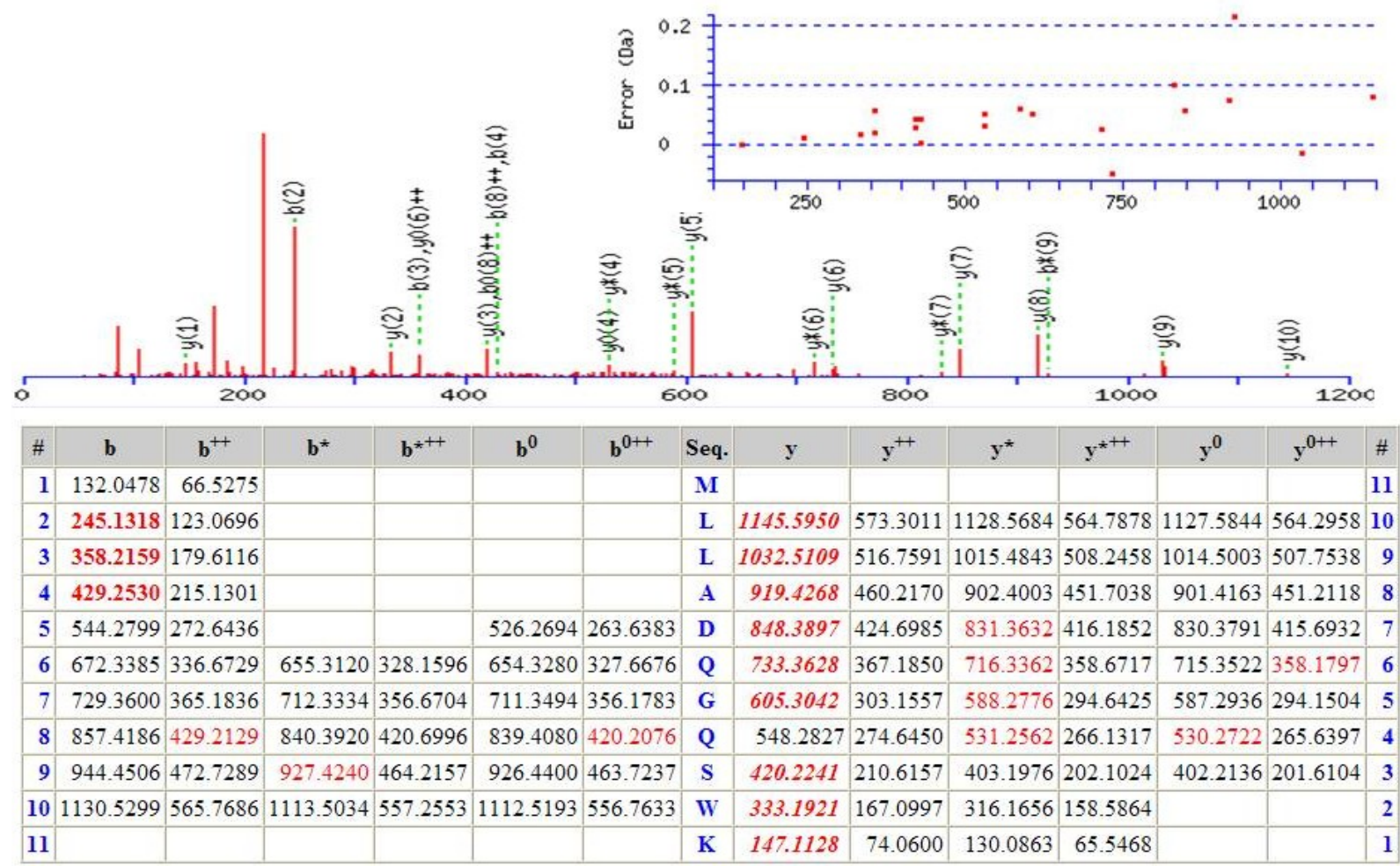

Figure 5.22: Fragmentation of the first GSTp1 peptide to produce $90 \%$ of the $y$-ion series with consistent mass accuracy 
PR1 and PR2 were represented by 3 and 2 peptides to produce MASCOT scores of 155 and 116 (respectively) (Figures 5.23 and 5.24). The anti-oxidant enzymes belong to the family of enzymes responsible for the reduction of $\mathrm{H}_{2} \mathrm{O}_{2}$. Similarly to GSTp1, PR is associated with detoxification. Additionally, it can use reduced glutathione as a substrate and the enzyme is often linked to GST; the P-class more specifically. Overexpression of PR is linked to breast cancer [91], thus targeting this enzyme will increase a tumour's susceptibility to the stress induced by OVt. Alternatively, pulling down detoxifying enzymes using electrophilic probes should not be surprising and they could simply be unintended off-targets of VSes.

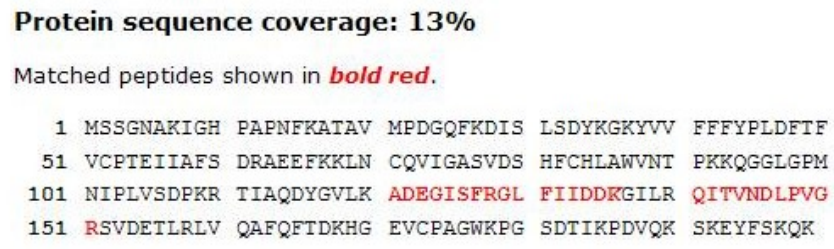

Figure 5.23: MASCOT results for PR1 represented by three unique peptides

\begin{tabular}{|c|c|c|c|c|}
\hline Score: & & 15 & & \\
\hline Nomina & mass (M & $\left.a_{r}\right): 22$ & 324 & \\
\hline Calcula & ed pI: & & 27 & \\
\hline Taxono & & & mo & sapiens \\
\hline Score & Expect & Rank & $\underline{\sigma}$ & Peptide \\
\hline 41 & 2.2 & 1 & $\overrightarrow{0}$ & K.ADEGISFR.G \\
\hline 47 & 0.29 & 1 & & R.GLFI IDDK.G \\
\hline 68 & 0.0014 & 1 & & R. QITVINDLPVGR. \\
\hline
\end{tabular}

Protein sequence coverage: $10 \%$

Matched peptides shown in bold red.

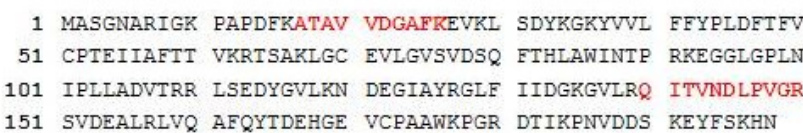

Protein View: gi|438069

thiol-specific antioxidant protein [Homo sapiens]

\begin{tabular}{ll} 
Database: & NCBInr \\
Score: & 116 \\
Nominal mass $\left(\mathrm{M}_{\mathrm{r}}\right):$ & 22014 \\
Calculated pI: & 6.84 \\
Taxonomy: & Homo sapiens \\
\hline
\end{tabular}

Taxonomy: $\quad$ Homo sapiens

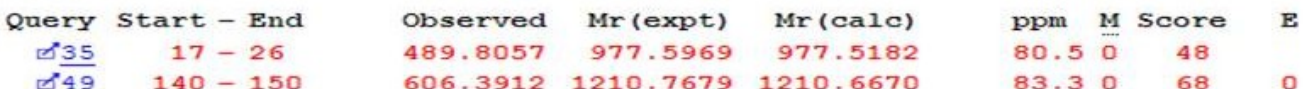

Figure 5.24: MASCOT results for PR2 represented by two unique peptides 


\subsection{GSTp1 inhibition assay}

GSTp1 was cloned and purified in Escherichia coli by colleagues at the University of Guelph. $50 \mu \mathrm{g}(2 \mu \mathrm{M})$ of the enzyme was incubated in PBS with either 1 $\mathrm{mM}$ GSH, $25 \mu \mathrm{M}$ VSe1 or both compounds. The small molecules and enzyme were separated by spin filtration so that the enzyme could be tryptically digested in solution. VSe1, GSH and their adduct were quantified using the "30 min sensitizer" full mass scan method on the QStar XL instrument. This revealed that VSe1 is readily reacting with glutathione, even in the absence of enzyme, since in both instances they are in solution together the VSe1 signal disappears and the adduct signal appears. In the presence of enzyme, neither VSe nor glutathione-conjugate significantly decreased in ion intensity, thus indicating that covalent inhibition is unlikely (Figure 5.25). Additional experimental replicates of this study would instill further confidence in the results. This approach of proving GSTp1 modification by VSe1 is not optimal because the concentrations of the small molecules are in great excess of the enzyme. Therefore even if covalent inhibition was occurring, only a negligible decrease in small molecule ion signal would be observed. A proteomics-based strategy was more accurate. 


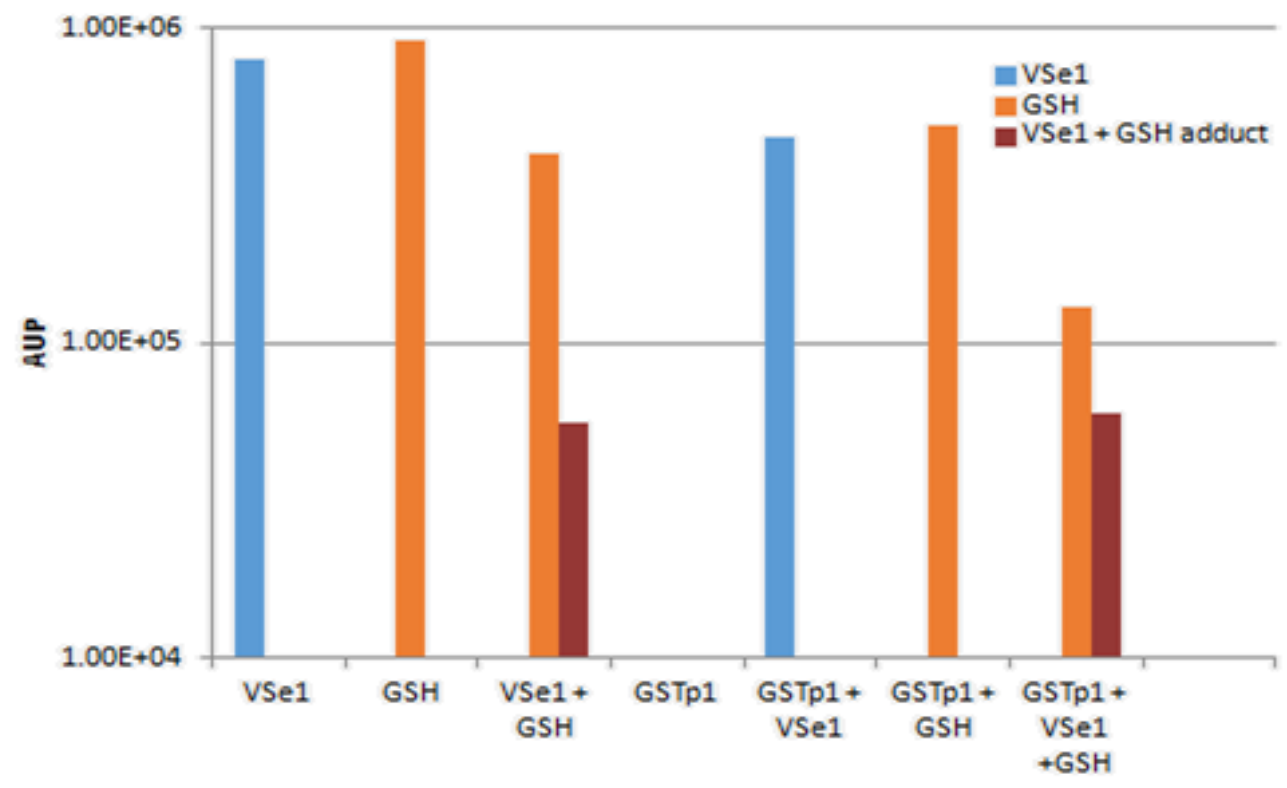

Figure 5.25: GSTp1-VSe1 reaction kinetics analyzed by LC-MS shows no signs of covalent inhibition

$46 \%$ peptide sequence coverage was achieved for the digested GSTp1 proteins (Figure 5.26). A few approaches were taken to observe VSe1-conjugated peptides. Similar to the conjugation of GSH with MD01145 in Chapter 4, it was predicted that VSe1-modified peptides would be formed by nucleophilic addition at the $\beta$-carbon. Thus, $\left[\mathrm{M}^{37} \mathrm{Cl}+2 \mathrm{H}\right]^{+2}$ peptide ions with $33 \%$ intensity relative to the $\left[\mathrm{M}^{35} \mathrm{Cl}+2 \mathrm{H}\right]^{+2}$ ion would be observable in the full mass scan of the enzyme digests that were incubated with VSe1. This was not the case as multiply charged ions with ${ }^{37} \mathrm{Cl}$ labels were not detected. If VSes were to be covalent inhibitors of GSTp1, it would be expected that the highly conserved active site Cys47 would be a likely site of conjugation [92]. The tryptic peptide that includes Cys47 was sequenced for all of the enzyme digests, including those incubated with VSe1, which signifies that this amino acid remains unmodified. Cysteine is a likely site of conjugation due to side chain's nucleophilic thiol group. Only one of four cysteine residues were sequenced in the VSe1-present samples, thus three other cysteines are still 
capable of providing a site for conjugation. For more conclusive evidence that VSe1 does not covalently react with a cysteinyl thiol, the sequence coverage would have to be significantly increased. This can be improved by implementing longer HPLC gradients to reduce peptide co-elution or other types of chromatography in tandem, such as strong cation exchange [93]. Peptides can also be made more suitable for ESI by reducing their size by additional digestion with another protease enzyme (such as chymotrypsin or elastase) [94].

\begin{tabular}{|c|c|c|c|c|c|c|c|c|c|c|}
\hline \multicolumn{11}{|c|}{ Protein sequence coverage: $46 \%$} \\
\hline \multicolumn{11}{|c|}{ Matched peptides shown in bold red. } \\
\hline \multirow{2}{*}{\multicolumn{6}{|c|}{1 IIPPYTWYYP VRGRCALLRI LLADQGQSHTK EEWTVETVQ EGSLKASCLY }} & \multicolumn{3}{|c|}{ Database: } & & NCBInr \\
\hline & & & & & & & \multicolumn{4}{|c|}{ Score: $\quad 281$} \\
\hline \multicolumn{6}{|c|}{51 GQLPKFQDGD LTLYQSITIIL RHLGRTLGLY GKDQQEAALV DIVIIDGVEDL } & \multicolumn{5}{|c|}{ Nominal mass $\left(M_{r}\right): 23583$} \\
\hline \multirow{3}{*}{\multicolumn{6}{|c|}{$\begin{array}{l}101 \text { RCKTVSLIYT NYEVGKDDV KALPQLKPF ETLLSQIQGG KTFIVGDQIS } \\
151 \text { FADYNLLDLL LIHEVLAPGC LDAFPLLSAY VGRLSARPKL KAFLLSPETV } \\
201 \text { NLPINGNGKQ }\end{array}$}} & \multirow{2}{*}{\multicolumn{4}{|c|}{$\begin{array}{l}\text { Calculated pI: } \\
\text { Taxonomy: }\end{array}$}} & 5.43 \\
\hline & & & & & & & & & & Homo sapiens \\
\hline & & & & & & & & & & \\
\hline Query & Start - End & Observed & $\operatorname{Mr}(\operatorname{expt})$ & Irr(calc) & ppm II & I Score & Expect & Rank & U & Peptide \\
\hline$\pm \underline{103}$ & $1-12$ & 734.8924 & 1467.7703 & 1467.7585 & 8.060 & 17 & $4 e+02$ & 1 & & -.IPPYTVYYPVR.G \\
\hline$\underline{107}$ & $1-12$ & 742.9082 & 1483.8019 & 1483.7534 & 32.70 & 10 & $1.9 \mathrm{e}+03$ & 2 & & - MPPYTVVYFVVR.G + Oxidation (II) \\
\hline dg94 & $2-12$ & 669.3773 & 1336.7400 & 1336.7180 & 16.50 & 43 & 1 & $\overrightarrow{1}$ & & I. PPYTVVYFPV.G \\
\hline$t \underline{119}$ & $2-14$ & 517.6319 & 1549.8738 & 1549.8405 & 21.41 & 46 & 0.47 & $\overrightarrow{1}$ & & II.PPYTWYYFPVRGR.C \\
\hline$d \underline{1}$ & $20-30$ & 638.8449 & 1275.6753 & 1275.6281 & 37.00 & 12 & $1.8 \mathrm{e}+03$ & $\overrightarrow{6}$ & & R.IILLADQGQSFK.E \\
\hline$d \underline{81}$ & $46-55$ & 568.7684 & 1135.5222 & 1135.5696 & -41.70 & 25 & 72 & $\overline{1}$ & & K.ASCLYGQQLPK.F \\
\hline$t \underline{128}$ & $56-71$ & 628.6701 & 1882.9886 & 1882.9425 & 24.50 & 33 & 8.5 & $\overrightarrow{1}$ & U & K.FQDGDLTLYOSHTILR.H \\
\hline$d \underline{26}$ & $76-82$ & 376.2137 & 750.4129 & 750.4276 & -19.60 & 42 & 1.8 & $\overrightarrow{1}$ & & R. ILGLYGK.D \\
\hline$\llbracket \underline{171}$ & $76-101$ & 950.4882 & 2848.4428 & 2848.3913 & 18.11 & 27 & 23 & $\overrightarrow{1}$ & v & R. TLGLYGKDQQEAALVDIYVIDGVEDLR.C \\
\hline$\$ 174$ & $76-101$ & 955.8222 & 2864.4447 & 2864.3862 & 20.41 & 10 & $1.1 \mathrm{e}+03$ & 7 & U & R. TLGLYGKDQQEAALVDIYVIDGVEDLR.C + Oxidation (II) \\
\hline$\underline{d 134}$ & $83-101$ & 706.3388 & 2115.9945 & 2115.9743 & 9.580 & 26 & 40 & $\overrightarrow{1}$ & v & K.DQQEAALVDKVIDGVEDLR.C \\
\hline$\underline{135}$ & $122-141$ & 709.4037 & 2125.1893 & 2125.1531 & 17.00 & 9 & $1.9 \mathrm{e}+03$ & $\overline{6}$ & & K.ALPGQLKPFETLLSQNQQGGK.T \\
\hline
\end{tabular}

Figure 5.26: MASCOT results of a GSTp1 digest showing detection of the active site Cys47

Histidine and methionine residues are also capable of nucleophilic addition to the VSe1 $\beta$-carbon. An in silico tryptic digest of GSTp1 was performed and the $\mathrm{m} / \mathrm{z}$ of peptide ions $\left([\mathrm{M}+2 \mathrm{H}]^{+2},[\mathrm{M}+3 \mathrm{H}]^{+3}\right.$ and $[\mathrm{M}+4]^{+4}$ charges $)$ were calculated in an addition to their $193 \mathrm{Da}(\mathrm{VSe} 1-\mathrm{Cl}$ ) and $500 \mathrm{Da}(\mathrm{VSe} 1-\mathrm{Cl}+\mathrm{GSH})$ conjugates (Table 5.2). Peptides with nucleophillic side chains are highlighted in yellow. XIC's of these ions 
were studied in the enzyme digests incubated with VSe1 and compared to XIC's of the enzyme in absence of VSe1. None of the ions were uniquely detected in the VSe1-present digests, if detected at all. All of these negative results strongly suggest that VSe1 or its GSH conjugate do not covalently inhibit GSTp1. Repeating these experiments using PR1 and PR2 should be a priority for future studies and a negative result would further prove that GST and PR are detxofiying off-targets of VSes that are unrelated to the MOA. 
Table 5.2: Possible peptide ions modified by VSe1 and GSH that went undetected

\begin{tabular}{|c|c|c|c|c|c|c|c|c|c|c|c|c|c|}
\hline Sequence & Mass (Da) & Position & $\begin{array}{l}{[\mathrm{M}]^{+2}} \\
(\mathrm{Th})\end{array}$ & $\begin{array}{l}{[\mathrm{M}]^{+3}} \\
(\mathrm{Th})\end{array}$ & Cys & Met & His & $\begin{array}{c}\text { Peptide +VSe1- } \\
\mathrm{Cl}+\mathrm{GSH}[\mathrm{M}]^{+2} \\
(\mathbf{T h})\end{array}$ & $\begin{array}{l}\text { Peptide +VSel } \\
\left.\quad\left(\mathrm{M}^{+2}\right) \mathbf{( T h}\right)\end{array}$ & $\begin{array}{l}\text { Peptide +VSe1- } \\
\mathrm{Cl}\left(\mathrm{M}^{+2}\right) \text { (Th) }\end{array}$ & $\begin{array}{c}\text { Peptide +VSe1- } \\
\mathrm{Cl}+\mathrm{GSH}\left(\mathrm{M}^{+3}\right) \\
(\mathbf{T h})\end{array}$ & $\begin{array}{c}\text { Peptide +VSe } 1 \\
\left.[\mathrm{M}]^{+3} \mathbf{( T h}\right)\end{array}$ & $\begin{array}{l}\text { Peptide +VSe1- } \\
\mathrm{Cl}\left[\mathrm{M}^{+3}\right] \text { (Th) }\end{array}$ \\
\hline $\begin{array}{c}\text { TFIVGDQISFADYNLLDLLLIHEVLA } \\
\text { PGCLDAFPLLSAYVGR }\end{array}$ & 4592.4 & $142-183$ & 2297 & 1531 & 1 & 0 & 1 & 2796.707 & 2525.707 & 2489.707 & 2031.472 & 1760.472 & 1724.472 \\
\hline ALPGQLKPFETLLSQNQGGK & 2126.2 & $122-141$ & 1064 & 709.4 & 0 & 0 & 0 & 1563.58 & & & & & \\
\hline DQQEAALVDMVNDGVEDLR & 2117.0 & $83-101$ & 1059 & 706.3 & 0 & 1 & 1 & 1558.991 & 1287.991 & 1251.991 & 1206.327 & 935.3272 & 899.3272 \\
\hline AFLASPEYVNLPINGNGK & 1904.0 & 192-209 & 952.5 & 635.3 & 0 & 0 & 0 & 1452.496 & & & & & \\
\hline FQDGDLTLYQSNTILR & 1884.0 & $56-71$ & 942.5 & 628.6 & 0 & 0 & 0 & 1442.475 & & & & & \\
\hline EEVVTVETWQEGSLK & 1733.9 & $31-45$ & 867.4 & 578.6 & 0 & 0 & 0 & 1367.43 & & & & & \\
\hline YISLIYTNYEAGK & 1534.8 & $104-116$ & 767.9 & 512.3 & 0 & 0 & 0 & 1267.889 & & & & & \\
\hline MPPYTVVYFPVR & 1468.8 & 01-Dec & 734.9 & 490.3 & 0 & 1 & 1 & 1234.883 & 963.8829 & 927.8829 & 990.2552 & 719.2552 & 683.2552 \\
\hline MLLADQGQSWK & 1276.6 & $20-30$ & 638.8 & 426.2 & 0 & 1 & 1 & 1138.818 & 867.8177 & 831.8177 & 926.2118 & 655.2118 & 619.2118 \\
\hline ASCLYGQLPK & 1079.6 & $46-55$ & 540.3 & 360.5 & 1 & 0 & 0 & 1040.278 & 769.2777 & 733.2777 & 860.5185 & 589.5185 & 553.5185 \\
\hline TLGLYGK & 751.4 & $76-82$ & 376.2 & 251.1 & 0 & 0 & 0 & 876.2174 & & & & & \\
\hline LSARPK & 671.4 & 184-189 & 336.2 & 224.5 & 0 & 0 & 0 & 836.21 & & & & & \\
\hline DDYVK & 639.3 & $117-121$ & 320.1 & 213.8 & 0 & 0 & 0 & 820.1492 & & & & & \\
\hline CAALR & 533.3 & $15-19$ & 267.1 & 178.4 & 1 & 0 & 0 & 767.1432 & 496.1432 & 460.1432 & 678.4288 & 407.4288 & 371.4288 \\
\hline HLGR & 482.3 & $72-75$ & 241.6 & 161.4 & 0 & 0 & 1 & 741.6417 & & & & & \\
\hline LK & 260.2 & 190-191 & 130.6 & 87.4 & 0 & 0 & 0 & 630.5984 & & & & & \\
\hline $\mathrm{CK}$ & 250.1 & $102-103$ & 125.6 & 84.04 & 1 & 0 & 0 & 625.561 & 354.561 & 318.561 & 584.0407 & 313.0407 & 277.0407 \\
\hline GR & 232.1 & $13-14$ & 116.6 & 78.05 & 0 & 0 & 0 & 616.5702 & & & & & \\
\hline $\mathrm{Q}$ & 147.1 & $210-210$ & 74.04 & 49.69 & 0 & 0 & 0 & 574.0382 & & & & & \\
\hline
\end{tabular}


One alternative technique to observe VSe1 conjugation makes use of hydrogen/deuterium exchange on digested peptides [95]. A microfluidic reactor digests deuterated proteins on a small time-scale and residues that are exposed to ESI solvent will undergo the highest degree of exchange. A residue that is modified by VSel will theoretically undergo exchange at a significantly reduced rate relative to its unmodified version. This approach has recently been applied to studying protein-inhibitor interactions and gain a broader insight into the target protein's folding upon inhibition [96].

\subsection{Click chemistry affinity pull-downs of VSe targets}

As previously mentioned, the DIGE strategy relies on gene expression as an indirect indication of drug activity. Meanwhile, ABPP and chemical proteomics directly addresses the VSe target activity. In search of other VSe targets, a more specific pulldown strategy was adopted. Alkynated VSe probes were synthesized so that an alkyne was accessible to perform click chemistry and the chlorinated $\beta$-carbon was unmodified for target conjugation. VSe1 and MD03182 were chosen as probe scaffolds in case the nitrogen and oxygen-based rings differed in MOA (Figure 5.27). The alkynes were added to the scaffold at sites that were not required for VSe activity. Azide-biotin is introduced to the alkyne and readily undergoes a 1,3 cyclo-addition click chemistry reaction in the presence of copper(I) catalyst and ligand. This produces a triazole linker between the VSe and biotin (Figure 5.28). The complex can be isolated by affinity chromatography with streptavidin conjugated beads. On SDS-PAGE, it is expected that the VSe target band would positively correlate with increasing probe concentration (Figure 5.29). 
VSe1 Probe

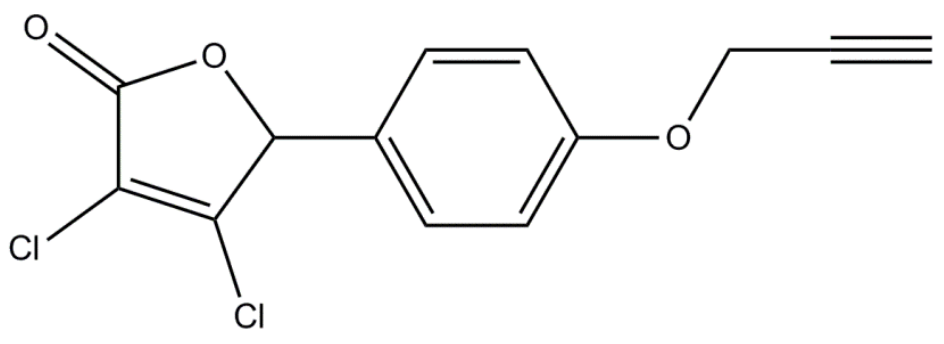

MD03182 Probe

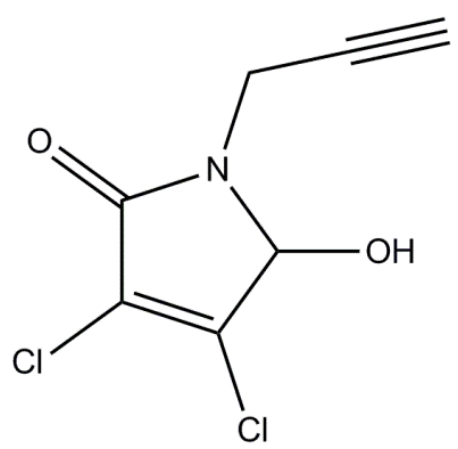

Figure 5.27: Chemical structures of the probes used for click chemistry-based ABPP

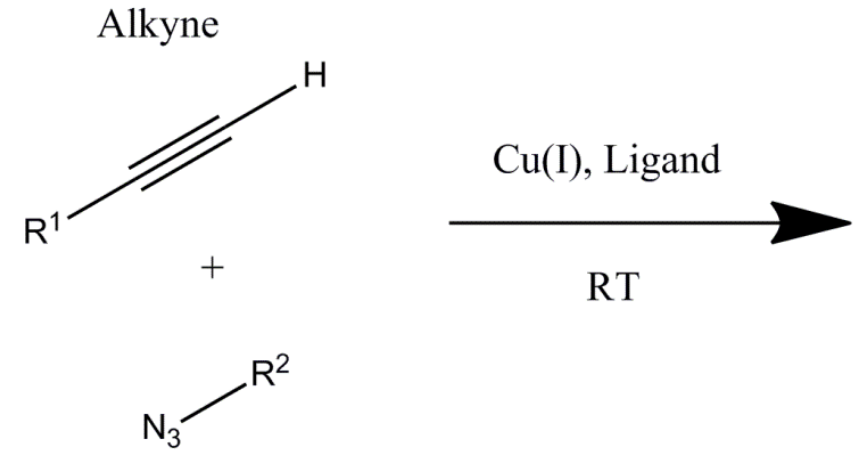

Azide

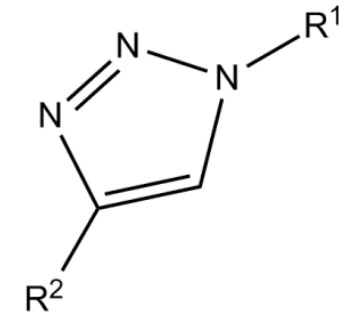

Triazole Conjugate

Figure 5.28: Copper catalyzed click chemistry cyclo-addition reaction
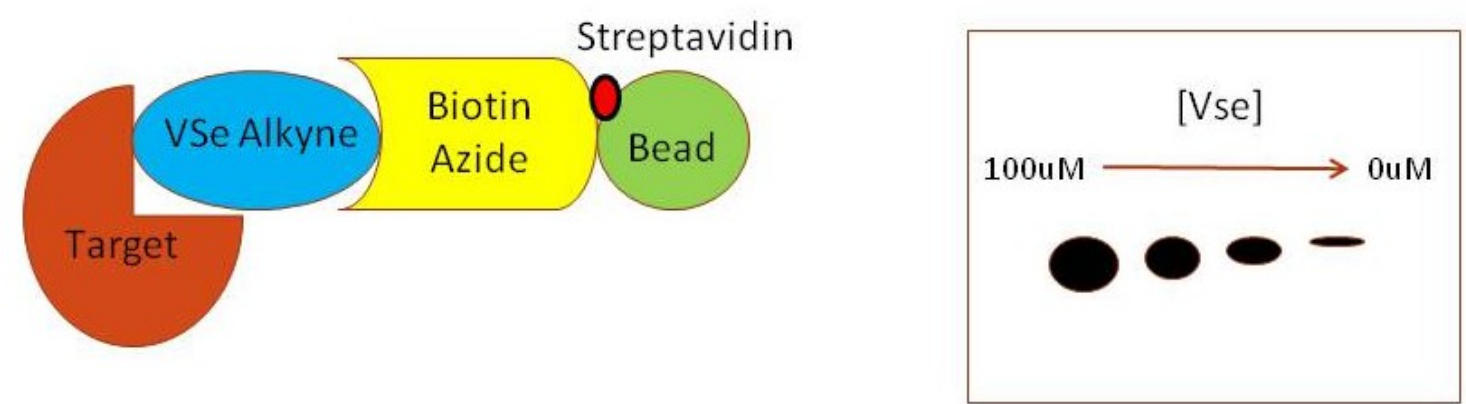

Figure 5.29: General design of a click chemistry-based ABPP target pull-down

Confluent 786-0 cells were lysed using RIPA buffer and the protein content was determined to be $30 \mathrm{mg} / \mathrm{mL}$ by Bradford assay (Biorad) [97]. VSe probes were dissolved 
in DMSO and $2 \mu \mathrm{L}$ was added to $13 \mu \mathrm{L}$ of lysate in $187 \mu \mathrm{L}$ of PBS to varying concentrations $(100 \mu \mathrm{M}, 50 \mu \mathrm{M}, 10 \mu \mathrm{M}, 1 \mu \mathrm{M}, 0.1 \mu \mathrm{M}$ and $0 \mu \mathrm{M})$. The final DMSO concentration was $0.7 \%$. The VSes were incubated with the proteins for 10 mins, 30 mins or 60 mins at $4^{\circ} \mathrm{C}$ on a rotator resulting in a total of 36 different samples. Following target incubation, the samples were spin filtered to remove non-conjugated probe that would consequently react with the biotin-azide. The proteins were collected and the following click chemistry reagents were prepared: $125 \mu \mathrm{M} \mathrm{Cu}(\mathrm{II}) \mathrm{SO}_{4}, 1.25 \mu \mathrm{M}$ tris[(1benzyl-1H-1,2,3-triazol-4-yl)methyl]amine (TBTA), $250 \mu \mathrm{M}$ azide-PEG3-biotin in water and $0.5 \mathrm{mM}$ ascorbic acid in PBS. $80 \mu \mathrm{L}$ of the $\mathrm{CuSO}_{4}$ solution and $20 \mu \mathrm{L}$ of the ligand solution were added in succession and the click chemistry reaction was allowed to proceed for 60 mins at room temperature. The samples were spin filtered once again to remove unreacted biotin that would saturate the streptavidin-sepharose (Sigma) beads down-stream and potentially limit the target's binding. The bead slurry was washed three times in an equal volume of PBS and pelleted with 5 min centrifugations at $2000 \mathrm{rpm}$ and resuspended. $163 \mu \mathrm{L}$ of bead slurry was added to each lysate sample and incubated for 30 mins at $4^{\circ} \mathrm{C}$. The beads were washed three times with $200 \mu \mathrm{L}$ PBS to remove nonspecific proteins and pelleted. Target proteins were eluted from the beads by boiling in 30 $\mu \mathrm{L} 2 \mathrm{X}$ Laemmlli buffer (5 $\mu \mathrm{L}$ 10X Laemmlli buffer with $12.5 \mu \mathrm{L} 4 \mathrm{X}$ DTT and $38 \mu \mathrm{L}$ of lysis buffer) for 5 mins and the supernatant was analyzed by SDS-PAGE. The gels were stained with Coomassie stain solution (0.25\% Coomassie R250 Blue, 50\% MeOH, 10\% acetic acid) for $1 \mathrm{hr}$ and destained five times in destain solution (16.5\% $\mathrm{MeOH}, 5 \%$ acetic acid) for 30 mins. The gels were imaged using an AlphaImager HP (Proteinsimple). 


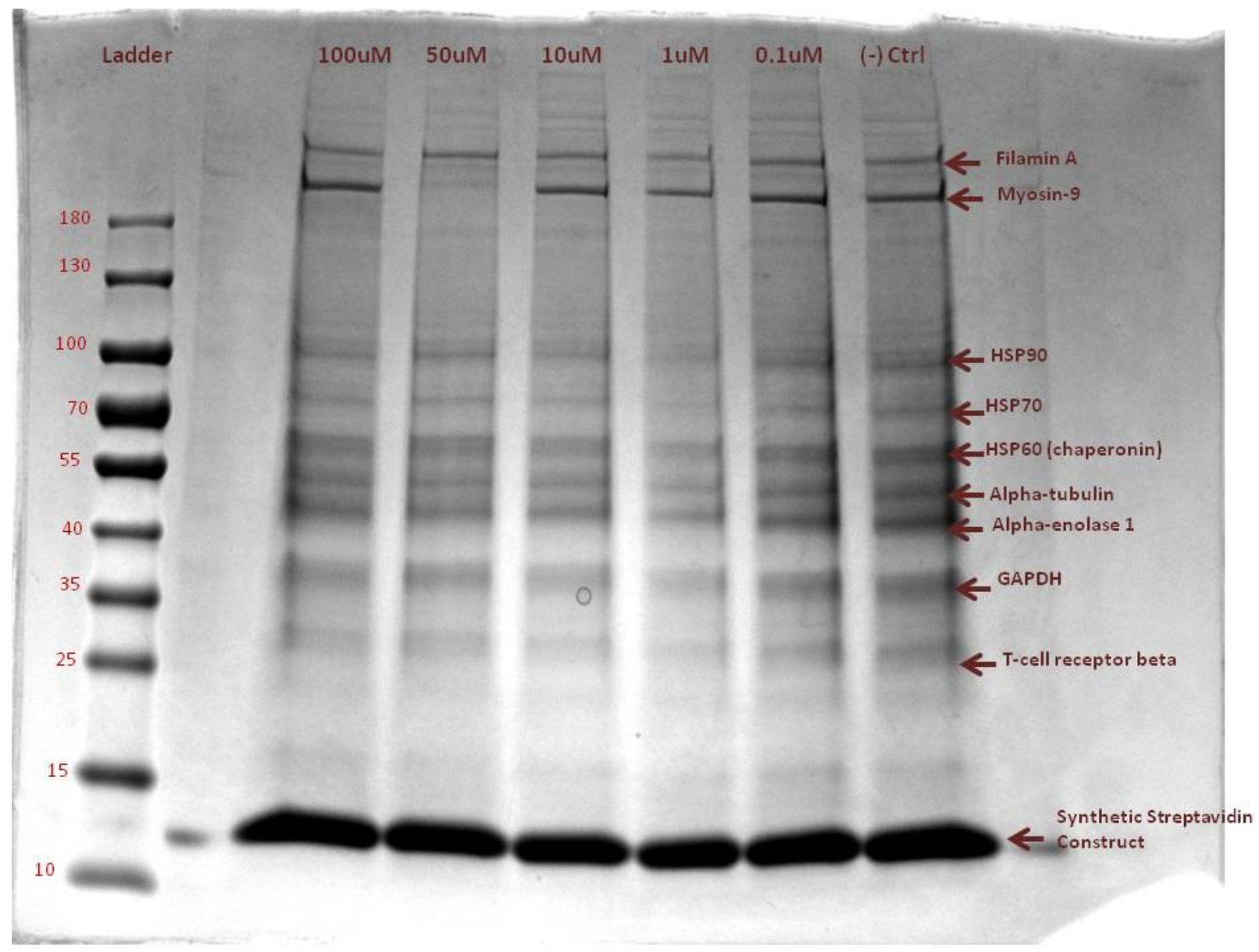

Figure 5.30: Proteins pulled down from 786-0 lysate using the MD03182-based probe for 60 mins

The gel image shown above (Figure 5.30) is representative of all samples, indicating that proteins readily bound to the streptavidin-beads in a non-specific manner. It was hypothesized that these proteins were naturally biotinylated because they were putatively pulled-down. They could be depleted by treating lysate with streptavidin beads prior to VSe treatment. However, this was not the case because the most intensely stained bands were identified as naturally highly abundant proteins, such as GAPDH, tubulin and myosin. 
The non-specific proteins could have been masking the staining of the VSe target so the bead washing step could be optimized in a variety of ways [98]. As a troubleshooting experiment, 786-0 cell lysate was incubated with streptavidin beads (without the click chemistry and biotin steps) and were washed with various buffers in order: PBS, 1\% Triton lysis buffer (LB), $\mathrm{pH} 4$ acetate buffer followed by $\mathrm{pH} 8.4$ borate buffer (A/B), high salt (HS) $500 \mathrm{mM} \mathrm{NaCl}$, A/B washes followed by HS washes, 2\% SDS and $0.5 \%$ digitonin (digi). Lysate without bead purification was used as a comparison for poor washing. SDS-PAGE revealed that all tested buffers effectively removed non-specific protein (Figure 5.31), including PBS which contradicts the previous results. The lysate without bead purification appears very similar to the banding patterns observed in Figure 5.29, thus confirming ineffective washing during the ABPP experiment due to a lack of vigorous vortexing.

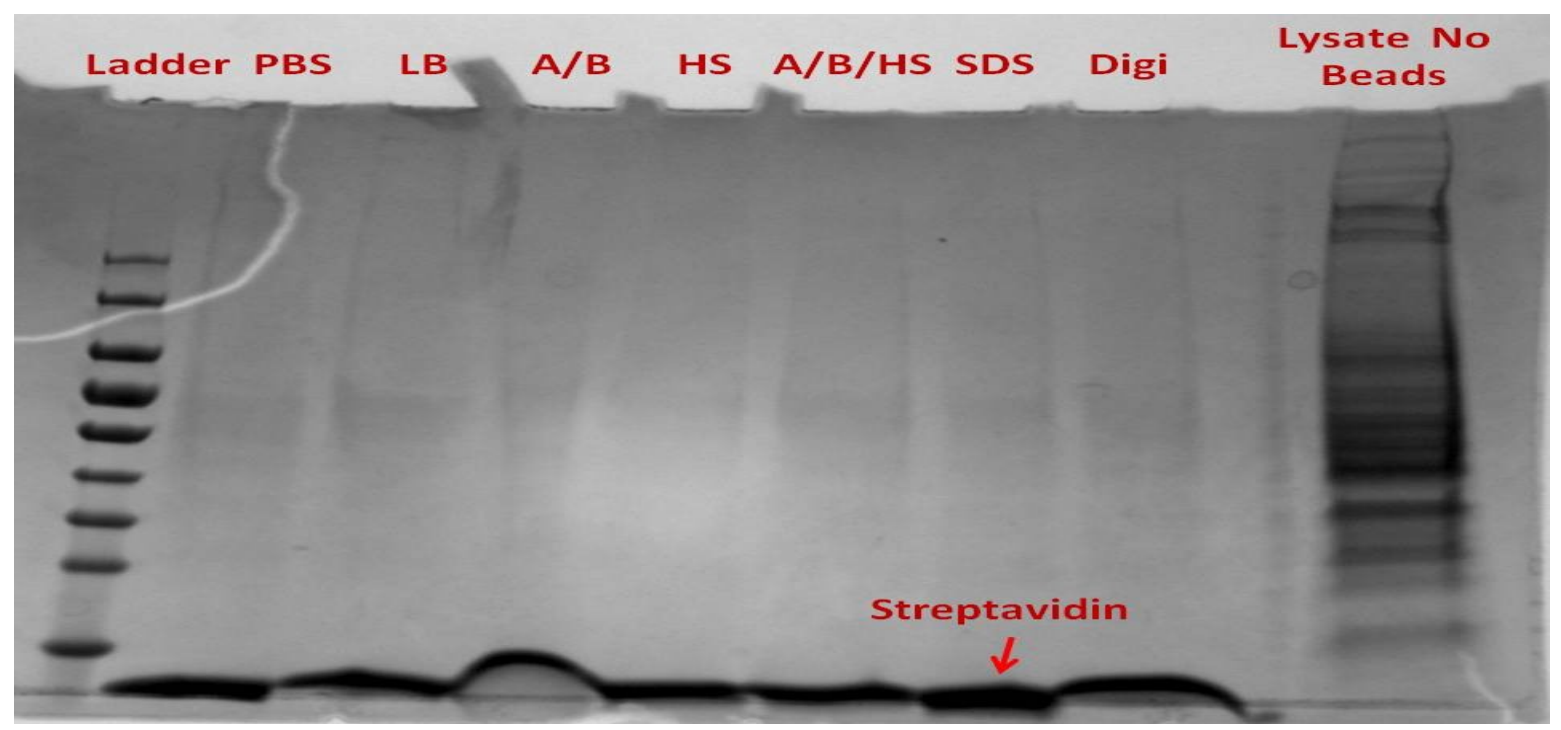

Figure 5.31: Qualitative assessment of various bead washing conditions all show efficacy

The click chemistry-based pull-down was repeated using the similar conditions with a few exceptions. Probes were incubated with lysate for 60 mins instead of 10 or 30 
mins to ensure protein conjugation was complete. The temperature of the lysate incubation was changed from $4^{\circ} \mathrm{C}$ to $37^{\circ} \mathrm{C}$ to mimic in vitro conditions. The click chemistry stock reagents were prepared at higher concentrations: $1 \mathrm{mM} \mathrm{Cu}(\mathrm{II}) \mathrm{SO}_{4}, 2.5$ $\mathrm{mM}$ TBTA, and $1 \mathrm{mM}$ sodium ascorbate ligand individually in $\mathrm{H}_{2} \mathrm{O}$ and $7 \mathrm{mM}$ biotin PEG-3 azide in DMSO. $2 \mu \mathrm{L}$ of each click chemistry reagent was added to $50 \mu \mathrm{L}$ of spin filtered protein prior to the final addition of $\mathrm{Cu}(\mathrm{II}) \mathrm{SO}_{4}$ solution. Streptavidin beads were washed with PBS containing protease inhibitors three times with vortexing. The resultant gel images show no specific bands for either probe (Figure $5.32 \& 5.33$ ). The only observed band was non-specific since it was detected in the negative control sample and was likely streptavidin due to its similar positioning with the band in Figure 5.31.

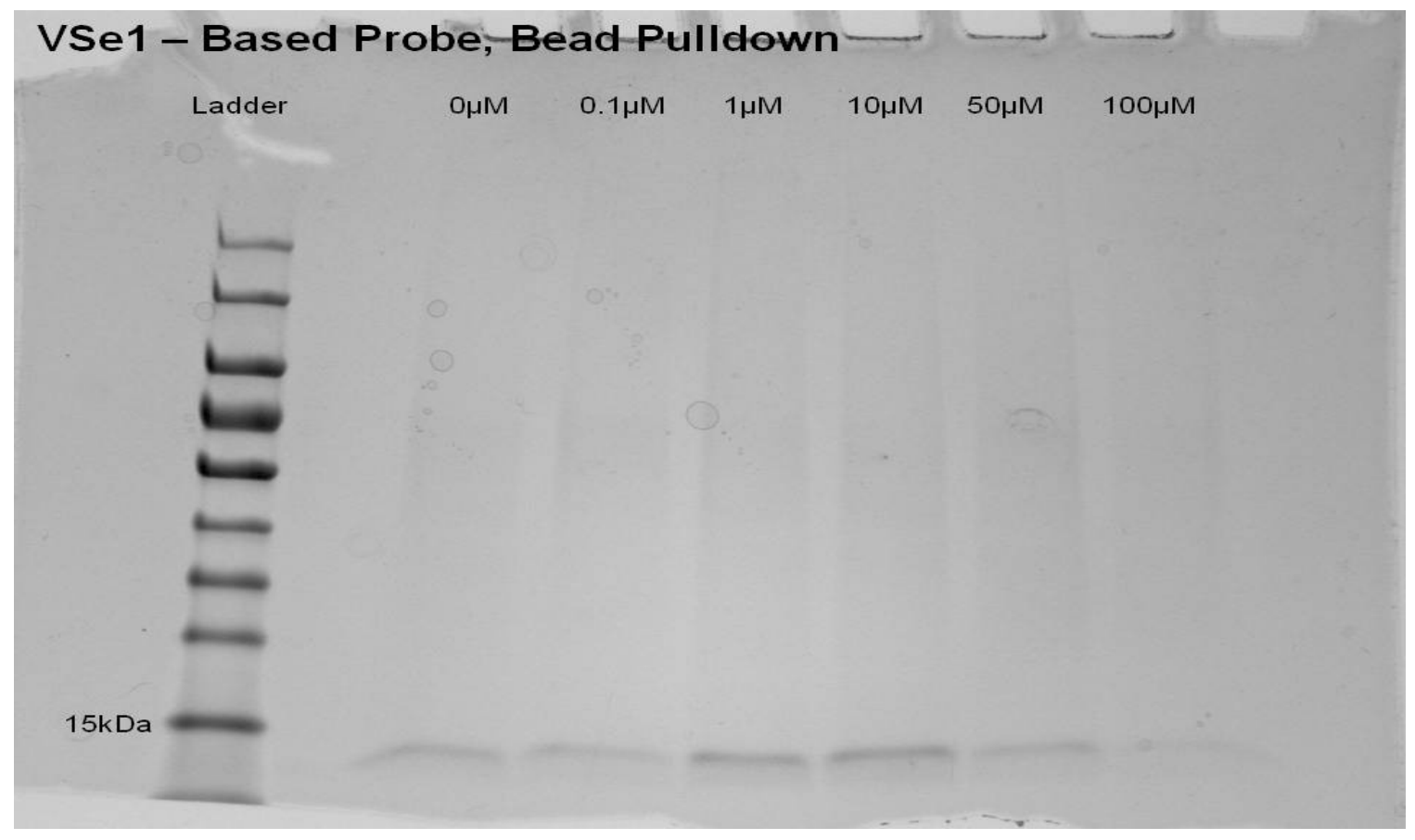

Figure 5.32: VSe1-based probe target pull-down from 786-0 cell lysate using streptavidin-linked sepharose beads 


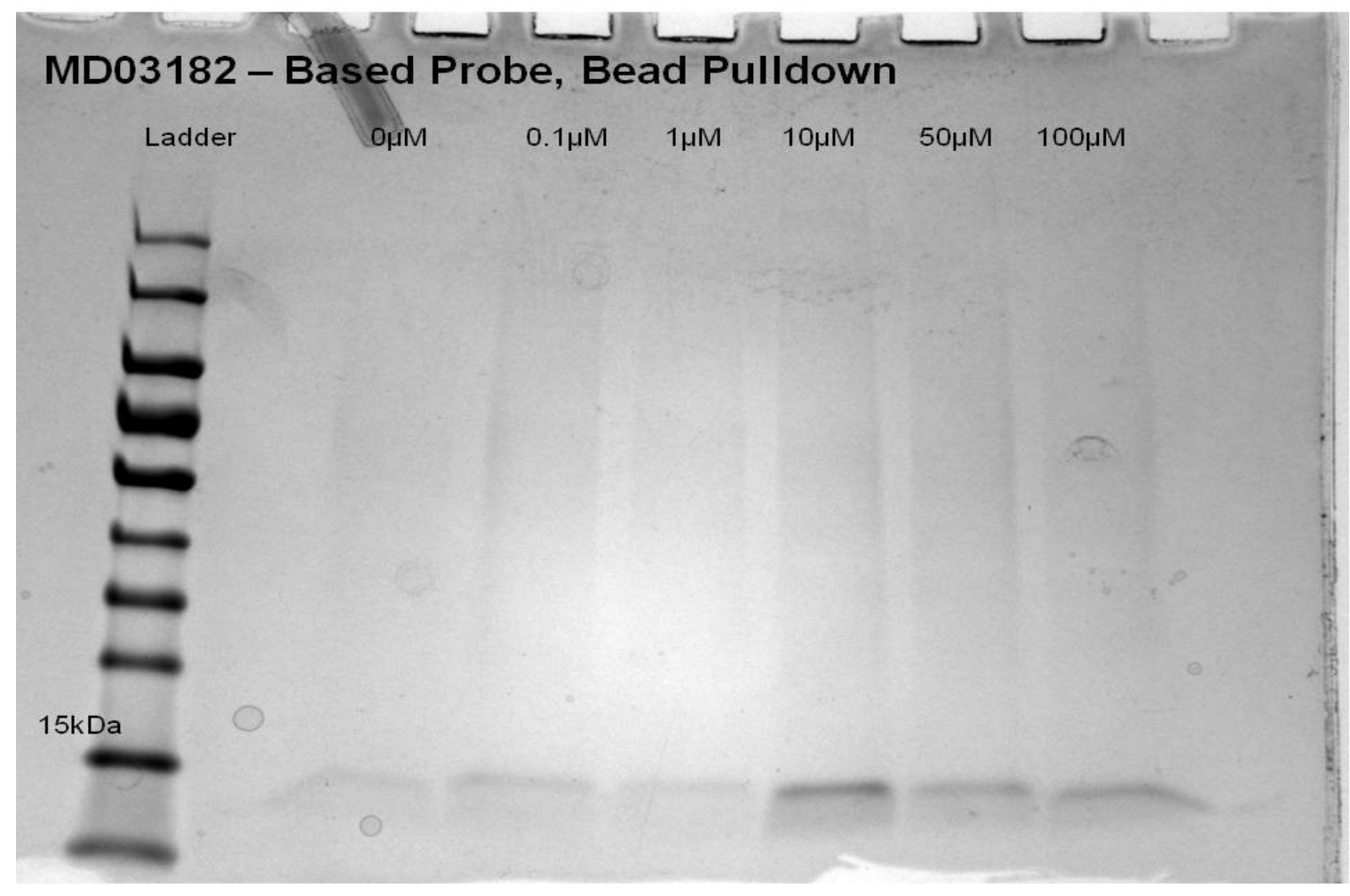

Figure 5.33: MD03182-based probe target pull-down from 786-0 cell lysate using streptavidin-linked sepharose beads

To circumvent these troubleshooting issues surrounding the sepharose beads, another click chemistry-oriented strategy was applied. As Peszacki et al. have demonstrated, the biotin-azide can be substituted with a fluorescent rhodamine-azide tag [99] (Figure 5.34)

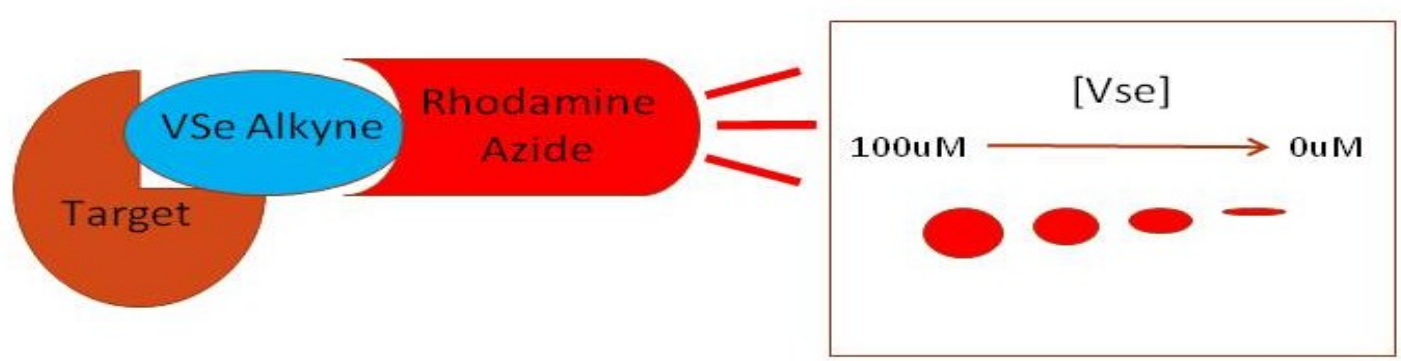

Figure 5.34: General design of a click chemistry-based pull-down using rhodamine fluorescent tagging 
The same probes were incubated with $786-0$ cell lysate for 60 mins at $37^{\circ} \mathrm{C}$ for target modification. The click chemistry reaction was performed the same way as in the last bead-based pull-down experiment except the rhodamine-azide (Sigma) replaced the biotin-azide and was dissolved in DMSO at a concentration of $250 \mu \mathrm{M}$ and $2 \mu \mathrm{L}$ was added to the proteins. Gel images were obtained by fluorescence analysis using an FMBio III gel scanner (Hitachi) with excitation and emission wavelengths of $542 \mathrm{~nm}$ and $605 \mathrm{~nm}$ respectively (Figure 5.35).

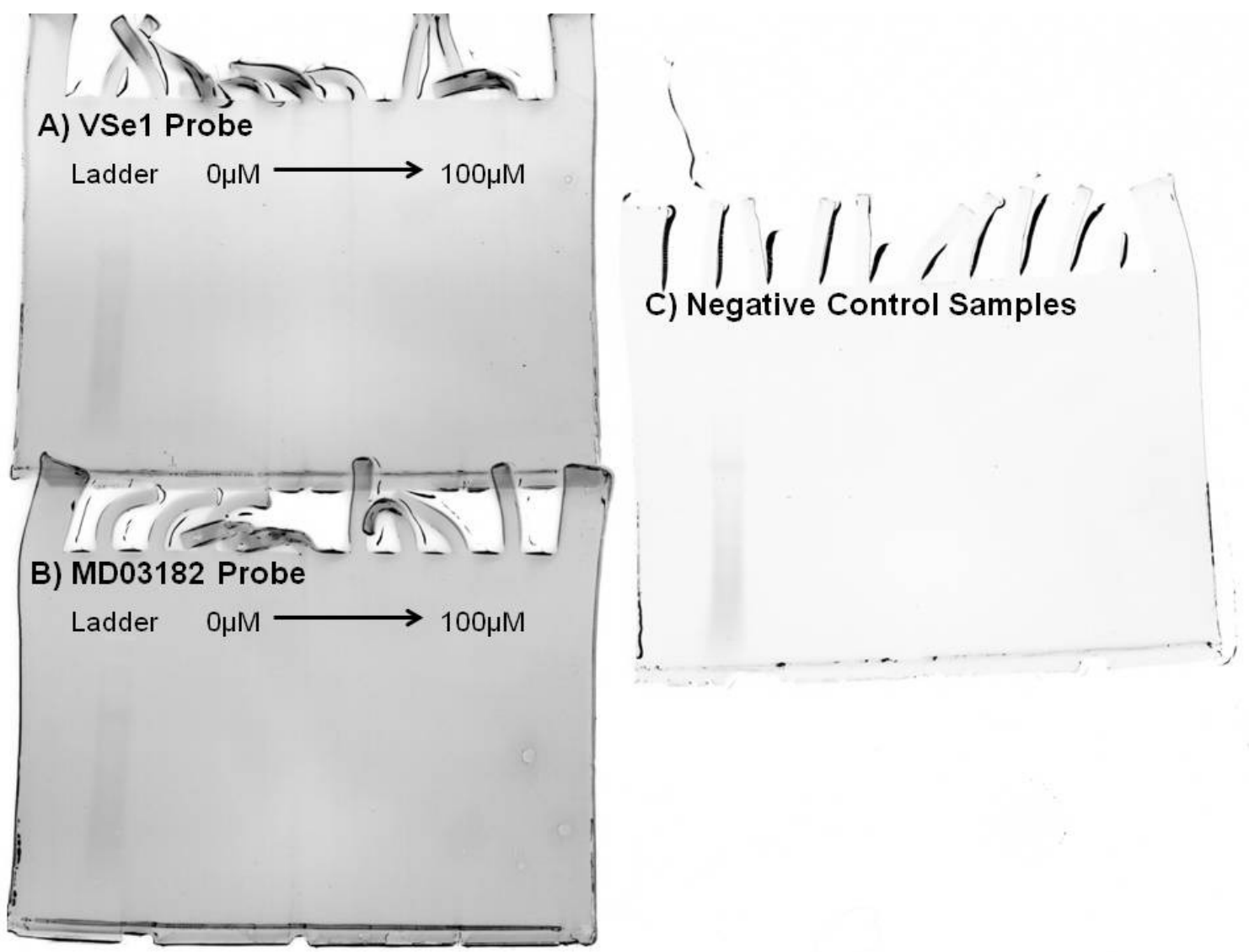

Figure 5.35: ABPP VSe target identification from 786-0 cell lysate using rhodamine fluorescent tag

The gel images showed no protein bands that were conjugated to their probes with a minimal amount of fluorescence detected from the protein ladder. These results further 
support the hypothesis that VSes are not covalent inhibitors, thus the probe is removed during the first spin filtration step. It could also mean that the click chemistry reaction is not occurring and the targeted proteins are not labeled. Repetitions of this experiment should include a positive control that ensures the click chemistry reaction is taking place. A suitable positive control would be fluorophosphonate-PEG probe clicked to a rhodamine tag to detect serine hydrolase target enzymes [99].

Even if fluorescent protein bands were to be detected using this technique, a few challenges would still need to be overcome. One drawback to this method is that excised protein bands will contain putative proteins in addition to the fluorescent target, making MS-identification more difficult. Longer HPLC gradients will reduce peptide co-elution and improve the mass spectrometer's ability to detect lower-abundance target peptides. Another strategy involved pooling the three samples containing the lower probe concentrations for in-gel digestion and proteins that are less abundant relative to the three pooled samples associated with higher probe concentrations could serve as an indicator for specificity. Another way to indicate target specificity would be to perform the experiment in parallel with non-alkynated probes that would not react with the azidefluorophore, thus bands disappearing in these gels would be specific targets. Finally, it has been suggested that the rhodamine-azide strategy selectively works in situ rather than in cell lysate. Future experiments will also need to test the probing of whole cells in case cell lysis and subsequent proteolysis interferes with VSe protein conjugation [38].

If challenges continue with click chemistry-based ABPP, another method to link probes to a detectable tag is through photo-activation. Sherratt et al. used a photo-reactive probe that covalently crosslinks to its nearest interacting partner upon irradiation with UV 
light. The target can be visualized by a reporter fluorophore and this is useful for monitoring non-covalent drug-target interactions [100]. One ABPP strategy that could also act as an alternative technique is on-bead tryptic digestion. VSe probes covalently bound to sepharose beads can modify the protein target's active site. After washing away non-specific proteins, the enriched proteins are tryptically digested and the resultant peptides can be identified by LC-MS [101].

\subsection{Conclusions}

Overall, the proteomic techniques mentioned in this chapter were unsuccessful in elucidating the VSe MOA. Extensive troubleshooting of these methods is required to overcome issues that involve DIGE IEF, target purification and click chemistry. All of the results presented in this chapter lead to the hypothesis that VSes do not covalently inhibit their target; non-covalent interactions could play a role. Alternatively, other aforementioned proteomic and ABPP approaches could prove to be more helpful. It is recommended that future VSe studies put a greater emphasis on target identification including PK-M2, annexinA2, and PR inhibition assays. The knowledge gained from these experiments, including identification of the activated effector pathways and the active site environment would present numerous opportunities in SAR, and the study of toxic effects induced by unintended off targets. 


\section{Chapter: Concluding Remarks}

Small molecules provide a novel approach to improve the propagation of attenuated viral infection in resistant cells. VSe1 was determined to be unsuitable for in vivo applications due to poor physiochemical properties, thus creating a demand for robust analysis of analogues during SAR and hit expansion. LC-MS techniques applied enhanced full mass, product, NL and MRM scanning modes to screen a library of VSes for more desirable properties. Promisingly, new lead compounds were more tolerated and more stable in plasma with substantial retained activity. LC-MS was used to study the PK and metabolomic behaviour of the new lead VSes which emphasized the importance of tumour penetrance and glutathione stability. LC-MS approaches were also used to monitor the stability of VSes under various storage and administration conditions that will improve reproducibility amongst pre-clinical experiments. Proteomic studies used MS-based and gel-based approaches to investigate the VSe MOA which support VSes acting as non-covalent inhibitors; yet the intracellular target remained unknown. The knowledge gained from the experiments described in this dissertation will ultimately prove to be useful in improving the application of VSe technology towards vaccine production and OVt. 


\section{References}

[1] Hammer, S. M. 2015. Viral Replication - Course Lecture. [Online]. Available: http://www.columbia.edu/itc/hs/medical/pathophys/id/2005/MIDHammerViralRepColor.pdf.

[2] Barre-Sinoussi, F., Ross, L.A., Delfraissy, J.F. 2013. Past, present and future: 30 years of HIV research. Nat. Rev. Microbiol., 11, 877-883.

[3] Center for Disease Control and Prevention. 2015. HIV/AIDS. [Online]. Available: http://www.cdc.gov/hiv/.

[4] Chowell, G., Nishiura, H. 2014. Transmission dynamics and control of Ebola virus disease (EVD): a review. BMC Med., 12, 196-205.

[5] Guidotti, L.G., Chisari, F.V. 2001. Noncytolytic control of viral infections by the innate and adaptive immune response. Annu. Rev. Immunol., 19, 65-91.

[6] Goodburn, S., Didcock, L., Randall, R.E. 2000. Interferons: Cell signalling, immune modulation, antiviral responses and virus countermeasures. J. Gen. Virol. 81, 2341-2364.

[7] Okumura, A., Lu, G., Pitha-Rowe, I. et al. 2006. Innate antiviral response targets HIV-1 release by the induction of ubiquitin-like protein ISG15. Proc. Natl. Acad. Sci. U. S. A., 103 (5), 1440-1445.

[8] Sadler, A.J., Williams, B.R.G. 2008. Interferon-inducible antiviral effectors. Nat. Rev. Immunol., 8, 559-568.

[9] Yin, Y., Manoury, B., Fahraeus, R. 2003. Self-inhibition of synthesis and antigen presentation by Epstein-Barr virus-encoded EBNA1. Science, 301,1371-1374.

[10] Hansasuta, P., Dong, T., Thananchai, H. et al. 2004. Recognition of HLA-A3 and HLA-A11 by KIR3DL2 is peptide-specific. Eur. J. Immunol., 34, 1673-1679.

[11] Iannello, A., Debbeche, O., Martin, E. A. et al. 2006. Viral strategies for evading antiviral cellular immune responses of the host. J. Leukoc. Biol. 79(1), 16-35.

[12] Diallo, J-S., Boddy, C.N., Dornan, M. et al. 2015. Compositions and methods for viral sensitization. USA Patent Application. 08924773US

[13] Ulmer, J.B., Valley, U., Rappuoli, R. 2006. Vaccine manufacturing: challenges and solutions. Nat. Biotechnol., 24(11), 1377-1383. 
[14] Patriarca, P. 2007. Use of cell lines for the production of influenza virus vaccines : an appraisal of technical, manufacturing, and regulatory considerations. World Heal. Organization Reports, 1-12.

[15] Barrett, P.N., Howard, M.K., Kistner, O. et al. 2015. Vero cell platform in vaccine production: moving towards cell culture-based viral vaccines. Expert Rev. Vaccines, 5, 1-19.

[16] Litvinov, S. 1987. Acceptability of cell subtrates for production of biologicals," World Heal. Organization Reports, 1-29.

[17] Whitford, W. G., Fairbank, A. 2011. Considerations in scale-up of viral vaccine production. Bioprocess Int., 9, 16-28.

[18] Liu, M.A. 2010. Gene-based vaccines: recent developments. Curr. Opin. Mol. Ther., 12, 86-93.

[19] Canadian Cancer Society. 2015. Cancer statistics at a glance.[Online]. Available: http://www.cancer.ca/en/cancer-information/cancer-101/cancer-statistics-at-aglance/?region $=$ on.

[20] Bertam, J.S. 2000. The molecular biology of cancer. Mol. Aspects Med., 21(6), 167-223.

[21] Salgia, R., Li, J.L., Ewaniuk, D.S. et al. 1997. BCR/ABL induces multiple abnormalities of cytoskeletal function. J. Clin. Invest., 100(1),46-57.

[22] Rajalingam, K., Schreck, R., Rapp, U. P. et al. 2007. Ras oncogenes and their downstream targets. Biochim. Biophys. Acta - Mol. Cell Res., 1773(8), 1177-1195.

[23] Hunter, T., Pines, J. 1994. Cyclins and cancer II: cyclin D and CDK inhibitors come of age. Cell, 79, 573-582.

[24] Packham, G., Cleveland, J.L. 1994. C-Myc and apoptosis. Biochim. Biophys. Acta., 1242, 11-28.

[25] Aurelian, L. 2013. Oncolytic virotherapy: the questions and the promise. Oncolytic Virotherapy, 2, 19-29.

[26] Shashkova, E.V., May, S.M., Doronin, K. et al. 2009. Expanded anticancer therapeutic window of hexon-modified oncolytic adenovirus. Mol. Ther., 17(12), $2121-2130$. 
[27] Pan, W., Bodempudi, V., Esfandyari, T. et al. 2009. Utilizing Ras signaling pathway to direct selective replication of herpes simplex virus-1. PLoS One, 4(8), $2-10$.

[28] Cawood, R., Chen, H. H., Carroll, F. et al. 2009. Use of tissue-specific microRNA to control pathology of wild-type adenovirus without attenuation of its ability to kill cancer cells. PLoS Pathog., 5(5), 1-10.

[29] Dornan, M., Krishan, R., Macklin, A. et al. 2015. First-in-class small molecule potentiators of tumor-specific oncolytic virus infection. Manuscript in Preparation. 1-25.

[30] United States Institutes of Health. 2015. Efficacy and safety study on OncoVEXGM-CSF compared to GM-CSF in melanoma. Clinical Trial NCT0076970.

[31] United States Institutes of Health. 2015. MG1 Maraba/MAGE-A3, with and without Adenovirus vaccine, with transgenic MAGE-A3 insertion in patients with incurable MAGE-A3-expressing solid tumours (I214)," Clinical Trial NCT0228581.

[32] Stojdl, D.F., Lichty, B.D., TenOever, J.M. et al. 2003. VSV strains with defects in their ability to shutdown innate immunity are potent systemic anti-cancer agents. Cancer Cell, 4, 263-275.

[33] Lichty, B.D., Power, A.T., Stojdl, D.F. et al. 2004. Vesicular stomatitis virus: Reinventing the bullet. Trends Mol. Med., 10(5), 210-216.

[34] Diallo, J-S., Le Boeuf, F., Lai, F. et al. 2010. A high-throughput pharmacoviral approach identifies novel oncolytic virus sensitizers. Mol. Ther., 18(6), 11231129.

[35] Diallo, J-S. Boddy, C.N., Dornan, M. 2015. Compositions and methods for enhancing oncolytic virus efficacy. United States Patent Application. Number Not Issued.

[36] Wilcken, B., Wiley, V., Carpenter, K. 2003. Screening newborns for inborn errors of metabolism by tandem mass spectrometry. N. Engl. J. Med., 348, 2304-2312.

[37] Johnson, P.V., Hodyss, R., Beauchamp, J.L. 2014. Ion funnel augmented Mars atmospheric pressure photoionization mass spectrometry for in situ detection of organic molecules. J. Am. Mass Spectrom. Soc., 25(11), 1832-1840.

[38] Turetsky, M. R., Manning, S.W., Wilder, R.K. 2004. Dating recent peat deposits. Wetlands, 24(2), 324-356. 
[39] Griffiths, I.W. 1997. J. J. Thomson - The centenary of his discovery of the electron and of his invention of mass spectrometry. Rapid Commun. Mass Spectrom., 11(1), 2-16

[40] Boggess, B. 2015. Ionization Modes. University of Notre Dame Mass Spectrometry and Proteomics Facility. [Online]. Available: http://massspec.nd.edu/instruments-and-analyses/ionization-modes/.

[41] Aebersold, R., Mann, M. 2003. Mass spectrometry-based proteomics. Nature, 422, 198-207, 2003.

[42] Ho, C.S., Lam, C.W., Chan, M.H.M. 2003. Electrospray ionisation mass spectrometry: principles and clinical applications. Clin. Biochem. Rev., 24, 3-12.

[43] Waters. 2015. Common Ionization. [Online]. Available: http://www.waters.com/waters/en_US/CommonIonization/nav.htm?cid=10073251.

[44] Smith, J.C. 2013. Putting technologies together. Course Lecture - Carleton University CHEM 5109. [Online]. Available:

https://culearn.carleton.ca/moodle/pluginfile.php/398266/mod_resource/content/0/ CHEM 4304-5109 - Lecture 5 - Feb 52013 - Putting technologies together.pdf.

[45] Douglas, D.J. 2009. Linear quadrupoles in mass spectrometry. Mass Spectrom. Rev., 28(6), 937-960.

[46] Griffiths, W. J., Wang, Y. 2009. Mass spectrometry: from proteomics to metabolomics and lipidomics. Chem. Soc. Rev., 38, 1882-1896.

[47] Guilhaus, M., Selby, D., Mlynski, V.M. 2000. Orthogonal acceleration time-offlight mass spectrometry. Mass Spectrom. Rev., 19(2), 65-107.

[48] Wasslen, K.V. 2014. Trimethylation enhancement using diazomethane (TrEnDi): A novel technique to enhance the sensitivity of MS-based analyses of biological molecules. Masters Thesis. Carleton University.

[49] Douglas, D., Frank, A., Mao, D. et al. 2005. Linear ion traps in mass spectrometry. Mass Spectrom. Rev., 24, 1-29.

[50] Applied Biosystem. 2010. 4000 QTRAP ${ }^{\circledR}$ System Hardware Guide. AB Sciex. [Online]. Available: http://sciex.com/Documents/Downloads/Literature/4000qtrap-hardware-guide.pdf

[51] Wiza, J. L. 1979. Microchannel plate detectors. Nucl. Instruments Methods, 162(13), 587-601. 
[52] Seah, M. P. 1989. Channel electron multipliers: quantitative intensity measurement - efficiency, gain, linearity and bias effects. J. Electron Spectros. Relat.

Phenomena, 50(1), 137-157.

[53] Palzs, B., Suhal, S. 2005. Fragmentation pathways of protonated peptides. Mass Spectrom. Rev., 24, 508-548.

[54] Smith, J.C. 2005. Mass spectrometry-based proteomics: non-covalent interactions and protein identification. $\mathrm{PhD}$ Thesis. York University.

[55] Hunter, E.P.L., Lias, S.G. 1998. Evaluated gas phase basicities and proton affinities of molecules: an update. J. Phys. Chem. Ref. Data, 27(3), 413-656.

[56] Promega Corporation. 2013. Sequencing Grade Modified Trypsin. [Online]. Available: http://www.promega.ca/ /media/files/resources/protocols/product information sheets $/ \mathrm{n} /$ sequencing grade modified trypsin protocol.pdf.

[57] Wilm, M., Shevchenko, A., Houthaeve, T. et al. 1996. Femtomole sequencing of proteins from polyacrylamide gels by nano-electrospray mass spectrometry. Nature, 379, 466-469.

[58] Millipore. 2012. Amicon ${ }^{\circledR}$ Ultra-4 Centrifugal Filter Devices. [Online]. Available: http://www.emdmillipore.com/CA/en/product/Amicon-Ultra- $0.5 \mathrm{~mL}-$ Centrifugal-Filters-for-DNA-and-Protein-Purification-andConcentration,MM_NF-C82301\#overview.

[59] Savas, J.N., De Wit, J., Comoletti, D. 2014. Ecto-Fc MS identifies ligand-receptor interactions through extracellular domain Fc fusion protein baits and shotgun proteomic analysis. Nat. Protoc., 9(9), 2061-2074.

[60] Erguetta, T. 2006. Trends in mass spectrometry: Better tools, approaches and information. Pharm. Technol. Eur. 1-2, 2006.

[61] Gumustas, M., Krubanoglu, S., Uslu, B. 2013. UPLC versus HPLC on drug analysis: advantages, application and their validation parameters. Chromatographia, 76(21-22), 1365-1427.

[62] Lim, C., Lord, G. 2002. Current developments in LC-MS for pharmaceutical analysis. Biol. Pharm. Bull., 25(5), 547-557.

[63] Schulze, W.X., Usadel, B. 2010. Quantitation in mass-spectrometry-based proteomics. Annu. Rev. Plant Biol., 61, 491-516.

[64] Armbruster, D.A., Pry, T. 2008. Limit of blank, limit of detection and limit of quantitation.," Clin. Biochem. Rev., 29, 49-52. 
[65] Meng, M. Bennett, P.K. 2012. LC-MS in Drug Bioanalysis. Springer.

[66] Fiehn, O., Weckwerth, W. 2015. Mass Spectrometry: Quantitation. Springer.

[67] Hughes, J. P., Rees, S., Kalindjian, B. 2011. Principles of early drug discovery. Br. J. Pharmacol., 162(6), 1239-49.

[68] Kerns, E.H., Di, L. 2008. Drug-like properties: concepts, structure design and methods. Elsevier Inc.

[69] Jancova, P., Anzenbacher, P., Anzenbacherova, E. 2010. Phase II drug metabolizing enzymes. Biomed. Pap. Med. Fac. Univ. Palacky Olomouc, 154(2), $103-116$.

[70] Scripps Center for Metabolomics. 2015. Metlin metabolite search. [Online]. Available: https://metlin.scripps.edu/index.php.

[71] Schramm, G., Surmann, E-M., Wiesberg, S. 2010. Analyzing the regulation of metabolic pathways in human breast cancer. BMC Med. Genomics, 3(39), 1-10.

[72] Perroud, B., Lee, J., Valkova, N. et al. 2006. Pathway analysis of kidney cancer using proteomics and metabolic profiling. Mol. Cancer, 5(64), 1-47.

[73] Lomenick, B., Olsen, R.W., Huang, J. 2011. Identification of direct protein targets of small molecules. ACS Chem. Biol., 6(1), 34-46.

[74] Lomenick, B., Hao, R., Jonai, N. et al. 2009. Target identification using drug affinity responsive target stability (DARTS). Proc. Natl. Acad. Sci. U. S. A., 106(51), 21984-21989.

[75] Berg, J. 2012. Biochemistry. Macmillan.

[76] Cenre for Genomic Sciences. 2015. Two dimensional gel electrophoresis (2D). University of Hong Kong [Online]. Available: http://cgs.hku.hk/portal/index.php/2ddige.

[77] Lokamani, I., Looi, M-L., Ali, S.A.M. 2014. Gelsolin and ceruloplasmin as potential predtive biomarkers for cervical cancer by 2D-DIGE proteomics analysis. Pathol. Oncol. Res. 20(1), 119-129.

[78] Barrack, S. 2013. Biochemical biomarkers in Alzheimer's disease. iMedPub.

[79] Muroi, M., Kazami, S., Noda, K. 2010. Application of Proteomic Profiling Based on 2D-DIGE for Classification of Compounds According to the Mechanism of Action. Chem. Biol., 17(5), 460-470. 
[80] EMD Biosciences. 2005. Subcellular proteome extraction kit. [Online]. Available: http://www.emdmillipore.com/CA/en/product/ProteoExtract\%C2\%AESubcellular-Proteome-Extraction-Kit,EMD_BIO-539790-1KIT?bd=1

[81] Bio-Rad. 2015. Bio-rad protein assay. [Online]. Available: http://www.bio$\mathrm{rad} . \mathrm{com} / \mathrm{en}-\mathrm{ca} / \mathrm{product} / \mathrm{bio}-\mathrm{rad}-$ protein-assay

[82] Proteomics and Mass Spectrometry Facility. 2015. Coomassie blue staining protocol. Cornell University.[Online]. Available:

http://www.biotech.cornell.edu/sites/default/files/uploads/Documents/Proteomics_ protocols/Protocol 05_Coomassie.pdf. [Accessed: 27-Mar-2015].

[83] National Chiao Tung University. 2015. CELLO: subcellular localization predictive system. [Online]. Available: http://cello.life.nctu.edu.tw/.

[84] Yin, L., Wang, X., Luo, C. 2013. The value of expression of M2-PK and VEGF in patients with advanced gastric cancer. Cell Biochem. Biophys., 67(3), 1033-1039.

[85] Tonnus, C., Neupert, G., Mellinger, M. 2006. Colorectal cancer screening by noninvasive metabolic biomarker fecal tumor M2-PK. World J. Gastroenterol., 12(43),7007-11.

[86] Wang, C-Y., Lin, C-F. 2014. Annexin A2: its molecular regulation and cellular expression in cancer development. Dis. Markers, 1-10.

[87] Dziduscko, A., Ozbun, M.A. 2013. Annexin A2 and S100A10 regulate human papillomavirus type 16 entry and intracellular trafficking in human keratinocytes. J. Virol., 87(13), 7502-7515.

[88] Thermo Scientific. 2015. Pierce BCA protein assay kit. [Online]. Available: https://tools.lifetechnologies.com/content/sfs/manuals/MAN0011430_Pierce_BCA _Protein_Asy_UG.pdf.

[89] Thermo Scientific. 2015. TNBSA instructions. [Online]. Available: https://www.lifetechnologies.com/order/catalog/product/28997

[90] Zhang, J., Grek, C., Ye, Z.W. et al. 2014. Pleiotropic functions of glutathione Stransferase P. Adv. Cancer Res., 122, 143-175. 2014.

[91] Kariktala, P., Mantyniemi, A., Kang, S.W. 2003. Peroxiredoxins in breast carcinoma. Clin. Cancer Res., 9, 3418-3424.

[92] Nishihira, J., Ishibashi, T., Sakai, M. et al. 1992. Characterization of cysteine residues of glutathione S-transferase P: evidence for steric hindrance of substrate 
binding by a bulky adduct to cysteine 47. Biochem. Biophys. Res. Commun., 188 (15), 424-432.

[93] Marino, F., Christobal, A., Binai, N.A. et al. 2014. Characterization and usage of the EASY-spray technology as part of an online 2D SCX-RP ultra-high pressure system. Analyst, 139(24), 6520-6528.

[94] Baeumlisberger, D., Kurz, C., Arrey, T.N. et al. 2015. Enhancing Sequence Coverage in Proteomics Studies by Using a Combination of Proteolytic Enzymes. Poster - Thermo Fischer Scientific.

[95] Chalmers, M.J., Busby, S.A., Pascal, B.D. 2011. Differential hydrogen/deuterium exchange mass spectrometry analysis of protein-ligand interactions. Expert Rev. Proteomics, 8(1), 43-59.

[96] Rob, T., Gill, P.K., Golemi-Kotra, D. 2013. An electrospray ms-coupled microfluidic device for sub-second hydrogen/deuterium exchange pulse-labelling reveals allosteric effects in enzyme inhibition. Lab On A Chip, 13, 2528-2532.

[97] Bio-Rad. 2015. Quick start bradford protein assay instruction manual.[Online]. Available: http://www.bio-rad.com/webroot/web/pdf/lsr/literature/4110065A.pdf.

[98] Duhamel, R.C., Whitehead, J.S. 1990. Prevention of nonspecific binding of avidin. Methods Enzymol., 184, 201-207.

[99] Singaravelu, R., Blais, D.R., McKay, C.S. et al. 2010. Activity-based protein profiling of the hepatitis $\mathrm{C}$ virus replication in Huh-7 hepatoma cells using a nondirected active site probe. Proteome Sci., 8(5), 1-15.

[100] Sherratt, A.R., Nasheri, N., McKay, C.S. et al. 2014. A new chemical probe for phosphatidylinositol kinase activity. Eur. J. Chem. Biol., 15(9), 1253-1256.

[101] Speers, A.E., Cravatt, B.F. 2009. Activity-Based Protein Profiling (ABPP) and Click Chemistry (CC)-ABPP by MudPIT Mass Spectrometry. Curr. Protoc. Chem. Biol., 1, 29-41. 